\title{
Regulation and Function of the Cancer Stem Cell Transcription Factors GLI1 and Kruppel-like Factor 4
}

\author{
Daniel B. Vanderbilt
}

Follow this and additional works at: https://researchrepository.wvu.edu/etd

\section{Recommended Citation}

Vanderbilt, Daniel B., "Regulation and Function of the Cancer Stem Cell Transcription Factors GLI1 and Kruppel-like Factor 4" (2015). Graduate Theses, Dissertations, and Problem Reports. 6863.

https://researchrepository.wvu.edu/etd/6863

This Dissertation is protected by copyright and/or related rights. It has been brought to you by the The Research Repository @ WVU with permission from the rights-holder(s). You are free to use this Dissertation in any way that is permitted by the copyright and related rights legislation that applies to your use. For other uses you must obtain permission from the rights-holder(s) directly, unless additional rights are indicated by a Creative Commons license in the record and/ or on the work itself. This Dissertation has been accepted for inclusion in WVU Graduate Theses, Dissertations, and Problem Reports collection by an authorized administrator of The Research Repository @ WVU.

For more information, please contact researchrepository@mail.wvu.edu. 


\title{
Regulation and Function of the Cancer Stem Cell Transcription Factors GLI1 and Krüppel-like Factor 4
}

\author{
Daniel B. Vanderbilt
}

Dissertation submitted to the School of Medicine at West Virginia University in partial fulfillment of the requirements for the degree of

\author{
Doctor of Philosophy \\ in \\ Cancer Cell Biology
}

\author{
Elena N. Pugacheva, Ph.D., Chair \\ Erik A. Bey, Ph.D. \\ Karen H. Martin, Ph.D. \\ Mohamad A. Salkeni, Ph.D. \\ Scott A. Weed, Ph.D. \\ J. Michael Ruppert, M.D., Ph.D., Mentor \\ Cancer Cell Biology Program \\ Morgantown, West Virginia
}

2015

Key Words: Breast cancer, DDR1, GLI, Hedgehog, KLF4, metastasis, pancreatic cancer, $\mathrm{SOX} 9, \beta-\operatorname{TrCP}$

Copyright 2015 Daniel B. Vanderbilt 


\title{
ABSTRACT \\ Regulation and Function of the Cancer Stem Cell Transcription Factors GLI1 and Krüppel-like Factor 4
}

\author{
Daniel B. Vanderbilt
}

Transcription factors are crucial to the normal and pathologic biology of cells. Aberrant genetic regulation underlies all facets of cancer, including metastasis, therapeutic resistance, and other clinically relevant manifestations. Krüppel-like factor 4 (KLF4) and GLI1, transcription factors that interact with DNA through conserved zinc finger domains, are implicated in human malignancy. The stability of GLI1 protein is a central determinant of Hedgehog pathway signaling output, and is regulated through interaction with the F-box protein $\beta-\operatorname{TrCP}$. In pancreatic ductal adenocarcinoma, GLI1 may be activated by oncogenic mechanisms. While KLF4 is not specific to any discrete signal pathway, KLF4 is well-characterized in numerous biological processes, including pluripotency and stress response. The functions of KLF4 are highly context-dependent, and the role of KLF4 in metastasis and other emergent phenotypes remains unclear. This dissertation addresses two distinct topics: Mechanisms underlying GLI1 stabilization in pancreatic cancer, and the function of KLF4 in the metastasis of breast cancer cells.

As an effector protein of the Hedgehog (Hh) signal pathway, GLI1 is implicated in pancreatic ductal adenocarcinoma (PDA) and other cancer types. Like other GLI proteins, GLI1 stability is regulated by the ubiquitin-proteasome system through the E3 ligase SKP1/CUL1/Fbox (SCF) protein $\mathrm{SCF}^{\beta-\operatorname{TrCP}}$. While Hh signaling is known to induce expression of the high mobility group box protein SOX9 in several contexts, the function of this signaling mechanism in PDA is poorly understood. In Chapter 2, we report the stabilization of GLI1 protein by SOX9 in PDA cells, finding that $\mathrm{SOX} 9$ inhibited $\mathrm{SCF}^{\beta-\operatorname{TrCP}}$-mediated protein degradation. In the presence of SOX9, the association of GLI1 and $\beta-\operatorname{TrCP}$ was reduced. The discovery that SOX9 interacts with the F-box domain of $\beta$-TrCP led us to question whether SOX9 could inhibit $\mathrm{SCF}^{\beta-}$ ${ }_{\mathrm{TrCP}}$ complex assembly, as this region is known to function in binding the SKP1 adaptor protein. Indeed, we observed enhanced association between SKP1 and $\beta$-TrCP upon suppression of SOX9, whereas overexpression of SOX9 led to drastically reduced SKP1- $\beta-\operatorname{TrCP}$ interaction. Additionally, SOX9 functioned to promote the nuclear tethering and degradation of $\beta-\operatorname{TrCP}$. In PDA cells, deficiency of SOX9 resulted in loss of malignant properties and cancer stem cell (CSC) traits, effects that could be rescued by suppression of $\beta$-TrCP. We also provide evidence that additional substrates of $\beta$-TrCP may be similarly regulated by SOX9. These results identify a positive feedback mechanism of GLI1 stability in PDA cells, revealing that SOX9 can inhibit $\mathrm{SCF}^{\beta-\operatorname{TrCP}}$ activity to suppress degradation of oncoproteins.

While KLF4 inhibits cell proliferation and is downregulated in some tumor types, KLF4 promotes survival and therapeutic resistance in breast cancer cells. Metastasis remains a 
significant clinical problem, yet a comprehensive understanding of the metastatic process remains elusive. In Chapter 3, we address the function of KLF4 in a mouse model of breast cancer metastasis. In triple-negative breast cancer cell lines, stable suppression of KLF4 resulted in enhanced spontaneous metastasis to the lungs and liver. Similarly, we observed fewer metastases in association with KLF4 overexpression. KLF4 had minimal impact on primary tumor initiation and growth. Although increased circulating tumor cells (CTCs) arose from KLF4-knockdown tumors, this effect was not predicted by function of KLF4 in 2D assessments of motility and invasion. Discoidin domain receptor 1 (DDR1) is a collagen-binding receptor tyrosine kinase associated with metastasis. We found that KLF4 suppresses expression of DDR1. Accordingly, suppression of KLF4 resulted in greater expression of DDR1 mRNA and protein, and increased adhesion to collagen. We propose that KLF4 inhibits metastasis of breast cancer cells through downregulation of DDR1. 
For Audrey 


\section{ACKNOWLEDGEMENTS}

Throughout my work in preparing this doctoral dissertation, I am reminded of the many individuals who have helped me along the way. To family, friends, faculty, mentors, advisors, teachers, colleagues, and so many others, I am profoundly grateful.

First, I thank my mentor and doctoral advisor, Dr. J. Michael Ruppert, for training me, and providing guidance and scientific insight throughout my years of predoctoral research. In gratitude I acknowledge other current and former lab members, including Mark Farrugia, Dr. Wentao Deng, Dr. Chen-Chung Lin, Michael Mckinstry, Jackie Metheny, and Sriganesh Sharma. All have provided expertise, advice, encouragement, and assistance. With greatest appreciation I thank Dr. Deng for his extraordinary efforts in our collaboration as co-authors. It has been a tremendous privilege to work with Dr. Deng. I also thank fellow students Mark Farrugia and Sriganesh Sharma for both research assistance and camaraderie, and offer congratulations for their recent success as doctoral candidates.

Throughout my research, many faculty and staff have assisted in my projects, and I thank Drs. Amanda Ammer and Kathleen Brundage, Emily Ellis, Sarah McLaughlin, and Brandi Underwood for crucial instruction, training, and technical assistance. Many students have also provided valuable advice and support. These include those in the Cancer Cell Biology program, in other fields, and my fellow MD-PhD students. In particular, I thank Bridget Hindman, Colton Koontz, Audrey Jajosky, and Amanda Suchanek, who have all made significant contributions to my work.

As a doctoral candidate, I have been aided, challenged, and encouraged by committee members Drs. Erik Bey, Karen Martin, Elena Pugacheva, Mohamad Salkeni, Scott Weed, and Robert Wysolmerski. I am especially grateful to Drs. Pugacheva and Weed, who took on additional responsibilities on short notice for my benefit. As Director of the Graduate Program in Cancer Cell Biology, Dr. Weed continues to work on behalf of myself and other students, and I am most thankful for his service.

Forever I will be indebted to the many scientists who have served as teachers, mentors, and advisors. I offer my most sincere appreciation to my first mentor, Dr. Allison Calhoun, who has inspired many students to pursue science at the graduate level and beyond. At WVU, I especially thank Drs. Alexey Ivanov, Lisa Salati, and Robert Wysolmerski, who have directly aided my projects and provided critical advice.

Finally, I am honored to thank Assistant Vice President for Graduate Education and former MD-PhD program director Dr. Fred Minnear, and current MD-PhD program director Dr. David Siderovsky, for their leadership and student advocacy. Without their efforts, my success and that of other students would not be possible. 


\section{TABLE OF CONTENTS}

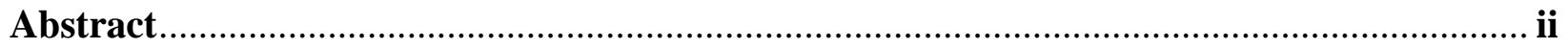

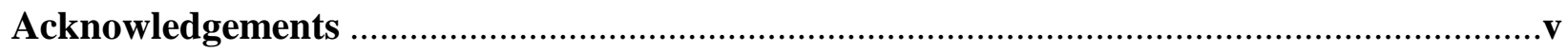

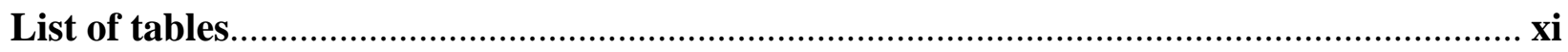

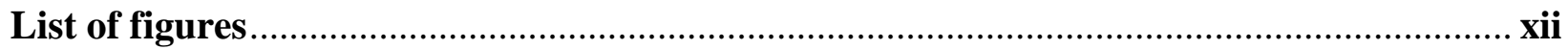

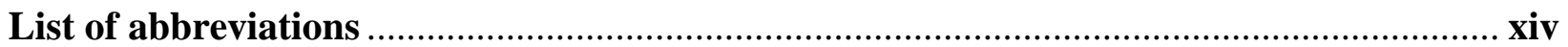

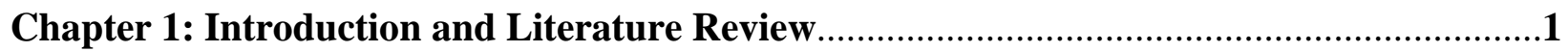

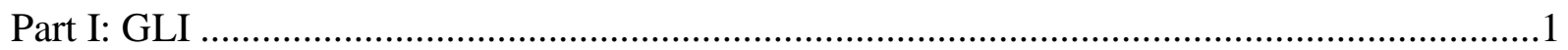

Part I overview: Hedgehog signaling, GLI proteins, and the ubiquitin-proteasome system........1

Hedgehog signal pathway .................................................................................................2

GLI family proteins as effectors of Hh signaling...................................................................

GLI discovery and structure ...............................................................................................

Genomic GLI1 and splice variants .....................................................................................

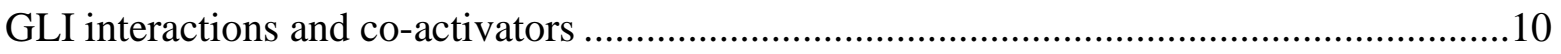

Processing of Ci/GLI proteins ............................................................................................12

Genes regulated by GLI ..................................................................................................13

Hedgehog signal pathway in cancer ...................................................................................14

Basal cell carcinoma and medulloblastoma : Archetypal Hh-driven malignancies ................15

Hedgehog signaling in pancreatic ductal adenocarcinoma .......................................................16

RAS and MAPK signaling: an alternate mechanism of GLI activation in cancer ..................20

Hedgehog signaling and GLI proteins in cancer stem cells .................................................21

Cancer stem cells .................................................................................................................22

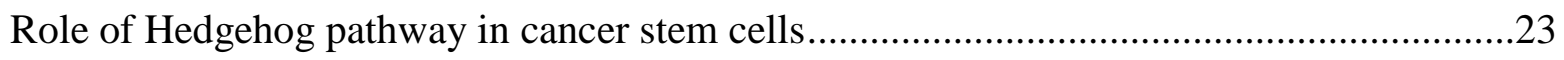

Therapeutic targeting of the Hedgehog pathway in cancer .....................................................25

Methods of Hedgehog targeting ..............................................................................................26

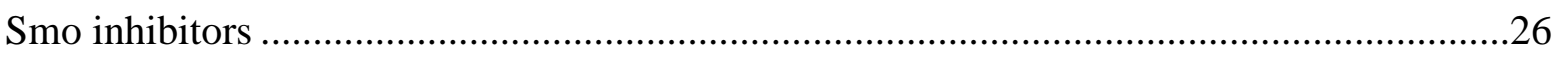

Smo inhibitors for treatment of basal cell carcinoma........................................................2 
Smo inhibitors for treatment of medulloblastoma............................................................29

Inhibitors of GLI activity .................................................................................................30

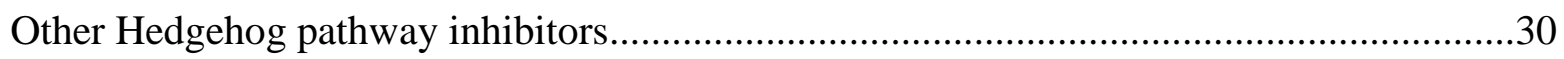

Limitations of existing Hedgehog therapies.....................................................................

The ubiquitin pathway and regulation of GLI1 protein stability................................................32

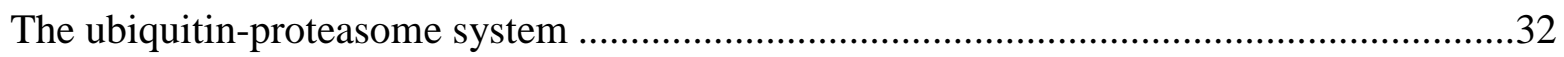

The E3 ubiquitin ligase.......................................................................................................35

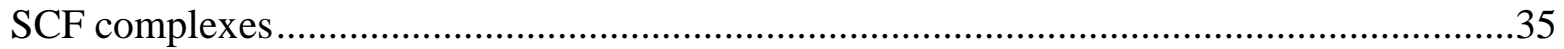

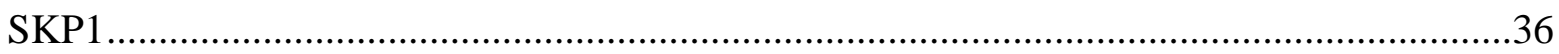

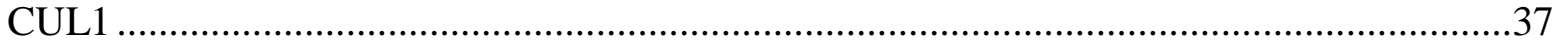

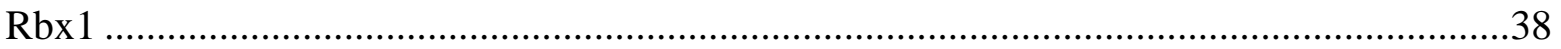

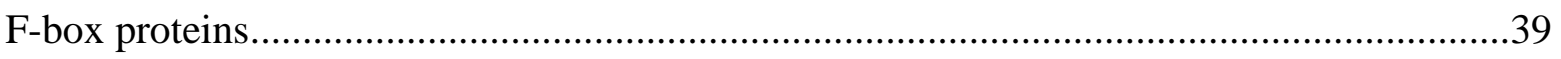

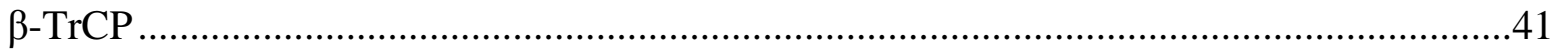

Substrates of $\beta$-TrCP and function of $\beta$-TrCP as oncoprotein or tumor suppressor ..............44

Regulation of GLI proteins and $\beta$-TrCP interactions .............................................................47

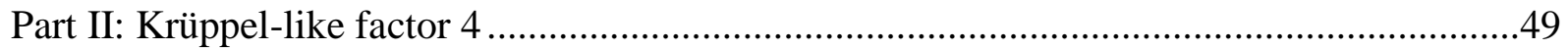

Part II overview: KLF4 and breast cancer metastasis ...........................................................49

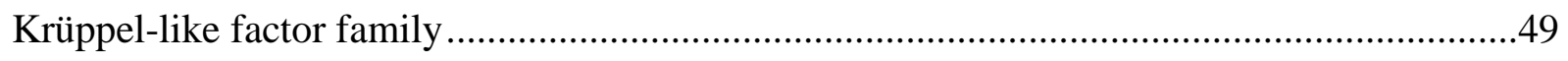

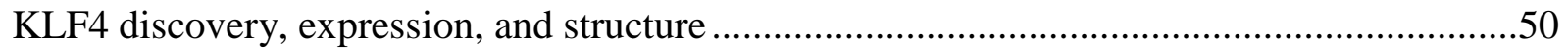

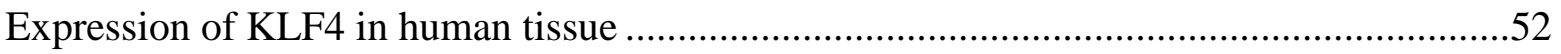

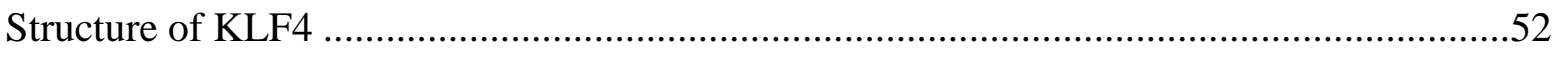

Transcriptional mechanisms of KLF4 ...............................................................................53

KLF4 as a transcriptional activator ................................................................................5

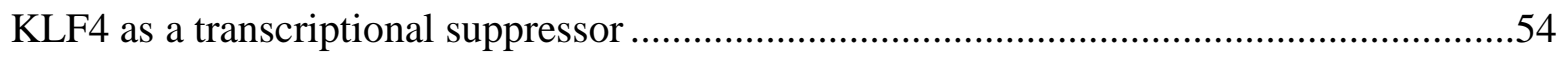

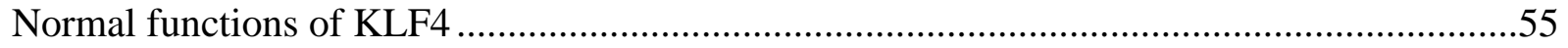

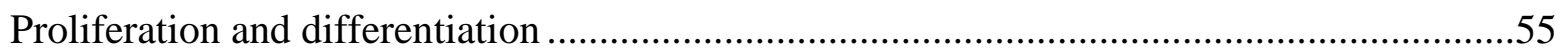

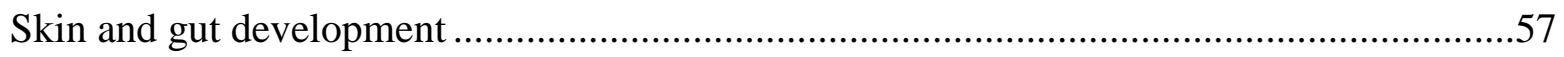

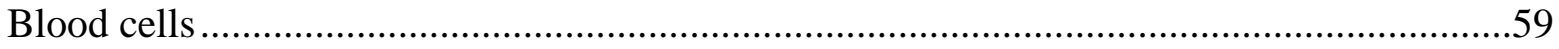

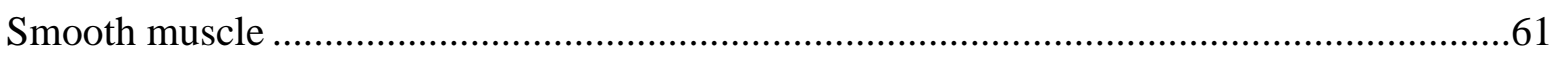

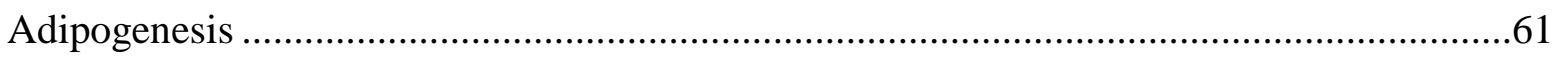

Neural development and regeneration.................................................................................62 


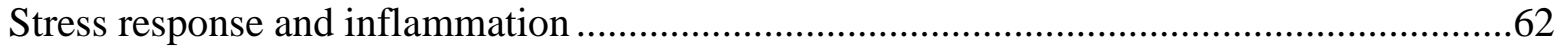

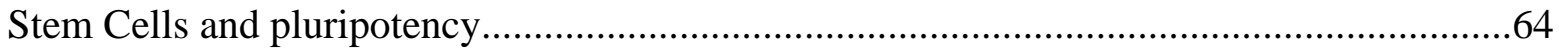

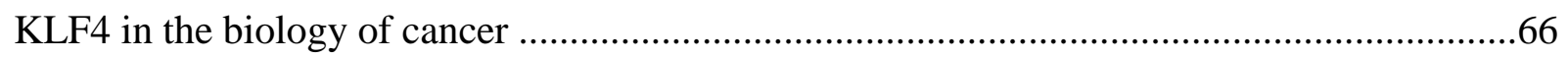

Colorectal cancer and tumor suppressive functions of KLF4 .......................................66

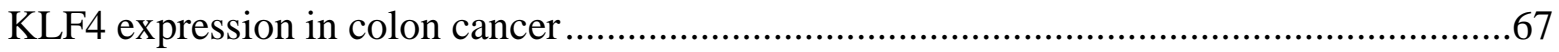

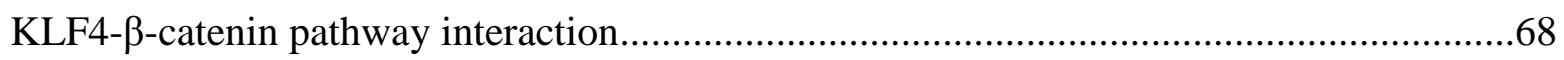

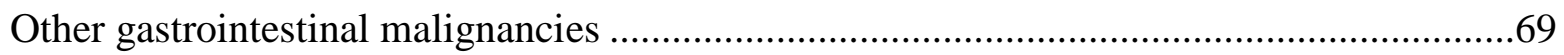

Additional tumor-suppressive functions of KLF4 ......................................................70

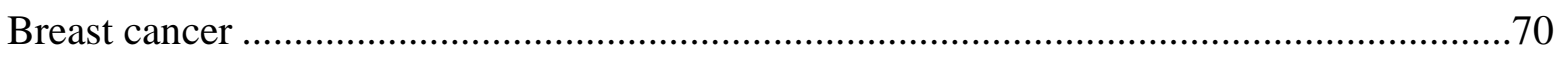

Expression of KLF4 in breast cancer ..................................................................... 71

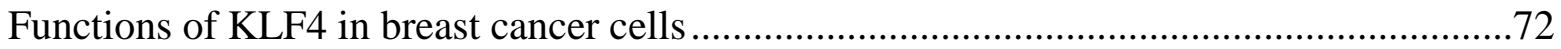

KLF4-dependent phenotypes in breast cancer cells ...................................................72

KLF4 promotes survival and drug resistance in breast cancer cells ................................73

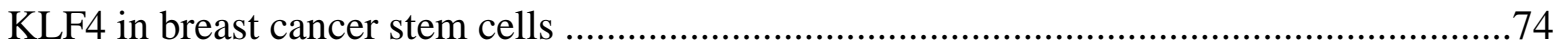

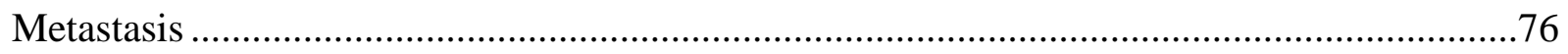

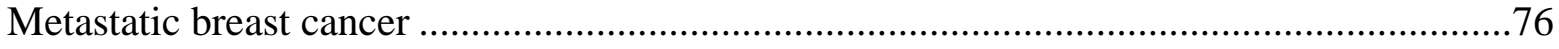

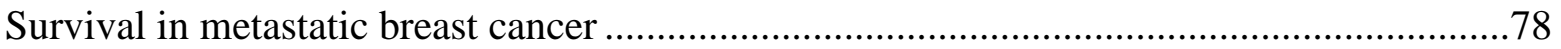

Model systems for triple-negative and claudin-low breast cancer ...................................78

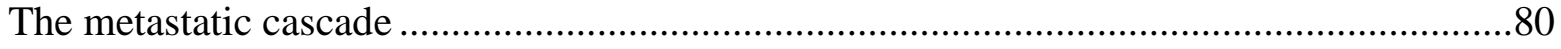

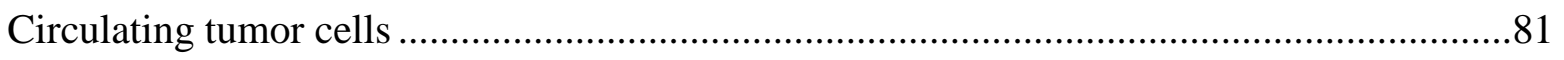

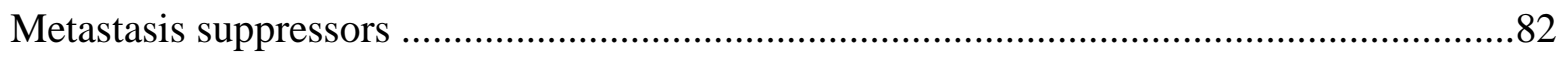

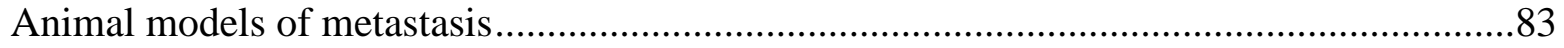

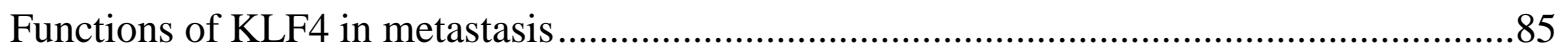

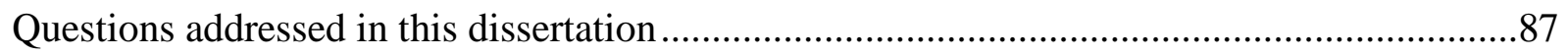

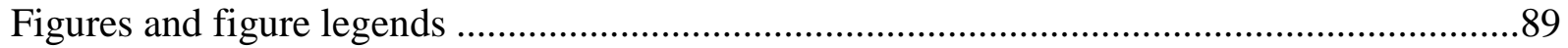

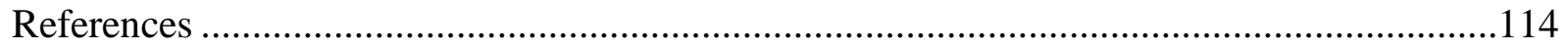

Chapter 2: SOX9 inhibits $\beta$-TrCP-mediated protein degradation to promote nuclear GLI1

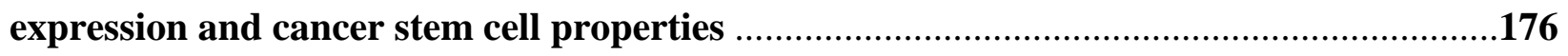

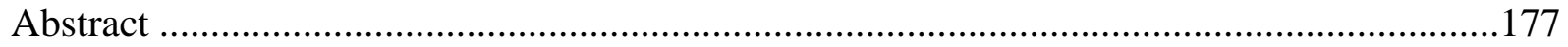

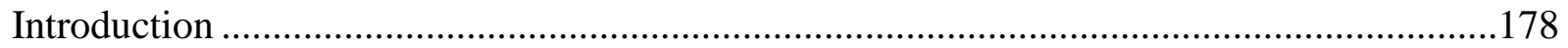




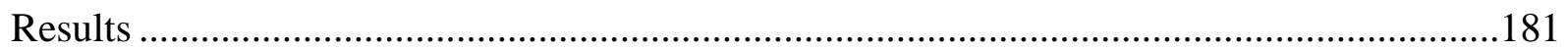

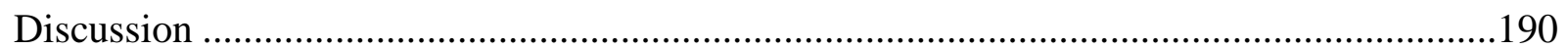

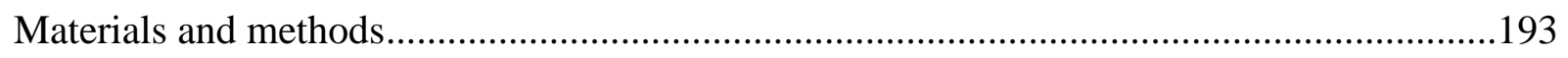

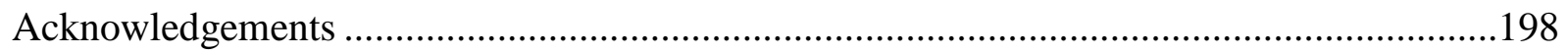

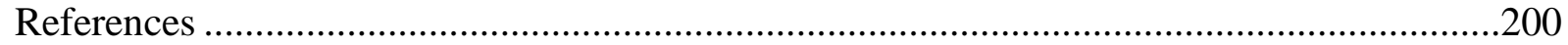

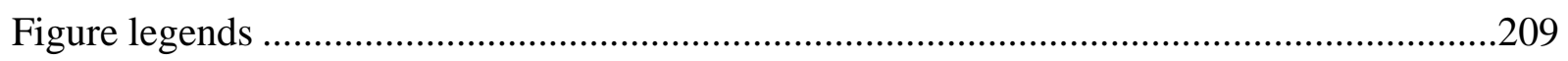

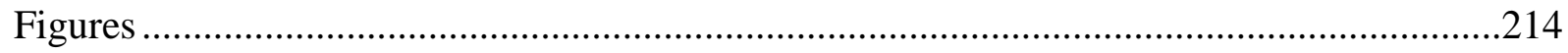

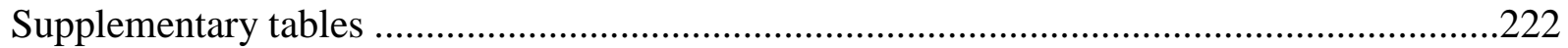

Supplementary figures and figure legends .....................................................................22

\section{Chapter 3: KLF4 Suppresses Expression of the Receptor Tyrosine Kinase DDR1 in Breast}

Cancer Cells and Inhibits Spontaneous Metastasis ..........................................................233

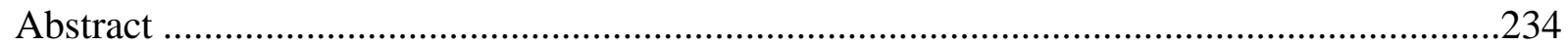

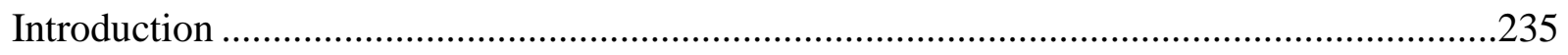

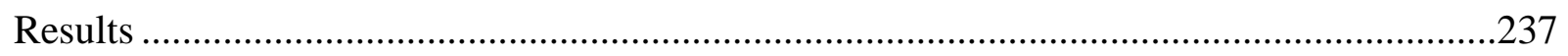

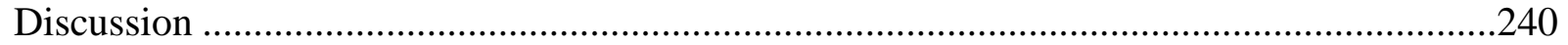

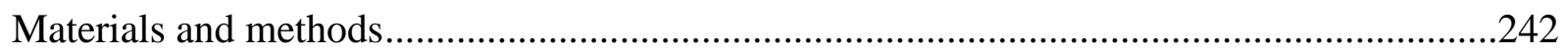

Acknowledgements ……………………………………...............................................245

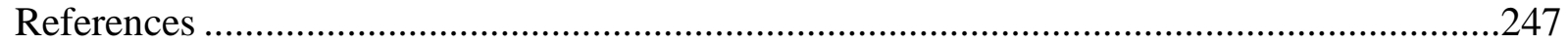

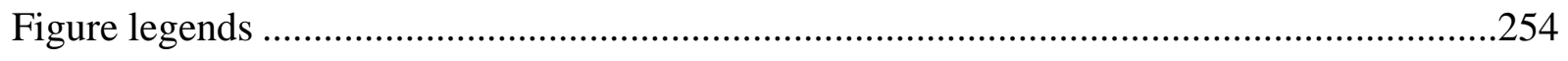

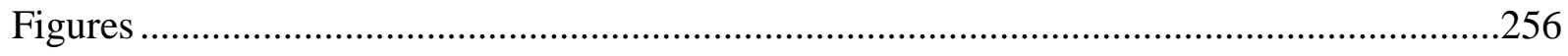

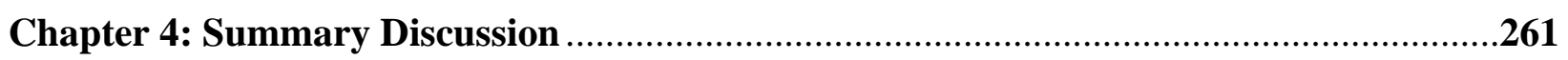

SOX9 inhibits $\beta$-TrCP-mediated degradation of GLI1 …....................................................261

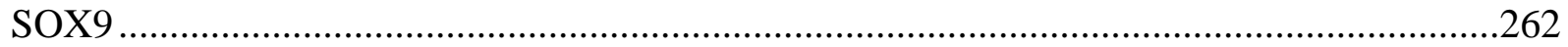

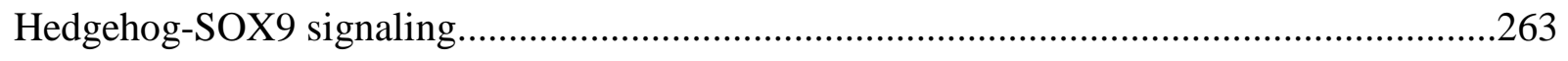

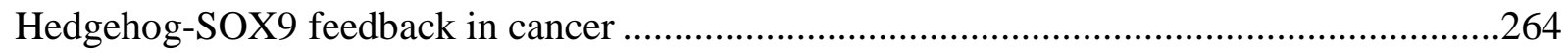

Hedgehog-SOX9 feedback in development ....................................................................266

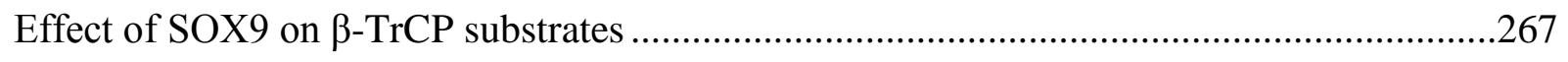

$\beta$-TrCP in cancer: Substrates and context-dependence ........................................................268

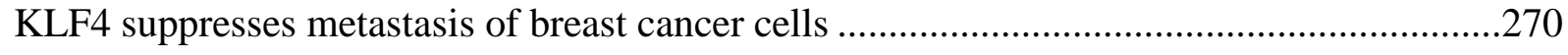




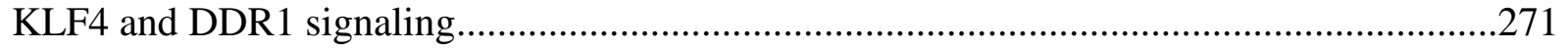

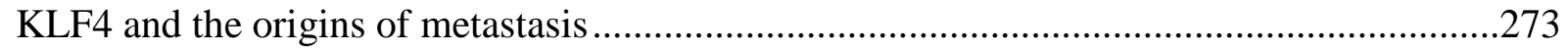

Circulating tumor cell detection and relationship to metastasis ......................................275

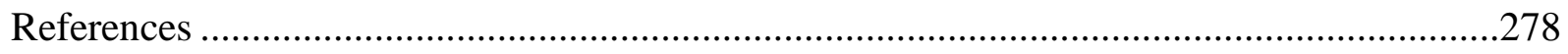




\section{LIST OF TABLES}

\section{CHAPTER 2}

Table S1 Microarray expression analyses of Sox 9 and $\beta$-TRCP in primary pancreatic cancer Table S2 Oligonucleotide sequences for siRNA and shRNA

Table S3 Oligonucleotides for real-time quantitative PCR analysis 


\section{LIST OF FIGURES}

\section{CHAPTER 1}

Figure 1 Hedgehog signaling controls mouse development through Shh, Ihh, and Dhh

Figure 2 GLI proteins are effectors of Hedgehog signaling

Figure 3 A highly simplified schematic of Hedgehog signaling in vertebrates

Figure 4 Smoothened-independence of Hedgehog signaling in Kras-driven pancreatic ductal adenocarcioma

Figure 5 Role of GLI transcriptional activity in Kras-induced pancreatic ductal adenocarcinoma

Figure 6 Hedgehog signaling in pancreatic ductal adenocarcinoma

Figure 7 Therapeutic benefit of the Hedgehog pathway inhibitor vismodegib in a randomized, double-blind, placebo-controlled trial in patients with nevoid basal cell carcinoma syndrome

Figure 8 The ubiquitin system

Figure 9 Structure of F-box containing E3 ubiquitin ligases

Figure 10 Overview of KLF structure

Figure 11 Human KLF4 locus, mRNA, and protein structure

Figure 12 KLF4 is required for the skin barrier function

Figure 13 Goblet cell deficiency in $\mathrm{Klf}^{-/-}$mice

Figure 14 KLF4 expression in the normal human colonic mucosa

Figure 15 Association of KLF4 expression with disease-free survival in colon cancer

Figure 16 Tissue-specific loss of KLF4 results in precancerous changes of the gastric epithelium

Figure 17 KLF4 promotes malignant properties in the absence of p21

Figure 18 Survival in metastatic breast cancer

Figure 19 Steps and obstacles in metastasis

Figure 20 Circulating tumor cells in the metastatic cascade

Figure 21 Spontaneous metastasis of MDA-MB-231 cells

Figure 22 Expression of miR-7 and KLF4 in brain metastases of human breast cancer.

\section{CHAPTER 2}

Figure 1 SOX9 stabilizes GLI1 in the rat RK3E epithelial model and in human PDA cells

Figure 2 Sox9 and GLI1 interact with distinct regions of $\beta$-TrCP in a mutually incompatible fashion

Figure 3 SOX9 disrupts the $\beta$-TrCP/SKP1 association and destabilizes $\beta$-TrCP in PDA cells 
Figure 4 SOX9 tethers $\beta$-TrCP within the nuclei of PDA cells and protects nuclear GLI1 from degradation

Figure 5 In a GLI1-dependent fashion, $\beta$-TrCP suppression rescues malignant properties in SOX9-deficient PDA cells

Figure $6 \quad \beta$-TrCP suppression rescues CSC properties in SOX9-deficient PDA cells

Figure 7 SOX9 stabilizes $\beta$-catenin in Panc-1 cells

Figure S1 Sox9 is activated by GLI1 in the rat RK3E epithelial model and is required for GLI1mediated malignant transformation

Figure S2 SOX9 interrupts $\beta$-TrCP-mediated GLI1 degradation

Figure S3 SOX9 is critical for cancer cell malignant properties, cancer stem cell properties and expression of $\beta$-catenin in PDA cells

Figure S4 Gene expression profile analysis of human PDA

\section{CHAPTER 3}

Figure 1 Suppression of KLF4 enhances spontaneous metastasis of MDA-MB-231 cells to the lungs and liver

Figure 2 Suppression of KLF4 enhances spontaneous metastasis of M6 cells to the lungs

Figure 3 Overexpression of KLF4 suppresses spontaneous metastasis of MDA-MB-231 and MDA-MB-435 cells

Figure 4 Effect of KLF4 expression on CTC levels, migration, and invasion of MDA-MB-231 cells

Figure 5 KLF4 suppresses DDR1 expression 


\section{LIST OF ABBREVIATIONS}

\begin{tabular}{|c|c|}
\hline 4E-BPs & 4E-binding proteins \\
\hline 4-OHT & 4-hydroxytamoxifen \\
\hline $\mathrm{ABC}$ & ATP-binding cassette \\
\hline ACTB & Actin, beta \\
\hline Ago & Argonaute \\
\hline $\mathrm{AKT}$ & Ak thymoma (Protein kinase B) \\
\hline $\mathrm{ALDH}$ & Aldehyde dehydrogenase \\
\hline ALL & Acute lymphocytic leukemia \\
\hline ALPI & Intestinal alkaline phosphatase \\
\hline $\mathrm{AMH}$ & Anti-Müllerian hormone \\
\hline AML & Acute myelogenous leukemia \\
\hline ANGPT & Angiopoetin \\
\hline AP-1 & Activator protein-1 \\
\hline APC & Adenomatous polyposis coli \\
\hline ATO & Arsenic trioxide \\
\hline ATP & Adenosine triphosphate \\
\hline AUG & Translation start codon \\
\hline Bax & BCL2-associated X protein \\
\hline BCL & B-cell lymphoma \\
\hline BMP & Bone morphogenic protein \\
\hline BRAF & B-rapidly accelerated fibrosarcoma \\
\hline BrdU & Bromodeoxyuridine \\
\hline BTB & BR-C, ttk, and bab \\
\hline $\mathrm{C}$ & Cystenine \\
\hline $\mathrm{C} / \mathrm{EBP}$ & CCAAT-enhancer-binding proteins \\
\hline cAMP & Cyclic adenosine monophosphate \\
\hline CAND1 & Cullin-associated NEDD8-dissociated protein 1 \\
\hline CBP & CREB-binding protein \\
\hline $\mathrm{c}-\mathrm{Cbl}$ & C Casitas B-lineage lymphoma \\
\hline CCR4 & Chemokine (C-C motif) receptor 4 \\
\hline CD & Cluster of differentiation \\
\hline CD11d & Integrin, alpha D \\
\hline Cdc & Cell-division cycle \\
\hline CDK & Cyclin-dependent kinases \\
\hline cDNA & Complementary DNA \\
\hline $\mathrm{CE}$ & Cornified envelope \\
\hline CENPE & Centromere protein E, 312kDa \\
\hline cGMP & Cyclic guanosine monophosphate \\
\hline ChIP & Chromatin immunoprecipitation \\
\hline
\end{tabular}




$\begin{array}{ll}\text { Ci } & \text { Cubitus interruptus } \\ \text { Cip1 } & \text { Cyclin-dependent kinase inhibitor 1 } \\ \text { Ci } & \text { Cubitus interruptus repressor form } \\ \text { CK } & \text { Cytokeratin; Casein kinase } \\ \text { CK1 } & \text { Casein kinase 1 } \\ \text { CLL } & \text { Chronic lymphocytic leukemia } \\ \text { CL } \beta \text { L } & \text { Clasto-lactacystin } \beta \text {-lactone } \\ \text { CNS } & \text { Central nervous system } \\ \text { Co-IP } & \text { Co-immunoprecipitation } \\ \text { Cos2 } & \text { Costal-2 } \\ \text { CRE } & \text { cAMP response element } \\ \text { Cre } & \text { Causes recombination } \\ \text { CREB } & \text { cAMP response element binding } \\ \text { CSC } & \text { Cancer stem cell } \\ \text { CtBP } & \text { C-terminal binding protein } \\ \text { CTC } & \text { Circulating tumor cell } \\ \text { CUL } & \text { Cullin } \\ \text { Cx43 } & \text { Connexin 43 } \\ \text { CXCR4 } & \text { C-X-C chemokine receptor type 4 } \\ \text { CYP1A1 } & \text { Cytochrome P450, family 1, subfamily A, polypeptide 1 } \\ \text { DCIS } & \text { Ductal carcinoma in situ } \\ \text { DDR } & \text { Discoidin domain receptor } \\ \text { Dhh } & \text { Desert hedgehog } \\ \text { DIM } & \text { Bis(3'-indolyl)methane } \\ \text { DNA } & \text { Deoxyribonucleic acid } \\ \text { Dox } & \text { Doxycycline } \\ \text { DPC } & \text { Days post coitum } \\ \text { Dyrk1 } & \text { Dual specificity tyrosine phosphorylation-regulated kinase 1 } \\ \text { E } & \text { Glutamic acid } \\ \text { E2F1 } & \text { E2F transcription factor 1 } \\ \text { E6AP } & \text { Ubiquitin-protein ligase E3A } \\ \text { ECM } & \text { Extracellular matrix } \\ \text { EDC3 } & \text { Enhancer of mRNA decapping } 3 \text { homolog } \\ \text { EGR } & \text { Early growth response protein 1 } \\ \text { eIF } & \text { Eukaryotic initiation factor } \\ \text { EKLF } & \text { Erythroid Krüppel-like factor } \\ \text { EMCV } & \text { Encephalomyocarditis virus } \\ \text { EMI } & \text { Early mitotic inhibitor } \\ \text { EMT } & \text { Epithelial-mesenchymal transition } \\ \text { ER } & \text { Estrogen receptor } \\ & \end{array}$




\begin{tabular}{|c|c|}
\hline ES & Embryonic stem \\
\hline Esrrb & Estrogen-related receptor beta \\
\hline EZF & Epithelial zinc finger \\
\hline $\mathrm{F}$ & Phenylalanine \\
\hline FAP & Familial adenomatous polyposis \\
\hline FBX & F-box \\
\hline FDA & US Food and Drug Administration \\
\hline FOX & Forkhead box \\
\hline Foxa2 & Forkhead box A2 \\
\hline $\mathrm{Fu}$ & Fused \\
\hline FXR1 & Fragile X-related protein 1 \\
\hline G & Glycine \\
\hline GAL4 & Galactose-responsive transcription factor 4 \\
\hline GANT & GLI antagonist \\
\hline GBR & GLI binding region \\
\hline GEM & Genetically engineered mouse \\
\hline GFP & Green fluorescent protein \\
\hline GI & Gastrointestinal \\
\hline GKLF & Gut-enriched Krüppel-like factor \\
\hline $\mathrm{GLI}^{\mathrm{A}}$ & GLI activator form \\
\hline $\mathrm{GLI}^{\mathrm{R}}$ & GLI repressor form \\
\hline GPCR & G protein-coupled receptor \\
\hline GPS & Global protein stability \\
\hline GSK3 & Glycogen synthase kinase 3 \\
\hline GTP & Guanosine-5'-triphosphate \\
\hline GTPase & GTP hydrolase \\
\hline GW182 & Trinucleotide repeat containing 6A \\
\hline $\mathrm{H}$ & Histidine \\
\hline HAT & Histone acetyltransferase \\
\hline $\mathrm{HCV}$ & Hepatitis C virus \\
\hline HDAC & Histone deacetylase \\
\hline HDC & Histidine decarboxylase \\
\hline HECT & Homologous to the E6-AP carboxyl terminus \\
\hline HER2 & Human epidermal growth factor receptor 2 \\
\hline Hh & Hedgehog \\
\hline HHARI & Human homolog of Ariadne \\
\hline HHAT & Hedgehog acetyl transferase \\
\hline Hib & Hh-induced MATH and BTB domain-containing protein \\
\hline HMEC & Human mammary epithelial cells \\
\hline HMG & High mobility group \\
\hline
\end{tabular}




\begin{tabular}{|c|c|}
\hline hMSC & Human mesenchymal stem cell \\
\hline hnRNP-U & Heterogeneous nuclear ribonucleoprotein U \\
\hline HoxD & Homeobox protein D \\
\hline HPDE & Human pancreatic ductal epithelial \\
\hline HUVEC & Human umbilical vein endothelial cell \\
\hline IBMX & 3-isobutyl-1-methylxanthine \\
\hline IFITM3 & Interferon-induced transmembrane protein 3 \\
\hline IFN & Interferon \\
\hline IGF & Insulin-like growth factor \\
\hline IGFBP & Insulin-like growth factor-binding protein \\
\hline Ihh & Indian hedgehog \\
\hline IL & Interleukin \\
\hline iNOS & Inducible nitric oxide synthase \\
\hline iPS & Inducible pluripotent stem \\
\hline IRES & Internal ribosome entry sites \\
\hline ITC & Isolated tumor cell \\
\hline ІкВ & Inhibitor of kappa B \\
\hline $\mathrm{K}$ & Lysine \\
\hline KAT5 & Lysine acetyltransferase 5 \\
\hline $\mathrm{kDa}$ & kiloDalton \\
\hline Kif7 & Kinesin family member 7 \\
\hline Kip1 & Cyclin-dependent kinase inhibitor 1B \\
\hline KLF & Krüppel-like factor \\
\hline Kras & Kirsten rat sarcoma \\
\hline Lefty 1 & Left-right determination factor 1 \\
\hline LIF & Leukemia inhibitory factor \\
\hline $\mathrm{LOH}$ & Loss of heterozygosity \\
\hline LPS & Lipopolysaccharide \\
\hline LSL & Lox stop lox \\
\hline $\mathrm{m}^{7} \mathrm{G}$ & 7-methylguanylate \\
\hline MAD & Mothers agains decapentaplegic \\
\hline MAPK & Mitogen-activated protein kinase \\
\hline MATH & Meprin and TRAF homology \\
\hline MCL & Myeloid cell leukemia 1 \\
\hline MDM2 & Mouse double minute 2 homolog \\
\hline MEF & Mouse embryonic fibroblasts \\
\hline Meis & Meis homeobox \\
\hline MEK & Mitogen/extracellular signal-regulated kinase \\
\hline MET & Mesenchymal-epithelial transition \\
\hline Met30 & Methionine requiring 30 \\
\hline
\end{tabular}




$\begin{array}{ll}\text { MH2 } & \text { Mad homology 2 } \\ \text { Min } & \text { Multiple intestinal neoplasia } \\ \text { miR } & \text { microRNA } \\ \text { miRNP } & \text { miR-protein complex } \\ \text { MMLV } & \text { Moloney murine leukemia virus } \\ \text { MMP } & \text { Matrix metalloprotein } \\ \text { MMS } & \text { Methyl methanesulfonate } \\ \text { MMTV } & \text { Mouse mammary tumor virus } \\ \text { mRNA } & \text { Messenger RNA } \\ \text { mRNP } & \text { mRNA-protein complex } \\ \text { MUC-1-C } & \text { Mucin 1, cell surface associated } \\ \text { MUC2 } & \text { Mucin 2, oligomeric mucus/gel-forming } \\ \text { Nanog } & \text { Nanog homeobox } \\ \text { NBCCS } & \text { Nevoid basal cell carcinoma syndrome } \\ \text { NEDD } & \text { Neural precursor cell expressed developmentally down-regulated protein } \\ \text { Nes } & \text { Nestin } \\ \text { NF-kB } & \text { Nuclear factor kappa-light-chain-enhancer of activated B cells } \\ \text { NK } & \text { Natural killer } \\ \text { NLS } & \text { Nuclear localization signal; nuclear localization sequence } \\ \text { NOD/SCID/ } \gamma & \text { Non-obese diabetic severe combined immunodeficiency gamma } \\ \text { NRAS } & \text { Neuroblastoma RAS } \\ \text { NSG } & \text { NOD/SCID/ } \gamma \\ \text { Oct4 } & \text { Octamer-binding transcription factor 4 } \\ \text { P } & \text { Proline } \\ \text { PABP } & \text { Poly (A)-binding protein } \\ \text { PanIN } & \text { Pancreatic intraepithelial neoplasia } \\ \text { Pax } & \text { Paired box } \\ \text { Pbx } & \text { Pre-B-cell leukemia transcription factor } \\ \text { PCR } & \text { Polymerase chain reaction } \\ \text { PDA } & \text { Pancreatic ductal adenocarcinoma } \\ \text { PDCD4 } & \text { Programmed cell death protein 4 } \\ \text { PDD } & \text { Processing determinant domain } \\ \text { PDGFR } \beta & \text { Platelet-derived growth factor receptor beta } \\ \text { PDX } & \text { Pancreatic and ductal homeobox 1; Patient-derived xenograft } \\ \text { PEST } & \text { Proline glutamine serine threonine } \\ \text { PFKP } & \text { Platelet isoform of phosphofructokinase } \\ \text { Pft1a } & \text { Pancreas-specific transcription factor 1a } \\ \text { PHD } & \text { Plant homeodomain } \\ \text { PI3K } & \text { Phosphoinositide 3-kinase } \\ \text { Pias1 } & \text { Protein inhibitor of activated STAT 1 } \\ \text { PKA } & \text { Protein kinase A } \\ & \\ & \end{array}$




$\begin{array}{ll}\text { PKC } & \text { Protein kinase C } \\ \text { POZ } & \text { Pox virus and zinc finger } \\ \text { PPAR } \gamma & \text { Peroxisome proliferator-activated receptor gamma } \\ \text { PR } & \text { Progesterone receptor } \\ \text { PTB } & \text { Phosphotyrosine-binding } \\ \text { Ptch } & \text { Patched } \\ \text { RAS } & \text { Rat sarcoma } \\ \text { RASA1 } & \text { RAS p21 protein activator (GTPase activating protein) 1 } \\ \text { Rb } & \text { Retinoblastoma } \\ \text { Rbx1 } & \text { Ring-box 1 } \\ \text { Rdx } & \text { Roadkill } \\ \text { RelA } & \text { V-rel avian reticuloendotheliosis viral oncogene homolog A } \\ \text { REN/KCTD11 } & \text { Potassium channel tetramerization domain-containing 11 } \\ \text { RGC } & \text { Retinal ganglion cell } \\ \text { RING } & \text { Really interesting new gene } \\ \text { RNA } & \text { Ribonucleic acid } \\ \text { S/T } & \text { Serine/threonine } \\ \text { SAF-1 } & \text { Serum amyloid A activating factor 1 } \\ \text { SAP18 } & \text { Sin3A-associated protein 18 } \\ \text { SCC } & \text { Squamous cell carcinoma } \\ \text { SCF } & \text { SKP2/CUL1/F-box } \\ \text { Scon2 } & \text { Sucrose consumption 2 } \\ \text { SEB } & \text { Surgically-eligible basal cell carcinoma } \\ \text { SH } & \text { Src homology } \\ \text { Shh } & \text { Sonic hedgehog } \\ \text { shRNA } & \text { small hairpin RNA } \\ \text { siRNA } & \text { small interfering RNA } \\ \text { SKI } & \text { Sloan-Kettering Institute } \\ \text { SKP } & \text { S-phase kinase-associated protein } \\ \text { Slmb } & \text { Supernumerary limbs } \\ \text { SM } & \text { Smooth muscle } \\ \text { SMA } & \text { Smooth muscle actin } \\ \text { Sma } & \text { Small body size } \\ \text { SMAD } & \text { Sma and MAD-related } \\ \text { SMC } & \text { Smooth muscle cell } \\ \text { Smo } & \text { Smoothened } \\ \text { SOCS-3 } & \text { Suppressor of cytokine signaling-3 } \\ \text { SOX } & \text { SRY-box } \\ \text { Sp1 } & \text { Specificity protein 1 } \\ \text { SPOP } & \text { Speckle-type POZ protein } \\ \text { SPR } & \text { Surface plasmon resonance } \\ & \\ & \end{array}$




\begin{tabular}{|c|c|}
\hline SPRED1 & Sprouty-related EVH1 domain containing-protein 1 \\
\hline SPRR & Small proline rich \\
\hline SRY & Sex-determining region $\mathrm{Y}$ \\
\hline STAT3 & Signal transducer and activator of transcription 3 \\
\hline $\mathrm{SuFu}$ & Suppressor of fused \\
\hline SUMO & Small ubiquitin-like modifier \\
\hline SWI/SNF & Switch sucrose nonfermentable \\
\hline TAF9 & RNA polymerase II, TATA box binding protein-associated factor 9 \\
\hline TAg & SV40 large T-antigen \\
\hline TBP & TATA binding protein \\
\hline Tbx & T-box \\
\hline TCF4 & T-cell factor 4 \\
\hline TDF & Testis-determining factor \\
\hline TFIID & Transcription factor IID \\
\hline TGF- $\beta$ & Transforming growth factor $\beta$ \\
\hline TNBC & Triple negative breast cancer \\
\hline TNF & Tumor necrosis factor \\
\hline TPA & 12-O-tetradecanoylphorbol-13-actetate \\
\hline TTP & Time to progression \\
\hline twhh & Tiggy-winkly hedgehog \\
\hline UBCE2L3 & Ubiquitin-conjugating enzyme E2-L3 \\
\hline UbR & Ubiquitin receptor protein \\
\hline uPAR & Urokinase receptor \\
\hline UPS & Ubiquitin proteasome system \\
\hline UVB & Ultraviolet B \\
\hline VCAM-1 & Vascular cell adhesion molecule 1 \\
\hline VEGF & Vascular endothelial growth factor \\
\hline Waf1 & Wild-type p53-activated fragment 1 \\
\hline WBC & White blood cell \\
\hline WD & Tryptophan aspartate \\
\hline WT & Wild type \\
\hline Zeb & Zinc finger E-box-binding homeobox \\
\hline$\beta$-TrCP & Beta-transducin repeat-containing protein \\
\hline
\end{tabular}




\section{CHAPTER 1}

\section{Introduction and Literature Review}

Chapter 1 is divided into two parts, with a broad focus on zinc finger proteins that function as major regulators of cell fate in development and cancer. The first section contains a brief overview of GLI proteins and the Hedgehog signaling pathway, a focused introduction to GLI and Hedgehog signaling in cancer including therapeutic applications, and a review of the

mechanisms determining the regulation of GLI protein by $\mathrm{SCF}^{\beta-\operatorname{TrCP}}$. The second component describes the normal functions of another major regulator of cell fate, Krüppel-like factor 4 (KLF4), the context-dependent roles of KLF4 in cancer, and current concepts in metastasis research as they may relate to KLF4.

\section{Part I: GLI}

\section{Part I overview: Hedgehog signaling, GLI proteins, and the ubiquitin-proteasome system}

The hedgehog $(\mathrm{Hh})$ signal pathway is critical in embryonic development and has numerous additional functions, including the maintenance of adult stem cells. GLI proteins are zinc finger transcription factors and are effectors of vertebrate Hh signaling. While the Hedgehog pathway was first characterized in Drosophila melanogaster, GLI1 was originally discovered as a candidate oncogene in human tumors by purification of amplified genomic sequences. Aberrant activation of GLI proteins, including GLI1, a potent transcriptional activator of Hhresponsive genes, contributes to numerous human malignancies. GLI protein stability and 
processing is regulated by the ubiquitin proteasome system (UPS), and the interaction of GLI with the $\mathrm{E} 3$ ubitquitin ligase $\mathrm{SCF}^{\beta-\operatorname{TrCP}}$ is a crucial determinant of Hh signaling output. For this reason, mechanisms governing GLI1 stability have significant translational relevance. This introductory section reviews some elements of Hh-GLI signaling, emphasizing the role of this pathway in certain malignancies, and describes the biochemical basis of GLI protein stability. In the Results section (Chapter 2), a novel mechanism governing GLI1 stability is presented, along with biological effects of this mechanism in the context of pancreatic cancer cells.

\section{Hedgehog signal pathway}

In 1980, the hedgehog gene $(h h)$ was identified through a screening process to identify genes that regulate the development of Drosophila larvae (1). In $h h$ mutant larvae, the cuticle was short and spiked, possibly in imaginative resemblance to a hedgehog. In vertebrates, the $H h$ family of genes crucially regulates development (Fig. 1) as well as diverse cellular processes such as differentiation, cell fate, and survival (2). The components and mechanisms functionally associated with Hh proteins make up the Hedgehog signaling pathway (Hh pathway). Much of the pathway found in Drosophila is well-conserved in vertebrates, including Hh pathway homologues found in mouse and human cells, although some structural and functional differences have been characterized $(3,4)$.

In vertebrate species, multiple $H h$ homologues are found, including three in mammals. These are Desert hedgehog (Dhh), Indian hedgehog (Ihh), and Sonic hedgehog (Shh). Additional $H h$ genes are sometimes found elsewhere in the animal kingdom, such as the curiously titled tiggy-winkle hedgehog (twhh) of the zebrafish (5). The proteins encoded by Hh genes share 
important features aside from droll allusions to video games and juvenile literature.

Phylogenetically, Dhh is more closely related to Drosophila Hh, whereas Shh and Ihh are more similar to each other. Shh is most widely expressed, and Shh deletion results in severe defects including cyclopia (6). Hh proteins undergo substantial posttranslational processing, involving an autocatalytic cleavage event and subsequent addition of cholesterol and palmitic acid moieties. Additional mechanisms release Hh peptides from the cell surface, where they may subsequently move towards distant targets, establishing a gradient. The mechanisms through which Hh proteins move between cells are less understood in comparison to intracellular Hh events (2).

The Patched (Ptch) gene encodes a transmembrane protein $(7,8)$ that functions as the receptor for Hh ligands (9). The Drosophila homologue was previously identified through genetic screening as belonging to the Hh signaling pathway, functioning downstream of Hh (10). In mammals there is an additional Ptch gene, Ptch2 $(11,12)$. Additionally, a number of proteins have been identified that act as co-receptors of Hh ligands (13). The GLI family of transcription factors, effector proteins of Hh signaling, regulates Ptch1 and Ptch2 expression such that Ptch expression is induced by Hh signaling. As Ptch protein, in the absence of Hh ligand, serves to suppress Hh signaling, GLI-mediated upregulation of Ptch serves as a negative feedback mechanism.

An additional transmembrane component of the Hh signal pathway is the protein Smoothened (Smo), a seven-transmembrane G protein-coupled receptor $(\mathrm{GPCR})(14,15)$. Smo was originally hypothesized to act as a receptor for Hh ligands, the role now attributed to Ptch. As the physiologic ligand or ligands for Smo remain unresolved, Smo is an orphan receptor (13). In the absence of Hh ligands, Ptch prevents the membrane localization of Smo, which in 
vertebrates occurs at the primary cilia (16). The binding of Ptch by Hh ligands permits the translocation of Smo from cytoplasmic vesicles to the plasma membrane at the primary cilia $(17,18)$. The mechanisms whereby Ptch regulates Smo are incompletely understood, but are thought to involve signaling by small molecules (19). Evidence suggests Ptch can regulate transport of oxysterols, which in turn activate Smo (20-22). While homozygous null Shh, Ihh, or Dhh mice develop to term, genetic ablation of Smo is lethal in embryogenesis (23).

In Drosophila, Hh signaling regulates development through the transcription factor Cubitus interruptus (Ci), of which the GLI protein family found in vertebrates is homologous $(24,25)$. This regulation occurs through a multiprotein complex involving Hh pathway mediators such as Fused ( Fu), Costal2 (Cos2), and Suppressor of Fused (SuFu). The signal transduction in vertebrates is thought to occur in a similar process, including the involvement of microtubules, although the localization of the signal transduction machinery to the primary cilia distinguishes Hh signaling in vertebrates (13). Some aspects of vertebrate Hh signal transduction may be independent of the cilia (26).

The stability and processing of $\mathrm{Ci}$, and of the GLI family in vertebrates, is determined through interaction with the UPS, and this interaction is heavily regulated by phosphorylation events involving numerous kinases (27). Kif7, homologous to the Drosophila protein Cos2, acts as a scaffold for kinases including Protein kinase A (PKA), Glycogen synthase kinase 3, (GSK3), and Casein kinase 1 (CK1)(13). Kif7, a member of the kinesin motor protein superfamily, also serves to regulate primary cilia architecture, which appears essential to proper control of Hh signaling (28). Vertebrate GLI proteins are negatively regulated by $\mathrm{SuFu}$, and $\mathrm{Hh}$ 
signaling both recruits the $\mathrm{SuFu}-\mathrm{GLI}$ complex to the cilia and promotes dissociation of $\mathrm{SuFu}$ and GLI proteins (29).

Hh signaling, as transduced through Smo and other mediators, modulates the interaction of $\mathrm{Ci}$ and GLI proteins with a Skp, Cullin, F-box (SCF) multiprotein complex. The SCF complex is an $\mathrm{E} 3$ ubiquitin ligase, acting to ubiquitylate the $\mathrm{Ci} / \mathrm{GLI}$ substrate and thereby target $\mathrm{Ci} / \mathrm{GLI}$ to the proteasome for degradation or processing. The F-box component is responsible for recognition of the Ci/GLI substrate. In Drosophila, the responsible F-box protein is Supernumerary limbs (Slimb/Slmb) (30). The homologous protein in vertebrates is F-box/WD repeat protein 1A $(\beta-\mathrm{TrCP})(31)$. $\mathrm{Ci} / \mathrm{GLI}$ proteins are known substrates of other $\mathrm{E} 3$ ubiquitin ligases in addition to $\mathrm{SCF}^{\mathrm{Slimb} / \beta-\mathrm{TrCP}}$, as is noted in the section "Processing of Ci/GLI proteins".

This dissertation is primarily focused on the stability of GLI1 protein in a context where GLI may be regulated by mechanisms other than classical Hh signaling (32-34). Classical Hh signaling is reviewed for historical context and clinical relevance. The interaction between $\beta$ TrCP and GLI1 is of great importance in both classical Hh signaling and in alternative mechanisms of GLI activation. As $\beta$-TrCP targets GLI1 for proteasomal degradation, reduced $\beta$ TrCP/GLI1 interaction is hypothesized to result in GLI1 accumulation, with consequences including oncogenic effects of GLI1 in malignancy (35).

\section{GLI family proteins as effectors of Hh signaling}

Whereas some chordates express only a single, Ci-like GLI protein (36), in vertebrates the processing-dependent transcriptional regulatory functions of $\mathrm{Ci}$ are divided amongst multiple 
GLI proteins (Fig. 2). In mammals, GLI1 and GLI2 may both function as transcriptional activators of Hh target genes, whereas transcriptional repression is accomplished by GLI2 and GLI3 (37). The greater functional overlap between GLI1 and GLI2, as compared to GLI1 and GLI3, is exemplified in mice, in which mutation of GLI2, but not GLI3, results in a severe developmental phenotype in combination with homozygous GLI1 mutations (38). The proteolytic cleavage of GLI2 and GLI3 into the repressor forms resembles the Ci processing found in Drosophila (6). This processing into cleaved forms occurs via the UPS, and is mediated by the $\mathrm{E} 3$ ligase $\mathrm{SCF}^{\mathrm{Slimb} / \beta-\mathrm{TrCP}}$ as described.

Hedgehog signaling positively and negatively regulates the transcription of Hh target genes by modulating the balance between GLI activator $\left(\mathrm{GLI}^{\mathrm{A}}\right)$ and GLI repressor $\left(\mathrm{GLI}^{\mathrm{R}}\right)$ forms, or the Ci homologue in Drosophila. GLI3 is most similar to Ci protein (2). In principle, the GLI ${ }^{\mathrm{A}}$ and GLI ${ }^{\mathrm{R}}$ forms could represent any of the GLI family, with the caveat that GLI1 does not have the N-terminal repressor domain and thus cannot function as $\mathrm{GLI}^{\mathrm{R}}$. The effect of Hh ligand binding is to tip this balance towards $\mathrm{GLI}^{\mathrm{A}}$ (37). In a simplified model, Hh signaling can be seen to effect an increase in $\mathrm{GLI}^{\mathrm{A}}$ proteins, and a decrease in $\mathrm{GLI}^{\mathrm{R}}$ forms (Fig. 3). In this manner, Hh signaling functions to transcriptionally promote $\mathrm{Hh}$ target gene expression.

A complicating factor in vertebrate $\mathrm{Hh}$ signaling is that GLI proteins directly influence GLI expression, an example of positive feedback. The GLI family of proteins can bind to promoter response elements and regulate transcription of GLI genes. Indeed, one of the most recognized transcriptional consequences of $\mathrm{Hh}$ signaling is upregulation of $G L I I$, and so $\mathrm{Hh}$ signal transduction can directly increase expression of the potent Hh effector protein, GLI1 (39). In the classical Hh signaling pathway, GLI1 seems to function mainly to reinforce pathway 
activation, and temporally extends the cellular response to Hh ligands $(2,40)$. This effect may be attributed to the positive feedback effect in which GLI1 directly promotes transcription of GLII. GLI proteins regulate each other through coordinated mechanisms that determine pathway output through the balance of GLI activator $\left(\mathrm{GLI}^{\mathrm{A}}\right)$ and GLI repressor $\left(\mathrm{GLI}^{\mathrm{R}}\right)$ functions. This process has been described as a "GLI code" (40). Deletion of GLI3 can lead to ligand independent GLI activity, supporting a model of regulation by the three mammalian GLI proteins. In the absence of Hh ligand, GLI3 directly represses transcription of Gli1 and Gli2. GLI2 protein also binds the promoters of Gli1 and Gli2 as shown by chromatin immunoprecipitation (ChIP) (41). In the canonical Hh pathway, GLI ${ }^{\mathrm{R}}$ activity is suppressed by Hh ligand, and this effect may be reproduced through GLI3 deletion. GLI2 is then able to promote expression of Gli1 and Gli2, leading to increased promotion of Gli1 transcription by GLI1 protein. Additionally, GLI1 does seem to promote expression of Gli2, although likely through indirect mechanisms (42). Genetic lesions in cancer cells may also make GLI1 independent of Hh signaling, as discussed later.

\section{GLI discovery and structure}

In 1987 , a gene found to be highly amplified in a malignant glioma was named $G L I$, and traced to chromosome 12q13 (43). The GLI gene was then cloned from a glioma cell line with 75-fold amplification of GLI (44). Within the cDNA clone, a $3.3 \mathrm{~kb}$ open reading frame predicted a peptide of 1,106 residues with a calculated mass of $118 \mathrm{kDa}$, and containing five repeats of a zinc finger motif. GLI2 and GLI3 were later discovered through low stringency hybridization with GLI cDNA (45,46); GLII is a retronym for GLI. The Drosophila gene Cubitus interruptus (ci) was cloned later, and immediately recognized to have sequence homology to human GLI (25). 
Three GLI genes and corresponding proteins are found in mammals, likely derived from duplications of an ancestral gene. Among the most obvious and conserved features of GLI proteins are the five zinc fingers, which are of the $\mathrm{C}_{2} \mathrm{H}_{2}$ type. The $\mathrm{C}_{2} \mathrm{H}_{2}$ zinc finger is a common structural motif and is composed of a $\beta$-hairpin and an $\alpha$-helix. Two cysteine residues within the "zinc knuckle" of the $\beta$-hairpin, as well as two histidine residues from the C-terminal end of the $\alpha$-helix, are responsible for the coordination of a zinc ion (47). In GLI proteins, the zinc finger domains interact with DNA. The sequence 5'-GACCACCCA-3' functions as a binding site for the GLI family of transcription factors, and has been shown to be a Shh-responsive element (48).

GLI proteins are also characterized by a C-terminal transactivation domain. This sequence is essential to the transcriptional function of Hh target genes through interaction with GLI co-activators (49). Notably, GLI1 lacks the N-terminal repressor domain that is found in GLI2 and GLI3 proteins. This absence is reflected in the role of GLI1 as the most potent transcriptional activator in the Hh signal pathway (40), and also the lesser mass of GLI; although GLI1 protein has a calculated mass of $118 \mathrm{kDa}$, it comigrates in denaturing gels with proteins of 150kDa (46). GLI1 also lacks the processing determinant domain found in GLI2 and GLI3. Lack of this domain partially explains why GLI1 is completely degraded by the proteasome (50). Other characterized features of GLI proteins include sites of phosphorylation and other posttranslational modifications, degron motifs that mediate protein stability, and SUFU binding sites (37). 


\section{Genomic GLII and splice variants}

GLI spans around $12 \mathrm{~kb}$ of genomic DNA and is located at 12q13.2-q13.3. The 5' exons 1 and $1 \mathrm{~A}$ are non-coding, and splice variants affecting the non-coding exons have been reported (51). There are 11 coding exons distributed as exons 2-12 (52). Several variant forms of GLII are described. Skipping of exons 2 and 3 results in N-terminal truncated (GLI1 $\Delta \mathrm{N})$ variant proteins (53). Interestingly, GLI $\Delta \mathrm{N}$ expression is downregulated in tumor tissue as compared to fulllength GLI1 (GLI1FL). GLI $\Delta$ N lacks the N-terminal SuFu binding motif, and is therefore resistant to the negative regulatory effects of Sufu, and also is resistant to the activity of dualspecificity tyrosine phosphorylation-regulated kinase 1 (Dyrk1). In mouse C3H10T1/2 cells, Dyrk1 acts to retain GLI1 in the nucleus, and may also enhance GLI1 transcriptional activity by other mechanisms (54).

An additional variant of GLI1, truncated GLI1 (tGLI1) lacks 123 base pairs corresponding to exon 3 and part of exon 4 (55). In contrast to the GLI1 $\Delta \mathrm{N}$ variants, tGLI1 was not detected in normal tissue, but highly upregulated in the tumor context of glioblastoma multiforme. Although tGLI1 nuclear localization and the transactivation of GLI binding sites was similar to GLI1, overexpression of tGLI1 led to distinguishing phenotypes. In comparison to GLI1FL, tGLI1 promoted increased migratory and invasive properties in vitro, and seemed to differentially promote CD24 expression in vitro and in vivo. tGLI1 was later detected in breast cancer cells, and found to promote additional factors such as vascular endothelial growth factor A (VEGF-A) (56). The differing expression and transcriptional regulation of target genes by tGLI1 may contribute to a number of cancer cell characteristics, accounting for some of the tumorigenic activity of Hh signaling (57). 


\section{GLI interactions and co-activators}

The C-terminal transactivation domain of GLI proteins was determined through deletion analysis, and characterized to interact with the human transcription factor IID (TFIID) TATA box-binding protein-associated factor (TAF9) (49). TAF9 is a subunit of the general transcription factor TFIID, which contributes to the preinitiation complex for RNA polymerase II (58). The interaction with TAF9 therefore provides a mechanistic basis for GLI-mediated transcriptional activation. This interaction may also be amenable to pharmacologic inhibition using the small molecule FN1-8 (59).

The functions of GLI proteins are also regulated by histone modifying and chromatin remodeling proteins. GLI1 acetylation at the K518 residue inhibits the GLI transactivation function (60). GLI2 is similarly acetylated. Deacetylation through enzymes such as histone deacetylase 1 (HDAC1) enhances GLI1 transcriptional activity. Degradation of HDAC1 via an E3 ligase complex containing Cullin3 and potassium channel tetramerization domain containing 11 (REN/KCTD11) could thereby promote GLI1 activity. Hh signaling suppressed this process, resulting in upregulation of HDAC1 levels and increasing the transactivation function of GLI1. Previously, loss of REN/KCTD11 activity had been implicated as a mechanism of Hh pathway activation in developing granule cell progenitors (61) and in medulloblastoma (62).

GLI3, but not GLI1, associates with the co-activator cAMP response element (CRE) binding protein (CBP). This interaction promotes the transcriptional activator function of GLI3 (39). This mechanism helps account for GLI3-mediated induction of GLII expression that is observed following stimulation with Hh ligands. In contrast, SKI protein acts with GLI3 as a co- 
repressor by recruiting HDAC enzymes. SKI cooperates with GLI3 to suppress the transcription of GLI1 (63). HDACs might act directly on GLI3, as seems to be the case with GLI1. Alternatively, the recruited HDACs could alter histone proteins and chromatin structure. An HDAC complex containing SAP18 and Sin3 can also negatively regulate GLI transcriptional activity, interestingly mediated by a nuclear function of Sufu (64).

GLI proteins are also implicated in chromatin remodeling through the ATP-dependent switch/ sucrose nonfermentable (SWI/SNF) complex. The interaction of GLI and the chromatin remodeling protein Snf5, an SWI/SNF complex member, along with other SWI/SNF proteins such as Smarcc2 and Smarce1 was detected through mass spectrometry (65). Snf5 did not interact with GLI2. Inactivation of SWI/SNF complex subunits Snf5 and Brgl resulted in enhanced GLI1 expression and activation of GLI target genes. The SWI/SNF complex in this way functions as a negative regulator of Hh signaling.

In addition to HDAC-related interactions, GLI proteins are also regulated by small ubiquitin-related modifier (SUMO) conjugation. GLI1 is likely subject to SUMOylation on the K180 and K815 residues, but GLI2 and GLI3 were also found to be SUMOylated (66). The SUMOylation of GLI2 and GLI3 were negatively regulated by protein kinase A (PKA) signaling, whereas GLI1 SUMOylation was less affected. GLI protein SUMOylation was mediated by the SUMO E3 ligase Pias1. Expression of wild-type Pias1, but not a Pias1 mutant lacking E3 ligase activity, enhanced GLI transcriptional activity. 


\section{Processing of Ci/GLI proteins}

In the absence of Hh ligand stimulation, Drosophila Ci protein is proteolytically cleaved, resulting in the $\mathrm{N}$-terminal repressor form $\mathrm{Ci}^{\mathrm{R}}$ (67). The cleaving of $\mathrm{Ci}$ is regulated by phosphorylation, involving kinases such as PKA, GSK3, and CK1. Ci then interacts with the E3 ubiquitin ligase $\mathrm{SCF}^{\mathrm{Slimb}}(30,68)$. Ci was later found to contain a processing determinant domain (PDD) that was critical for Ci to undergo processing instead of complete degradation in the proteasome. The transcription factor NF- $\mathrm{BB}$ is regulated similarly (69). Deletion of the PDD from $\mathrm{Ci}$ in Drosophila prevents the formation of $\mathrm{Ci}^{\mathrm{R}}(70)$, and $\mathrm{Ci}$ is instead completely degraded (71).

GLI3 in vertebrates seems to act similarly to Drosophila $\mathrm{Ci}$. GLI3 is subject to phosphorylation by PKA, GSK3, and CK1 $(72,73)$. GLI3 then interacts with $\mathrm{SCF}^{\beta-\operatorname{TrCP}}$, in which the F-box protein $\beta$-TrCP is homologous to Slimb in Drosophila(74). GLI2 is likely regulated in similar fashion by phosphorylation $(75,76)$. GLI2 is predominantly degraded by the proteasome, and not processed to truncated forms. This observation was explored by experiments with GLI2 and GLI3 chimeric proteins, which suggested that the GLI2 PDD was less efficient in preventing protein degradation (77).

Unlike GLI family members GLI2 and GLI3, and unlike the Drosophila Ci protein, GLI1 lacks a PDD as well as an N-terminal repressor domain. Therefore in the proteasome GLI1 does not undergo processing to create a cleaved repressor form. GLI1 exists as a potent transcriptional activator that is degraded by the proteasome. By linking ubiquitin to GLI1, $\beta$-TrCP directly destabilizes GLI1 by targeting GLI1 to the proteasome. The association of GLI1 and $\beta$-TrCP is 
therefore a determinant of GLI1 protein levels, and in this manner can regulate GLI1-dependent phenotypes including oncogenic effects. For this reason, the interaction between GLI1 and $\beta$ $\operatorname{TrCP}$ is a central focus of this dissertation.

$\mathrm{Ci} / \mathrm{GLI}$ proteins can also be substrates of $\mathrm{E} 3$ ubiquitin ligases other than $\mathrm{SCF}^{\mathrm{Slimb} / \beta-\mathrm{TrCP}}$. In Drosophila, Ci protein can interact with a Cul3-based E3 ligase complex through Roadkill (Rdx), also known as Hh-induced MATH and BTB domain-containing protein (Hib) $(78,79)$. In addition to UPS-mediated degradation, $\mathrm{Rdx}$ can also regulate nuclear entry of $\mathrm{Ci}(80)$. This dual function of $\mathrm{Rdx}$ is dependent upon $\mathrm{Su}(\mathrm{fu})$ levels (81). The vertebrate homologs of $\mathrm{Rdx}$ are Speckle-type POZ proteins (SPOPs), and the interaction of Ci/GLI with Rdx/SPOP is determined by conserved Ser/Thr (S/T)-rich motifs (82). Most cells in Drosophila lack primary cilia, and in vertebrates, Sufu-mediated GLI regulation through SPOP is cilium-independent (26). GLI1 protein, in comparison to GLI2 and GLI3, is a less efficient substrate for SPOP. Instead, GLI1 protein is regulated by the E3 ligase Itch, through interaction with Numb, in addition to the addition to the $\mathrm{SCF}^{\beta-\mathrm{TrCP}}$ interaction $(83,84)$.

\section{Genes regulated by GLI}

In addition to well known GLI targets such as GLII (39) and PTCHI (85), several studies have used chromatin immunoprecipitation (ChIP) and gene expression analyses to determine putative GLI transcriptional targets. In both granule neuron precursor cells and medulloblastoma, a malignancy characterized by oncogenic Hh pathway activation resulting from mutation of the PTCH1 tumor suppressor, hundreds of loci were bound by GLI1 (86). Similar methods and additional validation have revealed many GLI targets, including genes encoding the homeobox 
factors Nkx2.1 and Nkx2.2 (87). ChIP and transcriptional profiling of GLI3-bound sites in the limb buds of mouse embryos reveals suggested thousands of GLI-binding regions (GBRs) (88). The authors ultimately derived a core set of 205 Shh-responsive limb GLI target genes. Components of Wnt and TGF- $\beta$ signaling pathways were significantly enriched, in addition to Hh pathway components. Of these genes, 48 encoded known transcription factors. These targets included numerous homeobox factors of the HoxD family, and related cofactors such as Pbx, Meis1, and Meis2. Tbx transcription factors were also enriched.

Although the GLI-mediated regulation of some genes, such as Hh pathway components GLII and PTCH1, may be preserved in many cell types or "universal" (37), other GLI targets exhibit tissue specificity. These include transcription factor targets of Hh signaling. For example, GLI-mediated expression of Foxa2 is comparatively limited to processes of neural development (48). The prevalence of transcriptional regulators amongst the targets of GLI and Hh signaling reflect the crucial role of Hh signaling in the development of metazoans. As is described later, some Hh-responsive transcriptional targets of GLI are also involved in cancer, accounting for the oncogenic functions of GLI1 and GLI2.

\section{Hedgehog signal pathway in cancer}

The literature implicating Hh signaling in malignancy is extensive. Conceptually, oncogenic Hh signaling could occur through multiple mechanisms. There may be increased signaling through overproduction of Hh ligands, or mutation of pathway mediators such as PTCH, as is observed in basal cell carcinoma and medulloblastoma. Independently of Hh and Smo, other pathways such as RAS signaling can promote GLI protein activity. There are 
additional mechanisms of Hh pathway activation in some tumor types. For example, GLI1 is transcriptionally upregulated in Ewing sarcoma by a well-characterized fusion oncoprotein (8991). Like gliomas, some other forms of sarcoma can also exhibit gene amplification of $G L I$ $(43,92)$. This review will focus primarily on the role of the Hh pathway in pancreatic ductal adenocarcinoma (PDA). While Hh signaling seems to contribute to PDA, there is controversy as to whether the signaling is dependent upon hedgehog ligands. The role of Hh signaling in tumor cells versus stroma also remains unclear. As some of our data in the Results section concerns the cancer stem cell (CSC) population in PDA cells, the well-established role of Hh signaling in CSCs is also reviewed.

\section{Basal cell carcinoma and medulloblastoma: Archetypal Hh-driven malignancies}

Basal cell carcinoma is the most common type of cancer in humans, although advanced disease and metastasis are rare (93). The casual role of Hh signaling in this disease was identified through study of nevoid basal cell carcinoma syndrome (NBCCS), also known as basal cell nevus syndrome or Gorlin syndrome (94). NBCSS is an inherited condition affecting multiple organ systems, and patients are particularly prone to developing basal cell carcinomas (95). In comparison to sporadically occurring basal cell carcinoma, tumors associated with NBCCS may develop at a very early age, and in areas unrelated to sun exposure, such as the soles of the feet. Notably, patients are also predisposed to other tumors, including medulloblastoma. Identification of the tumor suppressor responsible for NBCCS led to the discovery of the human PTCH gene (96). Sporadic basal cell carcinoma is also highly associated with mutations that inactivate PTCH protein (97), and occasionally with activating mutations of Smo (98). Hh signaling is also frequently implicated in medulloblastoma through inactivation of the $\mathrm{PTCH}$, resulting in 
unrestricted Smo activation (99-103). The role of Smo-mediated Hh signaling in medulloblastoma and in basal cell carcinoma suggests the rational therapeutic use of Smo inhibitors, which is discussed separately.

\section{Hedgehog signaling in pancreatic ductal adenocarcinoma}

Reports from human specimens, as well as several model systems, including mouse models and cell lines, suggest Hh-GLI signaling has oncogenic functions in pancreatic ductal adenocarcinoma (PDA). The widespread Hh expression amongst pancreatic tumors and other gastrointestinal malignancies, and the lack of characteristic mutations of Ptch or Smo, suggested that Hh signaling in GI cancers may occur through the canonical Hh signaling pathway (104). In this respect, GI tumors are distinct from basal cell carcinoma and medulloblastoma, in which Hh signaling is constitutively activated through mutation of the Ptch or Smo receptors.

In pancreatic cancer, specific genetic lesions are highly prevalent, making PDA one of the best-defined cancer types (105). Activation of the small GTPase KRAS is highly prevalent, occurring in at least $95 \%$ of cases $(106,107)$. Additionally, mutations of the tumor suppressors p53 (108,109), INK4A (110), and SMAD4 (111) are common. Sequencing efforts have expanded the list of mutations associated with PDA (112). Still, the oncogenic activity of activated KRAS is central to the development of PDA, and as KRAS remains an elusive target for intervention, signaling pathways downstream of activated KRAS may offer better opportunities for pharmacologic inhibition (113). 
Many GEM models of pancreatic cancer are based on expression of activated KRAS. Mice with a Cre-inducible conditional allele $\left(L S L-\mathrm{KraS}^{G 12 D}\right)(114)$ at the endogenous Kras locus develop lesions resembling pancreatic intraepithelial neoplasia (PanIN) that progress to PDAlike tumors, albeit at low frequency (115). In these models, Cre recombinase is expressed under control of a pancreatic progenitor gene promoter, typically that of pancreatic and duodenal homeobox $1(P d x l)$ or pancreas transcription factor $1 \alpha(P t f l a)$, also known as $p 48$. This system results in expression of activated KRAS largely restricted to the pancreas. The combination of a loss-of-function allele, usually of either $\operatorname{Trp} 53$ or $\operatorname{Ink} 4 \mathrm{~A}$, with the $\mathrm{Kras}^{G 12 D}$ - based model greatly enhances PDA formation $(116,117)$. In addition to promoting PDA tumorigenesis, it has also been shown that expression of activated KRAS is necessary for tumor maintenance in this system (118).

Overexpression of Hh ligand is found in human PanIN and PDA (119), as well as in models based on the $\operatorname{Kras}^{G 12 D}$ allele (117). Additionally, a $P d x$-Shh mouse model results in proliferative lesions of the pancreatic ducts, and these structures were found to harbor Kras mutations and other PDA-relevant changes (119). Pancreatic epithelial expression of activated GLI2 (Pdx-CLEG2) likewise results in pancreatic tumors, although of undifferentiated histology (120). In support of the role of Hh signaling in PDA, therapeutic blockade of Hh signaling by cyclopamine or similar agents has efficacy in PDA cell lines and orthotopic PDA tumors $(121,122)$, and cyclopamine prolonged survival in a $\mathrm{Kras}^{G 12 D}$ - driven model (123). Hh signaling also cooperates with oncogenic Kras to promote PDA formation in mouse models $(120,124)$.

While these results implicated the overall Hh pathway in PDA, further investigation has suggested that upstream Hh pathway agents such as Smo inhibitors exert antitumor effects by 
acting on the tumor stroma. The responsiveness of cell lines representing PDA and other cancers to Smo inhibitors has been challenged (125), with evidence for a model of paracrine $\mathrm{Hh}$ signaling in cancer, in which tumor cells produce Hh ligands that act on stromal cells. In support of this hypothesis, genetic ablation of Smo in fibroblasts or treatment with Hh blocking antibody suppresses orthotopic tumor growth (125). Overexpression of Shh also enhances growth in vivo of pancreatic epithelial cells when co-injected with fibroblasts (126). There was evidence of $\mathrm{Hh}$ ligand-mediated signal transduction in the fibroblasts, as shown by accumulation of Smo in the primary cilia.

Similarly, treatment of $\mathrm{KraS}^{G / 2 D}$ - induced tumors with Hh inhibitor enhanced the response to the chemotherapeutic agent gemcitabine (127). In this case, the Hh inhibitor acted to reduce tumor stromal tissue, which had the effect of increasing the tumor vascular density and enhancing the concentration of gemcitabine within the tumor. In agreement with the paracrine model of Hh pathway activation in the stroma, expression of an activated Smo mutant in pancreatic epithelial cells did not seem to activate Hh-GLI signaling, and did not enhance $\operatorname{Kras}^{G 12 D}$-driven PDA formation (128). Interestingly, a recent report showed that small-molecule inhibition of stromal Hh signaling could actually accelerate PDA formation in mouse models, and suggested that the stromal tissue acted to restrain the progression of PDA (129). Collectively, these results suggest that targeting the classical Hh signal pathway in PDA mainly acts to restrict stromal cells, with consequences that may or may not be beneficial towards inhibiting tumor growth.

While it has been proposed that Hh signaling in PDA may be entirely limited to the tumor stromal compartment (128), more recent reports have argued for a direct role of Hh pathway 
components in PDA cells, albeit through Hh ligand-independent processes. As is discussed below, RAS and TGF $\beta$ signaling are suspected to promote GLI activity in PDA and possibly other tumors. Of these two mechanisms, the function of RAS signaling to activate GLI is best characterized. Conditional deletion of Smo does not impair Kras-driven PDA, and expression of Hh target genes is maintained in $\mathrm{Smo}^{-/-}, \mathrm{Kras}$-driven tumors (Fig. 4), suggesting a non-canonical means of GLI protein activation (34). One means of disrupting GLI signaling is through expression of a GLI3 mutant that exerts a dominant repressive function on GLI-mediated transcriptional activation (130). Expression of this dominant negative GLI3 protein does not impair normal development of the pancreas, suggesting that GLI transcriptional activation is dispensable in this context. In contrast, GLI antagonism by this method significantly delays pancreatic tumorigenesis in the $\operatorname{Kras}^{G 12 D}$ model. Additionally, ectopic expression of GLI1 in the pancreas accelerates formation of $\operatorname{Kras}^{G 12 D}$-driven tumors (Fig. 5). In mice homozygous for GLI1 loss-of-function alleles, tumor formation resultant from pancreatic expression of oncogenic Kras is significantly delayed (131).

In summary, current evidence suggests a multipart role for Hh signaling in PDA $(34,132)$. In this model, the GLI protein activity may contribute to PDA in both tumor and stromal cells, albeit through different mechanisms (Fig. 6). Ligand-dependent Hh signaling seems to occur in stromal cells, especially in fibroblasts. This mechanism helps reconcile evidence that PDA cells may overexpress Hh ligand to promote malignant progression, and yet be comparatively insensitive to ligand-dependent, Smo-mediated classical Hh signaling. In contrast, Hh ligandindependent activation of GLI proteins occurs in within PDA cells, and contributes towards KRAS-mediated tumorigenesis. As reviewed below, there are several reports that have shed light on RAS-GLI crosstalk mechanisms that may be active in PDA cells. These are relevant towards 
our Results (Chapter 2), which describe a mechanism of GLI1 stabilization involving the HMGbox factor SOX9.

\section{RAS and MAPK signaling: an alternate mechanism of GLI activation in cancer}

In some contexts, GLI protein activity can be activated through mechanisms independent from the classical Hh signaling pathway. In both PDA cells and in melanoma cells, GLI activity seems to be promoted by oncogenic RAS-MAPK signaling. These examples of Hh-RAS crosstalk may help explain the upregulation of GLI activity in cancers that lack upstream Hh mutations such as PTCH inactivation. Such interactions are intriguing from a therapeutic perspective, offering additional possibilities for targeting oncogenic GLI activity.

In PDA cells, GLI activity is partially dependent on activated KRAS and MAPK signaling (32). Transformation of nonmalignant human pancreatic ductal epithelial (HPDE) cells by KRAS ${ }^{\mathrm{G} 12 \mathrm{D}}$ results in upregulation of GLI transcriptional activity and GLII mRNA. In PDA cell lines, silencing of activated KRAS or treatment with MEK inhibitor decreased GLI transcriptional activity and GLII mRNA. In contrast, the phosphoinositide 3-kinase (PI3K) inhibitor wortmannin did not result in decreased GLI activity. In addition, expression of constitutively active BRAF, but not AKT, restored GLI signaling in PDA cells with knockdown of activated KRAS.

Hh ligand-independent GLI activity has also been demonstrated in the often-used $\operatorname{Kras}^{G 12 D}$ mouse model of pancreatic cancer (Fig. 4). In this result, the genetic ablation of Smo did not seem to affect normal development of pancreatic ductal or acinar structures, and did not 
affect development of PanIN-like lesions or PDA tumors (34). Intriguingly, expression of the Hh target genes Glil and Ptch was not decreased in the Smo-knockout tumors. In vitro, knockdown of Kras suppressed expression of Gli1 and Ptch mRNA in PDA cells.

Comparable results are found in melanoma cells. Melanoma is similar to PDA in that it is an aggressive malignancy frequently characterized by oncogenic activation of RAS-MAPK signaling (133). Hh pathway components are expressed in melanoma specimens and in tumors from an $N R A S^{Q 61 K} / \operatorname{Ink} 4 a^{-/-}$mouse melanoma model (33). While melanoma cells were sensitive to cyclopamine, this effect was synergistic with MEK inhibition. In melanoma cells, AKT signaling was also implicated in mediating RAS-GLI signaling, in contrast to reports using PDA cells (32). Notably, the effect of GLI1 transfection on GLI transcriptional reporter activity was highly synergistic with NRAS ${ }^{\mathrm{Q} 61 \mathrm{~K}}$ co-transfection in several cell lines.

\section{Hedgehog signaling and GLI proteins in cancer stem cells}

Cancer stem cells (CSCs), a subpopulation of tumor cells originally described in hematologic malignancies, are increasingly identified and characterized in solid tumors. Hh signaling is implicated in CSCs of many tumor types, including pancreatic adenocarcinoma. Unlike some other signaling mechanisms of CSCs, there are effective inhibitors of the Hh pathway, and some are approved for clinical use. Therapies that inhibit Hh signaling may therefore be a useful strategy to target CSCs in a variety of tumor types. This section reviews literature characterizing the role of Hh signaling in CSCs. Methods of targeting the Hh pathway are reviewed separately. 


\section{Cancer stem cells}

Cancer stem cells (CSC) are considered to represent a distinct population of cells within a tumor that have some characteristics of normal stem cells. The comparison of CSCs to normal stem cells includes the capacity of CSCs to undergo asymmetric division, facilitating self-renew and to differentiation into other types of tumor cell such as the transit-amplifying cells thought to make up most of a tumor population (134). Human CSCs can be functionally defined through xenograft assays, in which injected CSCs are able to recapitulate a tumor in an immunocompromised mouse model. Tumor cells can be serially transplanted, demonstrating self-renewal (135). Efforts to integrate the hierarchical model of CSCs in tumors with the clonal evolution model of tumor progression are ongoing, and it seems likely that both models will ultimately contribute to a common framework of cancer biology (136).

Cancer stem cells could possibly arise from the oncogenic transformation of normal stem cells, or from dedifferentiation of somatic cells (137). Evidence also exists for stochastic transitions within a cell population, in which cells may interconvert between CSC and non-CSC states (138). The biological significance of cells that function experimentally as CSCs is not without controversy $(139,140)$. Some skepticism arises from the considerable dependence of the CSC-like phenotype on the specific strain of host mouse, as has been shown for melanoma cells (141). Disagreement over what constitutes appropriate molecular markers of CSCs, poor understanding of the physiologic functions of these markers, inability to visualize CSCs in primary tumors, and the failure of many markers to generalize across multiple cell lines and tumors are all complicating factors in CSC research. 
Despite these caveats, further understanding of CSCs offers the potential to improve cancer outcomes. Many studies have shown CSCs to have increased resistance to therapeutic interventions as compared to control cells (142). For example, breast cancer CSCs may be resistant to chemotherapy (143), and glioma stem cells may exhibit radioresistance (144). This resistance of CSCs to therapy, along with the ability of CSCs to recapitulate a tumor, supports the hypothesis that CSCs may contribute to therapeutic failure and cancer relapse (145). Mechanisms currently understood to account for this resistance may involve pro- and antiapoptotic signaling pathways, DNA repair mechanisms, and expression of ATP-binding cassette (ABC) transporters (146). Other processes in CSCs such as aldehyde dehydrogenase (ALDH) expression, interaction with the microenvironment, and the epithelial-to-mesenchymal transition may also contribute to therapeutic resistance (147).

CSCs may also serve additional roles in malignancy. In particular, there is some evidence that CSCs may be implicated in dormancy (148), tumor cell dissemination (149), and overt metastasis (150). One intriguing possibility is that while CSCs may be resistant to conventional therapies, if specific CSC-related pathways can be determined, then some type of CSC targeted therapy might be devised (151-153). By targeting the cells that may contribute to therapeutic resistance and malignant progression, CSC-directed therapies offer the potential of increased survival.

\section{Role of Hedgehog pathway in cancer stem cells}

The Hh signaling pathway is highly implicated in CSCs, and consequently there is significant interest in Hh pathway inhibitors as a way of targeting CSCs $(154,155)$. The 
widespread involvement of Hh signaling in CSCs parallels the importance of the Hh pathway in normal stem cells (40). In model systems, there is evidence that inhibitors of Hh signaling disrupt activities associated with CSC function, including the serial passage of spheroids and grafted cells.

One context in which Hh signaling in CSCs is especially well studied is glial cell tumors, and glioma CSCs appear to have active Hh signaling. As shown by spheroid formation, an in vitro correlate of CSC activity, glioma CSCs are sensitive in a dose-response manner to the Smo inhibitor cyclopamine (156). Targeting of Smo through RNAi methods also decreased glioma CSC function in xenograft assays. Hh inhibition may exhibit synergistic effects with chemotherapy on glioma cell proliferation $(156,157)$. Other in vivo results using Hh-targeted therapy supports the contribution of Hh signaling to glioma tumor initiation $(158,159)$. Paracrine Shh signaling from endothelial cells may be responsible for Hh pathway activation in glioma CSCs (160).

In addition to glioma, Hh signaling has been studied in many additional tumor types. Reports indicate Hh-GLI activity contributes to CSC maintenance in cancers of the colon (161), stomach (162), and pancreas (121), as well as melanoma (163), chronic myeloid leukemia (CML) (164), and multiple myeloma (165), and also has tumorigenic effects suggestive of a CSC role in small cell lung cancer (166).

In breast cancer and lung squamous cell carcinoma, novel mechanisms have been identified in the function of Hh-GLI signaling in CSCs. Both GLI1 and GLI2 are upregulated in breast cancer cell spheroids, and Hh signaling maintains mammospheres in culture, as spheroids 
are increased by Hh pathway activation and decreased by cyclopamine treatment (167). In triple negative breast cancer (TNBC) cells, GLI1 promotes tumor initiation in a signaling pathway mediated by autocrine signaling through the VEGF receptor neuropilin 2 (168). This mechanism also involved RAS/MEK signaling, in agreement with the hypothesis that RAS may promote HhGLI activity. An autocrine signaling mechanism involving the Hh pathway also serves to maintain the CSC-like phenotype in lung squamous cell carcinoma (169). The transcription factor SOX2, when activated by PKCI, promotes transcription of hedgehog acetyl transferase (HHAT), an enzyme crucial to the production of Hh ligands. This mechanism enhances Hhdependent GLI activity in lung squamous cell carcinoma tumorspheres.

Consistent with the role of the Hh pathway in CSCs of diverse tumor types, the therapeutic inhibition of Hh signaling frequently has synergistic effects in combination with other chemotherapies (170-173). One mechanism by which Hh signaling may promote chemoresistance in tumor cells is through upregulation of $\mathrm{ABC}$ transporter proteins that mediate drug efflux (174).

\section{Therapeutic targeting of the Hedgehog pathway in cancer}

Hh pathway inhibitors show promise for treatment of human malignancy and are subject to ongoing research $(155,175-177)$. Small molecule inhibitors of Smo currently represent the most developed class of anti-Hh pathway agents (178), and have shown success in clinical trials. Still, only basal cell carcinoma and medulloblastoma have been shown to be responsive to Smo inhibitors in human trials, and even amongst these malignancies there is efficacy only in some 
patients. Drug toxicities and the development of resistance are also significant limitations of current anti-Hh therapies in clinical use.

\section{Methods of Hedgehog targeting}

The Hh pathway can be targeted at multiple levels. Most reported compounds that suppress Hh signaling either function as inhibitors of Smo, or are thought to act directly on GLI proteins. Strategies to disrupt Hh signaling at other steps of the pathway are also reported. The development of therapies that target Hh signaling involve testing compounds in cells to determine the effect on GLI transcriptional activity, followed by studies in animal models of Hhrelated malignancy, possibly leading to clinical trials. To date, clinical trials of therapies targeting the Hh pathway have focused on Smo inhibitors, and of these, only the agent vismodegib is currently approved by the FDA in an antineoplastic role.

\section{Smo inhibitors}

As a GPCR, Smo is a viable target for pharmacologic inhibition, such as by the naturally occurring compound cyclopamine (179). A number of Smo inhibitors have been developed with promising anticancer activity in preclinical studies. As of 2015, clinical trials are underway to determine the benefit of Smo inhibitors in a variety of cancers, including hematologic malignancies and solid tumors (155). Smo inhibitors have shown efficacy in treatment of basal cell carcinoma and of medulloblastoma. This review highlights some of the more promising results in the development of Smo inhibitors, especially with regards to the drug vismodegib. Additionally, there are other small molecule inhibitors of Smo that have been examined in 
clinical trials, or are currently under investigation $(154,155,178)$. It is therefore possible that additional Smo inhibitors will be added to the clinical repertoire of Hh-targeting agents in the near future.

\section{Smo inhibitors for treatment of basal cell carcinoma}

The Smo inhibitor vismodegib, or GDC-0449, has activity against basal cell carcinoma in both animal models and in clinical trials. Mice with the $P T C H^{+/-}$heterozygous genotype are prone to develop basal cell carcinoma-like cutaneous tumors, especially with exposure to UVB (180). Mice with overexpression of Shh (181), GLI1(182), or GLI2 (182) also develop similar tumors. Cyclopamine administration effectively prevents formation of basal cell carcinoma in the $\mathrm{PTCH}^{+/-}$UVB model (183).

Cyclopamine, because of unfavorable pharmacokinetics, toxicities, and other factors, is not a good candidate for systemic use in humans (184), although the development of cyclopamine analogs is a promising strategy (185). In contrast, topical formulations of Smo inhibitors have showed mixed success for basal cell carcinoma in clinical trials. Cyclopamine may be effective in this application (186). While the compound LDE225 was beneficial in some cases, CUR61414, a drug effective in a murine $P T C H^{+/-}$model, did not seem to affect human disease in a phase I trial (187).

Clinical trials have established the efficacy of oral vismodegib for basal cell carcinoma. In a phase I trial of vismodegib for locally advanced or metastatic basal cell carcinoma, a therapeutic response as determined by imaging or physical examination was established in 18 of 
33 patients (188). In another vismodegib phase I trial, a tumor response was observed in 20 of 33 patients with basal cell carcinoma (189). Aside from one unconfirmed response in a case of medulloblastoma, a tumor response was not seen in other solid tumors, including pancreatic cancer. A further phase I trial involving 33 patients with metastatic basal cell carcinoma established a response rate of $43 \%$ and median response duration of 7.6 months (190).

Given the apparent efficacy of vismodegib against sporadic basal cell carcinomas, a phase II trial sought to determine whether vismodegib would benefit patients with nevoid basal cell carcinoma syndrome (191). Under randomized, double-blind conditions, patients received vismodegib or placebo therapy. In this study, vismodegib was associated with a reduction in new basal cell carcinomas, as well as a decrease in size of existing lesions (Fig. 7). Additionally, the expression of Hh pathway target genes was suppressed by $90 \%$ in tumors. While vismodegib was effective in reducing basal cell carcinoma tumor burden and incidence in patients with basal cell nevus syndrome, the frequency of adverse events was also notable. Over half of patients discontinued therapy because of adverse events. Grade 1 and 2 adverse events were common and included muscle cramps, hair loss, weight loss, and loss of taste.

Vismodegib is approved by the FDA for use in recurrent, locally advanced, or metastatic basal cell carcinoma (192). Development of resistance to vismodegib therapy remains a significant problem (193). One mechanism of resistance is through mutation of Smo so as to prevent drug binding, as has been demonstrated in medulloblastoma (194). 


\section{Smo inhibitors for treatment of medulloblastoma}

Hh pathway inhibitors targeting Smo have shown potent antitumor activity in preclinical models of medulloblastoma. Mice homozygous for a conditional Ptch knockout, in which Ptch is deleted in cerebellar granular neuron precursor cells, develop medulloblastoma with $100 \%$ penetrance by at most 3 months of age (195). Hh pathway inhibitors can dramatically reduce disease progression in this model. In one study, all mice treated with the small molecule Smo inhibitor saridegib were alive at 80 weeks, whereas each mouse receiving vehicle control succumbed to progressive disease by 40 weeks (196). Notably, saridegib also showed activity against Smo protein that acquired resistance to vismodegib through a $\mathrm{D} 473 \mathrm{H}$ point mutation. Another small molecule Smo inhibitor, PF-5274857, similarly suppresses tumor progression in mice with primary medulloblastoma, and was shown to readily penetrate the blood brain barrier even in healthy animals (197). In comparison to other types of brain tumor, Hh pathway inhibition seems particularly effective against medulloblastoma cells, consistent with the mutational status of PTCH and the oncogenic role of Hh signaling in medulloblastoma (198).

Vismodegib has also shown efficacy in human patients with medulloblastoma. In one case, a 26 year old medulloblastoma patient with tumor-specific inactivating mutations of $P T C H$ showed dramatic, although transient, reduction in tumor burden as shown by radiography (199). The cause of the disease relapse was and traced to a new mutation of Smo, which abolished drug binding (194). NCT00939484, a phase II trial of vismodegib in the treatment of medulloblastoma, is ongoing with an estimated primary completion date of June 2015. 


\section{Inhibitors of GLI activity}

The compounds GANT61 and GANT58 were identified as small molecules capable of regulating a GLI transcriptional reporter (200). While belonging to distinct chemical classes, each compound exhibits dose-dependent interference with GLI1 and GLI2-mediated transcriptional activity. Similar screening methods using a library of naturally occurring compounds identified several inhibitors of GLI transcriptional activity, including physalin, an extract of the plant Physalis minima (201). Another small molecule inhibitor of GLI is FN1-8, which is thought to suppress GLI-mediated transcription by interfering with the interaction of GLI with the transcriptional co-activator TBP-associated factor 9 (TAF9) (59). Treatment with FN1-8 suppresses GLI transcriptional activity and also interferes with the colocalization of GLI and TAF9.

In contrast, the compound glabrescione B interferes with Hh signaling by binding to the zinc fingers of GLI1, thereby interfering with the interaction of GLI with DNA (202). As is generally true with Hh pathway inhibitors, glabrescione B has significant effects on CSC properties. Compound NMDA298-1, developed as a derivative of FN1-8, similarly inhibits GLImediated transcription, with an effect threefold greater for GLI1 as compared to GLI2 (203).

\section{Other Hedgehog pathway inhibitors}

In addition to inhibitors with Smo and agents that act on GLI proteins, there are a number of compounds that target Hh signaling in other ways. A monoclonal antibody against Shh may effectively suppress Hh signal transduction (204). The small molecule robotnikinin, in reference 
to the adversary of the fictional Shh namesake, may function in a similar mechanism (205).

Robotnikinin binds extracellular Shh with a $\mathrm{K}_{\mathrm{D}}$ of $3.1 \mu \mathrm{M}$ as determined by surface plasmon resonance (SPR). Itraconazole, an antifungal agent, prevents the ciliary accumulation of Smo that is part of normal Hh signal transduction (206). Itraconazole showed strong dose-dependent inhibition of GLI reporter activity and was effective in a mouse model of medulloblastoma. A phase II trial has also showed some activity of itraconazole against basal cell carcinoma (207). In addition, various other compounds such as arsenic trioxide (ATO) $(208,209)$ and vitamin D3 $(210,211)$ have been shown to inhibit Hh signaling through distinct mechanisms. ATO was effective in mouse tumors with drug-resistant Smo (208) a result in agreement with reported mechanisms involving ATO binding to GLI1 (209).

\section{Limitations of existing Hedgehog therapies}

The clinical utility of targeting Hh signaling is evidenced by the effectiveness of small molecule Smo inhibitors in basal cell carcinoma and medulloblastoma. Unfortunately, Smo inhibitors have not yet shown efficacy in other types of malignancy, including colorectal (212) and ovarian (213) carcinoma. A number of trials for other malignancies are ongoing (155). The failure of Smo antagonists in tumors thought to involve oncogenic Hh signaling may be due to the underlying mechanisms of Hh pathway activation. Mutations of the PTCH1 tumor suppressor are often characteristic of basal cell carcinoma $(96,97)$ and medulloblastoma (214). In contrast, GLI proteins may be activated by mechanisms independent of Hh and Smo in other malignancies, including pancreatic cancer, possibly explaining the failure of Smo inhibitors in this context. In this case, Hh pathway inhibitors that act downstream of Smo may prove useful, although there is as yet no clinical data. Increased understanding of the mechanisms governing 
GLI1 protein stability may help devise new strategies to target oncogenic Hh signaling. Destabilization of GLI1 protein could prove therapeutically useful in tumors resistant to Smo inhibitors, resultant either from intrinsic independence of Smo, or from acquired Smo mutations associated with resistance (194).

\section{The ubiquitin pathway and regulation of GLI1 protein stability}

The processing and degradation of GLI family proteins is regulated by the ubiquitinproteasome system. By interacting with the $\mathrm{SCF}^{\beta-\operatorname{TrCP}}$ complex, GLI proteins are ubiquitylated, targeting them to the $26 \mathrm{~S}$ proteasome. The association of GLI1 with the F-box protein $\beta$-TrCP is a critical subject of the Results section (Chapter 2), wherein we describe novel mechanisms governing GLI1 stability. The interaction of $\beta-\operatorname{TrCP}$ with the SCF component SKP1 also has an important role.

\section{The ubiquitin-proteasome system}

Protein degradation is highly selective and regulated, serving to control many cellular processes. The major pathway of protein degradation in eukaryotic cells is the ubiquitinproteasome system (UPS) (215). The discovery of the UPS is a significant achievement in biological chemistry, upending earlier paradigms of protein homeostasis (216). In this pathway, the small protein ubiquitin functions as a tag to achieve selectivity in the targeting of substrates to the proteasome. The UPS therefore involves two successive mechanisms (217). First, in a specialized type of posttranslational modification, proteins are marked with ubiquitin through a multistep mechanism. Secondly, ubiquitin-conjugated proteins are degraded by the $26 \mathrm{~S}$ 
proteasome, or in some cases processed without complete degradation. There are four ubiquitin genes in humans. In addition, several other small proteins are added as posttranslational modifications in a manner analogous to ubiquitin, although often with distinct functions.

A sequence of enzyme activities (Fig. 8) leads to the ubiquitylation of proteins (218). Ubiquitin is first activated by an E1 ubiquitin activating enzyme, leading to a thioester linkage connecting the C-terminal carboxyl group of ubiquitin to a cysteine residue of the E1 enzyme. The ubiquitin is then transferred to a cysteine residue of an E2 ubiquitin conjugating enzyme. Finally, an E3 ubiquitin ligase catalyzes the ubiquitylation of the target protein. Typically this ubiquitylation occurs via an isopeptide bond of ubiquitin to a lysine residue of the substrate.

A substrate that is monoubiquitylated can dissociate from the E3 ligase, in which case the monoubiquitylation likely serves to alter protein function rather than promoting degradation. Alternatively, the E3-bound substrate can receive additional ubiquitylation, as the ubiquitin peptide is also a suitable substrate. This mechanism leads to proteins modified by chains of ubiquitin. The function of polyubiquitin chains depends on the particular residue of ubiquitin involved in linkage. Some types of chains, such as ubiquitin K63 chains, may act to regulate protein function rather than targeting to the proteasome. In contrast, chains formed through residues such as K48 and K29 direct the polyubiquitylated substrate to the proteasome. The particular lysine residues involved determine the structure of the polyubiquitin chain, which affects the interaction with ubiquitin-binding domains present on other proteins $(219,220)$. For some substrates, ubiquitin receptor proteins are important in delivering the ubiquitylated substrate to the proteasome or otherwise determining the biological effects of ubiquitylation 
(221).The $19 \mathrm{~S}$ regulatory subunit of the mammalian $26 \mathrm{~S}$ proteasome recognizes ubiquitin tags and controls access to the core particle, which results in proteolysis (215).

The UPS regulates many pathways that are relevant towards human disease, and UPS dysregulation is implicated in pathologies such cancer, neurodegeneration, and other conditions $(217,222)$. Moreover, pharmacologic inhibition of the proteasome may be clinically useful in some malignancies $(223,224)$. In phase III trials, the proteasome inhibitor bortezomib was associated with increased response and survival in multiple myeloma (225) and mantle cell lymphoma (226), and bortezomib is approved by the FDA for treatment of these cancers. A second-generation proteasome inhibitor, carfilzomib, has also gained regulatory approval. Further pre-clinical and clinical studies for these and other proteasome inhibitors are in progress (227).

While current UPS therapies act to target the proteasome, abnormal functioning of the ubiquitin cascade is also highly linked to disease processes, including neoplasia. There is some evidence for the contribution of E2 ubiquitin-conjugating enzymes towards tumorigenesis (228), although most malignant alterations of the UPS in cancer seem to involve dysregulation of E3 ubiquitin ligases (229). Conceptually, an E3 ligase could promote tumor formation by ubiquitylating tumor suppressor proteins, or oppose tumorigenesis by targeting oncoproteins. In fact, many of the well-characterized E3 ligases target both oncoproteins and tumor suppressors, and therefore the overall role of the specific E3 ligase complex remains context-dependent, as is the case for the F-box protein and E3 ligase component $\beta$-TrCP $(229,230)$. 


\section{The E3 ubiquitin ligase}

In the ubiquitylation cascade, the E3 ligase functions as a substrate-specifying factor. This is reflected in the diversity of E3 ubiquitin ligases in comparison to E1 and E2 enzymes. Currently are at least 600 recognized E3 ubiquitin ligases, but only 30-some E2 enzymes and a smaller number of E1 enzymes (231-233). E3 enzymes can be broadly classified based on several structural motifs, which often relate to the mechanism of interaction with the E2 ubiquitin conjugating enzyme. The largest group of E3 ligases is of the RING-finger type, of which SCF complexes, including $\mathrm{SCF}^{\beta-\operatorname{TrCP}}$, belong (222). Other types of E3 ligase include the HECT, U-box, and PHD finger domains.

\section{SCF complexes}

The earliest described SCF complex was characterized in yeast, identifying the pathway responsible for degradation of the cyclin-dependent kinase (Cdk) inhibitor Sic1. In Saccharomyces cerevisiase, the degradation of Sic1 via the ubiquitin-conjugating (E2) enzyme Cdc34 allows the G1-S transition to occur (234). Extensive efforts have led to a model in which the Cdc34-mediated ubiquitylation of Sic1 occurs via an E3 ubiquitin ligase complex containing the proteins Skp1, Cdc53, Rbx1, and Cdc4 (235-242). This type of E3 ubiquitin ligase is known as an SCF complex, representing the proteins Skp1, Cdc53, and one of numerous F-box proteins.

The yeast protein $\mathrm{Cdc} 53$ is a cullin protein, and in metazoans the homologue of $\mathrm{Cdc} 53$ is CUL1. Therefore, in humans the SCF moniker denotes the three factors: SKIP1, CUL1, and an F-box protein. The SCF complex also includes the subunit RING box protein 1 (Rbx1). SCF 
complexes belong to a larger group of E3 ligases, known as the Cullin-RING family, which represent the largest family of E3 ubiquitin ligases (243). RING-based E3 ligase complexes are structurally and functionally distinct from the homologous to E6AP (HECT) domain-involving E3 ligases, in that RING-based E3 ligases such as SCF complexes do not form a ubiquitin thioester intermediate $(244,245)$. While SCF complexes are historically recognized for functions in the regulation of the cell cycle, the SCF family of E3 ubiquitin ligases also participates in other processes, including development, differentiation, apoptosis, an lipid metabolism (229).

A specific SCF complex is denoted by specifying the particular F-box protein. The SCF class of E3 ubiquitin ligases includes $\mathrm{SCF}^{\beta-\operatorname{TrCP}}$, which mediates the ubiquitylation of GLI proteins and other substrates. In brief, $\beta$-TrCP functions to recognize the SCF substrate, while SKP1 functions as an adaptor linking $\beta$-TrCP to CUL1, which in turn acts as a scaffold to link the $\beta$-TrCP/SKP1 complex with Rbx1. Through the RING domain, Rbx 1 mediates the interaction of an E2 ubiquitin-conjugating enzyme with the SCF complex. In this manner, the SCF promotes ubiquitylation of F-box substrates. As $\mathrm{SCF}^{\beta-\operatorname{TrCP}}$ is a key determinant of GLI protein stability, the structure and function of the SCF complex is reviewed in further detail.

\section{SKP1}

Human S-phase kinase-associated protein (SKP1) was first identified in a complex with cyclin A/Cdk2 and the F-box containing protein SKP2 (246). The homologue in yeast was then described (247). A small protein found in eukaryotes, SKP1 contains around 160 amino acid residues and functions as an adaptor protein to mediate the interaction of F-boxs protein with CUL1. SKP1 may also promote SCF complex assembly and function through other mechanisms. 
The expression of SKP1 serves to promote F-box protein expression by aiding in the correct folding and preventing formation of aggregates. Additionally, SKP1 expression promotes the substrate binding function of F-box proteins (248). The interaction of SKP1 and the F-box protein SKP2 has been determined by crystal structure (249). SKP1 and SKP2 directly associate through a complex interface that is highly hydrophobic, involving interactions of helical structures of both SKP1 and SKP2 in an interdigitated manner.

\section{CUL1}

Cullins are a family of proteins found in eukaryotes (250) that perform a scaffolding function in the structure of multiple E3 ubiquitin ligases (251). Cullin proteins are characterized by a C-terminal globular structure, known as the cullin homology domain, which mediates protein-protein interactions. Human CUL1 was identified as an interacting partner with the SKP1/SKP2 complex (252), and is now recognized as the cullin component of SCF complexes (243). Another example is the anaphase promoting complex (APC), a type of E3 ubiquitin ligase, which contains the subunit APC2 with cullin homology (253).

Cullin proteins are notable as substrates for neddylation, in which the ubiquitin-like protein NEDD8 (254) is covalently conjugated through mechanisms similar to the ubiquitin system (255). This posttranslational modification is important for the function of CUL1 in the SCF complex (256), and promotes the ubiquitination of the $\mathrm{SCF}^{\beta-\operatorname{TrCP}}$ substrate $\operatorname{I\kappa B} \alpha(257)$. Although only a small fraction of the cellular CUL1 is neddylated, the NEDD8-modified CUL1 may preferentially associate with $\beta$-TrCP (257). Unneddylated CUL1 is also bound by CAND1 
(258), which prevents CUL1-SKP1 association, thereby implicating CAND1 as a factor that regulates SCF complex assembly $(259,260)$.

The structure of CUL1 is critical towards the SCF complex function, and the crystal structure of $\mathrm{SCF}^{\mathrm{SKP} 2}$ in association with Rbx1 reveals the essential scaffolding role of CUL1 (261). In this structure, CUL1 interacts with each other element: SKP1, SKP2, and Rbx1. The SKP1/SKP2 site of interaction is located at the opposite end of CUL1 from the site of Rbx1 association. The N-terminal domain of CUL1 is a stalk-like structure composed of three repeats of a five-helix motif, of which the N-terminal repeat interacts with the SKP1/SKP2 complex. The C-terminal region of CUL1 is globular, and interacts with Rbx1. The rigidity of the CUL1 scaffold seems essential to the SCF complex function. Mutations that disrupted the rigidity of CUL1, in which the N-terminal and C-terminal CUL1 domains were connected by a flexible linker, disrupted the $\mathrm{E} 3$ ubiquitin ligase activity of $\mathrm{SCF}^{\mathrm{SKP} 2}$ (261).

\section{Rbx1}

Rbx1, also known as ROC1 or HRT1, contains a RING domain that is found in numerous human proteins. The RING domain was first identified in really interesting new gene 1 (RING1) and is a type of zinc finger structure $(262,263)$. While RING domain-containing proteins are widespread throughout eukaryotic organisms, performing a variety of roles, many RING proteins are crucial participants in the interplay of E2 ubiquitin-conjugating and E3 ubiquitin ligase complexes $(243,264,265)$. Indeed, almost all RING domain proteins are thought to have some function in the transfer of ubiquitin (244). In a yeast two-hybrid screen, the human ubiquitinconjugating enzyme E2 L3 (UBCE2L3) was found to associate with the RING protein human 
homolog of Ariadne (HHARI) (266). This structure was later determined by crystallography (267). In yeast, Rbx1 stimulates SCF ubiquitin ligase activity by promoting the association of Cdc53 with Cdc34 (242), which is homologous to the ubiquitin-conjugating enzyme E2R1 in humans(268).

A model of the interaction of the $\mathrm{SCF}^{\mathrm{SKP} 2}-\mathrm{Rbx} 1$ complex with UBCE2L3 has also been studied (261). UBCE2L3 interacts with the RING domain of Rbx1. With respect to CUL1, UBCE2L3 is situated in the same plane as the leucine-rich repeats of SKP2. Analogous in function to the WD-repeats of $\beta$-TrCP, the leucine-rich repeats of SKP2 determine substrate specificity. At a distance of about $50 \AA$, the active cysteine of the E2 enzyme is directly aligned with the tip of the leucine-rich region. The F-box protein, in this case SKP2, can interact with a number of different substrate proteins, each of which may contain multiple lysines or alternate residues to be conjugated with ubiquitin. From this standpoint, the structure of the SCF-Rbx 1 complex, and in particular the length of the CUL1 N-terminal region, serves to accommodate the diversity in F-box substrates (261).

\section{F-box proteins}

In 1995, the homology of protein Scon 2 was compared with the proteins $\beta$-TrCP, Cdc4, Met30, and FBXW2 (269). Each peptide sequence contained C-terminal WD repeats. Additionally, the analyzed sequences contained an N-terminal region of homology. This motif was later recognized to be a domain of interaction with Skp1 (235). 
The F-box motif, as the defining characteristic of F-box proteins, has around 50 residues. Only a few specific residues are conserved strongly (270). As SCF components, F-box proteins interact with SKP1 through the F-box motif, and F-box proteins found in SCF complexes also mediate SCF substrate recognition. This occurs through a region of the F-box protein that is distinct from the F-box domain. Typically, F-box proteins recognize substrates with site-specific phosphorylation of a small conserved sequence known as a destruction motif, or degron (264). The binding of F-box proteins to phosphodegrons of the substrate protein represents the canonical mechanism of substrate recruitment, although there alternative means of interaction (271). In addition to the role of F-box proteins in SCF complexes, some F-box proteins also participate in non-SCF protein interactions, in a variety of biochemical contexts (270).

In humans, there are several dozen F-box proteins $(272,273)$. F-box proteins often contain either a WD repeat region or a leucine rich repeat, but many F-box proteins contain neither motif. Moreover, the wide diversity of secondary structures found within various F-box proteins, which includes zinc fingers, leucine zippers, ring fingers, cyclin domains, proline-rich regions, and tetratricopeptide (TPR) repeats, is interpreted to suggest an evolutionary history in which the F-box motif has been transferred into various existing proteins (270). Bioinformatics analysis demonstrates that amongst human F-box proteins, examples containing either WDrepeat or leucine-rich repeats are most conserved in S. cerevisiae and C. elegans, whereas F-box proteins lacking either motif are frequently not conserved. Interestingly, C. elegans possesses around 10-fold more F-box proteins than do humans, many of which comprise F-box subclasses not found in either humans or the yeast $S$. cerevisiae (270). 
Formally, human F-box proteins may be named in reference to the substrate recognition domain: FBXL in the case of F-box proteins that contain leucine-rich repeats, FBXW for WD repeat-containing F-box proteins, and FBXO for F-box proteins not containing either leucinerich or WD-repeat motifs. Under this scheme, $\beta$-TrCP is represented as FBXW1. Most F-box proteins that participate in SCF complexes are either of the FBXW or the FBXL type, although some FBXO proteins might also associate as SCF complex members (272).

One way in which the ubiquitylation of SCF substrates is regulated is through assembly of the SCF complex. Intriguingly, some evidence suggests that substrate binding promotes SCF complex assembly (274). Conversely, SKP1 binding to the F-box domain of an F-box protein is thought to promote the interaction of the F-box protein with SCF substrates (248). These hypotheses are relevant to our studies of the interaction of $\mathrm{SCF}^{\beta-\mathrm{TrCP}}$ with the substrate GLI1. In the Results section (Chapter 2), we show that $\beta$-TrCP-SKP1 binding is altered in correlation with the interaction of $\beta-\operatorname{TrCP}$ with the substrate GLI1.

\section{$\beta-\operatorname{TrCP}$}

$\beta$-TrCP is the F-box protein component of the $\mathrm{E} 3$ ubiquitin ligase compex $\mathrm{SCF}^{\beta-\operatorname{TrCP}}$, and is homologous to the Drosophila protein supernumerary limbs (Slmb). In addition to the F-box motif, $\beta$-TrCP and Slmb contain a C-terminal region of WD40 repeats. The WD repeat is a sequence of approximately 40 residues, typically ending in tryptophan-aspartate (W-D). This motif was first identified in the peptide sequence of the $\beta$ subunit of retinal transducin, a heterotrimeric $\mathrm{G}$ protein. This sequence was immediately observed to have homology to yeast $\mathrm{CDC4}$, which is now recognized as a WD repeat-containing F-box protein (275). $\beta$ - $\mathrm{TrCP}$ is an 
abbreviation for $\beta$-transducin repeat containing protein, in recognition of the WD repeat homology. As the function of $\beta-\operatorname{TrCP}$ is that of an F-box protein, the systematic name FBXW1 may be preferable, in that it enumerates both F-box and WD structural features. In this dissertation, $\beta-\operatorname{TrCP}$ is used for consistency with existing literature.

Mammalian $\beta$-TrCP is subfamily of F-box proteins composed of $\beta$ - $\operatorname{TrCP} 1$ and $\beta$-TrCP2, although the existence of the latter protein seems frequently overlooked. In F-box nomenclature, $\beta-\operatorname{TrCP} 1$ is FBXW1 and $\beta$-TrCP2 is FBXW11. Differences in the $\mathrm{N}$-terminal sequences near the F-box motif are proposed to relate to the homo- and hetero-dimerization of $\beta-\operatorname{TrCP}$ proteins (276). Homodimerization of $\beta$-TrCP, mediated through the $\mathrm{N}$-terminal domains, has been associated with IкB $\alpha$ ubiquitylation (277). $\beta-\operatorname{TrCP} 1$ and $\beta$-TrCP2 appear to partially overlap in function. Some evidence suggests $\beta-\operatorname{TrCP} 1$ and $\beta-\operatorname{TrCP} 2$ exhibit functional redundancy. In a mouse model with $\beta$ - $\operatorname{Tr} C P 1$ loss-of-function, $\beta$ - $\operatorname{Tr} C P 1^{-/-}$male mice had defects in spermatogenesis associated with cell-cycle dysregulation, but there was no observed stabilization of the $\beta$-TrCP substrates I $\kappa \mathrm{B} \alpha$ or $\beta$-catenin (278). A contrasting report has demonstrated loss of I $\mathrm{B} \alpha$ degradation in $\beta$-TrCP1 knockout cells (279). Recently, proteomics methods using mass spectrometry were used to identify prospective $\beta-\operatorname{TrCP} 1$ substrates (280), as well as to compare $\beta$-TrCP1 and $\beta$-TrCP2 substrates (281). While most $\beta$-TrCP1 associated proteins, including those previously characterized as $\beta-\operatorname{TrCP} 1$ substrates, were also putative targets of $\beta$ - $\operatorname{TrCP} 2$, there were many proteins associated with $\beta$-TrCP2 that did not immunoprecipitate with $\beta$ - $\operatorname{TrCP} 1$. The functional consequences of these differences remain largely unknown.

As with other F-box proteins, substrates of $\beta$-TrCP are classically characterized by destruction motifs known as degrons. Degrons found in $\beta$-TrCP substrates are often of the form 
DSGXXS. Serine phosphorylation by specific kinases facilitates $\beta$-TrCP recognition, and ubiquitylation by $\mathrm{SCF}^{\beta-\operatorname{TrCP}}$ at lysines positioned 9-13 residues on the $\mathrm{N}$-terminal side of the degron $(230,276)$. Alternative degrons for $\beta$-TrCP have also been described (276). GLI1 does not possess classical degrons for $\beta$-TrCP, but does have DSGVEM sequences that appear to function similarly to the traditional DSGXXS degrons. This DSGVEM sequence is also found in GLI2 (35).

The structure of $\mathrm{SCF}^{\beta-\operatorname{TrCP}}$ interaction with the substrate $\beta$-catenin is partially solved. In this case, the crystal structure of $\beta$-TrCP-SKP1 bound to a 26 -residue $\beta$-catenin substrate peptide was determined (282). As with SKP2 (261), the F-box domain comprises multiple helices with linkers, forming a bipartite interface with SKP1. The propeller or torus-like structure composed of WD domains contains a central pore, partially entered by the critical degron residues of the $\beta$ catenin fragment. The Asp32 residue, of the $\beta$-catenin destruction motif DSGIHS, most extensively interacts with key residues of the $\beta$-TrCP WD structure (261). It is possible to recreate a full model of $\mathrm{SCF}^{\beta-\operatorname{TrCP}}$ bound to the $\beta$-catenin substrate peptide by combining the relevant structures of the $\beta$-TrCP-SKP1- $\beta$-catenin complex (282) with the previously reported $\mathrm{SCF}^{\mathrm{SKP} 2}$ structure (261). Notably, this structure places the substrate-binding WD propeller motif of $\beta$ - TrCP facing the E2 interacting site of Rbx1 (Fig. 9). In this manner, the WD domains of $\beta$ $\operatorname{TrCP}$, and the overall structure of $\mathrm{SCF}^{\beta-\mathrm{TrCP}}$, facilitate the transfer of ubiquitin from the $\mathrm{E} 2$ conjugating enzyme to the E3 ligase substrate. 


\section{Substrates of $\beta$-TrCP and function of $\beta$-TrCP as oncoprotein or tumor suppressor}

There are numerous reported substrates of $\beta$-TrCP, implicating $\mathrm{SCF}^{\beta-\operatorname{TrCP}}$ in the regulation of many biological processes. Cell cycle regulation and apoptosis are two functions incorporating multiple protein substrates of $\beta$-TrCP. Similarly, pathways such as NF- $\mathrm{kB}$ and growth hormone signaling involve multiple $\beta$-TrCP-targeted proteins (230). Krüppel-like factor 4(KLF4), the second topic of this dissertation, is also a reported substrate of $\beta-\operatorname{TrCP}(283)$. Compilations of verified $\beta$-TrCP substrates are available $(230,284)$, but new research quickly renders such listings outdated. In addition to true substrates, in which the $\beta$-TrCP-substrate interaction mediates substrate ubiquitylation by the $\mathrm{E} 3$ ligase $\mathrm{SCF}^{\beta-\operatorname{TrCP}}$, there is also at least one factor, heterogeneous nuclear ribonucleoprotein $\mathrm{U}(\mathrm{hnRNP}-\mathrm{U})$, that functions as a pseudosubstrate (285). The WD region of $\beta$-TrCP interacts readily with hnRNP-U, but this interaction does not affect hnRNP-U stability.

Rather than to list all reported $\beta$-TrCP substrates, the proposed functions of $\beta$ - $\operatorname{TrCP}$ in cancer are discussed, with several examples of $\beta$ - $\operatorname{TrCP}$ substrates that are implicated in malignancy. By targeting cancer-relevant proteins for degradation, or in some cases processing, $\beta-\operatorname{TrCP}$ can exert both pro- and anti- tumorigenic effects depending on context, although tumorpromoting roles seem to be emphasized in the literature $(229,230,276)$. The interaction of $\beta$ $\mathrm{TrCP}$ and GLI proteins is discussed separately, in greater detail. Proteomics and bioinformatics methods are increasingly utilized in the study of $\beta$ - $\operatorname{TrCP}$ substrates $(280,281)$, and are likely to expand knowledge of the function of $\mathrm{SCF}^{\beta-\operatorname{TrCP}}$ and other $\mathrm{E} 3$ ubiquitin ligases. 
The proteolysis of cell cycle regulators through the ubiquitin-proteasome system, involving multiple E3 ligases, is a central mechanism controlling the activity of cyclin-dependent kinases $(\mathrm{CDKs})$ and cell cycle progression. $\mathrm{SCF}^{\beta-\operatorname{TrCP}}$ can function to both positively and negatively regulate the cell cycle through multiple substrates (230). For example, $\beta$ - TrCP promotes degradation of CDC25A to inhibit CDK1 activity in S phase $(286,287)$, but can enhance CDK1 activity in G2-M phases by promoting degradation of WEE1 (288) and claspin (289,290). $\beta$-TrCP also promotes degradation of EMI (also known as FBX5/FBXO5) in mitosis (278). These substrates are also relevant to the function of $\beta-\mathrm{TrCP}$ in S-G2 cell cycle arrest in response to DNA damage (230). Increased degradation of WEE1 and claspin are proposed to explain the delay of the G2-M transition and mitosis in $\beta$-TrCP1 knockout MEFs (230).

A classic example of the role of $\beta$-TrCP in cancer-relevant signaling processes is through

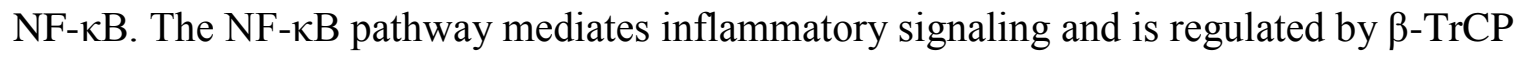
through I $\mathrm{K}$. $\beta$-TrCP recognizes phosphorylated $\mathrm{I} \kappa \mathrm{B} \alpha, \mathrm{I} \kappa \mathrm{B} \beta$, and $\mathrm{I} \kappa \mathrm{B} \varepsilon$ proteins, facilitating their ubiquitylation via $\mathrm{SCF}^{\beta-\operatorname{TrCP}}$ (291) (292-298). This ubiquitylation and subsequent degradation of

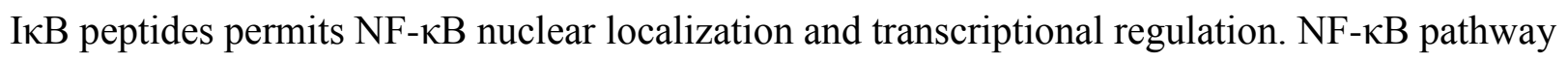
activation may contribute to malignancy (299), and overexpression of $\beta$ - TrCP is associated with

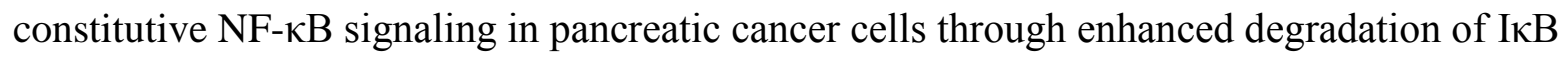
(300).

Other tumor types associated with upregulation of $\beta$-TrCP include breast cancer (301), colon cancer (302), melanoma (303,304), and hepatoblastoma (305). In mice overexpressing $\beta$ TrCP by use of the mouse mammary tumor virus (MMTV) promoter, there was abnormal development of the mammary gland related to increased epithelial proliferation, whereas the 
mammary glands of female $\beta-\operatorname{Tr} C P^{-/}$mice were hypoplastic (306). There was also increased nuclear and DNA-bound NF- $\kappa$ B in MMTV- $\beta-\operatorname{TrCP}$ mice, of which $38 \%$ developed tumors such as mammary, ovarian, and uterine carcinomas. In addition to $\mathrm{I} \kappa \mathrm{B}, \beta$-TrCP-mediated ubiquitylation exerts tumor-promoting effects by enhancing degradation of tumor suppressor substrates such as programmed cell death 4 (PDCD4) (307). By binding the eukaryotic translation initiation factor 4A1, PDCD4 suppresses translation, and has the phenotypic effects of suppressing proliferation and promoting apoptosis (308).

Although there is evidence that $\beta$-TrCP promotes tumorigenesis in some contexts, other well-known substrates of $\beta$ - TrCP are oncogenic in function, suggesting $\beta$-TrCP could have tumor-suppressive effects. One of the best characterized substrates of $\beta$ - $\operatorname{TrCP}$ is $\beta$-catenin. As a protein with dual functions in cell adhesion and transcriptional signaling, $\beta$-catenin has essential roles in development and other biological processes, and dysregulation of $\beta$-catenin signaling contributes to cancer development and progression (309,310). The Wnt signaling pathway regulates phosphorylation of $\beta$-catenin by kinases including $\operatorname{GSK} 3 \beta(311,312)$, which is "primed" through phosphorylation by CK1 (313-315). $\beta$-catenin contains a degron motif similar to that found in I $\mathrm{B}$ proteins, of the form DSGXXS, that is essential for recognition by $\beta$-TrCP (276). The $\beta$-catenin degron sequence is DSGIHS, of residues 32-37. Mutations of $\beta$-catenin that affect interaction with the degradation pathway components are found in some human tumors, although $\beta$-catenin stabilization through mutation of factors such as APC or overexpression of Wnt ligands is more common (316). Experimental suppression of $\beta$ - $\operatorname{TrCP}$ does result in $\beta$ catenin stabilization, but whether $\beta$-catenin stabilization in human tumors may occur through decreased $\beta$-TrCP activity is uncertain (276). 
Another example of a $\beta$-TrCP oncogenic substrate is the transcription factor SNAI1, often known as Snail, which is a potent suppressor of E-cadherin and inducer of EMT (317). In a pathway similar to that observed for $\beta$-catenin, phosphorylation by kinases including GSK3 $\beta$ promote association with $\beta$-TrCP, ubiquitylation, and degradation by the UPS $(318,319)$. The oncoprotein mouse double minute homolog $2(\mathrm{Mdm} 2)$, itself a type of E3 ubiquitin ligase, is also a $\beta$-TrCP substrate. Mdm2 functions as an E3 ligase for the tumor suppressor $\mathrm{p} 53$, promoting degradation of p53 (320). Increased $\beta$-TrCP-mediated turnover of Mdm2 in response to DNA damage leads to stabilization of p53, suggesting a tumor-suppressive function of $\beta$ - $\operatorname{TrCP}(321)$.

\section{Regulation of GLI proteins and $\beta$-TrCP interactions}

The GLI family of proteins, and the Drosophila homolog Ci are regulated by

phosphorylation, which governs interaction with $\mathrm{SCF}^{\beta-\operatorname{TrCP} / \mathrm{Slimb}} \cdot \mathrm{Ci}$ undergoes multisite phosphorylation by PKA (322), which seems to prime Ci for additional phosphorylation by GSK3 $\beta$ (323) and CK1 (324). In mammals, the related mechanisms are best understood for GLI2 and GLI3.

For GLI2 protein, a cluster of serine residues located within the carboxyl terminus is phosphorylated by PKA, leading to subsequent phosphorylation by GSK3 $\beta$ and CK1 (76). Phosphorylation of GLI2 enhances GLI2- $\mathrm{SCF}^{\beta-\operatorname{TrCP}}$ interaction, which inhibits the transcriptional activity of GLI2 (75). Notably, expression of GLI2 with phosphorylation site mutations can rescue some of the developmental consequences of Shh mutation (75). This result is consistent with the understanding of classical Hh signaling, in which Hh ligand binding regulates the 
stability and processing of the GLI/Ci effector proteins through site-specific phosphorylation and interaction with the $\mathrm{SCF}^{\beta-\mathrm{TrCP} / \mathrm{Slimb}}$ component of the UPS.

Highly similar processes also regulate GLI3 function, and the putative phosphorylation sites involved in GLI3 processing have been described using sequence analysis and mutagenesis (72). Serine phosphorylation in PKA consensus RRXS sites initiates the phosphorylation cascade. GSK3 $\beta$ targets serine residues for phosphorylation four residues N-terminal of a phosphorylated serine, and CK1 phosphorylates serines three residues on the C-terminal side of a phosphoserine. Thus, once initial priming phosphorylations by PKA have occurred, phosphorylation by GSK $3 \beta$ and CK1 can create additional GSK3 $\beta$ and CK1 consensus sequences, resulting in multisite phosphorylation.

The interaction of $\beta$-TrCP with the substrate GLI1 is less understood in comparison to GLI2 and GLI3. Although structural elements are thought to determine why proteasome does not process GLI1 into a truncated form, in contrast to GLI2/GLI3 and Ci (50), the regulation of GLI1- $\beta$-TrCP binding remains relatively enigmatic. Still, the association of $\beta-\operatorname{TrCP}$ and GLI1 is readily observed, and can be shown to destabilize GLI1 protein (35). A C-terminal DSGVEM motif seems to function as a degron in GLI1; this is notably distinct from the canonical DSGXXS degrons found in $\beta$-TrCP substrates such as I $\mathrm{B}$ proteins and $\beta$-catenin. Additionally, a conserved $\mathrm{N}$-terminal sequence acts to destabilize GLI1 through interaction with $\beta$-TrCP. Deletion of both motifs strongly stabilizes GLI1 protein, and mice expressing stabilized GLI1 in the skin develop lesions resembling basal cell carcinoma. 


\section{Part II: Krüppel-like factor 4}

\section{Part II overview: KLF4 and breast cancer metastasis}

Krüppel-like factor $4(\mathrm{KLF} 4)$ is a zinc finger transcription factor and regulator of proliferation, differentiation, and cell fate. KLF4 has important developmental roles, functions in the homeostasis of adult tissues, and is especially significant in pluripotency. The effects of KLF4 in cancer biology are varied, and while KLF4 may function as a tumor suppressor in some contexts, KLF4 expression promotes malignant properties in breast cancer cells. The role of KLF4 in the metastasis of breast cancer cells is poorly characterized. Although metastasis contributes heavily to morbidity and mortality in breast cancer, the biological mechanisms of metastasis are perhaps less understood in comparison to other hallmarks of malignancy. This introductory section reviews normal functions of KLF4, and describes the contrasting roles of KLF4 in breast cancer and other tumor types. Additionally, some of the translational aspects of metastasis research are discussed, and existing research into the role of KLF4 in metastasis is described in greater detail. In the Results section (Chapter 3), the function of KLF4 in the spontaneous metastasis of breast cancer cells is presented, in association with a signaling mechanism involving the collagen-binding discoidin domain receptor 1 (DDR1).

\section{Krüppel-like factor family}

Krüppel-like factors (KLFs) are transcription factors with diverse roles in biology, and are named for homology to the Krüppel gene found in Drosophila melanogaster (325). Common to Krüppel and KLFs are three conserved zinc fingers. There are 17 KLFs in the human genome, 
and KLFs are highly conserved in other mammalian species, including Mus musculus, Rattus norvegicus, Canis lupus, and Bos Taurus. Homologous KLF genes are described in the nonmammalian vertebrates such as Gallus gallus, Danio rerio, and Xenopus laevis, while the invertebrate Caenorhabditis elegans contains at least 3 KLFs (326). The three carboxy-terminal $\mathrm{C}_{2} \mathrm{H}_{2}$ zinc fingers found in Krüppel and in KLFs are also found in the transcription factor Sp1 (327). KLFs may then be classified as part of the Sp1/KLF family of zinc finger-containing transcription factors.

Krüppel was shown to regulate segmentation of the Drosophila embryo, and numerous functions are described for human KLFs. In addition to developmental roles, human KLFs are important regulators of cell proliferation, differentiation, and apoptosis (325). The KLFs are characterized by distinct expression and function in different cell types. KLFs 6, 10, and 11 are expressed widely. In contrast, KLF1 levels are high in erythrocytes, while KLF4 and KLF5 are abundantly expressed in gut tissue (328). Through the homologous carboxy-terminal zinc finger domains, KLFs interact with GC-rich elements of promoter and enhancer sequences to regulate gene expression. Amino-terminal sequences mediate interaction of KLFs with different protein binding partners, which helps determine whether the KLF serves to promote or to suppress expression of the target gene. For example, KLFs 3, 8, and 12 interact with the transcriptional corepressor CtBP (329-331).

\section{KLF4 discovery, expression, and structure}

The study of KLFs originates with the identification of loci that affect segmentation of the Drosophila melanogaster embryo (1). Mutations of these "gap" genes could result in loss of 
particular segments, and one such gap gene was named Krüppel, as loss could result in the "cripple" phenotype. All KLFs contain three $\mathrm{C}_{2} \mathrm{H}_{2}$ zinc fingers (Fig. 10). This motif is the most prevalent type of zinc finger, characterized by a $\beta \beta \alpha$ secondary structure containing two cysteine and two histidine residues that provide tetrahedral coordination of a zinc atom (332). In Krüppel and in KLFs, the spacing sequence between the zinc fingers is highly conserved, consisting of seven amino acids residues: TGEKP(Y/F)X, where $\mathrm{X}$ represents any residue. Aside from the three zinc fingers, and the intervening linker sequences, the homology between different KLFs is poor $(328,333)$. The KLF proteins recognize GC-rich DNA binding sites, and the sequence 5'CACCC-3' is a binding motif for KLF4 and other KLFs $(330,334,335)$.

The gene presently known as KLF4 was first reported in 1996, by two different groups. In one study, an NIH3T3 library-derived cDNA clone was found to interact in reducedstringency conditions with a probe composed of a zinc-finger DNA sequence from the transcription factor EGR-1 (336). The corresponding mRNA was most highly expressed in the colon, and so the gene was named gut-enriched Krüppel-like factor $(G K L F)$. The same gene was concurrently identified in primary mouse fibroblasts from E13.5 embryos, and named epithelial zinc finger $(E Z F)$ as high expression was observed in epithelial cells of the skin and other tissues (337). The homologous gene in humans was found in 1998, by screening a human umbilical vein endothelial cell (HUVEC) cDNA library with a probe derived from the zinc finger-coding region of $K L F 1$, previously known as erythroid $K L F(E K L F)$ (338). The tissue-specific naming of KLFs was eventually discarded in favor of sequential numbers, and GKLF/EZF became KLF4. 


\section{Expression of KLF4 in human tissue}

KLF4 is expressed to varying degrees in many tissues. In addition to the gut, skin, and vascular endothelial cells, KLF4 is expressed in the cornea (339), cardiac myocytes (340), lymphocytes (341), and thymus (342). Expression is also found in the lung and testis (336). In these reports, the comparatively high expression of KLF4 in postmitotic cells of the skin and gut suggested that KLF4 may serve to promote epithelial differentiation.

\section{Structure of KLF4}

In humans, the KLF4 gene is found on chromosome 9q31, spanning $6.3 \mathrm{~kb}$ and containing five exons (Fig. 11). The KLF4 transcript encodes a protein of 470 amino acid residues with a predicted $55 \mathrm{kDa}$ mass (338). Together, the three carboxy-terminal $\mathrm{C}_{2} \mathrm{H}_{2}$ zinc finger domains and spacers comprise 81 residues with strong homology to other KLFs and to the Drosophila protein Krüppel, and mediate DNA interaction. Adjacent to the zinc finger domains is a nuclear localization signal (NLS). Other functional domains of KLF4 protein are known. The $\mathrm{N}$-terminal region contains an acidic transactivation domain, while a repression domain is found in the middle region (338). Between the transcriptional activation and repression domains are two PEST sequences, which are rich in proline $(\mathrm{P})$, glutamate $(\mathrm{E})$, serine $(\mathrm{S})$, and threonine $(\mathrm{T})$. These elements are thought to promote degradation, possibly through the ubiquitin-proteasome pathway (343). 


\section{Transcriptional mechanisms of KLF4}

KLF4 protein contains functional domains that mediate transcriptional activation and repression. Similarly to other sequence-specific transcription factors, KLF4 may promote or suppresses transcription by recruiting other factors to the regulatory regions of target genes. These elements may include chromatin modifiers, which are enzymes regulating acetylation, phosphorylation, methylation, and ubiquitylation. KLF4 may also interact with chromatin remodeling complexes, which are ATP-dependent protein complexes that move or restructure nucleasomes.

\section{KLF4 as a transcriptional activator}

The transactivational role of the $\mathrm{N}$-terminal sequence was demonstrated through analysis of KLF4 mutant constructs. These experiments demonstrated that a fusion fragment containing 109 N-terminal residues with the GAL4 DNA-binding domain could activate a luciferase reporter, and compete with wild-type KLF4 (344). KLF4 was further shown through immunoprecipitation to interact with $\mathrm{CREB}$ binding protein $(\mathrm{CBP})$, a histone acetyl transferase (HAT). CBP, and the closely related protein p300, can act as transcriptional co-activators by catalyzing transfer of acetyl groups to positively charged residues of histone proteins. This is thought to promote transcription by loosening the chromatin structure and altering interaction with other regulatory proteins (345). Mutations designed to disrupt the KLF4-CBP interaction also suppressed KLF4 transactivation (344). KAT5, a HAT enzyme that also has histone deacetylase (HDAC) activity, may also interact with KLF4 (346). Other factors that function as co-activators with KLF4 include EGR2 and the p65/RelA subunit of NF- $\kappa B$ (347). 
Transactivation targets of KLF4 are diverse and include KLF4 (348), E-Cadherin (349), keratin 4 (350), keratin 19 (351), intestinal alkaline phosphatase (352,353), type 1 cGMP-dependent protein kinase (PKG I) (354), inducible nitric oxide synthase (347), Laminin- $\alpha$ 3A (355), Laminin- $\gamma 1$ (356), SPRR1A and SPRR2A (350), Involucrin (357), Lefty-1 (358), the stem cell factors Nanog, Oct4, Tbx3, and Sox2 (359), p2 $1^{\text {Waf1/Cip1 }}$ (360), p27 $7^{\text {Kip1 }}$ (361), p57 $7^{\text {Kip2 }}$ (362), Rb (361), miR-206 (363), platelet isoform of phosphofructokinase (PFKP) (364), and the urokinase receptor (UPAR) (365).

\section{KLF4 as a transcriptional suppressor}

KLF4 can repress transcription of target genes through competition with other factors. This mechanism of passive repression is illustrated through the competition between KLF4 and SP1, which share strong homology in the DNA binding domain. The binding of KLF4 to promoter regions inhibits SP1-mediated transactivation of genes such as SP1 (366), CYP1A1 (367), and HDC (346). KLF4 also possesses a central repression domain, which serves to negatively regulate transcription through recruitment of factors such as HDACs. The removal of acetyl groups from the histone proteins promotes closer interaction between positively charged histone residues and the negatively charged phosphates groups found in DNA, with the effect of suppressing transcription. For example, KLF4 mediates suppression of CD11d through recruitment of HDAC1 and HDAC2 (368), interacts with HDAC3 to suppress Cyclin B1 (369), and suppresses TP53 through HDAC1 and HDAC3 (370). In a complex with HDAC7 and KAT5, KLF4 binds the HDC gene promoter to suppress expression of HDC (346). KLF4 is also a negative transcriptional regulator of the Wnt signaling pathway. In this mechanism, KLF4 interacts with $\beta$-catenin/TCF-4 (371). Additional genes that are suppressed KLF4 at the 
transcriptional level include Bax (372), Cyclin D1 (373) and Cyclin D2 (374), Cyclin E (375), Fibroblast growth factor 5 (359), KLF2 (358,359), Lactate dehydrogenase (376), Laminin $\alpha 1$ (353), Nes (359), Ornithine decarboxylase (377), SM22 $\alpha$ (378), SM $\alpha$-actin (379), MAPK8/Jnk1 (380), N-Cadherin and Vimentin (380), and IFITM3 (381).

\section{Normal functions of KLF4}

KLF4 is implicated in several normal biological functions. These include cellular processes of proliferation, differentiation, and apoptosis. KLF4 can be induced by DNA damage and other types of stress. This is exemplified by KLF4 expression in the processes of growth arrest and differentiation as part of normal maintenance of the intestinal epithelium, or by the growth arrest and apoptotic resistance conferred by KLF4 upregulation in response to ionizing radiation. In concert with the role of KLF4 in maintaining normal epithelia, homozygous KLF4 knockout mice are born live but quickly perish from dehydration as the skin lacks an effective permeability barrier to moisture. Additionally, KLF4 has more recently been implicated in the biology of stem cells.

\section{Proliferation and differentiation}

KLF4 frequently has antiproliferative effects. This consideration is based on several lines of evidence, and mechanistically related to transcriptional effects of KLF4 on target genes that regulate cell cycle progression (325). Moreover, the antiproliferative role of KLF4 in epithelial cells is linked to cell differentiation. Observationally, KLF4 expression in epithelia is highest in differentiated, growth-arrested cells. Early reports of KLF4 expression in human tissue found 
that KLF4 mRNA expression in the colon was localized to cells undergoing growth arrest as part of differentiation into mature colonocytes (336). In contrast, proliferating cells at the base of the crypts had low expression of KLF4. These results demonstrate a gradient pattern of KLF4 expression in the colonic crypts, in which KLF4 upregulation is associated with decreased cell proliferation and increasing epithelial differentiation. In vitro, KLF4 expression corresponds to growth arrest resultant from serum starvation in NIH3T3 fibroblasts (336). In rat kidney epithelial cells (RK3E), KLF4 is upregulated in association with growth arrest when cultured cells become confluent (363). A similar effect is seen in the NIH3T3 line, where cells in complete media are subject to contact inhibition of growth in association with KLF4 upregulation if not passaged (336). In addition to the spatiotemporal association of KLF4 expression and growth-arrest, gain-of-function experiments implicate KLF4 as antiproliferative. Upon transfection of a KLF4 expression vector, cells expressing KLF4 failed to incorporate the nucleoside analog BrdU, whereas BrdU+ cells did not express KLF4 (336). As is documented in B lymphocytes, KLF4 is not invariably antiproliferative in function.

Multiple gene products mediate the antiproliferative activity of KLF4. The failure of DNA synthesis in KLF4-overexpressing cells (336) is further explained by analysis of cells containing an inducible KLF4 vector (382). In this system, ectopic expression of KLF4 was dependent upon a promoter responsive to the insect hormone ecdysone, and treatment with an ecdysone analogue blocked the G1/S phase transition. In subsequent work, expression profiling by microarray revealed a number of cell-cycle regulatory genes that were activated or suppressed by KLF4 (362). Upregulated products included the cyclin-dependent kinase inhibitors $\mathrm{p} 21^{\text {Waf1/Cip1 }}$ (383)and p57 $7^{\text {Kip2 }}$ (384), the insulin-like growth factor (IGF) signaling pathway antagonist IGFBP6 (385), and stratifin, a 14-3-3 protein that promotes G2 arrest (386). Increased 
expression of these antiproliferative genes in KLF4-overexpressing cells was verified by northern blot. In the same study, a number of proliferation-related genes were downregulated, including transcripts coding for Ki-67, CDK1, Cyclin D1, and Forkhead box protein M1 (FOXM1). In addition, KLF4 transcriptional profiling by microarray revealed a role for KLF4 as a negative regulator of certain macromolecule anabolic pathways, most notably protein biosynthesis (387). This function might conceivably also serve to inhibit proliferation. Collectively, these studies indicate that in many contexts KLF4 mediates an antiproliferative transcriptional program.

\section{Skin and gut development}

There are crucial functions of KLF4 in the differentiation of skin and gut tissues. The phenotypes of knockout mice are illustrative, as $\mathrm{Klf}_{4}{ }^{--}$mice exhibit deficiency of the skin moisture barrier that is fatal soon after birth (388). In normal development, the skin barrier forms at day 16 of gestation (389). The defect in barrier function is demonstrated by rapid passage of body fluids and permeability to external dyes (Fig. 12). The basal and spinous layers of the skin of newborn $\mathrm{Klf}^{-/}$mice appear normal, whereas electron microscopy of osmium tetroxide-fixed specimens shows abnormal distribution of lipids on the cornified envelope (CE) protein scaffold. KLF4 is also transcriptionally implicated in the protein kinase $\mathrm{C} \delta(\mathrm{PKC} \delta)$-related upregulation of involucrin in the differentiation of keratinocytes (357). In this mechanism, KLF4 expression is induced by $\mathrm{PKC} \delta(357,390)$.

Consistent with the role of KLF4 in the maintenance of the colonic epithelium, the number of goblet cells in the colon of Klf4 $4^{--}$mice is vastly decreased, (391). Remaining goblet 
cells appeared to have altered morphology (Fig. 13).The residual goblet cells were also abnormal by ultrastructural analysis and appeared to have defective secretory function with aberrant expression of $M u c 2$. Intestine-specific knockout of murine Klf4, from crossing Klf4-floxed mice with mice expressing Cre recombinase as controlled by the Villin promoter (392), revealed additional features. In addition to defects in goblet cell differentiation, abnormalities included increased proliferation and migration of intestinal cells, decreased brush-border expression of alkaline phosphatase, and decreased expression of carbonic anhydrase-1, a differentiation marker of colonic enterocytes (392). In the normal human colon, expression of KLF4 is lowest in proliferating cells at the base of the crypts (371). KLF4 protein becomes highly expressed at the apex, where the epithelial cells are differentiated and growth-arrested (Fig. 14).

KLF4 may also mediate some effects of the Notch signaling pathway on the differentiation of cells in the gastrointestinal tract. Treatment with a Notch pathway inhibitor increased the number of KLF4-positive cells in the crypts of the mouse small intestine and colon, and the amplifying crypt cells in control mice were replaced by differentiated goblet cells in the small intestine (393). Mechanistically, Notch signaling can suppress a response element in the human KLF4 promoter (393). The transcription factor Hairy and enhancer of split 1 (Hes1), a downstream target of Notch signaling, can also suppress KLF4 promoter activity (394), and Notch-mediated suppression of KLF4 may account for the proliferative and tumorigenic properties of Notch signaling in colon cancer cells (395).

Differentiation, metabolism, and apoptosis in colonic cells can be regulated by shortchain fatty acids such as butyrate, which may be obtained by bacterial fermentation of dietary fiber (396). These effects may be partially mediated through KLF4, as levels of KLF4 increased 
during butyrate-induced differentiation $(397,398)$, and butyrate can transactivate the KLF4 promoter (397). The actions of KLF4 to promote differentiation and suppress proliferation in cells of the intestine seem linked to the role of KLF4 as a tumor suppressor in gastrointestinal malignancy.

KLF4 also functions in other gastrointestinal tissues, including esophagus and stomach. In esophageal squamous epithelial cells, KLF4 is highly expressed and promotes a differentiated phenotype through transactivation of Keratin 4 (399) and Keratin 19 (351) promoters. Esophageal deletion of Klf4 in mice results in increased proliferation and defects in differentiation of esophageal cells (400). In the stomach, tissue-specific deletion of Klf4 results in mice with gastric epithelial hypertrophy, mucus cell hyperplasia, and partial loss of parietal cells (401). Although mice did not develop tumors up to one year of age, the altered epithelial differentiation, increased proliferation, and abnormal expression of acidic mucins were considered indicative of a premalignant state. It is possible that the changes in gastrointestinal epithelial tissue associated with Klf4 deletion may result from loss of the epithelial permeability barrier. In this respect, the tumor-suppressive effects of KLF4 in the mouse gut might not reflect the clonal origin of human tumors.

\section{Blood cells}

The role of KLF4 in regulating proliferation and differentiation is not limited to epithelial cells, and KLF4 expression seems to be important in various types of hematocytes. Although KLF1 is the KLF most associated with erythropoiesis (325), KLF4 is significantly upregulated in the HEL and K562 leukemic lines when these cells are induced towards erythroid differentiation 
(402). KLF4 also has significant functions in the development of B lymphocytes (325). KLF4 expression is upregulated in populations of pre-B cells (403). Mice with the tissue-specific knockout of Klf4, from the crossing of Klf4-loxP mice with CD19-Cre mice, have decreased numbers of pre-B cells and of mature B lymphocytes (374). In this study, the activation-induced proliferation of $\mathrm{Klf4}^{-/-}$B cells was impaired, with decreased proliferation into S phase. Klf4 was demonstrated to act directly on the cyclin D2 promoter. In contrast, downregulation of KLF4 in memory B cells is essential to the secondary antibody response, in which the memory B cells undergo rapid proliferation and differentiate into immunoglobulin-secreting plasma cells (404). KLF4 may additionally promote survival of natural killer (NK) cells (405).

KLF4 also regulates the development and function of monocytes. Although $\mathrm{Klf4}^{-/-}$ hematopoietic cells from fetal mouse livers could engraft equivalently to $\mathrm{Klf}^{+/+}$cells in lethally irradiated mice, there were interesting differences in the resulting monocytes populations in the chimeric system (406). While the resident $\mathrm{Klf4}^{-/-}$monocyte population was unchanged or modestly reduced in compartments such as bone marrow, spleen, and peripheral blood, the inflammatory monocytic population was almost completely depleted. The leukemia cell line HL60 is another notable model for the role of KLF4 in monocyte differentiation. Treatment with 12O-tetradecanoylphorbol-13-actetate (TPA) promotes macrophage-like differentiation in HL-60 cells, whereas all-trans-retinoic acid promotes differentiation to mature granulocytes. KLF4 overexpression in HL-60 cells induced expression of the monocytic markers CD11b and CD14 (407), and inducible expression of KLF4 potentiated differentiation into macrophages as induced by TPA (406). Suppression of KLF4 also enhanced HL-60 granulocyte differentiation (406). 


\section{Smooth muscle}

KLF4 suppresses transcription of the smooth muscle cell (SMC) markers, including smooth muscle 22-alpha (SM22 $\alpha$ ) and alpha smooth muscle actin ( $\alpha$-SMA), antagonizing the activity of transforming growth factor-beta (TGF $\beta$ ) signaling (378). This effect may be partly attributed to direct transcriptional suppression by KLF4. An additional mechanism, shown in fibroblasts, is based on interaction between KLF4 and Sma and MAD-related protein 3 (Smad3) (408). KLF4-Smad3 binding prevents Smad3, a key intracellular mediator of TGF $\beta$ signaling, from interacting with control elements of the SM22 $\alpha$ and $\alpha$-SMA genes. This interaction occurs through the Mad Homology $2(\mathrm{MH} 2)$ domain of Smad3. KLF4 therefore acts as a negative regulator of TGF $\beta$-dependent lung myofibroblast differentiation.

\section{Adipogenesis}

Experiments using the 3T3-L1 cell model of adipogenesis have implicated KLF4 in adipocyte differentiation (409). When stimulated with insulin, glucocorticoids, and the phosphodiesterases inhibitor 3-isobutyl-1-methylxanthine (IBMX), fibroblast-like 3T3-L1 preadipocytes adopt the morphology of mature adipocytes with accumulation of lipids (410). This involves early upregulation of the CCAAT-enhancer-binding proteins (C/EBP) including $\mathrm{C} / \mathrm{EBP} \beta$, a leucine zipper transcription factor that promotes peroxisome proliferator-activated receptor gamma (PPAR $\gamma$ ) expression (411). PPAR $\gamma$ transcriptionally regulates key metabolic pathways and is considered necessary and sufficient for adipocyte generation in fibroblasts (411). KLF4 expression is induced by exposure to the adipogenic cocktail, directly binds the $\mathrm{C} / \mathrm{EBP} \beta$ promoter, and can transactivate a $\mathrm{C} / \mathrm{EBP} \beta$ promoter in cooperation with early growth 
response protein 2 (EGR2) (409). These data characterize KLF4 as an early mediator of adipocyte differentiation.

\section{Neural development and regeneration}

Immature neurons in the central nervous system (CNS) are able to regenerate axons after injury, an ability that is lost in adult organisms. Of 111 candidate genes identified through expression profiling of developing retinal ganglion cells (RGCs), KLF4 transfection was most able to suppress axon growth (412). In mice with Klf4 knockout in RGC cells, there was increased axon growth and regeneration after experimental injury to the optic nerve. As with other processes, various additional KLFs provide complimentary or opposing functions to KLF4 in axon regeneration $(412,413)$. More recently, the negative effects of KLF4 on axon regeneration were linked to direct association of KLF4 with signal transducer and activator of transcription 3 (STAT3), suppressing STAT3 transcriptional activity (414). The implications of this STAT3 interaction towards KLF4 function in contexts such as tumor biology will be of interest. KLF4 also regulates the migration of neural progenitor cells in the developing cerebral cortex, and constitutive expression of KLF4 directed cells towards glial differentiation instead of developing into mature neurons (415).

\section{Stress response and inflammation}

KLF4 plays a critical role in the response of cells to various forms of stress. KLF4 expression is induced by treatment with methyl methanesulfonate (MMS) (360), an agent that causes DNA damage by interfering with the replication fork (416). Similarly, KLF4 is 
upregulated in response to DNA-damaging gamma radiation (417). KLF4 then directly bound the promoter of $\mathrm{p} 21^{\text {Wafl/Cip1 }}(360)$ in a $\mathrm{p} 53$-dependent manner, thus inhibiting cell-cycle progression. Therefore, the mechanisms by which KLF4 proliferation may apply not only to the context of normal epithelial differentiation, but also to the response of cells to DNA damage. The induction of KLF4 by genomic damage may also be modulated by the extent of damage, such that severe and irreversible damage results in KLF4 suppression and p53-mediated apoptosis (418). The roles of KLF4 in apoptosis are discussed later in the context of malignancy.

The function of KLF4 as a stress-response factor also extends to other forms of stress. KLF4 in vascular endothelial cells is induced by shear stress $(419,420)$. Furthermore, mice lacking endothelial expression of KLF4 are more sensitive to stress resultant from aortic banding (421). Interestingly, smooth muscle cells involved in human aortic aneurysms exhibit upregulation of KLF4, and experimentally induced aneurysms in perfused mouse aortas likewise showed significant elevation of KLF4 expression (422). By interfering with NF-kB functioning, KLF4 blocks TNF $\alpha$-mediated induction of vascular cell adhesion molecule 1 (Vcam1), suppressing the inflammatory response to vascular injury (423).

KLF4 serves to transcriptionally mediate the response of smooth muscle cells to injury or inflammation $(378,422,424,425)$. In normal blood vessels, KLF4 is minimally expressed, but is upregulated in the smooth muscle cells (SMC) of the tunica media after injury (425). The function of KLF4 in this context is to suppress the expression of genes that contribute towards SMC differentiation, and to promote expression of the anti-proliferative gene $\mathrm{p} 21^{\text {Wafl/Cip } 1}$. There is also evidence that oxidized phospholipids found in atherosclerotic lesions can induce KLF4 expression to suppress SMC markers, further implicating KLF4 in the pathogenesis of 
cardiovascular disease. In airway SMCs, KLF4 is upregulated by treatment with inflammatory cytokines including TNF $\alpha$, IFN $\gamma$, and interleukin 1-beta (IL-1 $\beta$ ) (426). This occurs through decreased expression of KLF4-targeting miR-25. KLF4 is also induced by inflammatory cytokines in HUVECs (420). KLF4 modulates these stimuli by differentially interacting with pro- and anti-inflammatory genes to exert anti-inflammatory and anticoagulation effects. KLF4 similarly suppresses VEGF transcriptional induction by the inflammatory transcription factor SAF-1 (427). In contrast, overexpression of KLF4 in the mouse esophageal epithelium promoted expression of several pro-inflammatory cytokines (428).

Macrophages can be activated by inflammatory factors including interferon-gamma (IFN $\gamma$ ), lipopolysaccharide (LPS), or tumor necrosis factor-alpha (TNF $\alpha$ ), and this activation is marked by induction of the enzyme inducible nitric oxide synthase (iNOS/NOS2) (429). KLF4 plays a critical role in this process (347). Treatment with IFN $\gamma$, LPS, or TNF $\alpha$ results in upregulation of KLF4, which cooperates with p65/RelA to directly promote expression of iNOS. In this context, KLF4 therefore acts in opposition to KLF2, which inhibits phagocytic activity and expression of inflammatory genes in monocytes and macrophages (430). Here, as in other contexts, different KLFs seem to exert opposing functions, and an imbalance between the opposing KLFs can be associated with disease. For example, monocyte expression of KLF2 was reduced in patients with atherosclerosis (430).

\section{Stem cells and pluripotency}

Pluripotent stem cells are able to differentiate into any of the three germ layers. Induced pluripotent stem (iPS) cells, in which adult cells are genetically reprogrammed to achieve 
pluripotency, are a major achievement in stem cell biology (431). A specific combination of factors including Oct3/4, c-Myc, Sox2, and KLF4 was able to create pluripotent cells from mouse embryonic or adult fibroblasts (432), and subsequently from human fibroblasts (433). The iPS cells have been shown to be indistinguishable in many ways from ES cells (434). Using a combination of small-molecule compounds, the minimum requirement can be reduced to only Oct4 and KLF4 (435). KLF4 may also be functionally replaced by the small molecule kinase inhibitor kenpaullone (436) in iPS cells (437).

Oct3/4, Sox2, and Nanog were previously considered core factors in pluripotency $(438,439)$. KLF4 could possibly act upstream to activate Nanog, as KLF4 may suppress p53 (440), which is a transcriptional suppressor of Nanog (441). KLF4 expression may also act as a counterbalance to c-Myc, in that KLF4 promotes $\mathrm{p} 21^{\text {Waf1/Cip1 }}$ to suppress proliferation (360), whereas c-Myc suppresses expression of p21 ${ }^{\text {Waf1/Cip1 }}$ (442). Additionally, suppression of p53 seems to be vital in rescuing cells from apoptosis induced by c-Myc overexpression (443), suggesting another possible role of KLF4. Later experiments provided evidence that KLF4 acts upstream of Oct4 and Sox2 in a transcriptional hierarchy (444).

KLF4 also regulates self-renewal and differentiation of embryonic stem (ES) cells. Prior to implantation, the blastocyst contains an inner cell mass (ICM) or embryoblast which later develops into the embryo. ES cells are harvested from the embryoblast, which involves destruction of the blastocyst. Leukemia inhibitory factor (LIF) is a cytokine expressed in embryos and used experimentally in stem cell research to inhibit differentiation. LIF treatment of murine ES cells induces expression of KLF4 and of suppressor of cytokine signaling-3 (SOCS-3) (445). While SOCS-3 seemed to negatively regulate LIF signaling, the role of KLF4 
upregulation was to block ES cell differentiation, and KLF4-overexpressing ES cells had greater capacity for self-renewal. Overexpression of KLF4 also reduces or eliminates LIF dependence (446,447). LIF induces KLF4 through STAT3 signaling (448). Ectopic expression of KLF4 can also revert cells derived from the mouse post-implantation epiblast (EpiSC) into ES-like cells (449). Mouse EpiSC cells differ in a number of ways from mouse ES cells, and may actually be more similar to human ES cells (450).

\section{KLF4 in the biology of cancer}

The role of KLF4 in cancer is complex and context-dependent. KLF4 acts as a tumor suppressor in some tissues. In particular, the functions of KLF4 to suppress proliferation and promote cellular differentiation help to oppose tumorigenesis in some gastrointestinal cancers. In contrast, the role of KLF4 in other cancers, including breast cancer, is less clear. Literature specific to the function of KLF4 in cancer metastasis is discussed separately in detail.

\section{Colorectal cancer and tumor suppressive functions of KLF4}

KLF4 was originally characterized as a gene associated with growth arrest in the colonic epithelium, and ectopic expression of KLF4 was seen to suppress synthesis of DNA (336). This

effect was further correlated in colon cancer cells with upregulation of $\mathrm{p} 21^{\text {Wafl/Cip1 }}$ and G1/S cell cycle arrest (382). The antiproliferative activity of KLF4 can be induced through DNA damage in colon cancer cells (417). KLF4 additionally suppresses proliferation in response to DNA damage by repressing cyclin B1 expression (451). The anticancer activity of investigatory compounds such as bis(3'-indolyl)methane (DIM) derivatives is also linked to KLF4-mediated 
growth arrest (452). In vitro, serum stimulation of HCT116 colon cancer cells promotes ubiquitin-mediated proteolytic degradation of KLF4 (453), providing another example of the common, albeit not universal, inverse relationship between KLF4 expression and cell proliferation. Introduction of KLF4 into the RKO colon cancer line, which is characterized by low levels of endogenous KLF4, results in growth arrest with an apoptosis-independent suppression of in vivo tumorigenicity (454). KLF4 also suppresses radiation-induced apoptosis in colon cancer cells (372), and in concert with this mechanism, has a radioprotective effect in the mouse colon (455).

\section{KLF4 expression in colon cancer}

Multiple independent studies have shown the expression of KLF4 to be decreased in colorectal carcinoma as compared with epithelial tissue of the normal colon $(373,456-459)$. In one study of 30 colon cancer specimens, KLF4 mRNA was downregulated nearly $50 \%$, with of loss of heterozygosity ( $\mathrm{LOH})$ in some samples (456). In a panel of six colon cancer cell lines, five exhibited LOH of the KLF4 gene, and expression of KLF4 protein and mRNA was decreased. Some of the cell lines also had KLF4 point mutations that suppressed the transactivation function of KLF4 on the $\mathrm{p} 21^{\text {Waf1/Cip1 }}$ promoter (456). These data suggest both the expression and activity of KLF4 are suppressed in colon cancer. A larger analysis of 125 specimens by RT-qPCR showed decreased expression of KLF4 mRNA in colon tumor samples, and also that KLF4 expression in normal tissues was correlated with patient survival (459). Expression of KLF4 in colon cancer tissue may also have prognostic value, as there is greater disease-free survival time in patients (Fig. 15) associated with tumor expression of KLF4 (458). KLF4 expression can also be related to the histologic grade of colon tumors. Whereas KLF4 
expression is low or undetectable in poorly-differentiated tumors, specimens of welldifferentiated tumors are more likely to demonstrate expression of KLF4 protein (460).

\section{KLF4- $\beta$-catenin pathway interaction}

In the human cancer syndrome familial adenomatous polyposis (FAP), and in multiple intestinal neoplasia (Min) mice, mutations of the adenomatous polyposis coli (APC) tumor suppressor lead to intestinal neoplasms. Compared to normal intestinal tissue, KLF4 expression is progressively downregulated in adenoma development in both FAP patients and Min mice (461). APC acts as a scaffold protein, associating with $\beta$-catenin and helping to promote $\beta$ catenin phosphorylation through enzymes such as glycogen synthase kinase 3 (GSK3). This phosphorylation marks $\beta$-catenin for degradation by the ubiquitin-proteasome system (462). The $\mathrm{Wnt} / \beta$-catenin pathway is central in the pathogenesis of colon cancer, in which mutations involving APC or other genes including $\beta$-catenin itself result in stabilization of $\beta$-catenin and oncogenic signaling by the Wnt/ $\beta$-catenin path (463).

Studies indicate that the tumor suppressive properties of KLF4 in colorectal cancer may occur in part through interaction and crosstalk with $\beta$-catenin signaling. Expression of wild-type APC results in upregulation of KLF4 in colon cancer cells, whereas expression of KLF4 blocks activity of a $\beta$-catenin-TCF reporter (464). One mechanism underlying the antagonistic effect of KLF4 on $\beta$-catenin signaling is the binding of KLF4 to the transactivation domain of $\beta$-catenin, which inhibits $\beta$-catenin-mediated transcription (371). Mice with intestinal-specific deletion of Klf4 have increased intestinal activation of Wnt target gene (392), consistent with a tumorsuppressive role of KLF4 in repressing $\beta$-catenin signaling. The inverse relationship of KLF4 
and $\beta$-catenin protein expression in gastric cancer suggest that KLF4- $\beta$-catenin crosstalk mechanisms may apply in this context as well (415).

\section{Other gastrointestinal malignancies}

The roles of KLF4 in other gastrointestinal carcinomas are often similar to those observed for colorectal cancer. Esophageal knockout of Klf4 in mice using the ED-L2 viral promoter led to increased basal cell proliferation, defective epithelial differentiation, and hypertrophy and dysplasia of the esophageal epithelium (400). Interestingly, esophageal overexpression of KLF4 led to increased inflammatory signaling, and suggests that KLF4 may exert disparate non-cell autonomous functions in tumorigenesis (428). As with colorectal cancer, KLF4 protein is decreased in human specimens of esophageal carcinoma, with a positive correlation of KLF4 expression and patient survival (465). Mice with gastric knockout of Klf4 have abnormal proliferation and differentiation of the gastric epithelium (Fig. 16), including premalignant changes that may lead to cancer (401), and as with colon cancer, human specimens of gastric adenocarcinoma lack expression of KLF4 $(401,466)$. These data suggest a tumor suppressive role for KLF4 in the context of gastric malignancy. Mechanisms of downregulation could include methylation and hemizygous deletion (466). Analysis of 49 human gastric carcinoma specimens by immunohistochemistry also showed decreased expression of KLF4 (467). KLF4 is similarly downregulated in hepatocellular carcinoma (468) and cytoplasmic KLF4 expression is associated with patient survival (469). Keratin promoter-linked deletion of Klf4 also supports a role of KLF4 in suppressing tumorigenesis of the oral epithelium (470). In

pancreatic cancer cells, KLF4 directly promotes $\mathrm{p} 27^{\mathrm{Kip} 1}$ expression, leading to suppression of 
tumor growth and metastasis (471). Pancreatic ductal carcinoma specimens frequently exhibit genomic loss of KLF4, with concomitant loss of KLF4 expression (472).

\section{Additional tumor-suppressive functions of KLF4}

In addition to effects KLF4 to suppress proliferation and promote differentiation, KLF4 may have other functions that inhibit tumorigenesis. Mouse embryonic fibroblasts (MEFs) with deletion of Klf4 exhibit genomic instability that is rescued by exogenous Klf4 expression (473). In contrast to wild-type MEFs, $K l f 4^{-/}$MEFs are capable of anchorage-independent growth (474). In murine hepatocellular carcinoma cells, KLF4 suppresses expression of Slug, leading to reversion of the epithelial-mesenchymal transition (EMT) (468); KLF4 may have a similar role in breast cancer (380) and ovarian cancer (475).

\section{Breast cancer}

While KLF4 seems to have tumor suppressive functions in colorectal cancer and several other types of gastrointestinal malignancies, the functions of KLF4 in the context of breast cancer is less clear. This uncertainty includes a lack of agreement on whether KLF4 mRNA expression is altered in breast cancer, and in which direction. Breast cancer is a diverse entity at the molecular level and, and KLF4 may have differing functions within the distinct subtypes of breast cancer, and also contribute variously towards the myriad of phenotypic alterations observed in mammary tumorigenesis. KLF4 seems to have antiproliferative activity in breast cancer cells, yet also helps to promote survival and resistance to anticancer agents. Additionally, KLF4 may help support the CSC population in breast cancer cells, and contributes towards 
tumor formation in some mouse models. As the central topic of this dissertation, literature on the function of KLF4 in the metastasis of breast cancer cells is reviewed separately in detail.

One hypothesis that may partially reconcile these differences is that $\mathrm{p} 21^{\mathrm{Waf} 1 / \mathrm{Cip} 1}$ mediates the balance of KLF4 function between tumor promoting and tumor suppressing effects (440). This work demonstrated that KLF4-mediated proliferation arrest in untransformed cells could be blocked by inactivation of cyclin D1 and p21. Additionally, p21 knockout in KLF4-expressing MEFs allowed cells to grow to a higher density, and form colonies lacking contact inhibition (Fig. 17). These data suggest that p21 may mediate tumor suppressive functions of KLF4, and in the absence of p21, KLF4 can exert tumor promoting effects.

\section{Expression of KLF4 in breast cancer}

Studies of KLF4 expression in human breast cancers are conflicting. Higher levels of KLF4 mRNA and protein are sometimes found in breast cancer in comparison to adjacent tissue (476). Amongst 146 human specimens, high nuclear expression of KLF4 by immunohistochemistry in breast cancer cells was associated with more aggressive clinical progression (477). In contrast, a large-scale RNA sequencing analysis of human breast tumors did not find increased KLF4 in comparison to normal tissue, and did not demonstrate prognostic significance of KLF4 $(478,479)$.

Expression of KLF4 in breast cancer model systems is also variable. Mammary tumor Klf4 expression in nine different genetically engineered mouse (GEM) models of breast cancer was decreased as compared to whole mouse mRNA $(479,480)$. Notably, GEM models of the 
luminal subtype of breast cancer exhibited the greatest decrease in Klf4 mRNA. In contrast, GEM models of breast cancer such as the C3(1)/TAg model had comparatively higher levels of Klf4. Analysis of KLF4 protein across multiple breast cancer cell lines also shows variable expression patterns $(479,481)$. In comparison to several breast cancer cell lines, KLF4 protein levels are higher in the nontransformed MCF-10A and MCF-12A cell lines (481).

\section{Functions of KLF4 in breast cancer cells}

Reports on the function of KLF4 in breast cancer cells have generally indicated that KLF4 inhibits proliferation and promotes survival in this context, although there is sometimes discordance between gain- and loss-of-function experiments. Evidence also indicates KLF4 can promote resistance of breast cancer cells to some targeted therapies. The anti-apoptotic effect of KLF4 also correlates with evidence that KLF4 expression promotes tumor formation of breast cancer cell lines in immunocompromised mouse models. KLF4 is also implicated in breast cancer stem cells.

\section{KLF4-dependent phenotypes in breast cancer cells}

Exogenous KLF4 may inhibit proliferation of 4T1 murine breast cancer cells, as shown by reduced BrdU incorporation (481). Similarly, KLF4 loss-of-function can enhance cell proliferation in MCF-7 cells (482). Mechanisms might include upregulation of antiproliferative genes, including $\mathrm{p} 21^{\mathrm{Waf} 1 / \mathrm{Cip} 1}$ as previously described (362). Additionally, KLF4 can also suppress estrogen dependent growth, by interacting with the estrogen receptor ER $\alpha$ and blocking induction of ER $\alpha$ target genes (482). In a variant of MDA-MB-231 cells selected for brain 
metastasis capability (231BrM), re-expression of KLF4 actually restored tumor growth from the inhibitory effects of miR-7 suppression (483). Overexpression of KLF4 alone did not affect tumor growth in this context.

\section{KLF4 promotes survival and drug resistance in breast cancer cells}

KLF4 has antiapoptotic functions in breast cancer cells. For example, suppression of endogenous KLF4 resulted in increased cell death in murine M6 cells, derived from the C3/TAg GEM model, and in nonmalignant human mammary epithelial (HMLE) cells (479). KLF4 overexpression by an adenoviral vector was shown in one study to increase apoptosis of 4T1 cells (481). In some cases, endogenous KLF4 helps breast cancer cells to form solid tumors in athymic mice, but does not substantially affect the growth of tumors that do form (484).

One process through which KLF4 may promote survival is through activation of mitogen-activated protein kinase (MAPK) signaling pathways. KLF4 promotes extracellular signal-regulated kinase (ERK) activity in breast cancer cell lines including MDA-MB-231 cells (485). The underlying mechanism was found to be that KLF4 promotes expression of miR-206 and miR-21, which enhance RAS-ERK signaling by targeting the RAS-ERK pathway suppressors SPRED1 and RASA1 (485).

Consistent with an apoptotic function of KLF4, protein and mRNA expression of the antiapoptotic factors BCL2, BCL-XL, and MCL1 were decreased following KLF4 knockdown (479). These effects appear related to a role of KLF4 in the resistance of breast cancer cells to therapies. Treatment of Her2-overexpressing BT474 with trastuzumab, a monoclonal antibody 
targeting Her2, or with lapatinib, a tyrosine kinase inhibitor affecting both Her2 and EGFR, resulted in KLF4 upregulation (479). Moreover, there was increased apoptosis in response to lapatinib when endogenous KLF4 was stably suppressed. This effect was additive with suppression of KLF5. The increased sensitivity of KLF4 knockdown cells to Her2-targeted therapy could also be rescued by re-expression of MCL1.

KLF4 may also play a role in the response of breast cancer cells to pharmacotherapies through the phenomenon of kinome reprogramming. In this process, cells are able to "escape" from the antiproliferative effects of MEK inhibitors through broad changes in kinase signaling, including upregulation of platelet-derived growth factor receptor $\beta$ (PDGFR $\beta$ ) (486). Subsequent research has revealed a function of KLF4 in kinome reprogramming and the response of breast cancer cells to MEK inhibition (in submission). KLF4 binds an element of the PDGFR $\beta$ promoter, and activates PDGFR $\beta$ transcription in the presence of MEK inhibitors. Furthermore, suppression of KLF4 prevents PDGFR $\beta$ upregulation and blocks the proliferative escape from MEK inhibition.

\section{KLF4 in breast cancer stem cells}

Several lines of evidence indicate a role of KLF4 in the breast cancer stem cell population. Mammospheres, which arise from mammary cells when grown in non-adherent conditions (487), have increased levels of KLF4 mRNA (488). Both Hoechst dye-excluding MCF-7 breast cancer cells and the population of CD44 $/ \mathrm{CD} 34^{-}$cells also express increased amounts of KLF4 (488). RNAi- mediated knockdown of KLF4 decreased the percentage of cells with CSC markers and also inhibited mammosphere formation (488). Additionally, KLF4 
mRNA expression is enhanced in ALDH-high cells from both breast cancer patient-derived xenografts (PDX) and from the C3/TAg model. KLF4 mRNA is similarly increased in C3/TAgderived cells grown as spheroids (484). Collectively, these data suggest a function of KLF4 in the breast cancer stem cell population. We have also observed increased KLF4 mRNA in the pancreatic cancer cell side population, or in pancreatic cancer cells grown as spheroids (Chapter 2).

The function of cancer stem cells is formally determined by the tumorigenic efficiency, in which decreasing numbers of cells are successively injected in immunocompromised mice to determine the minimum number of cells necessary for tumor formation $(135,489)$. It is not yet reported whether that KLF4 has a CSC function in breast cancer cells as defined by an in vivo limiting dilution tumor initiation assay. In contrast, miR-206 does appear to have in vivo CSC activity (484). As miR-206 expression in breast cancer cells is supported by endogenous KLF4 $(363,485)$, it seems possible that KLF4 could contribute to tumor initiation through miR-206.

One possible mechanism of KLF4 regulation in cancer stem cells is through Zeb1-miR200 signaling. An inhibitory, reciprocal feedback mechanism links Zeb1 and the miR-200 family $(490,491)$. Zeb1 and Zeb2 suppress transcription of E-cadherin, and several studies indicate that targeting of Zeb by miR-200 can result in upregulation of E-cadherin (492-496). miR-200c, miR203, and miR-183 inhibit expression of stem cell factors in cancer cells as well as mouse ES cells (497). The stem cell factors KLF4 and SOX2 are predicted targets of both miR-200c and miR203, and expression of either miRNA suppresses KLF4 levels. Downregulation of miR-200c, along with other miR-200 family transcripts, plays a critical role in the breast cancer stem cell 
phenotype(497), and could possibly account for the increased KLF4 that is observed in breast cancer stem cell populations.

\section{Metastasis}

In 2000, a seminal review (498) cited metastasis as "the last great frontier for exploratory cancer research.” The same authors, in a 2011 revision (499), commented with respect to metastasis research that "significant progress has been made." Despite some advances, many fundamental aspects of metastasis remain poorly understood, with increasing attention towards metastatic colonization as a particularly enigmatic process. Clinical metastases contribute greatly to the morbidity and mortality associated with cancer, ensuring the continuing importance of metastasis research.

\section{Metastatic breast cancer}

The incidence of breast cancer in the United States is estimated at 230,000 cases per year, with about 40,000 deaths (500). Breast cancer is the most common cause of cancer death for women in the United States between ages 20-59, and the second only to lung cancer in overall contribution to cancer mortality in women (500). Breast cancer prognosis is strongly related to staging (see below). In one analysis, five-year survival in stage IV disease, characterized by distant metastasis, was only $18 \%$ (501). In contrast, survival in breast cancer without distant metastasis was as high as $95 \%$ in the case of stage I disease. 
As is true for many other tumor types, breast cancer favors specific organs as sites of distant metastasis. Although breast cancer may potentially metastasize to nearly any location, common sites for breast cancer distant metastasis are bone, liver, and lung. Bone metastases are most common, and may be indolent and responsive to systemic therapies (502-504). Postmortem examination has revealed skeletal metastasis in 70-90\% of breast cancer patients (505). Bone metastases can also result in morbidity and mortality by causing pain and fractures. Some patients with isolated liver metastases may achieve long-term survival through metastasectomy, or surgical resection of metastasis (506). Similarly, resection of isolated pulmonary metastases may offer improved long-term survival (507-510). In this type of study, it is not always clear whether increased survival was a benefit of metastasectomy or from preoperative selection of patients (511).

Around $5 \%$ of patients develop brain metastasis $(512,513)$. The relatively low occurrence of brain metastasis overall reflects the tendency of breast cancer to be diagnosed at an early stage. In patients with metastatic breast cancer, $10-16 \%$ will develop brain metastasis $(512,514)$. Other studies have identified even higher rates of CNS involvement in patients with metastatic cancer (515). Also striking is the variation amongst subtypes of breast cancer, in which brain metastasis occurred in $0.7 \%$ of patients with luminal A tumors, $12 \%$ of patients with luminal B tumors, and $12 \%$ of patients with HER $2+$ breast cancer. In patients with triple-negative breast cancer, 7\% developed brain metastasis (516). 


\section{Survival in metastatic breast cancer}

In comparing patient cohorts across different time periods, survival in metastatic breast cancer is improving (517). Other cohort studies have associated survival improvements in metastatic disease with the introduction of taxane therapy (518). Hormonal therapies and trastuzumab may also have contributed to longer survival in metastatic breast cancer (519). Despite these advances, long-term remission is only achieved by a small percentage of patients with metastatic disease (520). In a review of 36 randomized trials with 13,083 total patients between years 1999-2009, overall survival for women starting chemotherapy for metastatic breast cancer was less than two years (521). Most patients who develop breast cancer metastases were originally diagnosed with early-stage disease. In one study, only $23 \%$ of patients with metastatic breast cancer had initially presented with metastatic disease. Overall survival, measured from the start of first-line chemotherapy for metastatic breast cancer (Fig. 18), closely adhered to an exponential curve with median survival of 20.4 months (522).

\section{Model systems for triple-negative and claudin-low breast cancer}

Overall, about 13\% of breast tumors are triple-negative, lacking expression of estrogen receptor (ER) and progesterone receptor (PR), and without overexpression of HER2 (523). Triple-negative disease, which accounts for much of the basal-like intrinsic subtype of breast cancer (524), is overrepresented among African-Americans (525). The basal-like intrinsic subtype of breast cancer, which exhibit an expression profile similar to that of basal breast epithelial cells(526), is associated with more aggressive disease and a poorer prognosis (527529). While basal-like breast cancer does not clinically appear to have an inherent resistance to 
chemotherapy as compared to other subtypes, the decreased survival associated basal-like disease may be related to relapse among patients without complete response to therapy (530).

In addition to luminal A, luminal B, HER2-enriched, and basal-like intrinsic subtypes (526), a "claudin-low" subtype was later identified (480). Claudin-low tumors are named owing to low expression of claudins 3, 4, and 7. Claudins are small transmembrane proteins of 20-27 $\mathrm{kDa}$ that are critical components of tight junctions, thereby controlling paracellular transport (531). The claudin-low subtype is also characterized by low expression of occludin, another component of tight junctions, as well as E-cadherin. Expression of luminal genes is low, and while claudin-low tumors may represent a subset of basal-like disease, expression of the basal cluster is irregular (480). In contrast, claudin-low breast cancer cells seem to be enriched for CSC and EMT characteristics (532-535). Gene expression in claudin-low cell lines also clusters with human mesenchymal stem cells (hMSCs) (536).

While claudin-low tumors represent a small subset of human breast cancer, comprising $12 \%$ of tumors in one data set (534), cell lines that cluster with the claudin-low subtype seem to be disproportionately represented in breast cancer research (536). In contrast, the luminal A subtype, mostly comprising low grade ER+ tumors, accounts for around 70\% of human breast cancer and is yet apparently unrepresented by any cell line (536) or transgenic mouse model (480). One hypothesis to explain the discrepancy between the epidemiology of human breast cancer subtypes and the gene expression patterns of established cell lines is that of a selection bias conferred by the conditions of cell culture. For example, the passage of cells in culture would disfavor outgrowth of luminal A-type cells with low proliferation and stronger attachment (536). 
The breast cancer cell lines MDA-MB-231 and MDA-MB-435, which are notable for the ability to form spontaneous metastases in immunocompromised mice $(537,538)$, cluster with human claudin-low tumors (534). The C3/TAg GEM model of breast cancer was developed using a transgene containing the early region of simian virus 40 large tumor antigen (TAg) and a regulatory region of the rat prostatic steroid binding protein $[C 3(1)]$ gene $(539,540)$. Female C3/TAg mice develop mammary carcinoma with $100 \%$ penetrance by 6 months of age, whereas males develop carcinoma of the prostate. Pulmonary metastases reportedly occur in $15 \%$ of C3/TAg female mice with mammary tumors (540). In unsupervised hierarchical clustering analysis, C3/TAg mammary tumors exhibit significant overlap with basal-like breast cancer in humans (480). The M6 cell line was derived from C3/TAg mouse mammary tumors (541).

\section{The metastatic cascade}

The development of metastasis may be described sequentially, consisting of discrete stages of progression (542-545). At each stage, specific functions help cells overcome the numerous obstacles to metastasis (Fig. 19). Once a tumor is established, cellular proliferation must continue, with sufficient angiogenesis to support ongoing tumor growth. Using invasive processes or other mechanisms, cancer cells must gain access to the vasculature, possibly through the lymphatic system or by direct access to blood vessels. Malignant cells may then enter into circulation. Cells that survive transit through the vasculature may lodge in capillaries of distant organs, perhaps adhering to endothelial c ells or subendothelial structures. Cancer cells may exit the vasculature through extravasation or other alternative mechanisms, or possibly rupture the capillary vessel in which the cancer cells are arrested through continued proliferation. In principle, metastasis can be inhibited by targeting any step of the process $(542,546)$. 
From the sequence of steps necessary for successful metastasis, it could be questioned whether some steps have rate-limiting functions. Increasingly, the ability of tumor cells to survive and proliferate are viewed as bottlenecks in the metastatic cascade, explaining why large numbers of circulating tumor cells may only result in a small number of macrometastases $(542,545,547,548)$. Current cancer staging standards define macrometastases as greater than $2.0 \mathrm{~mm}$ in diameter (549). Micrometastases have a diameter greater than $0.2 \mathrm{~mm}$ and/or more than 200 cells in cross section, but are less than $2.0 \mathrm{~mm}$. One estimation is that less than $1 \%$ of micrometastases will further develop into macrometastases (550). Single cells or cell clusters less than $0.2 \mathrm{~mm}$, or a cluster of fewer than 200 cells in cross section, are defined as isolated tumor cells (ITCs). Lymph nodes containing only ITCs are not considered positive lymph nodes (549).

\section{Circulating tumor cells}

Circulating tumor cells (CTCs) represent an intermediate stage of metastasis, dividing between early events in the metastatic cascade found at the site of the primary tumor, and later events at the site of distant organs (Fig. 19 and 20). Although CTCs are often difficult to isolate or quantify, as they are rare in comparison to normal blood cells, these cells are of particular interest for several reasons. CTCs may have clinical prognostic and predictive significance, and are increasingly used to aid investigation into the underlying mechanisms of cancer metastasis. CTCs could possibly also arise as cells shed from metastases, although the literature addressing this hypothesis is sparse (551). In contrast, the re-seeding of primary tumors in animal models is documented $(552,553)$. 
Importantly, the identification and quantification of CTCs has been shown to have prognostic significance in clinical studies. CTC presence is associated with poorer prognosis in both early, non-metastatic breast cancer (554-556), and in the context of metastatic breast cancer (557-561). This result was also reproducible in a study specific to triple-negative breast cancer, as CTCs as determined by two different methods were correlated with reduced time to progression (TTP) and overall survival (OS) (562). The prognostic value of CTCs in breast cancer is also supported by a meta-analysis of 49 studies with over 6,000 total patients (563). Some data suggest CTC measurement may actually be a superior to radiographic methods in assessing disease progression (564). CTCs may have prognostic significance in other malignancies, including prostate cancer (565), melanoma (566), and colorectal carcinoma (567).

CTCs can potentially provide more information than simple quantification. CTCs can also be isolated for analysis, and single-cell analysis of CTCs is an increasingly viable approach (568) that may provide useful insight into metastatic phenomena, and may possibly lead to improved guidance of therapy (569). CTC characterization is proposed to serve as a "liquid biopsy" to diagnose malignancy and monitor disease course (570). Single-cell analysis has shown that the CTCs in human breast cancer are heterogeneous, and sometimes fail to cluster based on case ID (571).

\section{Metastasis suppressors}

In 1988, a novel gene was identified based on differential mRNA expression amongst cell lines corresponding to metastatic potential (572). This gene, originally named NM23 and now known as NME1, codes for the enzyme nucleoside diphosphate kinase A and was the first 
identified of the class of genes known as metastasis suppressors. Expression of NME1 in cancer cells does not impair tumorigenesis in immunocompromised mice but suppresses formation of metastasis (573). These observations have led to the concept of the metastasis suppressor: "Metastasis-suppressor genes suppress the formation of spontaneous, macroscopic metastasis without affecting the growth rate of the primary tumor" (574). A growing list of metastasis suppressors are now recognized, including many in breast cancer (575). Metastasis suppressors are functionally defined based on animal models, and so whether a protein functions as a metastasis suppressor may depend on the chosen model system.

\section{Animal models of metastasis}

Models of tumor metastasis can take several forms $(544,576)$. Spontaneous metastases occur secondarily to primary malignancy. This primary tumor could be arise from orthotopically injected cancer cells, or develop from normal tissue as autochthonous disease. In the latter case, tumor development could be prompted by means such as genetic engineering or chemical carcinogens. Another type of assay, experimental metastasis, involves direct inoculation of cancer cells into circulation. In reference to the metastatic cascade, the spontaneous metastasis assay replicates more steps of human cancer progression than does an experimental metastasis assay (576).

In the Results section (Chapter 3), we describe the function of KLF4 in a spontaneous model of breast cancer metastasis using NOD/SCID/ $\gamma$ (NSG) mice. Mutation of the DNAdependent protein kinase catalytic subunit results in severe combined immunodeficiency (SCID), with defects in both adaptive and innate immunity including lack of mature B and T lymphocytes 
(577). The NOD.CB17-Prkdc ${ }^{\text {scid }}$ (NOD/SCID) model was created through backcrossing CB17$\operatorname{Prkdc}^{\text {scid }}$ (SCID) mice into the NOD/Lt strain (578). While SCID mice retain natural killer (NK) cell activity and complement, NOD/Lt mice are have functional deficits in NK cells and antigenpresenting cells (APC), and lack circulating complement $(579,580)$. NOD/SCID mice exhibit immune defects of both models, and are characterized by absence of mature B and $\mathrm{T}$ lymphocytes, lack of complement, and functional defects in NK cells and in macrophage populations (578). In addition, NOD/SCID mice do not develop immune mediated, insulindependent diabetes mellitus, which is a characteristic of non-obese diabetic (NOD) mice.

The immune system of the NOD/SCID/ $\gamma$ (NSG) mouse is further altered through loss of the interleukin-2 receptor gamma chain (IL-2R $\gamma$ ) (581). This peptide, also called the common gamma chain $\left(\gamma_{c}\right)$ or CD132, is additionally a component of receptors for other interleukins (582), including IL-4 (583), IL-7 (584), IL-9 (585), IL-15 (586), and IL-21 (587). In humans, inactivating mutations of IL-2R $\gamma$ lead to an X-linked form of SCID (588). Targeted deletion of $\mathrm{IL}-2 \mathrm{R} \gamma$ in mice results in drastically reduced B and T lymphocytes, and complete loss of NK cells (589). NK cell development is therefore dependent on cytokine signaling through the common gamma receptor. The NSG model has greater immunodeficiency compared to NOD/SCID mice, as originally demonstrated by superior engraftment of human hematopoietic stem cells (581); an additional benefit is that NSG mice do not develop spontaneous thymic lymphomas. As would be expected, NSG mice are deficient in NK cells. In the normal immune system, NK cells have cytotoxic activity against tumor cells, and NK cell function represents an important barrier to tumor cell proliferation and metastasis $(590,591)$. The metastatic capability of cancer cells depends greatly on the model in which they are tested (576), and NSG mice represent a useful model system to study spontaneous metastasis of cancer cells. In NSG mice, 
MDA-MB-231 cells readily form macro-metastases in the axillary lymph nodes, lungs, and liver (Fig. 21). Metastasis to other organs is sporadic (537).

\section{Functions of KLF4 in metastasis}

Literature on the role of KLF4 in cancer metastasis is varied. In some observational reports, expression of KLF4 is correlated with metastasis. For example, nuclear expression of KLF4 protein as determined by immunohistochemistry was associated with metastasis of squamous cell carcinoma (SCC) in human patients (592). Interestingly, nuclear KLF4 was also associated with moderate and poor tumor differentiation. This finding is similar to a relationship observed in breast cancer, in which increased KLF4 nuclear staining was associated with higher histologic grade and poorer prognosis in patients with early-stage disease (477).

KLF4 is also implicated in breast cancer metastasis to the brain (483). miR-7 was found to directly target KLF4, and overexpression of miR-7 suppressed KLF4 levels and reduced brain metastasis in an animal model. In contrast, bone metastasis was not reduced in association with miR-7 expression. In a set of human brain metastasis specimens, miR-7 was decreased and KLF4 was increased as compared to the primary breast tumors (Fig. 22). In this report, KLF4 expression was not functionally assessed in relation to metastasis. Additionally, suppression of KLF4 by miR-29a may be associated with metastasis and poor prognosis in colorectal cancer (593). NumbI-mediated suppression of KLF4-dependent transcription is similarly implicated in the metastasis of lung cancer cells (594). 
Functional studies of KLF4 in metastasis have employed spontaneous metastasis assays. The function of KLF4 in the metastasis of autochthonous tumors is unclear, although KLF4 expression in the stromal compartment may contribute to the spontaneous metastasis of breast cancer cells (595). Ectopic expression of KLF4 results in reduced metastasis in models of gastrointestinal malignancy, including pancreatic (471), colorectal (596), and hepatocellular (597) cancers. This finding is also reported in breast cancer (481). Overexpression of KLF4 can strongly suppress proliferation in vitro, and may similarly slow the growth of primary tumors. It is important to consider these effects when interpreting the results of experiments involving exogenous KLF4 and metastasis. Depending on the mechanism under study, re-expression of lost KLF4 to physiologic levels may enable normal course of tumor development, thereby clarifying the effect of KLF4 on metastasis (596). In a breast cancer model using murine 4T1 cells, the activity of KLF4, as affected by tamoxifen treatment of cells expressing a KLF4-ER fusion protein, seemed to have a negative effect on metastasis (380). The actual determination of metastasis was reported as the "metastatic index", in which bioluminescence-determined lung and liver metastasis is normalized to primary tumor weight.

There is at least one report of the function of endogenous KLF4 in breast cancer metastasis (598). In this study, silencing of KLF4 in murine 4T1 cells led to decreased spontaneous formation of pulmonary nodules. Knockdown of KLF4 in this context was associated with decreased primary tumor growth, showing a difference of $50 \%$ or more in tumor volumes across time points. The effect of KLF4 on spontaneous metastasis could thus be a consequence of the difference in primary tumor volume. 
The functions of KLF4 in metastasis are likely not limited to tumor cells alone. While $K l f 4^{-/-}$mice are born alive but quickly perish (388), this can be overcome through the use of tissue-specific knockout, or through an inducible system such a Cre-ER Klff ${ }^{\text {floxflox }}$ mouse model (595). Using inducible and spontaneous Klf4 knockout systems, mice had decreased KLF4 expression in the bone marrow. After intravenous injection of murine melanoma or breast cancer cells, the mice with KLF4 knockout developed fewer lung metastases, with fewer accompanying myeloid-derived suppressor cells (MDSCs). Further experiments showed that the decrease in metastasis was attributable to KLF4 knockout in the MDSCs, which led to fewer MDSC-derived fibrocytes and myofibroblasts. These data demonstrate a pro-metastatic effect of KLF4 expression in the microenvironment of cancer metastases.

\section{Questions addressed in this dissertation}

Aberrant signaling events are intrinsic to the pathogenesis of cancer, and transcription factors influence all aspects of malignancy. Cancer cells exhibit widespread phenotypic abnormalities, and the molecular pathways involved in malignancy present opportunities for therapeutic intervention. The dual topics of this dissertation are GLI1 and KLF4, zinc finger transcription factors that exemplify the subversion of normal biological mechanisms in cancer.

The Hedgehog signal pathway is a fundamental determinant of animal development, and is implicated in cancer. GLI1 is a potent transcriptional activator and effector protein of the Hedgehog signaling pathway. In normal and malignant cells, GLI family proteins are critically regulated by the $\mathrm{E} 3$ ubiquitin ligase $\mathrm{SCF}^{\beta-T r C P}$. Hedgehog-GLI signaling is implicated in cancer of the exocrine pancreas, yet the cause of Hedgehog pathway activation in this setting is unclear. 
This dissertation presents a novel mechanism of GLI1 protein stabilization involving the HMGbox transcription factor SOX9. In addition, this research addresses the functional significance of SOX9 towards GLI1 activity in pancreatic cancer cells.

Similarly, the transcription factor KLF4 is important in numerous pathogenic and nonpathogenic contexts. Although the functions of KLF4 in some malignancies are wellcharacterized, the contribution of KLF4 to breast cancer is less certain. In particular, the function of KLF4 in breast cancer metastasis is poorly defined. Research presented in this dissertation documents the functional role of KLF4 in the metastasis of breast cancer cells. We address the effects of KLF4 expression on the growth of orthotopic mammary tumors, circulating tumor cells, and the spontaneous development of lung and liver metastases. We also examine the relationship of KLF4 and DDR1, a collagen-binding RTK that may influence the antimetastatic activity of KLF4. 


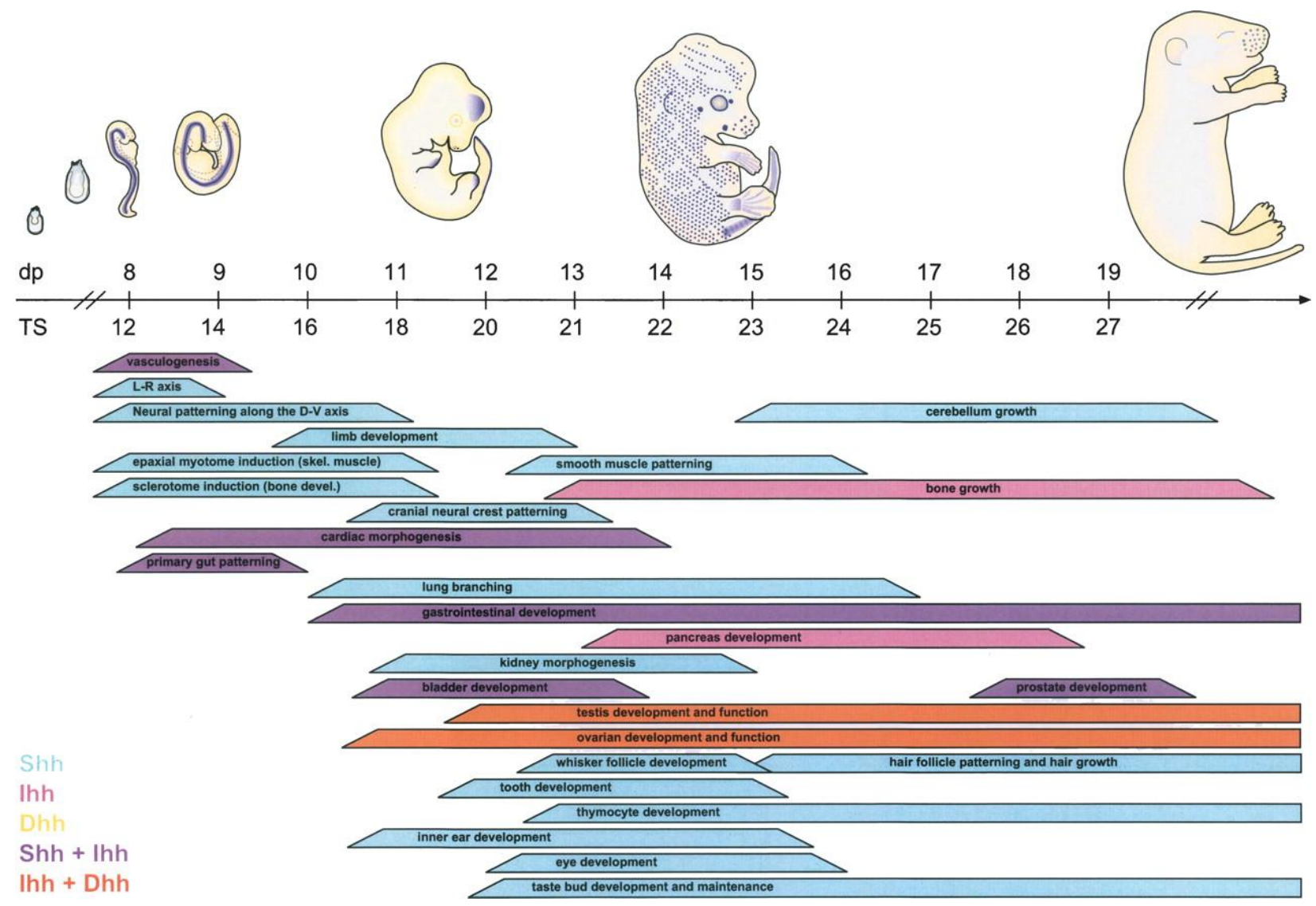

Figure 1. Hedgehog signaling controls mouse development through Shh, Ihh, and Dhh. From Varjosalo, M and Taipale, J Genes Dev 2008 (2). Used under license (CC-BY-NC 4.0). The timeline of embryonic stages is indicated by days post coitum (dp) and Theiler stage (TS). Expression of Ptch, a Hh target gene, is shown as blue shading in the embryonic representations above the timeline. Bars below show when various developmental processes are controlled by Hh ligands, alone or in combinations according to the color scheme at lower left. 

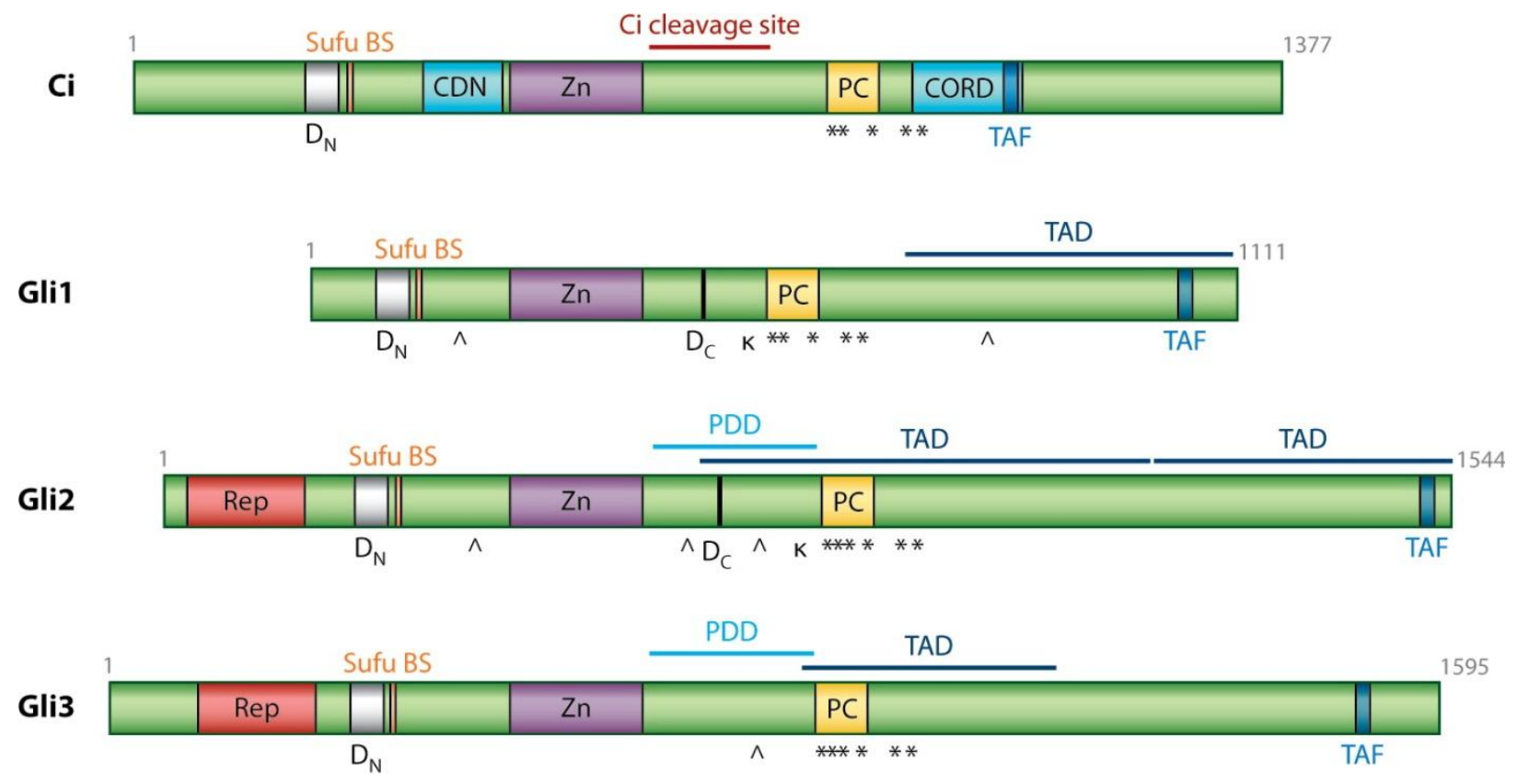

\begin{tabular}{|c|c|c|c|}
\hline $\mathrm{Zn}$ & $\begin{array}{l}\text { Zinc-finger DNA- } \\
\text { binding domain }\end{array}$ & PC & $\begin{array}{l}\text { Protein Kinase A site } \\
\text { Phosphorylation cluster }\end{array}$ \\
\hline TAD & $\begin{array}{l}\text { Transcription activation } \\
\text { domain }\end{array}$ & PDD & $\begin{array}{l}\text { Processing determinant } \\
\text { domain }\end{array}$ \\
\hline TAF & $\begin{array}{l}\text { TAF-binding site } \\
\text { acidic activation motif }\end{array}$ & $D_{N^{\prime}} D_{C}$ & Degrons \\
\hline Rep & Repressor domain & $\wedge$ & Sumoylation site \\
\hline CDN, CORD & Cos2-binding sites & $\mathrm{K}$ & Acetylation site \\
\hline Sufu BS & Sufu-binding site & & \\
\hline
\end{tabular}

Figure 2. GLI proteins are effectors of Hedgehog signaling. From Hui, CC and Angers, S Annu Rev Cell Dev Biol 2011 (37). Permission allowed for dissertation use. Schematics indicate features of Drosophila melanogaster Cubitus interruptus protein, and Mus Musculus GLI1, GLI2, and GLI3 proteins. Note the absence of the Repressor and Processing determinant domains in GLI1. For comparison, human GLI1 contains 1107 amino acid residues. 

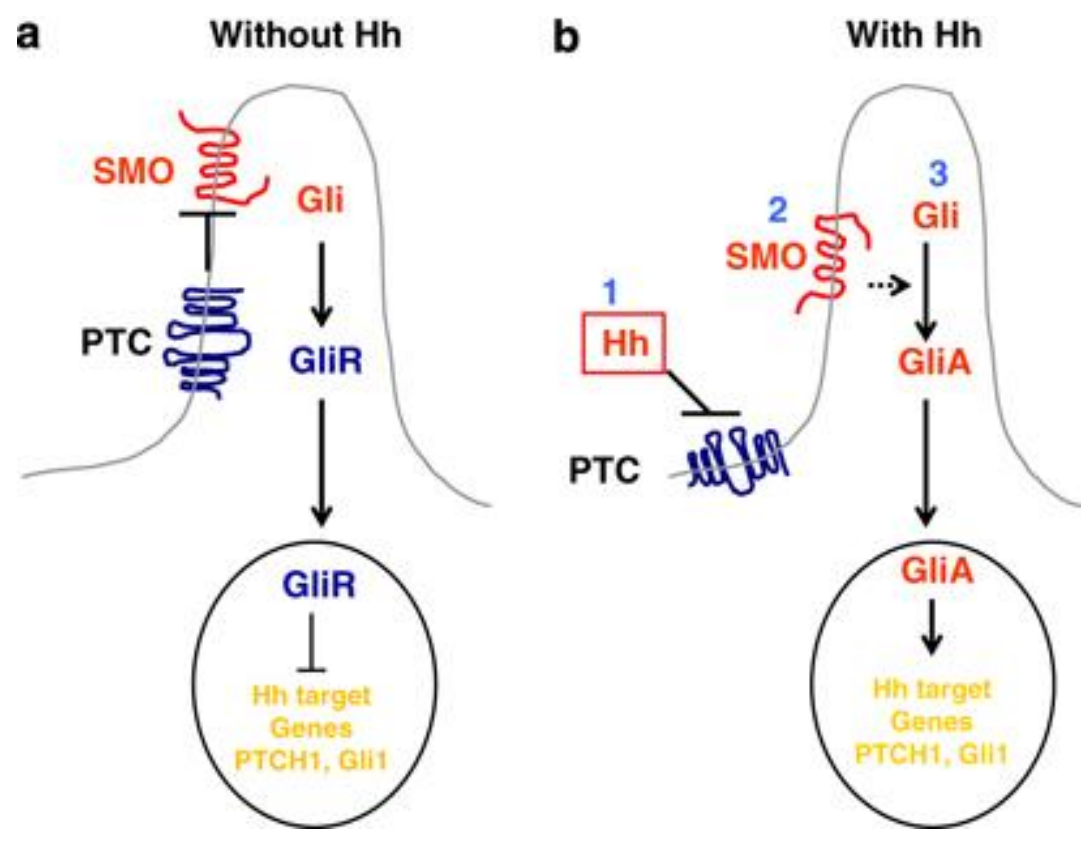

Figure 3. A simplified schematic of Hedgehog signaling in vertebrates. Used with permission from Yang, L et al. Oncogene 2010 (94). (A) In the absence of Hh ligand, the receptor Patched (Ptch/Ptc) functions to inhibit the G protein-coupled receptor Smoothened (Smo). The GLI family of transcription factors are processed or degraded such that GLI acts as a transcriptional repressor $\left(\mathrm{GLI}^{\mathrm{R}}\right)$ of Hh target genes. (B) Hh ligands bind Patched, which alleviates Patchedmediated inhibition of Smoothened. This alters the processing and degradation of GLI proteins, creating GLI forms that activate transcription $\left(\mathrm{GLI}^{\mathrm{A}}\right)$ of Hh target genes. In vertebrates, many events in Hh signaling occur at the primary cilia. The mechanisms controlling the processing and degradation of GLI proteins are not shown, but include phosphorylation by kinases including PKA, GSK3 $\beta$, and CK1, and interaction with the ubiquitin-proteasome system through the E3 ligase $\mathrm{SCF}^{\beta-\operatorname{TrCP}}$. Effects on Smo localization, and interaction of key regulators such as Sufu, are also not shown. Examples of Hh target genes include PTCHI and GLII, which create negative and positive feedback, respectively. 

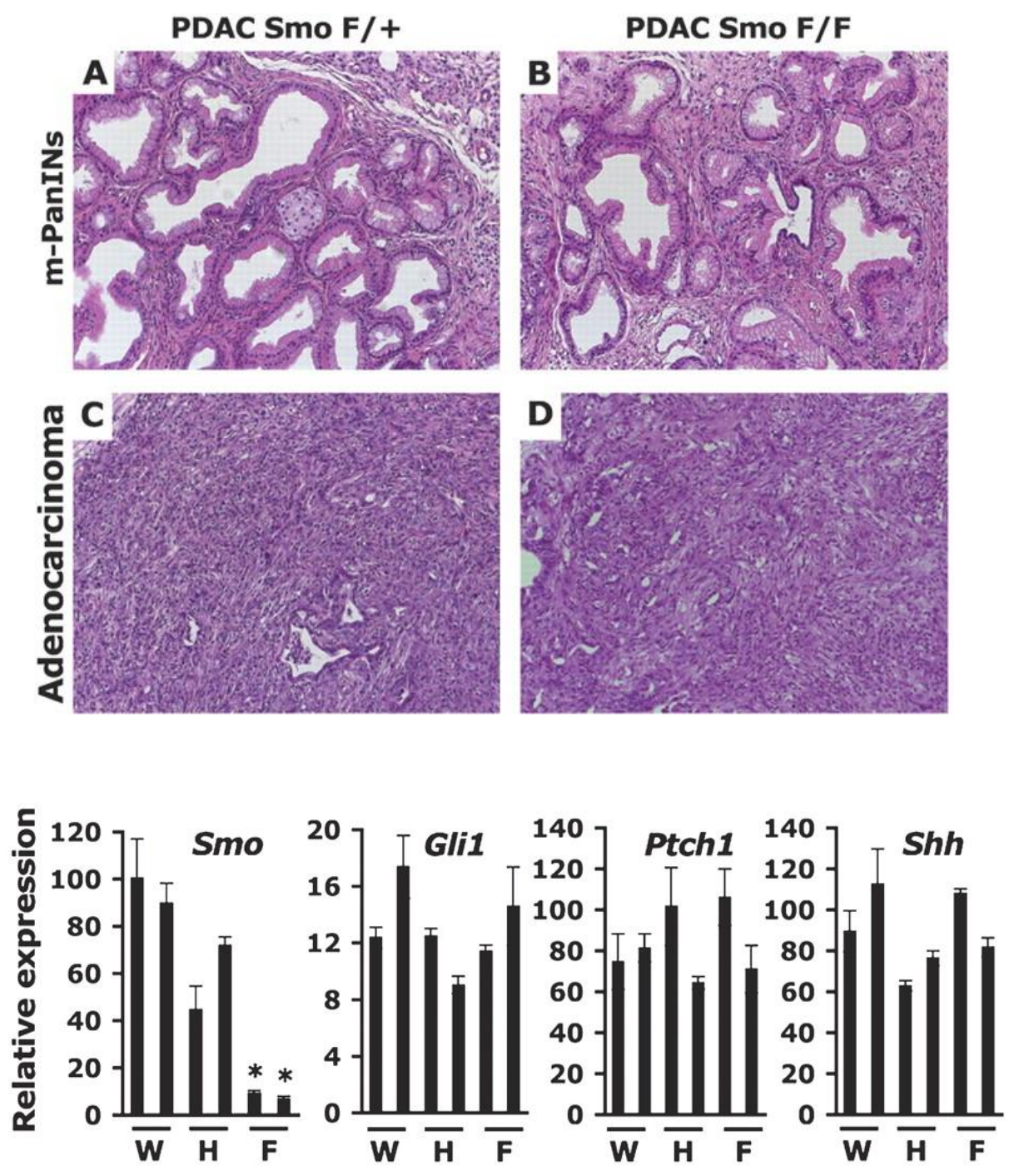

Figure 4. Smoothened-independence of Hedgehog signaling in Kras-driven pancreatic ductal adenocarcioma. Adapted from Nolan-Stevaux, O et al. Genes Dev 2009 (34). Used under license (CC-BY-NC 4.0). Upper panels (A-D): Deletion of Smo in the pancreatic epithelium does not inhibit development of pancreatic intraepithelial neoplasia (A,B) or PDA (C,D) in PtflaCre;LSL-Kras ${ }^{G 12 D} ; \operatorname{Trp} 5 f^{f l o x / w t}$ mice. In fact, Smo ${ }^{F / F}$ mice on average had slightly worse survival in comparison to $S m o^{F /+}$ mice in this model of PDA (not shown). In a prior study, phenotypic defects were not observed in mice heterozygous for a Smo null allele (23). Lower panel: Expression of Hh target genes Glil and Ptchl is maintained in Kras-driven PDA, regardless of 
Smo depletion. PDA lesions were microdissected from two mice each of $\operatorname{Smo}^{+/+}(\mathrm{W}), \mathrm{Smo}^{\mathrm{F} /+}$ $(\mathrm{H})$, and $S m o^{F / F}(\mathrm{~F})$ groups. Transcription of Hh target genes GLII and PTCHI is a wellcharacterized indicator of GLI protein transcriptional activity $(39,85)$. The maintenance of Gli1 and Ptchl mRNA levels in the absence of functional Smo is therefore indicative of noncanonical Hh signaling, independent of upstream factors such as hedgehog ligands, Ptch, and Smo. The expression of Shh by PDA cells is thought to exert paracrine signaling effects on stromal cells such as cancer-associated fibroblasts. 


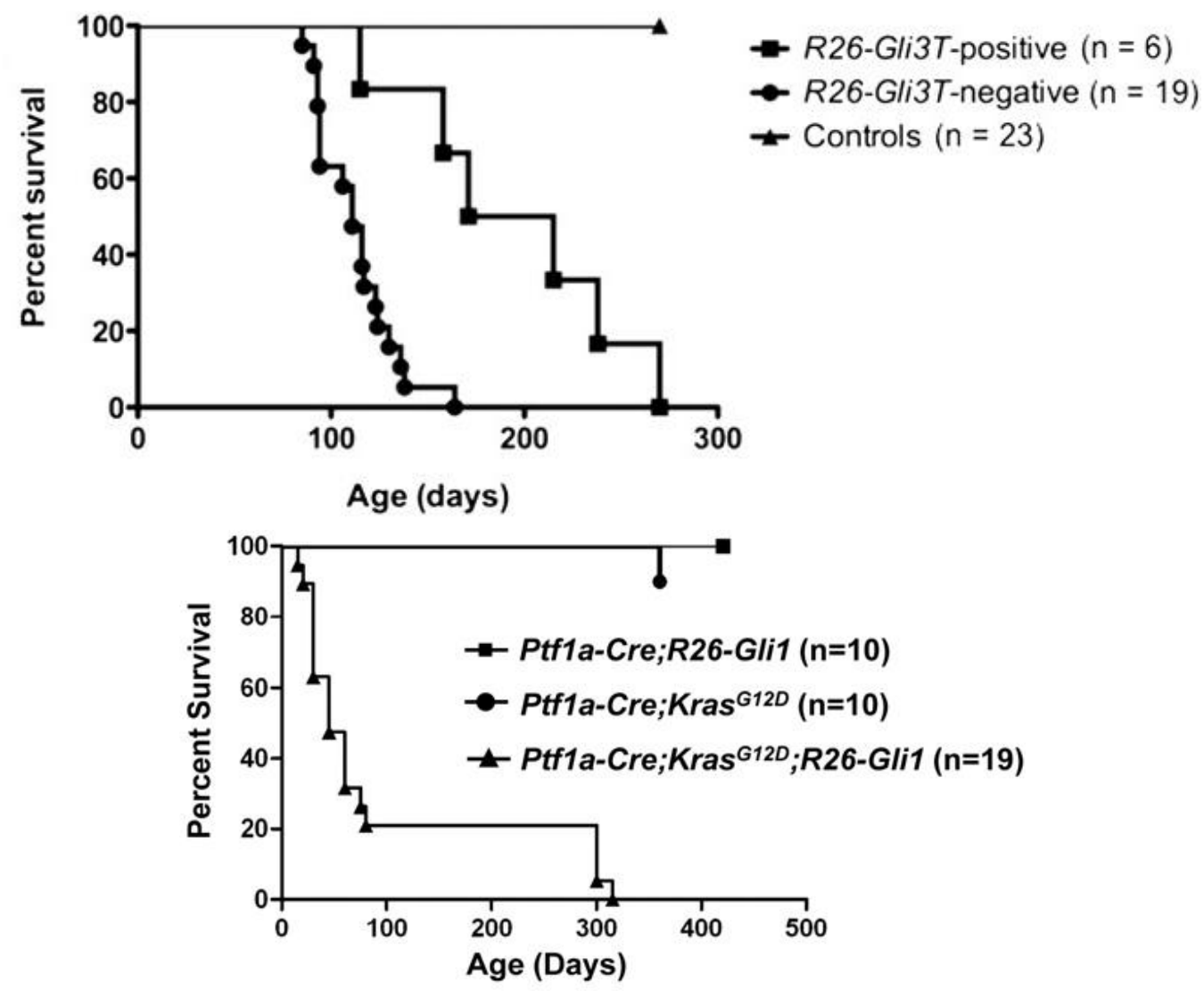

Figure 5. Role of GLI transcriptional activity in Kras-induced pancreatic ductal adenocarcinoma. From Rajurkar, M et al. Proc Natl Acad Sci U S A 2012 (130). Permission allowed for noncommercial use. Upper panel: Effect of suppressing GLI activity in a Kras-driven mouse model of PDA. The development of PDA in Ptfla-Cre;LSL-Kras ${ }^{G 12 D}$;Trp $53^{\text {flox/wt }}$ mice is delayed through expression of Gli3T (R26-Gli3T), which suppresses GLI transcriptional activation. Lower panel: GLI1 expression accelerates development of Kras-driven PDA. Ectopic GLI1 expression alone (Ptfla-Cre;R26-Gli1) did not affect pancreatic development (not shown) or initiate tumors. The slow development and progression of PanIN and PDA from activated Kras $\left(\right.$ Ptfla-Cre; $\left.\mathrm{Kras}^{G 12 D}\right)$ is greatly accelerated by ectopic GLI1 (Ptfla-Cre;Kras ${ }^{G 12 D} ;$ R26-Gli1). Note that $\operatorname{Trp} 53$ is not manipulated in this model, in contrast to the experiment presented in the upper panel. 


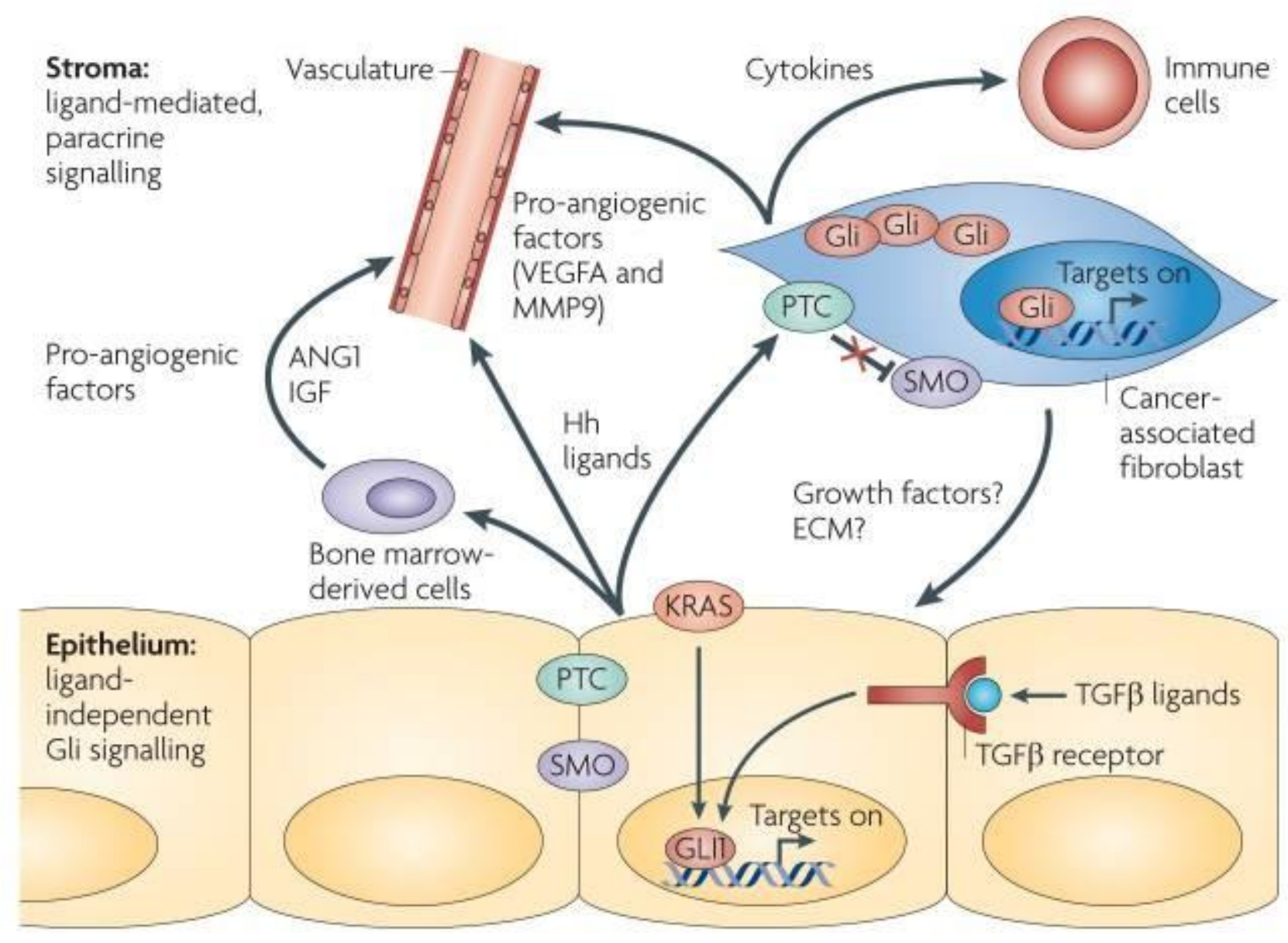

Figure 6. Hedgehog signaling in pancreatic ductal adenocarcinoma. Used with permission from Morris, JP et al. Nat Rev Cancer 2010 (132). In the stroma, Hh ligands expressed from carcinoma cells signal to cell types such as cancer-associated fibroblasts. This paracrine, liganddependent mechanism can alter the tumor microenvironment through effects on the extracellular matrix, angiogenesis, immune response, growth factor signaling, and other pathways. In contrast, Hh signaling is activated in tumor cells through non-canonical mechanisms, including through KRAS and TGF $\beta$ signaling. ANGPT1, angiopoietin 1; IGF, insulin-like growth factor; MMP9, matrix metalloproteinase 9; VEGFA, vascular endothelial growth factor A. 


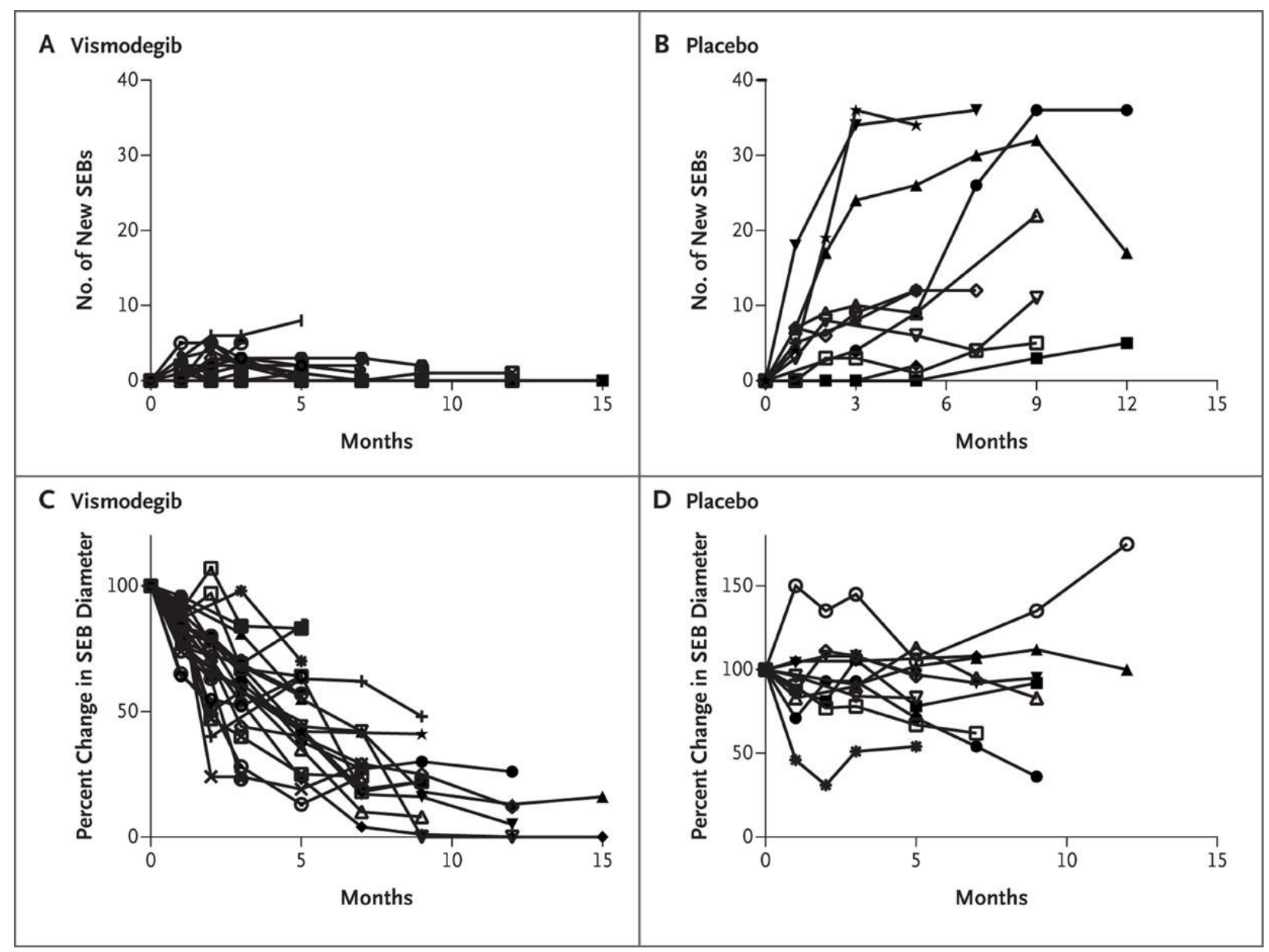

Figure 7. Therapeutic benefit of the Hedgehog pathway inhibitor vismodegib in a randomized, double-blind, placebo-controlled trial in patients with nevoid basal cell carcinoma syndrome. From Tang, JY et al. N Engl J Med 2012 (191). Permission allowed for dissertation use: Reproduced with permission from (191) Copyright Massachusetts Medical Society. (A,B) Number of new surgically eligible basal cell carcinomas (SEBs) in patients receiving vismodegib (A) or placebo (B). (C,D) Change in existing SEB size in patients receiving vismodegib (C) or placebo (D), as shown by the percent change from baseline in the sums of the longest diameters of SEBs. 


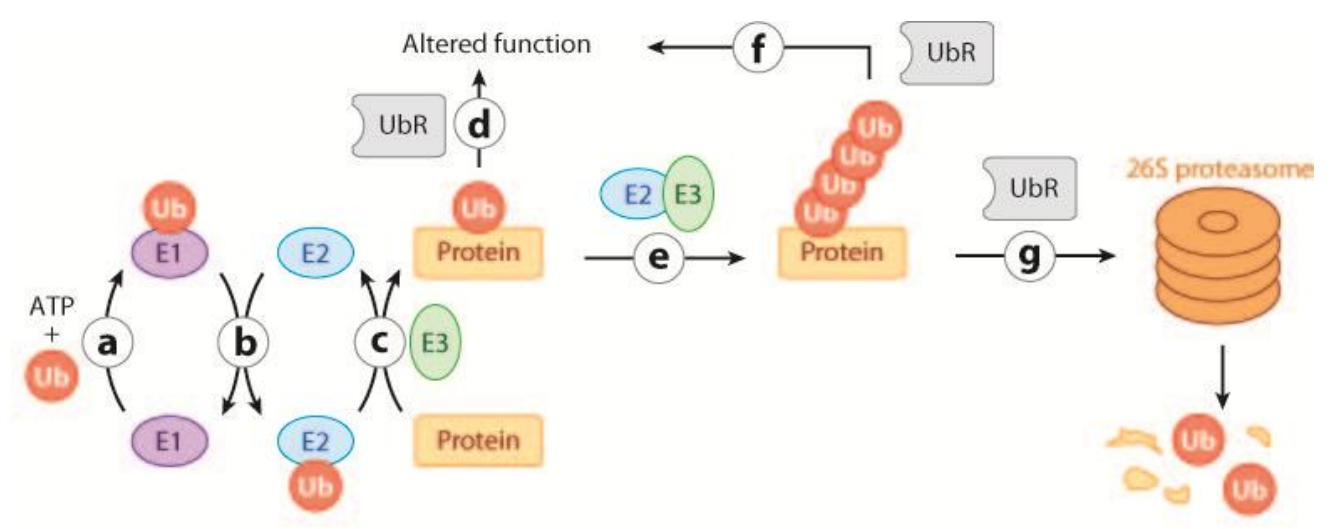

Figure 8. The ubiquitin system. Used with permission from Deshaies, RJ and Joazeiro, CA Annu Rev Biochem 2009 (244). (A) E1 ubiquitin-activating enzyme activates ubiquitin and ubiquitinlike proteins, so they may be transferred to an E2 ubiquitin-conjugating enzyme. (B) Ubiquitin is conjugated to a cysteine residue of an E2 enzyme through a thioester link. (C) An E3 ubiquitin ligase catalyzes the transfer of ubiquitin from the E2 ubiquitin conjugating enzyme to the protein substrate. (D) Monoubiquitylated proteins can dissociate from the ubiquitin ligase complex. The monoubiquitin then alters protein function through effects on protein interactions or other mechanisms. (E) Monoubiquitylated substrates can also receive additional ubiquitylations, which can occur through multiple attachment of single units (not shown) or as chain of ubiquitin. (F) Polyubiquitylation forms such as K63 chains can also act to alter protein function. (G) Chains through other ubiquitin residues, including K48 and K29, direct the substrate to the proteasome for degradation or processing. Ubiquitin receptor proteins (UbRs) can determine the effects of ubiquitylation, and are important in some cases to help deliver polyubiquitylated substrates to the proteasome. 


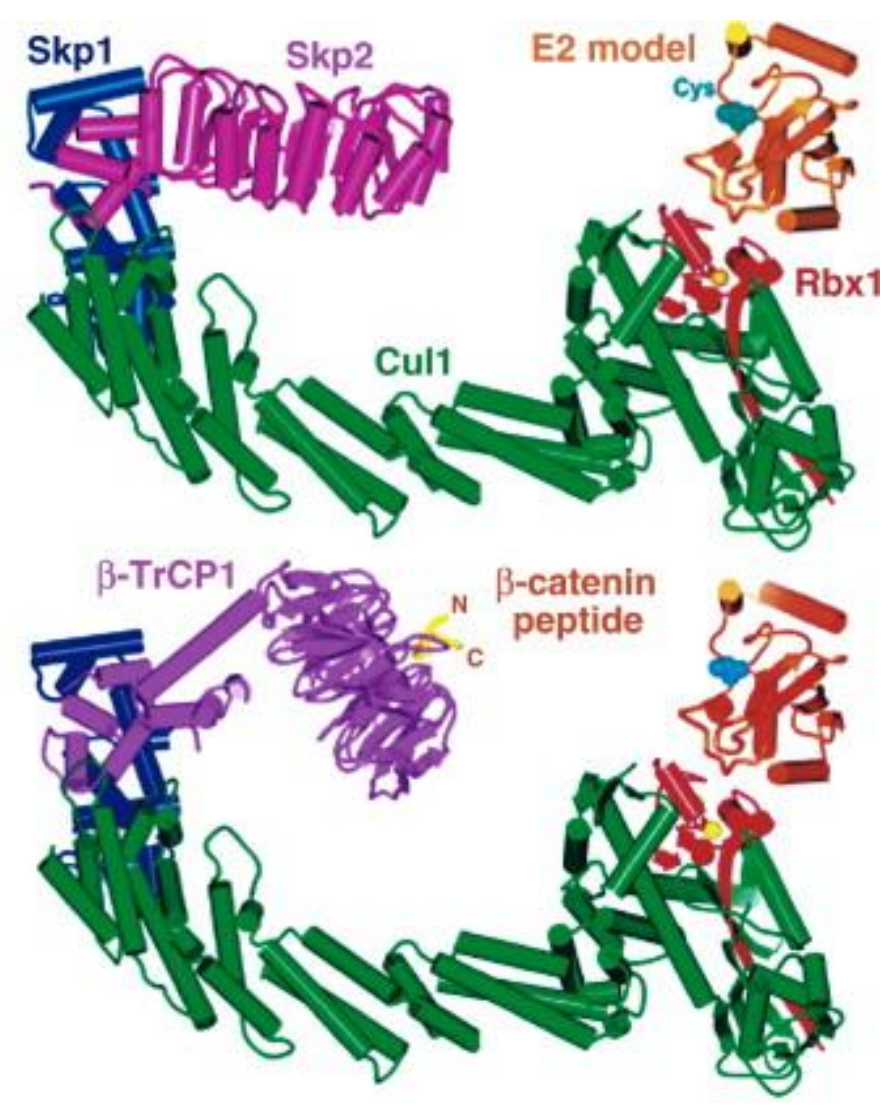

Figure 9. Structure of F-box containing E3 ubiquitin ligases. Used with permission from $\mathrm{Wu}, \mathrm{G}$ et al. Mol Cell 2003 (282). Two related E3 ubiquitin ligase structures are shown: SCF ${ }^{\mathrm{SKP} 2}$ (topmost complex), and $\mathrm{SCF}^{\beta-\operatorname{TrCP}}$ (lower complex). Rbx1 is included in both structures. The position of the $\mathrm{E} 2$ conjugating enzyme $\mathrm{UbcH} 7$, which interacts with $\mathrm{Rbx} 1$, and the active cysteine residue, which conjugates ubiquitin, are indicated. A substrate peptide fragment of $\beta$ catenin is shown in association with the WD repeat motif of $\beta$ - TrCP. The torus-like WD repeat structure is viewed from the side. The E3 ligase catalyzes transfer of ubiquitin from the E2 enzyme to the substrate. 


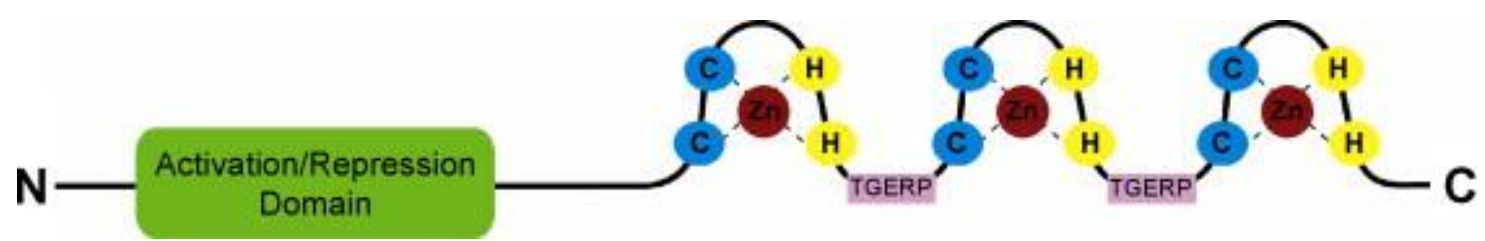

Figure 10. Overview of KLF structure. Used with permission from Pearson, R et al. Int J Biochem Cell Biol 2008 (328). KLFs contain three $\mathrm{C}_{2} \mathrm{H}_{2}$ zinc fingers. The linking region between the KLF zinc fingers is also highly conserved. Other elements of KLFs are poorly conserved, but usually contain at least one domain that functions in transcriptional activation or repression. 


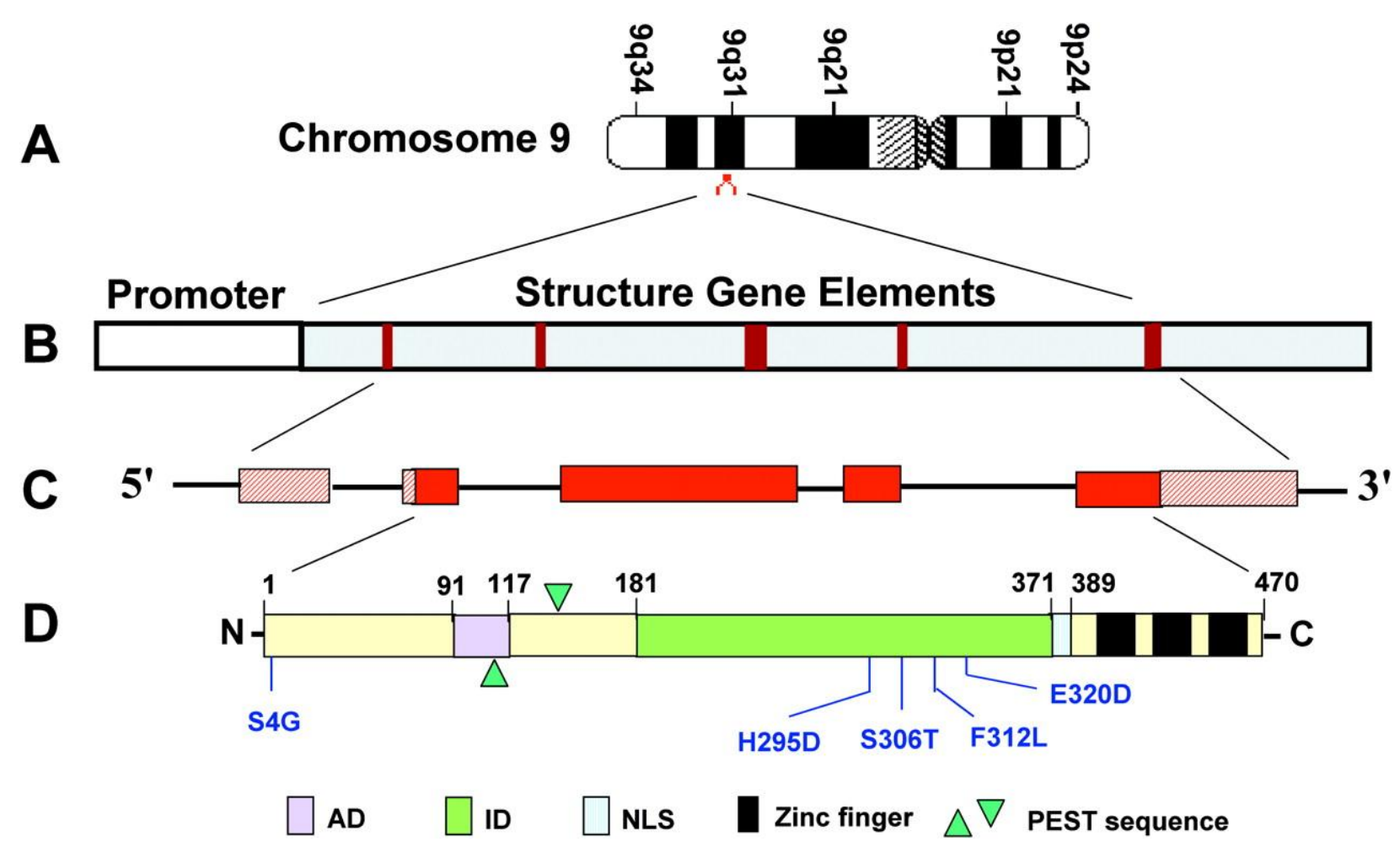

Figure 11. Human KLF4 locus, mRNA, and protein structure. Used with permission from Wei D et al. Carcinogenesis 2006 (599). (A) KLF4 spans a $6.3 \mathrm{~kb}$ region of chromosone 9q31. (B) The KLF4 gene contains five exons. (C) The KLF4 RNA transcript. Amongst the five exons, the open reading frame is found in exons 2-4, indicated by solid red. (D) KLF4 protein contains 470 amino acid residues. Also shown are zinc fingers, a transcriptional activation domain (AD), inhibitory domain (ID), nuclear localization signal (NLS), and PEST sequences. Point mutations reported in tumor cells are indicated in blue. 

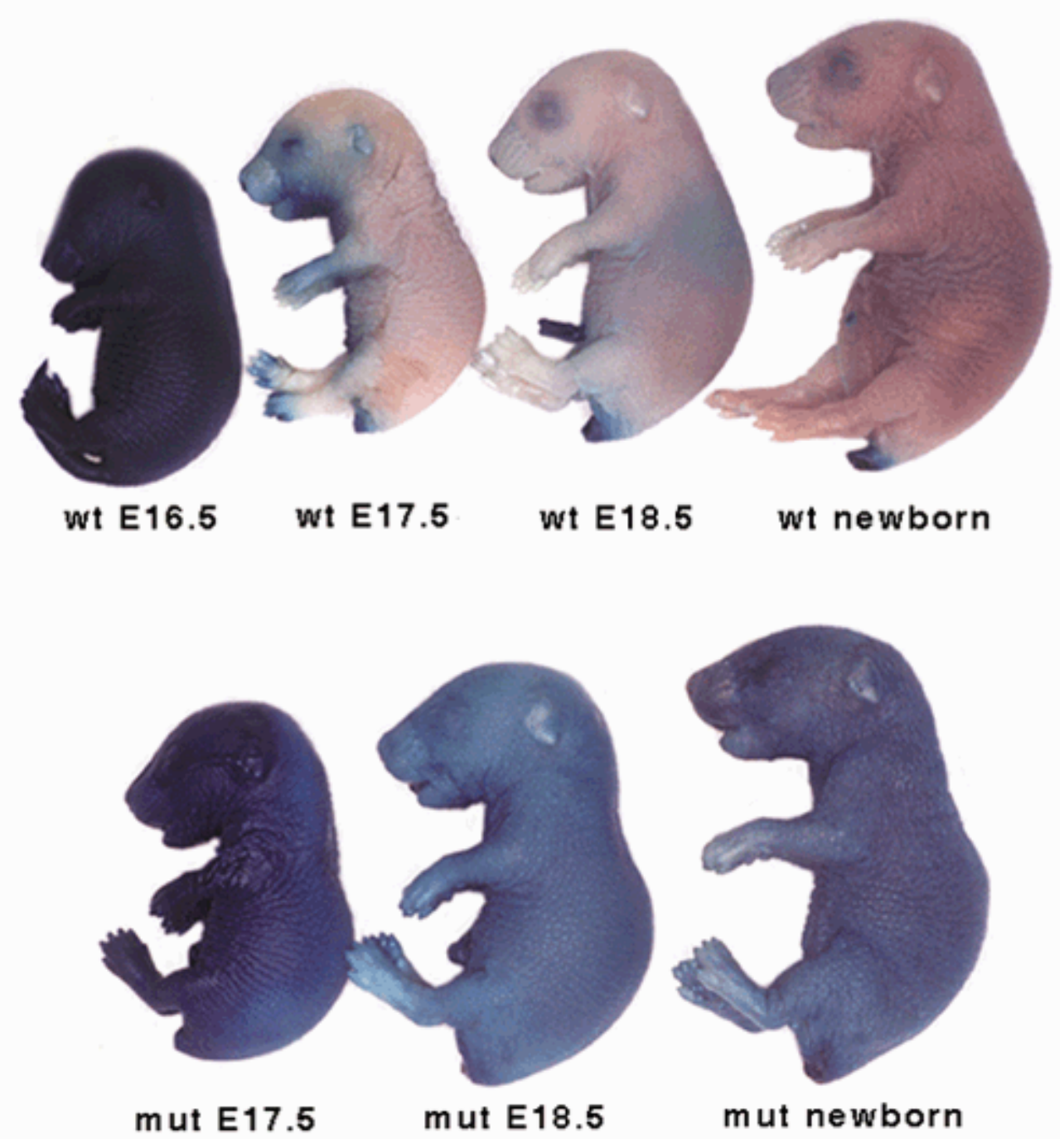

Figure 12. KLF4 is required for the skin barrier function. Used with permission from Segre, JA et al. Nat Genet 1999 (388). In normal development of the mouse embryo (upper series), the skin develops a barrier function over the last three days of gestation, which prevents absorption of external dyes. In contrast, $K l f 4^{-/}$mice do not form an effective skin barrier. In this method, X-gal that successfully penetrates the skin is cleaved by endogenous $\beta$-galactosidase activity, resulting in blue precipitates. 

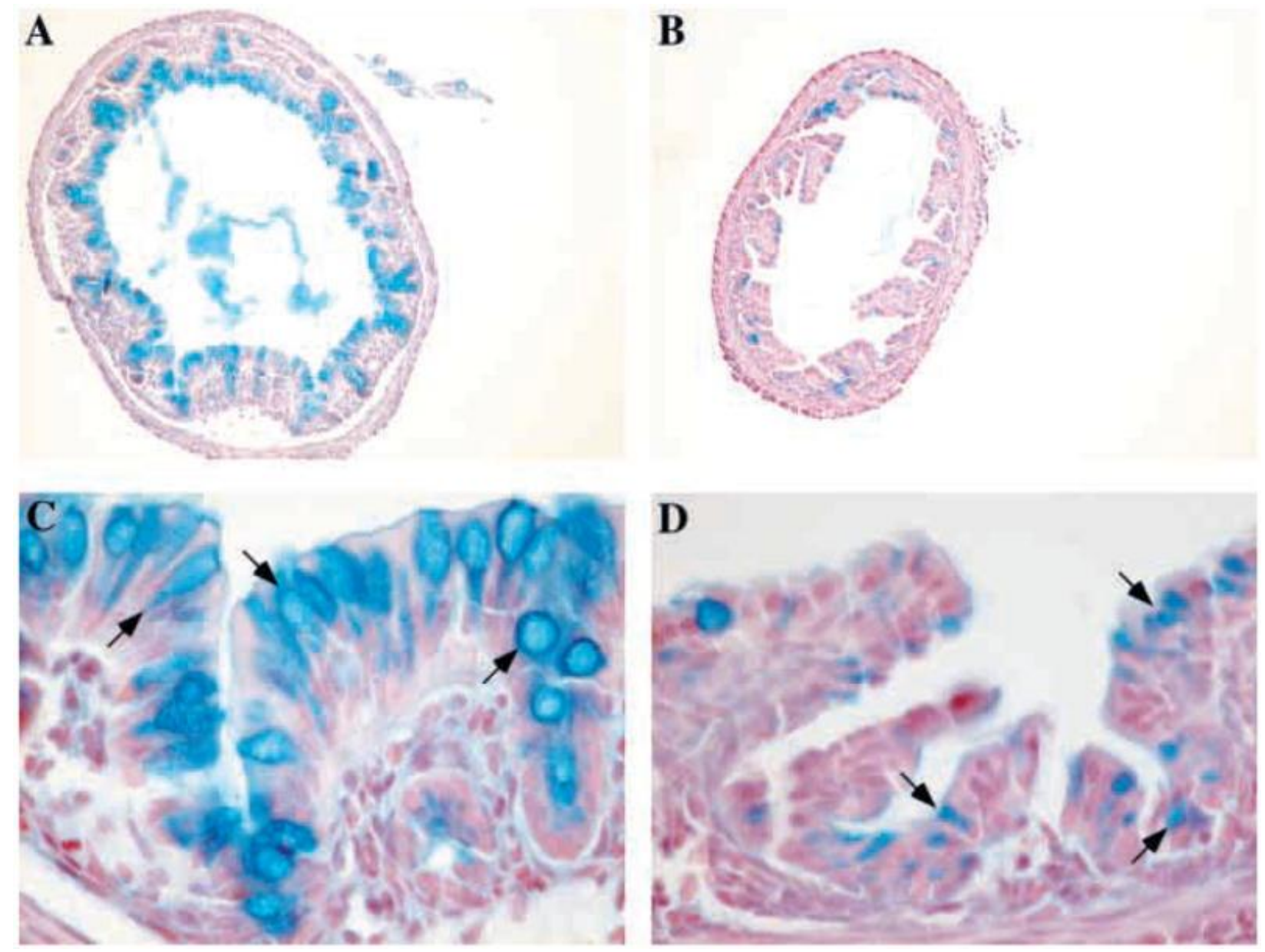

Figure 13. Goblet cell deficiency in $\mathrm{Klf4}^{-/-}$mice. Used with permission from Katz, JP et al. Development 2002 (391). In the colonic epithelium of wild-type mice (panels A and C), mature goblet cells represented $20 \%$ of all epithelial cells as determined by Alcian Blue staining and morphology (panel C arrows). In colon specimens of $\mathrm{Klf}^{-\digamma}$ mice (panels B and D), only $2 \%$ of epithelial cells were mature goblet cells. Cells with Alcian Blue staining in the Klf $4^{-/}$mouse colon did not have normal goblet cell morphology (panel D arrows). 

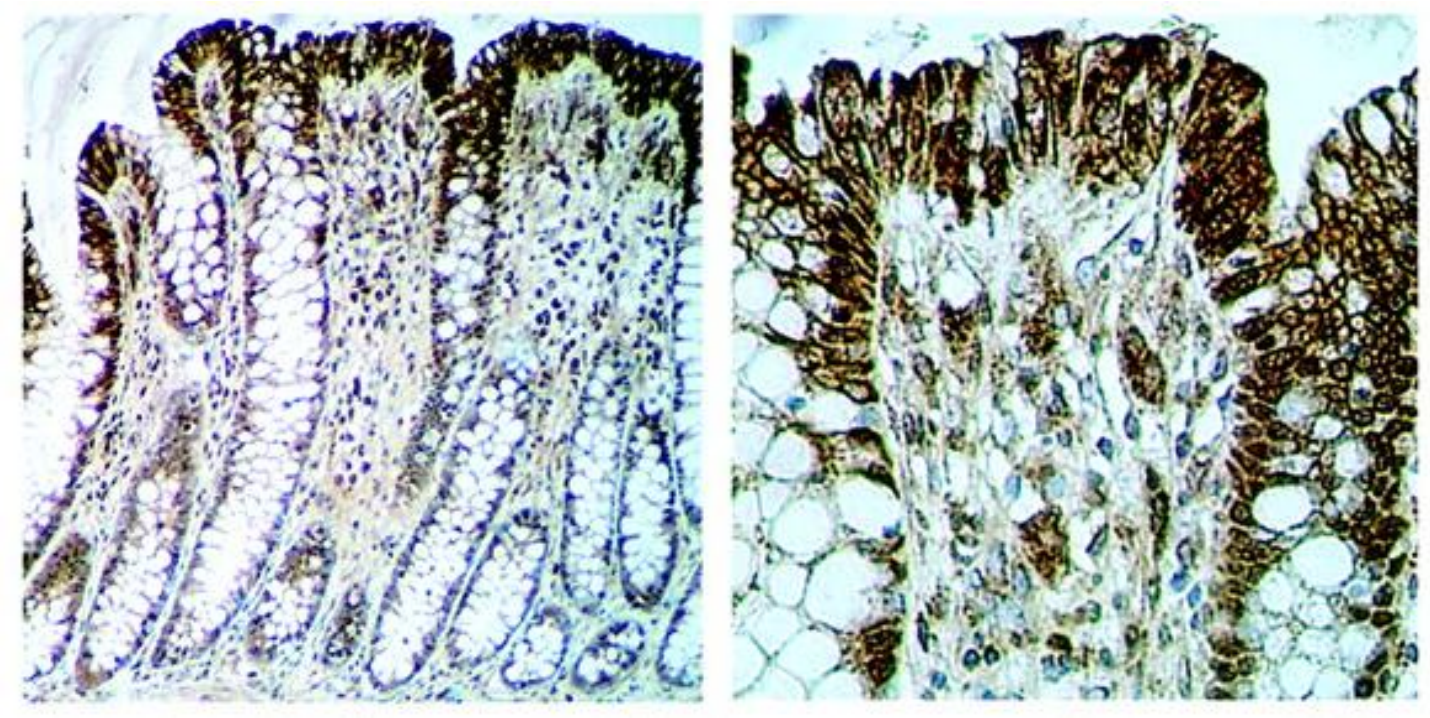

Figure 14. KLF4 expression in the normal human colonic mucosa. Adapted from Zhang, W et al. Mol Cell Biol 2006 (371). Permission allowed for dissertation use. Immunohistochemical detection of KLF4 in human colon specimens reveals a distinct pattern of expression. The base of the crypt, containing stem cells that maintain the colonic epithelium, is characterized by low expression of KLF4 protein. In contrast, expression of KLF4 protein is high in the differentiated, growth-arrested epithelial cells at the crypt apex. KLF4 expression is therefore associated with epithelial differentiation and growth arrest in the colon. 

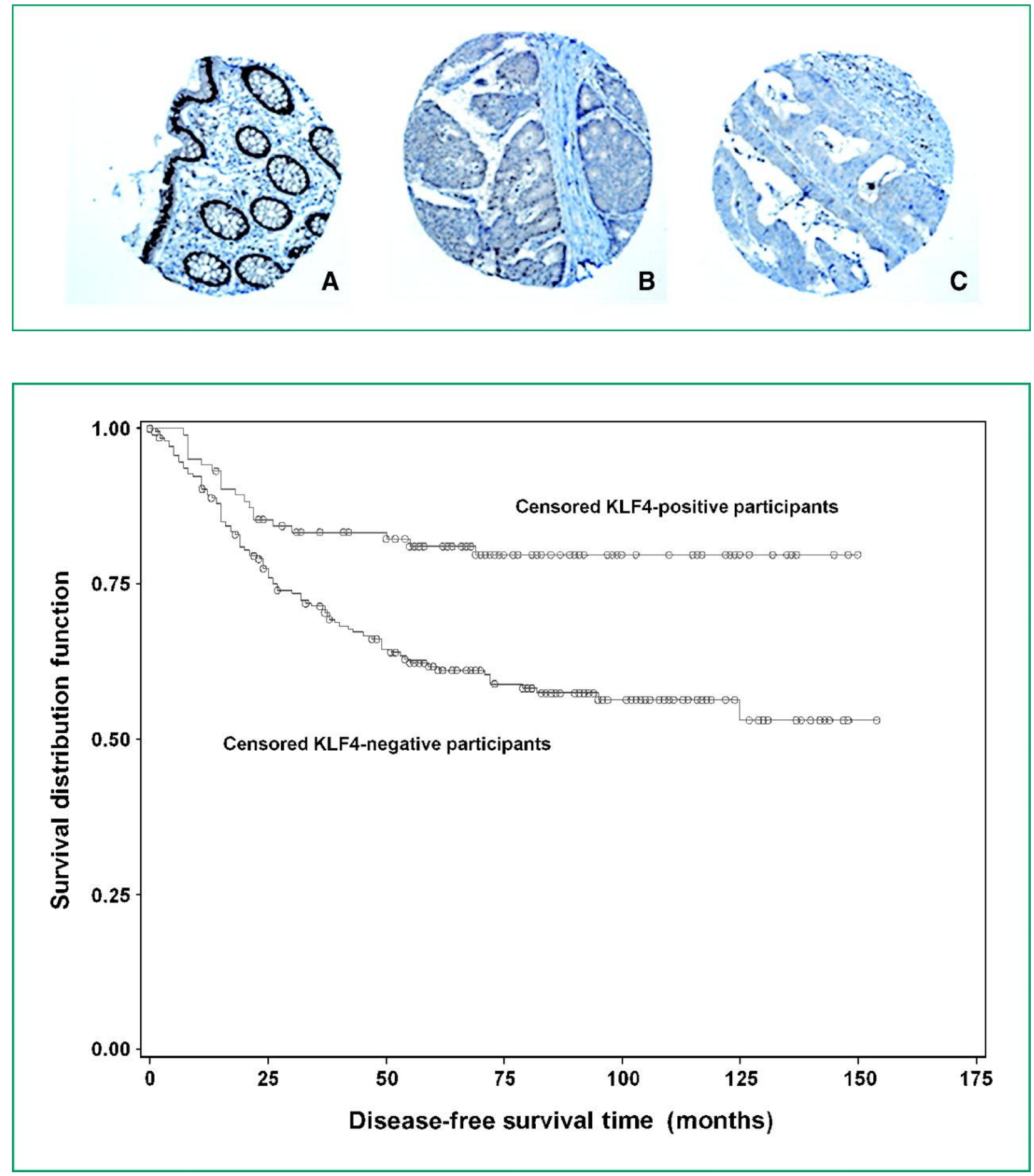

Figure 15. Association of KLF4 expression with disease-free survival in colon cancer. From Patel NV et al. Cancer Epidemiol Biomarkers Prev 2010 (458). Adapted by permission from the American Association for Cancer Research: Patel NV et al, Expression of the tumor suppressor 
Krüppel-like factor 4 as a prognostic predictor for colon cancer, Cancer Epidemiology, Biomarkers \& Prevention, 2010 October, vol 19. issue 10, 2631-8, doi: 10.1158/1055-9965.EPI10-0677. Upper panels: Expression of KLF4 protein detected by immunohistochemistry in normal human colon (A). Tumors specimens with $\geq 10 \%$ staining were scored as KLF4 positive (B), whereas tumors with less than 10\% staining were considered KLF4 negative (C). Lower panels: Disease-free survival time is significantly enhanced in patients with KLF4-expressing tumors ( $\mathrm{P}=0.0001)$. This retrospective study was performed using a tissue microarray of 367 colon cancer cores. 
Control
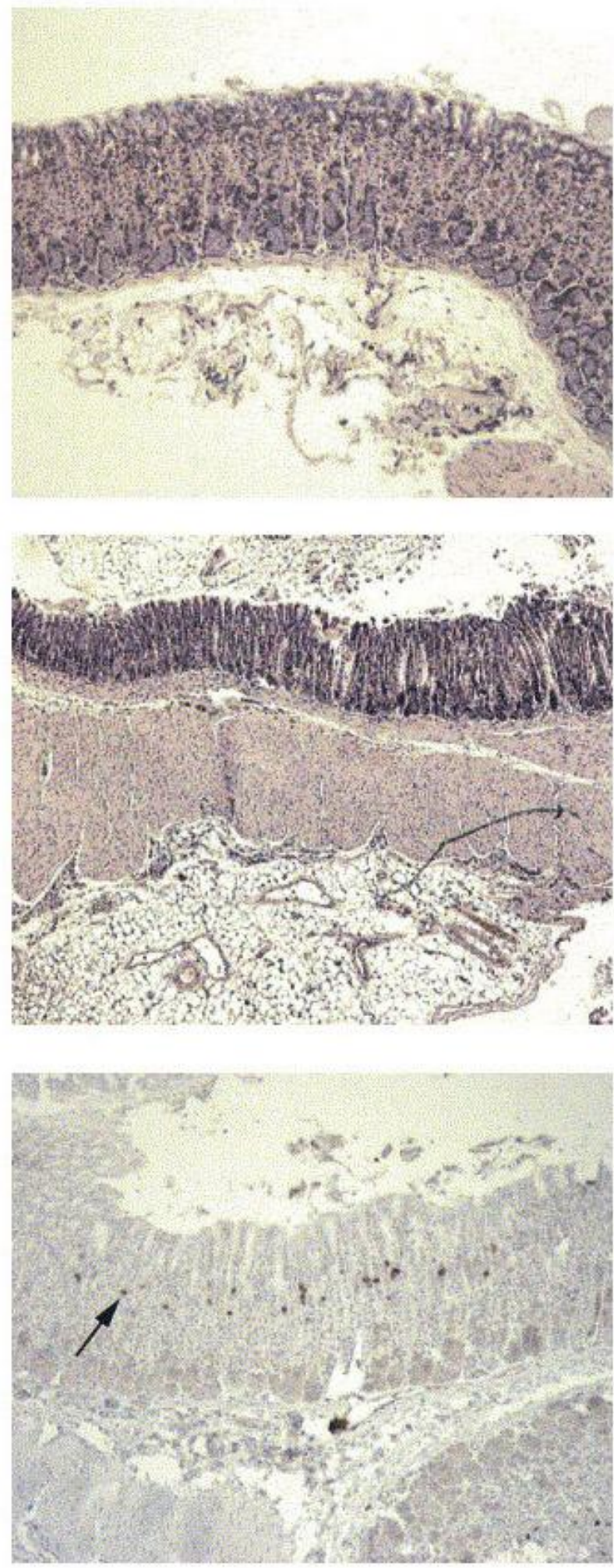

Klf4 mutant
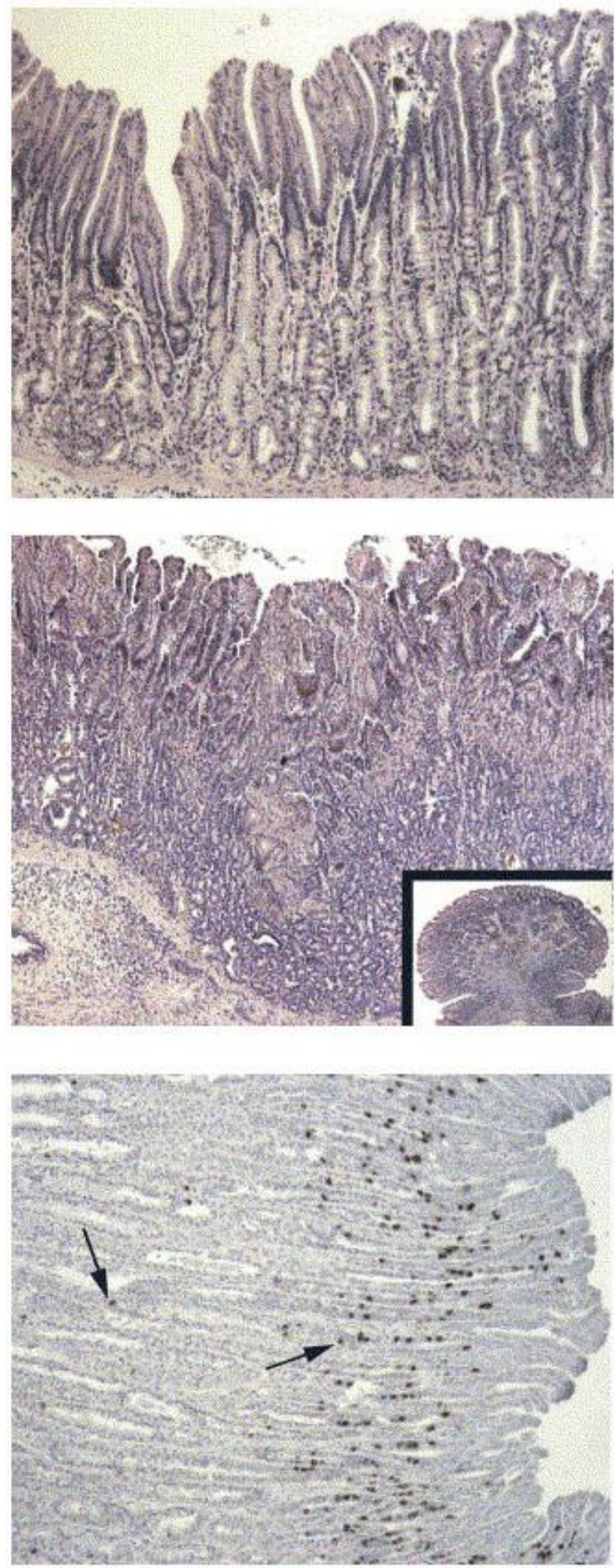
Figure 16. Tissue-specific loss of KLF4 results in precancerous changes of the gastric epithelium. Used with permission from Katz, JP et al. Gastroenterology 2005 (401). Upper panels: Compared to controls, mice with Klf4 ablation in the glandular stomach (Foxa3Cre/Klf $4^{\text {loxP/loxP }}$ ) showed epithelial hypertrophy of the epithelium and distorted gastric pit glands. There were increased mucus cells and decreased parietal cells. Middle panels: At 12 months, Klf4 loss resulted in further epithelial hypertrophy and gland distortion. Polyps were also found in Klf4 mutant mice, but gastric malignancies were not found in either group. Lower panels: Immunohistochemistry to detect bromodeoxyuridine reveals proliferating cells (arrows) in the gastric glandular epithelium. Compared to controls, there were increased numbers of proliferating cells in the Klf4 mutant epithelium, and the location of the proliferating cells was shifted towards the lumen. 

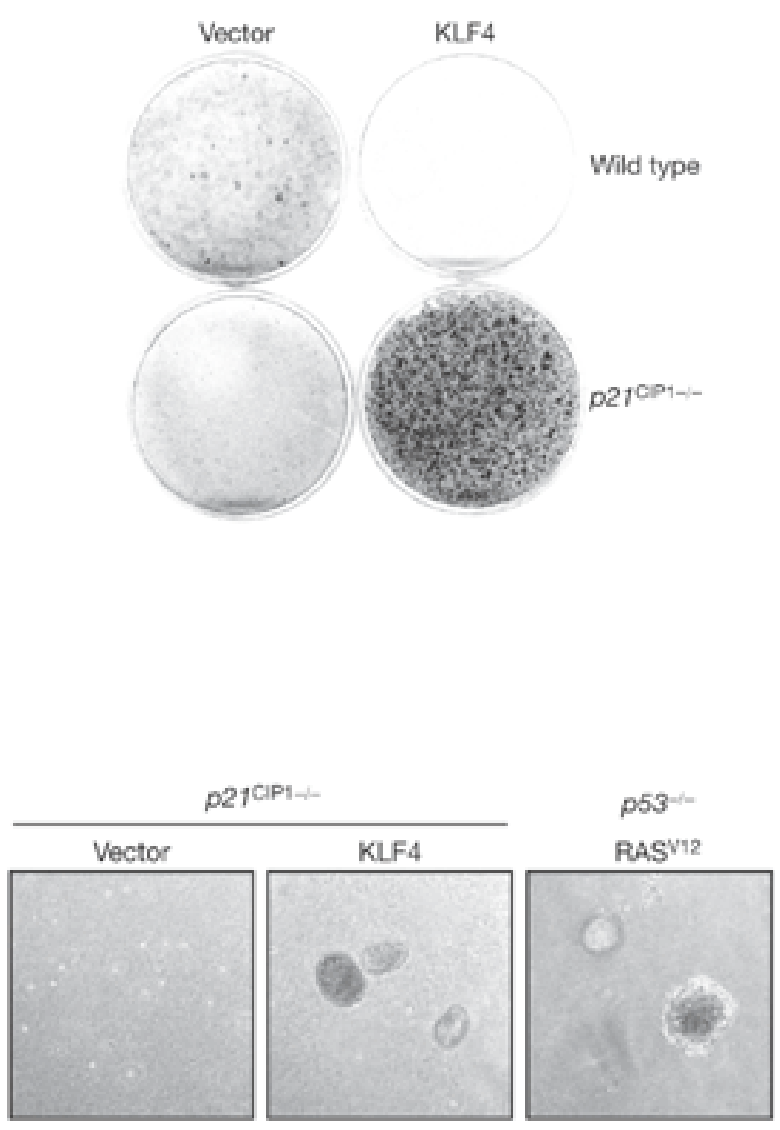

Figure 17. KLF4 promotes malignant properties in the absence of $\mathrm{p} 21$. Used with permission from Rowland, BD et al. Nat Cell Biol 2005 (440). KLF4 promotes p21 and suppresses p53, and in the absence of p21, KLF4 may exert oncogenic effects. Upper panels: In wild-type MEFs, overexpression of KLF4 lacks transforming effects. In contrast, KLF4 overexpression in p21 knockout MEFs results in proliferating colonies lacking contact inhibition. Lower panels: Overexpression of KLF4 in p21 knockout MEFs allows colony formation in soft agar, in a quantity comparable to that of $p 53^{-/}$MEFs transfected with activated RAS. 


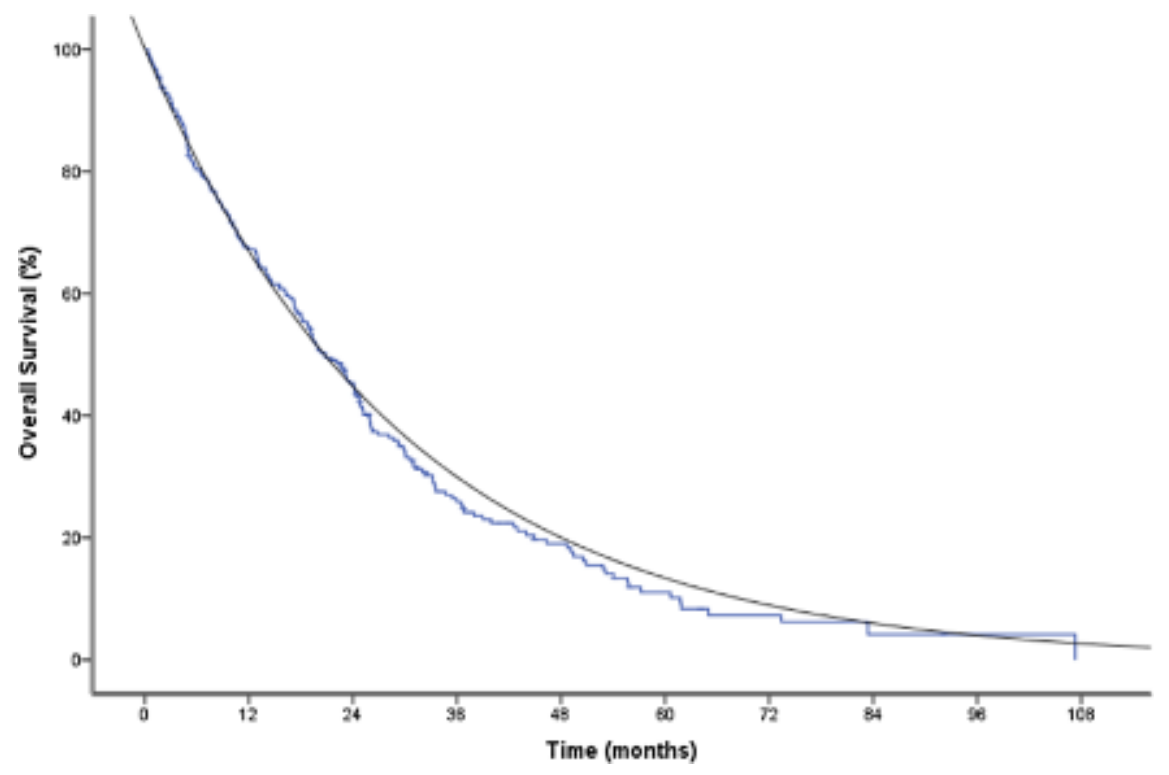

Figure 18. Survival in metastatic breast cancer. Used with permission from Thientosapol, ES et al. Intern Med J 2013 (522). The median overall survival from start of first-line chemotherapy for 273 women diagnosed with metastatic breast cancer was 20.4 months. Patients had a median age of 56 years. Tumors were hormone receptor positive in $69 \%$ of patients, HER 2 positive in $27 \%$, and triple negative in $13 \%$. 


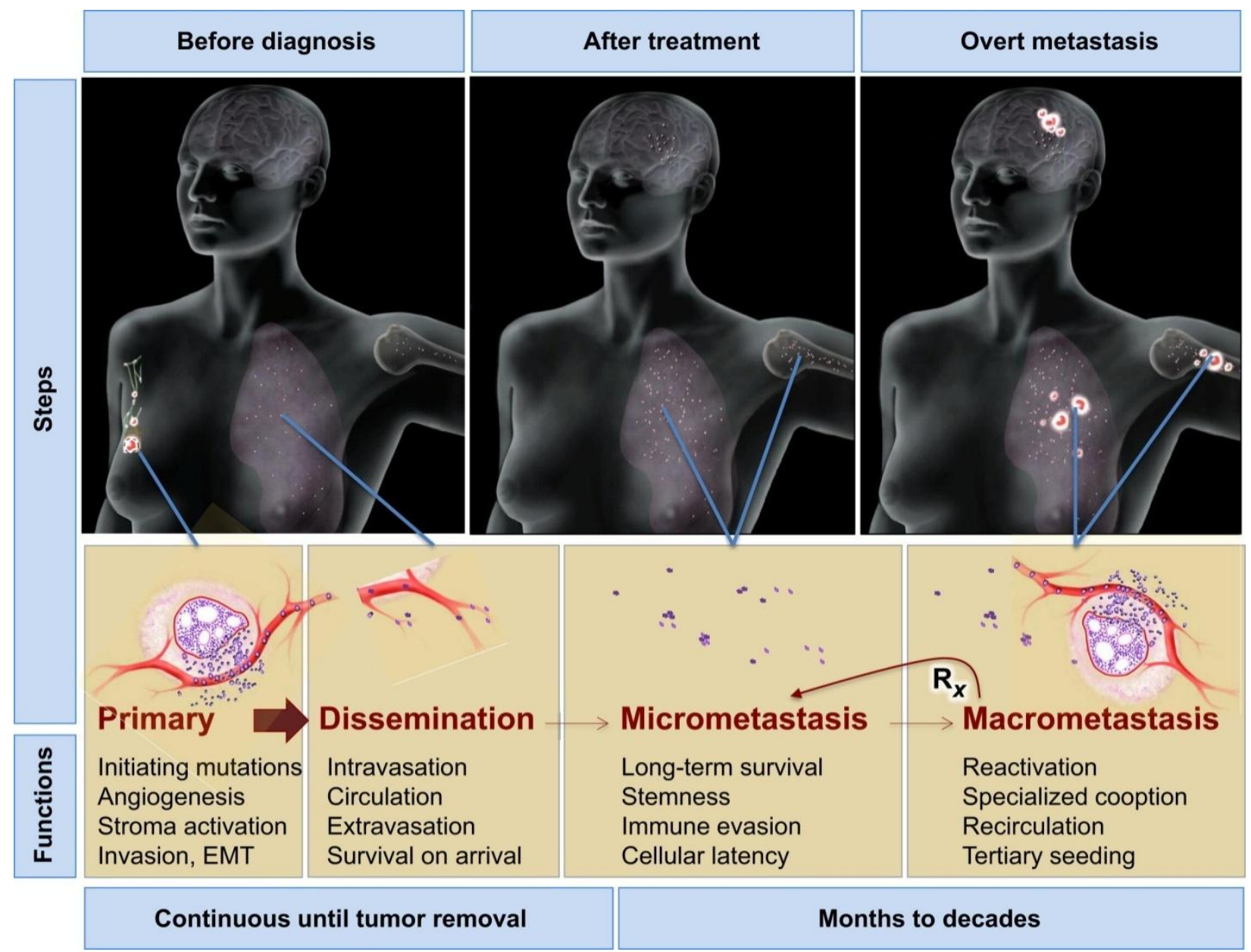

Figure 19. Steps and obstacles in metastasis. Used with permission from Vanharanta, S and Massagué, J Cancer Cell 2013(545). A sequence of steps involved in the metastasis of breast cancer, involving the events of primary tumor cells, dissemination, establishment of micrometastasis, and progression to macrometastasis. The dissemination of cells to distant sites can occur early in disease, whereas in most patients the development of macrometastasis occurs after initial treatment. Very few disseminated cells result in micrometastases, and most micrometastases do not progress to macrometastases. Critical bottlenecks in metastasis are considered to be later steps in the progression, such as the long-term survival and reactivation of cells at metastatic sites. 


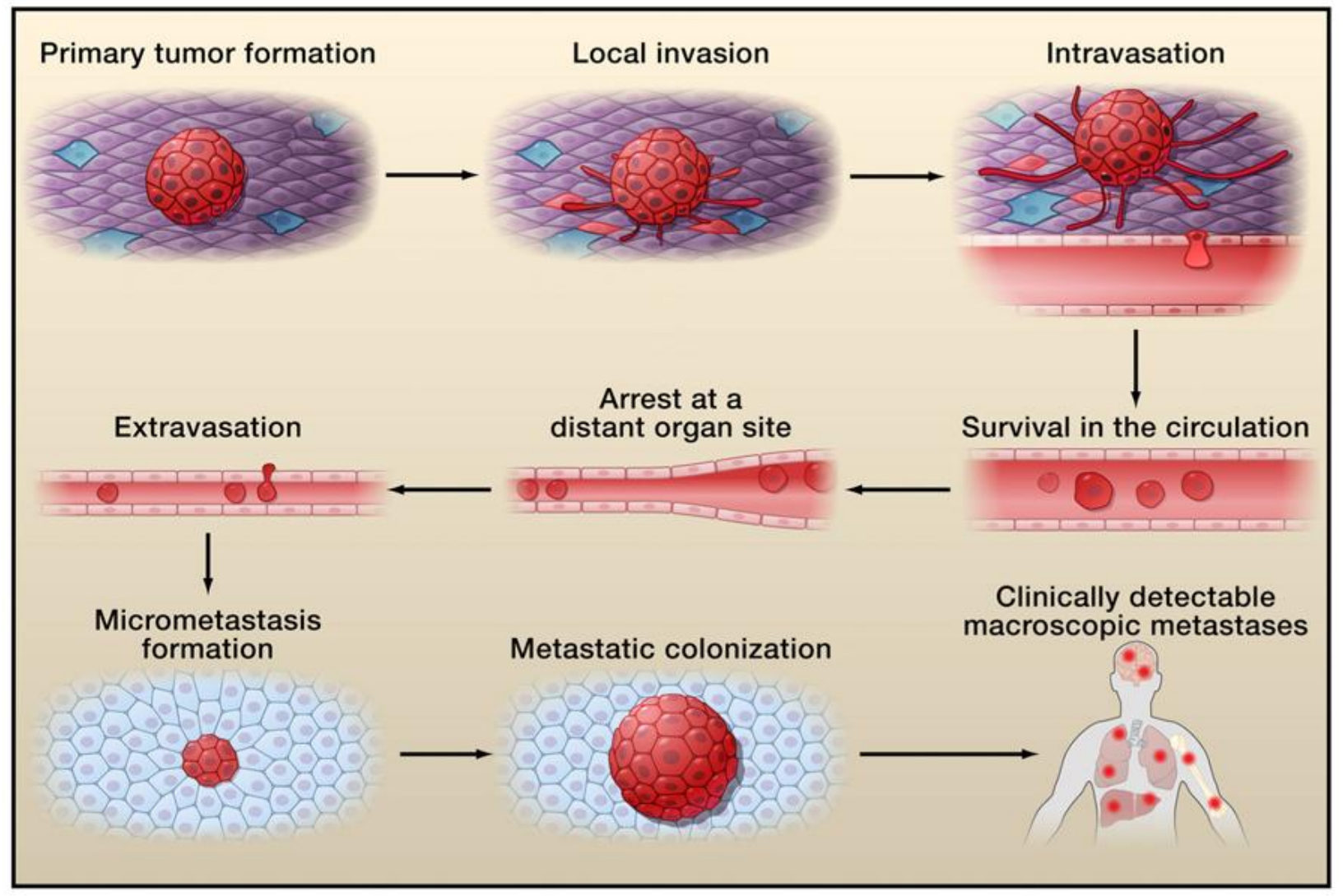

Figure 20. Circulating tumor cells in the metastatic cascade. Used with permission from Valastyan, S and Weinberg, RA Cell 2011 (543). An alternate view of metastasis highlights the role of tumor cells in circulation as an intermediate step in the metastatic process. It also possible for cancer cells to disseminate through the lymphatic system or via body cavities such as the peritoneum. Lymphatic vessels eventually drain into the bloodstream. The most common sites of distant metastasis in breast cancer are lungs, bone, liver, and brain. Of the tumor cells released into the bloodstream, the overwhelming majority are unable to form macrometastasis. 

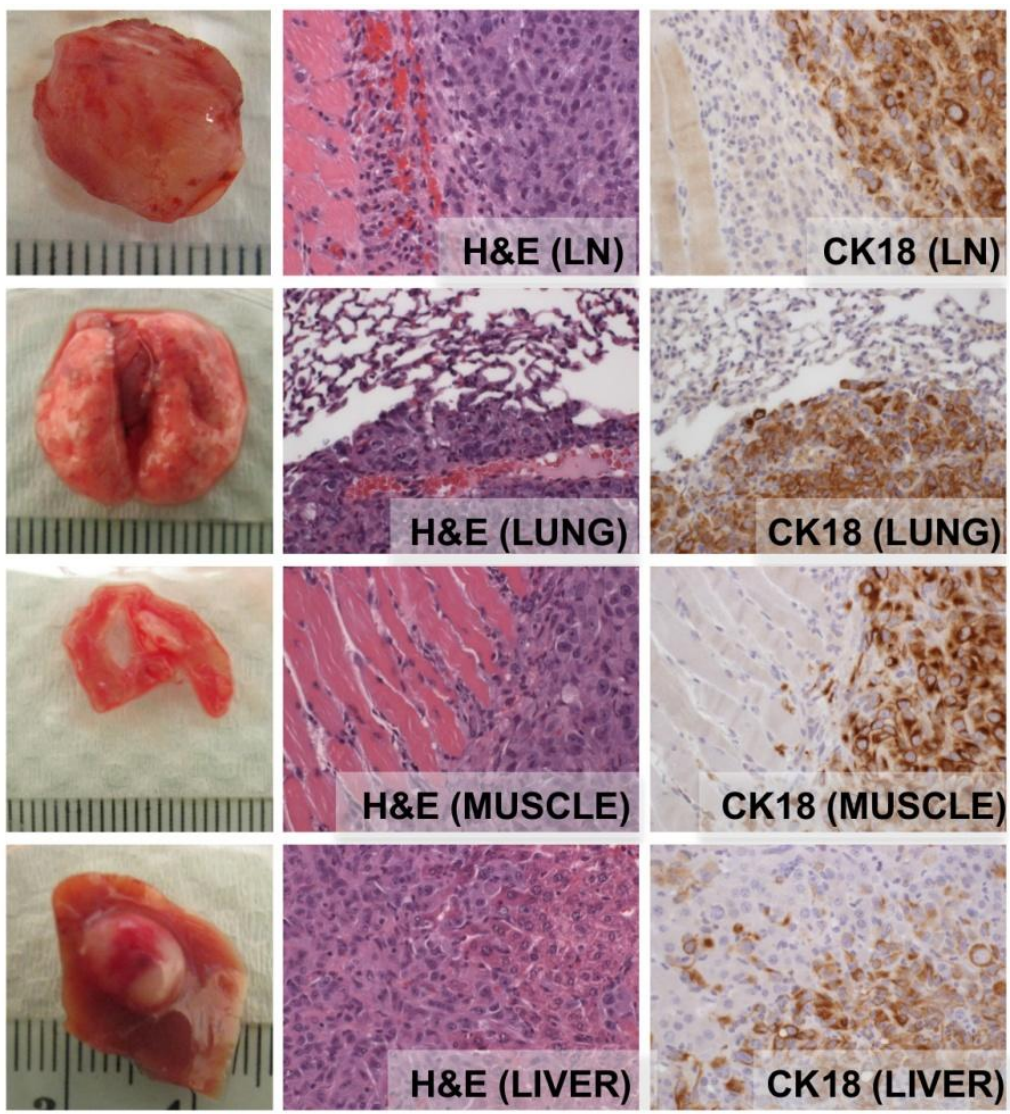

Figure 21. Spontaneous metastasis of MDA-MB-231 cells. Adapted from Iorns, E et al. PLoS One 2012 (537). Used under license (CC-BY). The MDA-MB-231 breast cancer cell line will spontaneously metastasize from primary orthotopic tumors in NSG mice. Panels illustrate spontaneous metastasis to axillary lymph nodes, lungs, skeletal muscle, and liver. Shown are gross specimens (left column), H\&E sections (center column), and cytokeratin 18 (CK18) by immunohistochemistry (right column). In contrast to surrounding tissue, tumor cells strongly express CK18. 

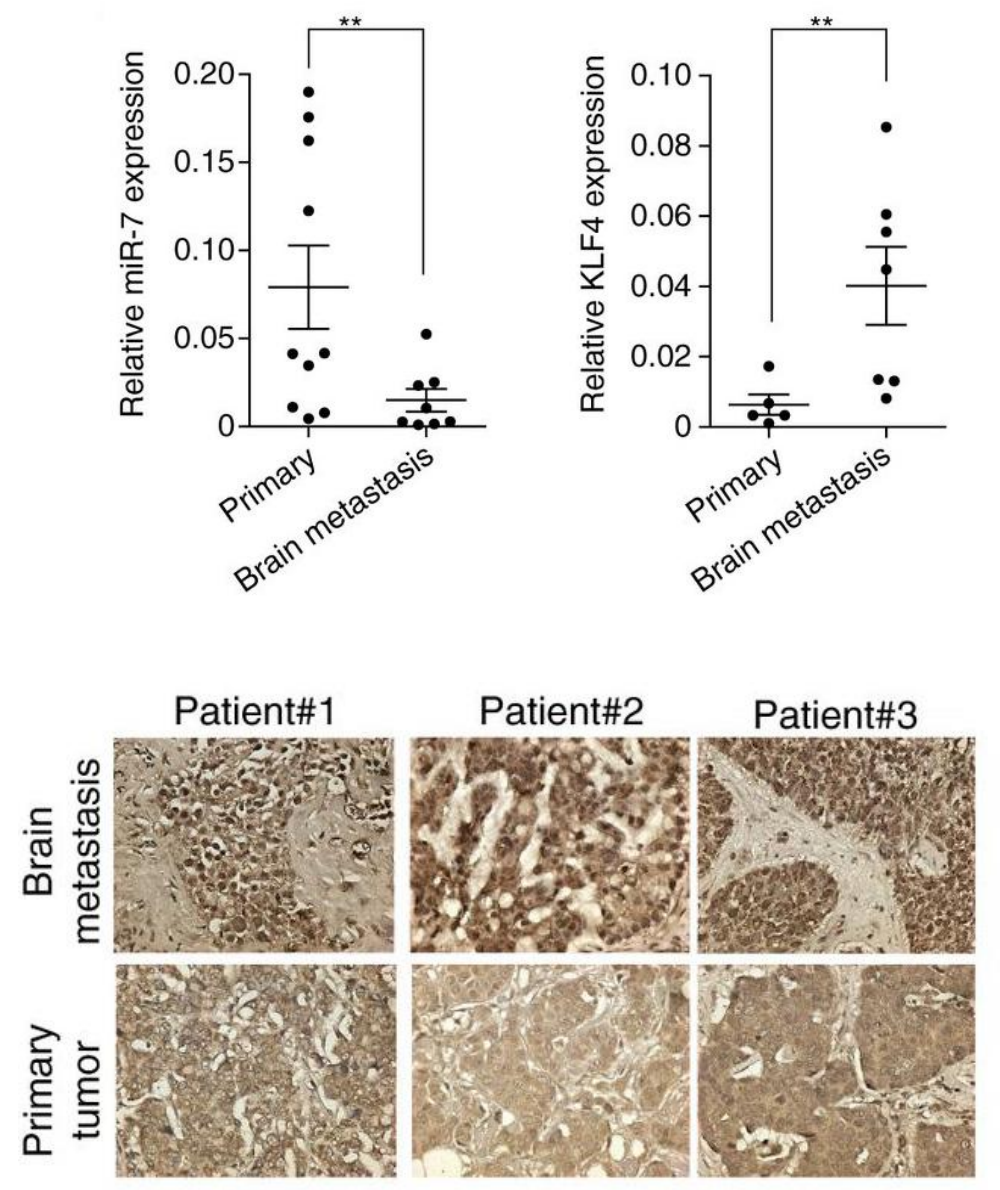

Figure 22. Expression of miR-7 and KLF4 in brain metastases of human breast cancer. From Okuda, H et al. Cancer Res 2013 (483). Adapted by permission from the American Association for Cancer Research: Okuda H et al, miR-7 suppresses brain metastasis of breast cancer stemlike cells by modulating KLF4, Cancer Research, 2013 February 15, vol 73. issue 4, 1434-44, doi: 10.1158/0008-5472.CAN-12-2037. Upper panels: Expression of miR-7 is decreased in brain metastases of breast cancer, whereas expression of KLF4 mRNA is increased. miR-7 is plotted relative to RNU48, and KLF4 is plotted relative to ACTB. Lower panels: Increased nuclear expression of KLF4 (immunohistochemistry) in brain metastases as compared to primary breast tumors. 


\section{References}

1. Nusslein-Volhard, C. and E. Wieschaus. 1980. Mutations affecting segment number and polarity in Drosophila. Nature 287:795-801.

2. Varjosalo, M. and J. Taipale. 2008. Hedgehog: functions and mechanisms. Genes Dev 22:2454-2472.

3. Huangfu, D. and K. V. Anderson. 2006. Signaling from Smo to Ci/Gli: conservation and divergence of Hedgehog pathways from Drosophila to vertebrates. Development 133:314.

4. Osterlund, T. and P. Kogerman. 2006. Hedgehog signalling: how to get from Smo to Ci and Gli. Trends Cell Biol 16:176-180.

5. Ekker, S. C., A. R. Ungar, P. Greenstein, D. P. von Kessler, J. A. Porter, R. T. Moon, and P. A. Beachy. 1995. Patterning activities of vertebrate hedgehog proteins in the developing eye and brain. Curr Biol 5:944-955.

6. Ingham, P. W. and A. P. McMahon. 2001. Hedgehog signaling in animal development: paradigms and principles. Genes Dev 15:3059-3087.

7. Hooper, J. E. and M. P. Scott. 1989. The Drosophila patched gene encodes a putative membrane protein required for segmental patterning. Cell 59:751-765.

8. Nakano, Y., I. Guerrero, A. Hidalgo, A. Taylor, J. R. Whittle, and P. W. Ingham. 1989. A protein with several possible membrane-spanning domains encoded by the Drosophila segment polarity gene patched. Nature 341:508-513.

9. Marigo, V., R. A. Davey, Y. Zuo, J. M. Cunningham, and C. J. Tabin. 1996. Biochemical evidence that patched is the Hedgehog receptor. Nature 384:176-179.

10. Forbes, A. J., Y. Nakano, A. M. Taylor, and P. W. Ingham. 1993. Genetic analysis of hedgehog signalling in the Drosophila embryo. Dev Suppl115-124.

11. Motoyama, J., T. Takabatake, K. Takeshima, and C. Hui. 1998. Ptch2, a second mouse Patched gene is co-expressed with Sonic hedgehog. Nat Genet 18:104-106. 
12. Zaphiropoulos, P. G., A. B. Unden, F. Rahnama, R. E. Hollingsworth, and R. Toftgard. 1999. PTCH2, a novel human patched gene, undergoing alternative splicing and upregulated in basal cell carcinomas. Cancer Res 59:787-792.

13. Robbins, D. J., D. L. Fei, and N. A. Riobo. 2012. The Hedgehog signal transduction network. Sci Signal 5:re6.

14. van den Heuvel, M. and P. W. Ingham. 1996. smoothened encodes a receptor-like serpentine protein required for hedgehog signalling. Nature 382:547-551.

15. Alcedo, J., M. Ayzenzon, O. T. Von, M. Noll, and J. E. Hooper. 1996. The Drosophila smoothened gene encodes a seven-pass membrane protein, a putative receptor for the hedgehog signal. Cell 86:221-232.

16. Corbit, K. C., P. Aanstad, V. Singla, A. R. Norman, D. Y. Stainier, and J. F. Reiter. 2005. Vertebrate Smoothened functions at the primary cilium. Nature 437:1018-1021.

17. Denef, N., D. Neubuser, L. Perez, and S. M. Cohen. 2000. Hedgehog induces opposite changes in turnover and subcellular localization of patched and smoothened. Cell 102:521-531.

18. Wang, Y., Z. Zhou, C. T. Walsh, and A. P. McMahon. 2009. Selective translocation of intracellular Smoothened to the primary cilium in response to Hedgehog pathway modulation. Proc Natl Acad Sci U S A 106:2623-2628.

19. McCabe, J. M. and D. J. Leahy. 2015. Smoothened goes molecular: new pieces in the hedgehog signaling puzzle. J Biol Chem 290:3500-3507.

20. Stottmann, R. W., A. Turbe-Doan, P. Tran, L. E. Kratz, J. L. Moran, R. I. Kelley, and D. R. Beier. 2011. Cholesterol metabolism is required for intracellular hedgehog signal transduction in vivo. PLoS Genet 7:e1002224.

21. Nachtergaele, S., L. K. Mydock, K. Krishnan, J. Rammohan, P. H. Schlesinger, D. F. Covey, and R. Rohatgi. 2012. Oxysterols are allosteric activators of the oncoprotein Smoothened. Nat Chem Biol 8:211-220.

22. Nedelcu, D., J. Liu, Y. Xu, C. Jao, and A. Salic. 2013. Oxysterol binding to the extracellular domain of Smoothened in Hedgehog signaling. Nat Chem Biol 9:557-564. 
23. Zhang, X. M., M. Ramalho-Santos, and A. P. McMahon. 2001. Smoothened mutants reveal redundant roles for Shh and Ihh signaling including regulation of L/R asymmetry by the mouse node. Cell 105:781-792.

24. Von Ohlen, T., D. Lessing, R. Nusse, and J. E. Hooper. 1997. Hedgehog signaling regulates transcription through cubitus interruptus, a sequence-specific DNA binding protein. Proc Natl Acad Sci U S A 94:2404-2409.

25. Orenic, T. V., D. C. Slusarski, K. L. Kroll, and R. A. Holmgren. 1990. Cloning and characterization of the segment polarity gene cubitus interruptus Dominant of Drosophila. Genes Dev 4:1053-1067.

26. Chen, M. H., C. W. Wilson, Y. J. Li, K. K. Law, C. S. Lu, R. Gacayan, X. Zhang, C. C. Hui, and P. T. Chuang. 2009. Cilium-independent regulation of Gli protein function by Sufu in Hedgehog signaling is evolutionarily conserved. Genes Dev 23:1910-1928.

27. Aikin, R. A., K. L. Ayers, and P. P. Therond. 2008. The role of kinases in the Hedgehog signalling pathway. EMBO Rep 9:330-336.

28. He, M., R. Subramanian, F. Bangs, T. Omelchenko, K. F. Liem, Jr., T. M. Kapoor, and K. V. Anderson. 2014. The kinesin-4 protein Kif7 regulates mammalian Hedgehog signalling by organizing the cilium tip compartment. Nat Cell Biol 16:663-672.

29. Tukachinsky, H., L. V. Lopez, and A. Salic. 2010. A mechanism for vertebrate Hedgehog signaling: recruitment to cilia and dissociation of $\mathrm{SuFu}-\mathrm{Gli}$ protein complexes. J Cell Biol 191:415-428.

30. Jiang, J. and G. Struhl. 1998. Regulation of the Hedgehog and Wingless signalling pathways by the F-box/WD40-repeat protein Slimb. Nature 391:493-496.

31. Fujiwara, T., M. Suzuki, A. Tanigami, T. Ikenoue, M. Omata, T. Chiba, and K. Tanaka. 1999. The BTRC gene, encoding a human F-box/WD40-repeat protein, maps to chromosome 10q24-q25. Genomics 58:104-105.

32. Ji, Z., F. C. Mei, J. Xie, and X. Cheng. 2007. Oncogenic KRAS activates hedgehog signaling pathway in pancreatic cancer cells. J Biol Chem 282:14048-14055.

33. Stecca, B., C. Mas, V. Clement, M. Zbinden, R. Correa, V. Piguet, F. Beermann, and I. A. Ruiz. 2007. Melanomas require HEDGEHOG-GLI signaling regulated by interactions 
between GLI1 and the RAS-MEK/AKT pathways. Proc Natl Acad Sci U S A 104:58955900 .

34. Nolan-Stevaux, O., J. Lau, M. L. Truitt, G. C. Chu, M. Hebrok, M. E. Fernandez-Zapico, and D. Hanahan. 2009. GLI1 is regulated through Smoothened-independent mechanisms in neoplastic pancreatic ducts and mediates PDAC cell survival and transformation. Genes Dev 23:24-36.

35. Huntzicker, E. G., I. S. Estay, H. Zhen, L. A. Lokteva, P. K. Jackson, and A. E. Oro. 2006. Dual degradation signals control Gli protein stability and tumor formation. Genes Dev 20:276-281.

36. Shimeld, S. M., H. M. van den, R. Dawber, and J. Briscoe. 2007. An amphioxus Gli gene reveals conservation of midline patterning and the evolution of hedgehog signalling diversity in chordates. PLoS One 2:e864.

37. Hui, C. C. and S. Angers. 2011. Gli proteins in development and disease. Annu Rev Cell Dev Biol 27:513-537.

38. Park, H. L., C. Bai, K. A. Platt, M. P. Matise, A. Beeghly, C. C. Hui, M. Nakashima, and A. L. Joyner. 2000. Mouse Gli1 mutants are viable but have defects in SHH signaling in combination with a Gli2 mutation. Development 127:1593-1605.

39. Dai, P., H. Akimaru, Y. Tanaka, T. Maekawa, M. Nakafuku, and S. Ishii. 1999. Sonic Hedgehog-induced activation of the Gli1 promoter is mediated by GLI3. J Biol Chem 274:8143-8152.

40. Altaba, A., C. Mas, and B. Stecca. 2007. The Gli code: an information nexus regulating cell fate, stemness and cancer. Trends Cell Biol 17:438-447.

41. Hu, M. C., R. Mo, S. Bhella, C. W. Wilson, P. T. Chuang, C. C. Hui, and N. D. Rosenblum. 2006. GLI3-dependent transcriptional repression of Gli1, Gli2 and kidney patterning genes disrupts renal morphogenesis. Development 133:569-578.

42. Regl, G., G. W. Neill, T. Eichberger, M. Kasper, M. S. Ikram, J. Koller, H. Hintner, A. G. Quinn, A. M. Frischauf, and F. Aberger. 2002. Human GLI2 and GLI1 are part of a positive feedback mechanism in Basal Cell Carcinoma. Oncogene 21:5529-5539. 
43. Kinzler, K. W., S. H. Bigner, D. D. Bigner, J. M. Trent, M. L. Law, S. J. O'Brien, A. J. Wong, and B. Vogelstein. 1987. Identification of an amplified, highly expressed gene in a human glioma. Science 236:70-73.

44. Kinzler, K. W., J. M. Ruppert, S. H. Bigner, and B. Vogelstein. 1988. The GLI gene is a member of the Kruppel family of zinc finger proteins. Nature 332:371-374.

45. Ruppert, J. M., K. W. Kinzler, A. J. Wong, S. H. Bigner, F. T. Kao, M. L. Law, H. N. Seuanez, S. J. O'Brien, and B. Vogelstein. 1988. The GLI-Kruppel family of human genes. Mol Cell Biol 8:3104-3113.

46. Ruppert, J. M., B. Vogelstein, K. Arheden, and K. W. Kinzler. 1990. GLI3 encodes a 190-kilodalton protein with multiple regions of GLI similarity. Mol Cell Biol 10:54085415.

47. Krishna, S. S., I. Majumdar, and N. V. Grishin. 2003. Structural classification of zinc fingers: survey and summary. Nucleic Acids Res 31:532-550.

48. Sasaki, H., C. Hui, M. Nakafuku, and H. Kondoh. 1997. A binding site for Gli proteins is essential for HNF-3beta floor plate enhancer activity in transgenics and can respond to Shh in vitro. Development 124:1313-1322.

49. Yoon, J. W., C. Z. Liu, J. T. Yang, R. Swart, P. Iannaccone, and D. Walterhouse. 1998. GLI activates transcription through a herpes simplex viral protein 16-like activation domain. J Biol Chem 273:3496-3501.

50. Schrader, E. K., K. G. Harstad, R. A. Holmgren, and A. Matouschek. 2011. A three-part signal governs differential processing of Gli1 and Gli3 proteins by the proteasome. J Biol Chem 286:39051-39058.

51. Wang, X. Q. and J. A. Rothnagel. 2001. Post-transcriptional regulation of the gli1 oncogene by the expression of alternative 5' untranslated regions. J Biol Chem 276:13111316.

52. Zaphiropoulos, P. G. 2012. Genetic variations and alternative splicing: the Glioma associated oncogene 1, GLI1. Front Genet 3:119.

53. Shimokawa, T., U. Tostar, M. Lauth, R. Palaniswamy, M. Kasper, R. Toftgard, and P. G. Zaphiropoulos. 2008. Novel human glioma-associated oncogene 1 (GLI1) splice variants 
reveal distinct mechanisms in the terminal transduction of the hedgehog signal. J Biol Chem 283:14345-14354.

54. Mao, J., P. Maye, P. Kogerman, F. J. Tejedor, R. Toftgard, W. Xie, G. Wu, and D. Wu. 2002. Regulation of Gli1 transcriptional activity in the nucleus by Dyrk1. J Biol Chem 277:35156-35161.

55. Lo, H. W., H. Zhu, X. Cao, A. Aldrich, and F. li-Osman. 2009. A novel splice variant of GLI1 that promotes glioblastoma cell migration and invasion. Cancer Res 69:6790-6798.

56. Cao, X., J. Geradts, M. W. Dewhirst, and H. W. Lo. 2012. Upregulation of VEGF-A and CD24 gene expression by the tGLI1 transcription factor contributes to the aggressive behavior of breast cancer cells. Oncogene 31:104-115.

57. Carpenter, R. L. and H. W. Lo. 2012. Hedgehog pathway and GLI1 isoforms in human cancer. Discov Med 13:105-113.

58. Bieniossek, C., G. Papai, C. Schaffitzel, F. Garzoni, M. Chaillet, E. Scheer, P. Papadopoulos, L. Tora, P. Schultz, and I. Berger. 2013. The architecture of human general transcription factor TFIID core complex. Nature 493:699-702.

59. Bosco-Clement, G., F. Zhang, Z. Chen, H. M. Zhou, H. Li, I. Mikami, T. Hirata, A. Yagui-Beltran, N. Lui, H. T. Do, T. Cheng, H. H. Tseng, H. Choi, L. T. Fang, I. J. Kim, D. Yue, C. Wang, Q. Zheng, N. Fujii, M. Mann, D. M. Jablons, and B. He. 2014. Targeting Gli transcription activation by small molecule suppresses tumor growth. Oncogene 33:2087-2097.

60. Canettieri, G., M. L. Di, A. Greco, S. Coni, L. Antonucci, P. Infante, L. Pietrosanti, S. E. De, E. Ferretti, E. Miele, M. Pelloni, S. G. De, E. M. Pedone, P. Gallinari, A. Giorgi, C. Steinkuhler, L. Vitagliano, C. Pedone, M. E. Schinin, I. Screpanti, and A. Gulino. 2010. Histone deacetylase and Cullin3-REN(KCTD11) ubiquitin ligase interplay regulates Hedgehog signalling through Gli acetylation. Nat Cell Biol 12:132-142.

61. Argenti, B., R. Gallo, M. L. Di, E. Ferretti, M. Napolitano, S. Canterini, S. E. De, A. Greco, M. T. Fiorenza, M. Maroder, I. Screpanti, E. Alesse, and A. Gulino. 2005. Hedgehog antagonist REN(KCTD11) regulates proliferation and apoptosis of developing granule cell progenitors. J Neurosci 25:8338-8346.

62. Di Marcotullio, L., E. Ferretti, S. E. De, B. Argenti, C. Mincione, F. Zazzeroni, R. Gallo, L. Masuelli, M. Napolitano, M. Maroder, A. Modesti, F. Giangaspero, I. Screpanti, E. 
Alesse, and A. Gulino. 2004. REN(KCTD11) is a suppressor of Hedgehog signaling and is deleted in human medulloblastoma. Proc Natl Acad Sci U S A 101:10833-10838.

63. Dai, P., T. Shinagawa, T. Nomura, J. Harada, S. C. Kaul, R. Wadhwa, M. M. Khan, H. Akimaru, H. Sasaki, C. Colmenares, and S. Ishii. 2002. Ski is involved in transcriptional regulation by the repressor and full-length forms of Gli3. Genes Dev 16:2843-2848.

64. Cheng, S. Y. and J. M. Bishop. 2002. Suppressor of Fused represses Gli-mediated transcription by recruiting the SAP18-mSin3 corepressor complex. Proc Natl Acad Sci U S A 99:5442-5447.

65. Jagani, Z., E. L. Mora-Blanco, C. G. Sansam, E. S. McKenna, B. Wilson, D. Chen, J. Klekota, P. Tamayo, P. T. Nguyen, M. Tolstorukov, P. J. Park, Y. J. Cho, K. Hsiao, S. Buonamici, S. L. Pomeroy, J. P. Mesirov, H. Ruffner, T. Bouwmeester, S. J. Luchansky, J. Murtie, J. F. Kelleher, M. Warmuth, W. R. Sellers, C. W. Roberts, and M. Dorsch. 2010. Loss of the tumor suppressor Snf5 leads to aberrant activation of the Hedgehog-Gli pathway. Nat Med 16:1429-1433.

66. Cox, B., J. Briscoe, and F. Ulloa. 2010. SUMOylation by Pias1 regulates the activity of the Hedgehog dependent Gli transcription factors. PLoS One 5:e11996.

67. Aza-Blanc, P., F. A. Ramirez-Weber, M. P. Laget, C. Schwartz, and T. B. Kornberg. 1997. Proteolysis that is inhibited by hedgehog targets Cubitus interruptus protein to the nucleus and converts it to a repressor. Cell 89:1043-1053.

68. Smelkinson, M. G. and D. Kalderon. 2006. Processing of the Drosophila hedgehog signaling effector $\mathrm{Ci}-155$ to the repressor $\mathrm{Ci}-75$ is mediated by direct binding to the $\mathrm{SCF}$ component Slimb. Curr Biol 16:110-116.

69. Tian, L., R. A. Holmgren, and A. Matouschek. 2005. A conserved processing mechanism regulates the activity of transcription factors Cubitus interruptus and NF-kappaB. Nat Struct Mol Biol 12:1045-1053.

70. Methot, N. and K. Basler. 1999. Hedgehog controls limb development by regulating the activities of distinct transcriptional activator and repressor forms of Cubitus interruptus. Cell 96:819-831.

71. Smelkinson, M. G., Q. Zhou, and D. Kalderon. 2007. Regulation of Ci-SCFSlimb binding, $\mathrm{Ci}$ proteolysis, and hedgehog pathway activity by $\mathrm{Ci}$ phosphorylation. Dev Cell 13:481-495. 
72. Tempe, D., M. Casas, S. Karaz, M. F. Blanchet-Tournier, and J. P. Concordet. 2006. Multisite protein kinase A and glycogen synthase kinase 3 beta phosphorylation leads to Gli3 ubiquitination by SCFbetaTrCP. Mol Cell Biol 26:4316-4326.

73. Wang, B., J. F. Fallon, and P. A. Beachy. 2000. Hedgehog-regulated processing of Gli3 produces an anterior/posterior repressor gradient in the developing vertebrate limb. Cell 100:423-434.

74. Wang, B. and Y. Li. 2006. Evidence for the direct involvement of \{ beta $\} \operatorname{TrCP}$ in Gli3 protein processing. Proc Natl Acad Sci U S A 103:33-38.

75. Pan, Y., C. Wang, and B. Wang. 2009. Phosphorylation of Gli2 by protein kinase A is required for Gli2 processing and degradation and the Sonic Hedgehog-regulated mouse development. Dev Biol 326:177-189.

76. Pan, Y., C. B. Bai, A. L. Joyner, and B. Wang. 2006. Sonic hedgehog signaling regulates Gli2 transcriptional activity by suppressing its processing and degradation. Mol Cell Biol 26:3365-3377.

77. Pan, Y. and B. Wang. 2007. A novel protein-processing domain in Gli2 and Gli3 differentially blocks complete protein degradation by the proteasome. J Biol Chem 282:10846-10852.

78. Kent, D., E. W. Bush, and J. E. Hooper. 2006. Roadkill attenuates Hedgehog responses through degradation of Cubitus interruptus. Development 133:2001-2010.

79. Zhang, Q., L. Zhang, B. Wang, C. Y. Ou, C. T. Chien, and J. Jiang. 2006. A hedgehoginduced BTB protein modulates hedgehog signaling by degrading Ci/Gli transcription factor. Dev Cell 10:719-729.

80. Seong, K. H., H. Akimaru, P. Dai, T. Nomura, M. Okada, and S. Ishii. 2010. Inhibition of the nuclear import of cubitus interruptus by roadkill in the presence of strong hedgehog signal. PLoS One 5:e15365.

81. Seong, K. H. and S. Ishii. 2013. Su(fu) switches Rdx functions to fine-tune hedgehog signaling in the Drosophila wing disk. Genes Cells 18:66-78. 
82. Zhang, Q., Q. Shi, Y. Chen, T. Yue, S. Li, B. Wang, and J. Jiang. 2009. Multiple Ser/Thrrich degrons mediate the degradation of $\mathrm{Ci} / \mathrm{Gli}$ by the Cul3-HIB/SPOP E3 ubiquitin ligase. Proc Natl Acad Sci U S A 106:21191-21196.

83. Di Marcotullio, L., E. Ferretti, A. Greco, S. E. De, A. Po, M. A. Sico, M. Alimandi, G. Giannini, M. Maroder, I. Screpanti, and A. Gulino. 2006. Numb is a suppressor of Hedgehog signalling and targets Gli1 for Itch-dependent ubiquitination. Nat Cell Biol 8:1415-1423.

84. Di Marcotullio, L., A. Greco, D. Mazza, G. Canettieri, L. Pietrosanti, P. Infante, S. Coni, M. Moretti, S. E. De, E. Ferretti, I. Screpanti, and A. Gulino. 2011. Numb activates the E3 ligase Itch to control Gli1 function through a novel degradation signal. Oncogene 30:65-76.

85. Agren, M., P. Kogerman, M. I. Kleman, M. Wessling, and R. Toftgard. 2004. Expression of the PTCH1 tumor suppressor gene is regulated by alternative promoters and a single functional Gli-binding site. Gene 330:101-114.

86. Lee, E. Y., H. Ji, Z. Ouyang, B. Zhou, W. Ma, S. A. Vokes, A. P. McMahon, W. H. Wong, and M. P. Scott. 2010. Hedgehog pathway-regulated gene networks in cerebellum development and tumorigenesis. Proc Natl Acad Sci U S A 107:9736-9741.

87. Vokes, S. A., H. Ji, S. McCuine, T. Tenzen, S. Giles, S. Zhong, W. J. Longabaugh, E. H. Davidson, W. H. Wong, and A. P. McMahon. 2007. Genomic characterization of Gliactivator targets in sonic hedgehog-mediated neural patterning. Development 134:19771989.

88. Vokes, S. A., H. Ji, W. H. Wong, and A. P. McMahon. 2008. A genome-scale analysis of the cis-regulatory circuitry underlying sonic hedgehog-mediated patterning of the mammalian limb. Genes Dev 22:2651-2663.

89. Zwerner, J. P., J. Joo, K. L. Warner, L. Christensen, S. Hu-Lieskovan, T. J. Triche, and W. A. May. 2008. The EWS/FLI1 oncogenic transcription factor deregulates GLI1. Oncogene 27:3282-3291.

90. Beauchamp, E., G. Bulut, O. Abaan, K. Chen, A. Merchant, W. Matsui, Y. Endo, J. S. Rubin, J. Toretsky, and A. Uren. 2009. GLI1 is a direct transcriptional target of EWSFLI1 oncoprotein. J Biol Chem 284:9074-9082. 
91. Joo, J., L. Christensen, K. Warner, L. States, H. G. Kang, K. Vo, E. R. Lawlor, and W. A. May. 2009. GLI1 is a central mediator of EWS/FLI1 signaling in Ewing tumors. PLoS One 4:e7608.

92. Roberts, W. M., E. C. Douglass, S. C. Peiper, P. J. Houghton, and A. T. Look. 1989. Amplification of the gli gene in childhood sarcomas. Cancer Res 49:5407-5413.

93. Lomas, A., J. Leonardi-Bee, and F. Bath-Hextall. 2012. A systematic review of worldwide incidence of nonmelanoma skin cancer. Br. J Dermatol 166:1069-1080.

94. Yang, L., G. Xie, Q. Fan, and J. Xie. 2010. Activation of the hedgehog-signaling pathway in human cancer and the clinical implications. Oncogene 29:469-481.

95. Gorlin, R. J. 1995. Nevoid basal cell carcinoma syndrome. Dermatol Clin 13:113-125.

96. Hahn, H., C. Wicking, P. G. Zaphiropoulous, M. R. Gailani, S. Shanley, A. Chidambaram, I. Vorechovsky, E. Holmberg, A. B. Unden, S. Gillies, K. Negus, I. Smyth, C. Pressman, D. J. Leffell, B. Gerrard, A. M. Goldstein, M. Dean, R. Toftgard, G. Chenevix-Trench, B. Wainwright, and A. E. Bale. 1996. Mutations of the human homolog of Drosophila patched in the nevoid basal cell carcinoma syndrome. Cell 85:841-851.

97. Gailani, M. R., M. Stahle-Backdahl, D. J. Leffell, M. Glynn, P. G. Zaphiropoulos, C. Pressman, A. B. Unden, M. Dean, D. E. Brash, A. E. Bale, and R. Toftgard. 1996. The role of the human homologue of Drosophila patched in sporadic basal cell carcinomas. Nat Genet 14:78-81.

98. Xie, J., M. Murone, S. M. Luoh, A. Ryan, Q. Gu, C. Zhang, J. M. Bonifas, C. W. Lam, M. Hynes, A. Goddard, A. Rosenthal, E. H. Epstein, Jr., and F. J. de Sauvage. 1998. Activating Smoothened mutations in sporadic basal-cell carcinoma. Nature 391:90-92.

99. Pietsch, T., A. Waha, A. Koch, J. Kraus, S. Albrecht, J. Tonn, N. Sorensen, F. Berthold, B. Henk, N. Schmandt, H. K. Wolf, D. A. von, B. Wainwright, G. Chenevix-Trench, O. D. Wiestler, and C. Wicking. 1997. Medulloblastomas of the desmoplastic variant carry mutations of the human homologue of Drosophila patched. Cancer Res 57:2085-2088.

100. Raffel, C., R. B. Jenkins, L. Frederick, D. Hebrink, B. Alderete, D. W. Fults, and C. D. James. 1997. Sporadic medulloblastomas contain PTCH mutations. Cancer Res 57:842845. 
101. Wolter, M., J. Reifenberger, C. Sommer, T. Ruzicka, and G. Reifenberger. 1997. Mutations in the human homologue of the Drosophila segment polarity gene patched (PTCH) in sporadic basal cell carcinomas of the skin and primitive neuroectodermal tumors of the central nervous system. Cancer Res 57:2581-2585.

102. Xie, J., R. L. Johnson, X. Zhang, J. W. Bare, F. M. Waldman, P. H. Cogen, A. G. Menon, R. S. Warren, L. C. Chen, M. P. Scott, and E. H. Epstein, Jr. 1997. Mutations of the PATCHED gene in several types of sporadic extracutaneous tumors. Cancer Res 57:2369-2372.

103. Zurawel, R. H., C. Allen, S. Chiappa, W. Cato, J. Biegel, P. Cogen, S. F. de, and C. Raffel. 2000. Analysis of PTCH/SMO/SHH pathway genes in medulloblastoma. Genes Chromosomes Cancer 27:44-51.

104. Berman, D. M., S. S. Karhadkar, A. Maitra, O. R. Montes De, M. R. Gerstenblith, K. Briggs, A. R. Parker, Y. Shimada, J. R. Eshleman, D. N. Watkins, and P. A. Beachy. 2003. Widespread requirement for Hedgehog ligand stimulation in growth of digestive tract tumours. Nature 425:846-851.

105. Hezel, A. F., A. C. Kimmelman, B. Z. Stanger, N. Bardeesy, and R. A. Depinho. 2006. Genetics and biology of pancreatic ductal adenocarcinoma. Genes Dev 20:1218-1249.

106. Almoguera, C., D. Shibata, K. Forrester, J. Martin, N. Arnheim, and M. Perucho. 1988. Most human carcinomas of the exocrine pancreas contain mutant c-K-ras genes. Cell 53:549-554.

107. Smit, V. T., A. J. Boot, A. M. Smits, G. J. Fleuren, C. J. Cornelisse, and J. L. Bos. 1988. KRAS codon 12 mutations occur very frequently in pancreatic adenocarcinomas. Nucleic Acids Res 16:7773-7782.

108. Ruggeri, B., S. Y. Zhang, J. Caamano, M. DiRado, S. D. Flynn, and A. J. Klein-Szanto. 1992. Human pancreatic carcinomas and cell lines reveal frequent and multiple alterations in the p53 and Rb-1 tumor-suppressor genes. Oncogene 7:1503-1511.

109. Scarpa, A., P. Capelli, K. Mukai, G. Zamboni, T. Oda, C. Iacono, and S. Hirohashi. 1993. Pancreatic adenocarcinomas frequently show p53 gene mutations. Am J Pathol 142:15341543.

110. Caldas, C., S. A. Hahn, L. T. da Costa, M. S. Redston, M. Schutte, A. B. Seymour, C. L. Weinstein, R. H. Hruban, C. J. Yeo, and S. E. Kern. 1994. Frequent somatic mutations 
and homozygous deletions of the p16 (MTS1) gene in pancreatic adenocarcinoma. Nat Genet 8:27-32.

111. Hahn, S. A., M. Schutte, A. T. Hoque, C. A. Moskaluk, L. T. da Costa, E. Rozenblum, C. L. Weinstein, A. Fischer, C. J. Yeo, R. H. Hruban, and S. E. Kern. 1996. DPC4, a candidate tumor suppressor gene at human chromosome 18q21.1. Science 271:350-353.

112. Jones, S., X. Zhang, D. W. Parsons, J. C. Lin, R. J. Leary, P. Angenendt, P. Mankoo, H. Carter, H. Kamiyama, A. Jimeno, S. M. Hong, B. Fu, M. T. Lin, E. S. Calhoun, M. Kamiyama, K. Walter, T. Nikolskaya, Y. Nikolsky, J. Hartigan, D. R. Smith, M. Hidalgo, S. D. Leach, A. P. Klein, E. M. Jaffee, M. Goggins, A. Maitra, C. Iacobuzio-Donahue, J. R. Eshleman, S. E. Kern, R. H. Hruban, R. Karchin, N. Papadopoulos, G. Parmigiani, B. Vogelstein, V. E. Velculescu, and K. W. Kinzler. 2008. Core signaling pathways in human pancreatic cancers revealed by global genomic analyses. Science 321:1801-1806.

113. Eser, S., A. Schnieke, G. Schneider, and D. Saur. 2014. Oncogenic KRAS signalling in pancreatic cancer. Br. J Cancer 111:817-822.

114. Tuveson, D. A., A. T. Shaw, N. A. Willis, D. P. Silver, E. L. Jackson, S. Chang, K. L. Mercer, R. Grochow, H. Hock, D. Crowley, S. R. Hingorani, T. Zaks, C. King, M. A. Jacobetz, L. Wang, R. T. Bronson, S. H. Orkin, R. A. Depinho, and T. Jacks. 2004. Endogenous oncogenic K-ras(G12D) stimulates proliferation and widespread neoplastic and developmental defects. Cancer Cell 5:375-387.

115. Hingorani, S. R., E. F. Petricoin, A. Maitra, V. Rajapakse, C. King, M. A. Jacobetz, S. Ross, T. P. Conrads, T. D. Veenstra, B. A. Hitt, Y. Kawaguchi, D. Johann, L. A. Liotta, H. C. Crawford, M. E. Putt, T. Jacks, C. V. Wright, R. H. Hruban, A. M. Lowy, and D. A. Tuveson. 2003. Preinvasive and invasive ductal pancreatic cancer and its early detection in the mouse. Cancer Cell 4:437-450.

116. Bardeesy, N., A. J. Aguirre, G. C. Chu, K. H. Cheng, L. V. Lopez, A. F. Hezel, B. Feng, C. Brennan, R. Weissleder, U. Mahmood, D. Hanahan, M. S. Redston, L. Chin, and R. A. Depinho. 2006. Both p16(Ink4a) and the p19(Arf)-p53 pathway constrain progression of pancreatic adenocarcinoma in the mouse. Proc Natl Acad Sci U S A 103:5947-5952.

117. Hingorani, S. R., L. Wang, A. S. Multani, C. Combs, T. B. Deramaudt, R. H. Hruban, A. K. Rustgi, S. Chang, and D. A. Tuveson. 2005. Trp53R172H and KrasG12D cooperate to promote chromosomal instability and widely metastatic pancreatic ductal adenocarcinoma in mice. Cancer Cell 7:469-483. 
118. Collins, M. A., F. Bednar, Y. Zhang, J. C. Brisset, S. Galban, C. J. Galban, S. Rakshit, K. S. Flannagan, N. V. Adsay, and M. M. Pasca di. 2012. Oncogenic Kras is required for both the initiation and maintenance of pancreatic cancer in mice. J Clin Invest 122:639653.

119. Thayer, S. P., M. P. di Magliano, P. W. Heiser, C. M. Nielsen, D. J. Roberts, G. Y. Lauwers, Y. P. Qi, S. Gysin, C. C. Fernandez-del, V. Yajnik, B. Antoniu, M. McMahon, A. L. Warshaw, and M. Hebrok. 2003. Hedgehog is an early and late mediator of pancreatic cancer tumorigenesis. Nature 425:851-856.

120. Pasca di Magliano, M., S. Sekine, A. Ermilov, J. Ferris, A. A. Dlugosz, and M. Hebrok. 2006. Hedgehog/Ras interactions regulate early stages of pancreatic cancer. Genes Dev 20:3161-3173.

121. Feldmann, G., S. Dhara, V. Fendrich, D. Bedja, R. Beaty, M. Mullendore, C. Karikari, H. Alvarez, C. Iacobuzio-Donahue, A. Jimeno, K. L. Gabrielson, W. Matsui, and A. Maitra. 2007. Blockade of hedgehog signaling inhibits pancreatic cancer invasion and metastases: a new paradigm for combination therapy in solid cancers. Cancer Res 67:2187-2196.

122. Feldmann, G., V. Fendrich, K. McGovern, D. Bedja, S. Bisht, H. Alvarez, J. B. Koorstra, N. Habbe, C. Karikari, M. Mullendore, K. L. Gabrielson, R. Sharma, W. Matsui, and A. Maitra. 2008. An orally bioavailable small-molecule inhibitor of Hedgehog signaling inhibits tumor initiation and metastasis in pancreatic cancer. Mol Cancer Ther 7:27252735.

123. Feldmann, G., N. Habbe, S. Dhara, S. Bisht, H. Alvarez, V. Fendrich, R. Beaty, M. Mullendore, C. Karikari, N. Bardeesy, M. M. Ouellette, W. Yu, and A. Maitra. 2008. Hedgehog inhibition prolongs survival in a genetically engineered mouse model of pancreatic cancer. Gut 57:1420-1430.

124. Morton, J. P., M. E. Mongeau, D. S. Klimstra, J. P. Morris, Y. C. Lee, Y. Kawaguchi, C. V. Wright, M. Hebrok, and B. C. Lewis. 2007. Sonic hedgehog acts at multiple stages during pancreatic tumorigenesis. Proc Natl Acad Sci U S A 104:5103-5108.

125. Yauch, R. L., S. E. Gould, S. J. Scales, T. Tang, H. Tian, C. P. Ahn, D. Marshall, L. Fu, T. Januario, D. Kallop, M. Nannini-Pepe, K. Kotkow, J. C. Marsters, L. L. Rubin, and F. J. de Sauvage. 2008. A paracrine requirement for hedgehog signalling in cancer. Nature 455:406-410. 
126. Bailey, J. M., A. M. Mohr, and M. A. Hollingsworth. 2009. Sonic hedgehog paracrine signaling regulates metastasis and lymphangiogenesis in pancreatic cancer. Oncogene 28:3513-3525.

127. Olive, K. P., M. A. Jacobetz, C. J. Davidson, A. Gopinathan, D. McIntyre, D. Honess, B. Madhu, M. A. Goldgraben, M. E. Caldwell, D. Allard, K. K. Frese, G. Denicola, C. Feig, C. Combs, S. P. Winter, H. Ireland-Zecchini, S. Reichelt, W. J. Howat, A. Chang, M. Dhara, L. Wang, F. Ruckert, R. Grutzmann, C. Pilarsky, K. Izeradjene, S. R. Hingorani, P. Huang, S. E. Davies, W. Plunkett, M. Egorin, R. H. Hruban, N. Whitebread, K. McGovern, J. Adams, C. Iacobuzio-Donahue, J. Griffiths, and D. A. Tuveson. 2009. Inhibition of Hedgehog signaling enhances delivery of chemotherapy in a mouse model of pancreatic cancer. Science 324:1457-1461.

128. Tian, H., C. A. Callahan, K. J. DuPree, W. C. Darbonne, C. P. Ahn, S. J. Scales, and F. J. de Sauvage. 2009. Hedgehog signaling is restricted to the stromal compartment during pancreatic carcinogenesis. Proc Natl Acad Sci U S A 106:4254-4259.

129. Lee, J. J., R. M. Perera, H. Wang, D. C. Wu, X. S. Liu, S. Han, J. Fitamant, P. D. Jones, K. S. Ghanta, S. Kawano, J. M. Nagle, V. Deshpande, Y. Boucher, T. Kato, J. K. Chen, J. K. Willmann, N. Bardeesy, and P. A. Beachy. 2014. Stromal response to Hedgehog signaling restrains pancreatic cancer progression. Proc Natl Acad Sci U S A 111:E3091E3100.

130. Rajurkar, M., W. E. De Jesus-Monge, D. R. Driscoll, V. A. Appleman, H. Huang, J. L. Cotton, D. S. Klimstra, L. J. Zhu, K. Simin, L. Xu, A. P. McMahon, B. C. Lewis, and J. Mao. 2012. The activity of Gli transcription factors is essential for Kras-induced pancreatic tumorigenesis. Proc Natl Acad Sci U S A 109:E1038-E1047.

131. Mills, L. D., Y. Zhang, R. J. Marler, M. Herreros-Villanueva, L. Zhang, L. L. Almada, F. Couch, C. Wetmore, M. M. Pasca di, and M. E. Fernandez-Zapico. 2013. Loss of the transcription factor GLI1 identifies a signaling network in the tumor microenvironment mediating KRAS oncogene-induced transformation. J Biol Chem 288:11786-11794.

132. Morris, J. P., S. C. Wang, and M. Hebrok. 2010. KRAS, Hedgehog, Wnt and the twisted developmental biology of pancreatic ductal adenocarcinoma. Nat Rev Cancer 10:683695.

133. Meier, F., B. Schittek, S. Busch, C. Garbe, K. Smalley, K. Satyamoorthy, G. Li, and M. Herlyn. 2005. The RAS/RAF/MEK/ERK and PI3K/AKT signaling pathways present molecular targets for the effective treatment of advanced melanoma. Front Biosci 10:2986-3001. 
134. Beck, B. and C. Blanpain. 2013. Unravelling cancer stem cell potential. Nat Rev Cancer 13:727-738.

135. O'Brien, C. A., A. Kreso, and C. H. Jamieson. 2010. Cancer stem cells and self-renewal. Clin Cancer Res 16:3113-3120.

136. Kreso, A. and J. E. Dick. 2014. Evolution of the cancer stem cell model. Cell Stem Cell 14:275-291.

137. Trosko, J. E. 2009. Review paper: cancer stem cells and cancer nonstem cells: from adult stem cells or from reprogramming of differentiated somatic cells. Vet Pathol 46:176-193.

138. Gupta, P. B., C. M. Fillmore, G. Jiang, S. D. Shapira, K. Tao, C. Kuperwasser, and E. S. Lander. 2011. Stochastic state transitions give rise to phenotypic equilibrium in populations of cancer cells. Cell 146:633-644.

139. Ghiaur, G., J. Gerber, and R. J. Jones. 2012. Concise review: Cancer stem cells and minimal residual disease. Stem Cells 30:89-93.

140. Clevers, H. 2011. The cancer stem cell: premises, promises and challenges. Nat Med 17:313-319.

141. Quintana, E., M. Shackleton, M. S. Sabel, D. R. Fullen, T. M. Johnson, and S. J. Morrison. 2008. Efficient tumour formation by single human melanoma cells. Nature 456:593-598.

142. Zhou, B. B., H. Zhang, M. Damelin, K. G. Geles, J. C. Grindley, and P. B. Dirks. 2009. Tumour-initiating cells: challenges and opportunities for anticancer drug discovery. Nat Rev Drug Discov 8:806-823.

143. Li, X., M. T. Lewis, J. Huang, C. Gutierrez, C. K. Osborne, M. F. Wu, S. G. Hilsenbeck, A. Pavlick, X. Zhang, G. C. Chamness, H. Wong, J. Rosen, and J. C. Chang. 2008. Intrinsic resistance of tumorigenic breast cancer cells to chemotherapy. J Natl Cancer Inst 100:672-679.

144. Bao, S., Q. Wu, R. E. McLendon, Y. Hao, Q. Shi, A. B. Hjelmeland, M. W. Dewhirst, D. D. Bigner, and J. N. Rich. 2006. Glioma stem cells promote radioresistance by preferential activation of the DNA damage response. Nature 444:756-760. 
145. Dawood, S., L. Austin, and M. Cristofanilli. 2014. Cancer stem cells: implications for cancer therapy. Oncology (Williston. Park) 28:1101-7, 1110.

146. Kim, J. K., H. Y. Jeon, and H. Kim. 2015. The molecular mechanisms underlying the therapeutic resistance of cancer stem cells. Arch Pharm Res 38:389-401.

147. DI, C. and Y. Zhao. 2015. Multiple drug resistance due to resistance to stem cells and stem cell treatment progress in cancer (Review). Exp Ther Med 9:289-293.

148. Pece, S., D. Tosoni, S. Confalonieri, G. Mazzarol, M. Vecchi, S. Ronzoni, L. Bernard, G. Viale, P. G. Pelicci, and P. P. Di Fiore. 2010. Biological and molecular heterogeneity of breast cancers correlates with their cancer stem cell content. Cell 140:62-73.

149. Balic, M., H. Lin, L. Young, D. Hawes, A. Giuliano, G. McNamara, R. H. Datar, and R. J. Cote. 2006. Most early disseminated cancer cells detected in bone marrow of breast cancer patients have a putative breast cancer stem cell phenotype. Clin Cancer Res 12:5615-5621.

150. Hermann, P. C., S. L. Huber, T. Herrler, A. Aicher, J. W. Ellwart, M. Guba, C. J. Bruns, and C. Heeschen. 2007. Distinct populations of cancer stem cells determine tumor growth and metastatic activity in human pancreatic cancer. Cell Stem Cell 1:313-323.

151. Baccelli, I. and A. Trumpp. 2012. The evolving concept of cancer and metastasis stem cells. J Cell Biol 198:281-293.

152. Chen, K., Y. H. Huang, and J. L. Chen. 2013. Understanding and targeting cancer stem cells: therapeutic implications and challenges. Acta Pharmacol Sin. 34:732-740.

153. Pattabiraman, D. R. and R. A. Weinberg. 2014. Tackling the cancer stem cells - what challenges do they pose? Nat Rev Drug Discov 13:497-512.

154. Merchant, A. A. and W. Matsui. 2010. Targeting Hedgehog--a cancer stem cell pathway. Clin Cancer Res 16:3130-3140.

155. Justilien, V. and A. P. Fields. 2015. Molecular pathways: novel approaches for improved therapeutic targeting of Hedgehog signaling in cancer stem cells. Clin Cancer Res 21:505-513. 
156. Clement, V., P. Sanchez, T. N. de, I. Radovanovic, and A. Altaba. 2007. HEDGEHOGGLI1 signaling regulates human glioma growth, cancer stem cell self-renewal, and tumorigenicity. Curr Biol 17:165-172.

157. Ulasov, I. V., S. Nandi, M. Dey, A. M. Sonabend, and M. S. Lesniak. 2011. Inhibition of Sonic hedgehog and Notch pathways enhances sensitivity of CD133(+) glioma stem cells to temozolomide therapy. Mol Med 17:103-112.

158. Sarangi, A., J. G. Valadez, S. Rush, T. W. Abel, R. C. Thompson, and M. K. Cooper. 2009. Targeted inhibition of the Hedgehog pathway in established malignant glioma xenografts enhances survival. Oncogene 28:3468-3476.

159. Takezaki, T., T. Hide, H. Takanaga, H. Nakamura, J. Kuratsu, and T. Kondo. 2011. Essential role of the Hedgehog signaling pathway in human glioma-initiating cells. Cancer Sci 102:1306-1312.

160. Yan, G. N., L. Yang, Y. F. Lv, Y. Shi, L. L. Shen, X. H. Yao, Q. N. Guo, P. Zhang, Y. H. Cui, X. Zhang, X. W. Bian, and D. Y. Guo. 2014. Endothelial cells promote stem-like phenotype of glioma cells through activating the Hedgehog pathway. J Pathol 234:11-22.

161. Varnat, F., A. Duquet, M. Malerba, M. Zbinden, C. Mas, P. Gervaz, and A. Altaba. 2009. Human colon cancer epithelial cells harbour active HEDGEHOG-GLI signalling that is essential for tumour growth, recurrence, metastasis and stem cell survival and expansion. EMBO Mol Med 1:338-351.

162. Song, Z., W. Yue, B. Wei, N. Wang, T. Li, L. Guan, S. Shi, Q. Zeng, X. Pei, and L. Chen. 2011. Sonic hedgehog pathway is essential for maintenance of cancer stem-like cells in human gastric cancer. PLoS One 6:e17687.

163. Santini, R., M. C. Vinci, S. Pandolfi, J. Y. Penachioni, V. Montagnani, B. Olivito, R. Gattai, N. Pimpinelli, G. Gerlini, L. Borgognoni, and B. Stecca. 2012. Hedgehog-GLI signaling drives self-renewal and tumorigenicity of human melanoma-initiating cells. Stem Cells 30:1808-1818.

164. Dierks, C., R. Beigi, G. R. Guo, K. Zirlik, M. R. Stegert, P. Manley, C. Trussell, A. Schmitt-Graeff, K. Landwerlin, H. Veelken, and M. Warmuth. 2008. Expansion of BcrAbl-positive leukemic stem cells is dependent on Hedgehog pathway activation. Cancer Cell 14:238-249. 
165. Peacock, C. D., Q. Wang, G. S. Gesell, I. M. Corcoran-Schwartz, E. Jones, J. Kim, W. L. Devereux, J. T. Rhodes, C. A. Huff, P. A. Beachy, D. N. Watkins, and W. Matsui. 2007. Hedgehog signaling maintains a tumor stem cell compartment in multiple myeloma. Proc Natl Acad Sci U S A 104:4048-4053.

166. Watkins, D. N., D. M. Berman, S. G. Burkholder, B. Wang, P. A. Beachy, and S. B. Baylin. 2003. Hedgehog signalling within airway epithelial progenitors and in small-cell lung cancer. Nature 422:313-317.

167. Liu, S., G. Dontu, I. D. Mantle, S. Patel, N. S. Ahn, K. W. Jackson, P. Suri, and M. S. Wicha. 2006. Hedgehog signaling and Bmi-1 regulate self-renewal of normal and malignant human mammary stem cells. Cancer Res 66:6063-6071.

168. Goel, H. L., B. Pursell, C. Chang, L. M. Shaw, J. Mao, K. Simin, P. Kumar, C. W. Vander Kooi, L. D. Shultz, D. L. Greiner, J. H. Norum, R. Toftgard, C. Kuperwasser, and A. M. Mercurio. 2013. GLI1 regulates a novel neuropilin-2/alpha6beta1 integrin based autocrine pathway that contributes to breast cancer initiation. EMBO Mol Med 5:488508.

169. Justilien, V., M. P. Walsh, S. A. Ali, E. A. Thompson, N. R. Murray, and A. P. Fields. 2014. The PRKCI and SOX2 oncogenes are coamplified and cooperate to activate Hedgehog signaling in lung squamous cell carcinoma. Cancer Cell 25:139-151.

170. Huang, F. T., Y. X. Zhuan-Sun, Y. Y. Zhuang, S. L. Wei, J. Tang, W. B. Chen, and S. N. Zhang. 2012. Inhibition of hedgehog signaling depresses self-renewal of pancreatic cancer stem cells and reverses chemoresistance. Int J Oncol 41:1707-1714.

171. Singh, S., D. Chitkara, R. Mehrazin, S. W. Behrman, R. W. Wake, and R. I. Mahato. 2012. Chemoresistance in prostate cancer cells is regulated by miRNAs and Hedgehog pathway. PLoS One 7:e40021.

172. Steg, A. D., A. A. Katre, K. S. Bevis, A. Ziebarth, Z. C. Dobbin, M. M. Shah, R. D. Alvarez, and C. N. Landen. 2012. Smoothened antagonists reverse taxane resistance in ovarian cancer. Mol Cancer Ther 11:1587-1597.

173. Yoon, C., d. J. Park, B. Schmidt, N. J. Thomas, H. J. Lee, T. S. Kim, Y. Y. Janjigian, D. J. Cohen, and S. S. Yoon. 2014. CD44 expression denotes a subpopulation of gastric cancer cells in which Hedgehog signaling promotes chemotherapy resistance. Clin Cancer Res 20:3974-3988. 
174. Sims-Mourtada, J., J. G. Izzo, J. Ajani, and K. S. Chao. 2007. Sonic Hedgehog promotes multiple drug resistance by regulation of drug transport. Oncogene 26:5674-5679.

175. Heretsch, P., L. Tzagkaroulaki, and A. Giannis. 2010. Modulators of the hedgehog signaling pathway. Bioorg Med Chem 18:6613-6624.

176. Ng, J. M. and T. Curran. 2011. The Hedgehog's tale: developing strategies for targeting cancer. Nat Rev Cancer 11:493-501.

177. Xie, J., C. M. Bartels, S. W. Barton, and D. Gu. 2013. Targeting hedgehog signaling in cancer: research and clinical developments. Onco. Targets Ther 6:1425-1435.

178. Lin, T. L. and W. Matsui. 2012. Hedgehog pathway as a drug target: Smoothened inhibitors in development. Onco. Targets Ther 5:47-58.

179. Chen, J. K., J. Taipale, M. K. Cooper, and P. A. Beachy. 2002. Inhibition of Hedgehog signaling by direct binding of cyclopamine to Smoothened. Genes Dev 16:2743-2748.

180. Aszterbaum, M., J. Epstein, A. Oro, V. Douglas, P. E. LeBoit, M. P. Scott, and E. H. Epstein, Jr. 1999. Ultraviolet and ionizing radiation enhance the growth of BCCs and trichoblastomas in patched heterozygous knockout mice. Nat Med 5:1285-1291.

181. Oro, A. E., K. M. Higgins, Z. Hu, J. M. Bonifas, E. H. Epstein, Jr., and M. P. Scott. 1997. Basal cell carcinomas in mice overexpressing sonic hedgehog. Science 276:817-821.

182. Grachtchouk, M., R. Mo, S. Yu, X. Zhang, H. Sasaki, C. C. Hui, and A. A. Dlugosz. 2000. Basal cell carcinomas in mice overexpressing Gli2 in skin. Nat Genet 24:216-217.

183. Athar, M., C. Li, X. Tang, S. Chi, X. Zhang, A. L. Kim, S. K. Tyring, L. Kopelovich, J. Hebert, E. H. Epstein, Jr., D. R. Bickers, and J. Xie. 2004. Inhibition of smoothened signaling prevents ultraviolet B-induced basal cell carcinomas through regulation of Fas expression and apoptosis. Cancer Res 64:7545-7552.

184. Lipinski, R. J., P. R. Hutson, P. W. Hannam, R. J. Nydza, I. M. Washington, R. W. Moore, G. G. Girdaukas, R. E. Peterson, and W. Bushman. 2008. Dose- and routedependent teratogenicity, toxicity, and pharmacokinetic profiles of the hedgehog signaling antagonist cyclopamine in the mouse. Toxicol Sci 104:189-197. 
185. Mahindroo, N., C. Punchihewa, and N. Fujii. 2009. Hedgehog-Gli signaling pathway inhibitors as anticancer agents. J Med Chem 52:3829-3845.

186. Tabs, S. and O. Avci. 2004. Induction of the differentiation and apoptosis of tumor cells in vivo with efficiency and selectivity. Eur J Dermatol 14:96-102.

187. Tang, T., J. Y. Tang, D. Li, M. Reich, C. A. Callahan, L. Fu, R. L. Yauch, F. Wang, K. Kotkow, K. S. Chang, E. Shpall, A. Wu, L. L. Rubin, J. C. Marsters, Jr., E. H. Epstein, Jr., I. Caro, and F. J. de Sauvage. 2011. Targeting superficial or nodular Basal cell carcinoma with topically formulated small molecule inhibitor of smoothened. Clin Cancer Res 17:3378-3387.

188. Von Hoff, D. D., P. M. LoRusso, C. M. Rudin, J. C. Reddy, R. L. Yauch, R. Tibes, G. J. Weiss, M. J. Borad, C. L. Hann, J. R. Brahmer, H. M. Mackey, B. L. Lum, W. C. Darbonne, J. C. Marsters, Jr., F. J. de Sauvage, and J. A. Low. 2009. Inhibition of the hedgehog pathway in advanced basal-cell carcinoma. N Engl J Med 361:1164-1172.

189. LoRusso, P. M., C. M. Rudin, J. C. Reddy, R. Tibes, G. J. Weiss, M. J. Borad, C. L. Hann, J. R. Brahmer, I. Chang, W. C. Darbonne, R. A. Graham, K. L. Zerivitz, J. A. Low, and D. D. Von Hoff. 2011. Phase I trial of hedgehog pathway inhibitor vismodegib (GDC-0449) in patients with refractory, locally advanced or metastatic solid tumors. Clin Cancer Res 17:2502-2511.

190. Sekulic, A., M. R. Migden, A. E. Oro, L. Dirix, K. D. Lewis, J. D. Hainsworth, J. A. Solomon, S. Yoo, S. T. Arron, P. A. Friedlander, E. Marmur, C. M. Rudin, A. L. Chang, J. A. Low, H. M. Mackey, R. L. Yauch, R. A. Graham, J. C. Reddy, and A. Hauschild. 2012. Efficacy and safety of vismodegib in advanced basal-cell carcinoma. N Engl J Med 366:2171-2179.

191. Tang, J. Y., J. M. kay-Wiggan, M. Aszterbaum, R. L. Yauch, J. Lindgren, K. Chang, C. Coppola, A. M. Chanana, J. Marji, D. R. Bickers, and E. H. Epstein, Jr. 2012. Inhibiting the hedgehog pathway in patients with the basal-cell nevus syndrome. N Engl J Med 366:2180-2188.

192. Axelson, M., K. Liu, X. Jiang, K. He, J. Wang, H. Zhao, D. Kufrin, T. Palmby, Z. Dong, A. M. Russell, S. Miksinski, P. Keegan, and R. Pazdur. 2013. U.S. Food and Drug Administration approval: vismodegib for recurrent, locally advanced, or metastatic basal cell carcinoma. Clin Cancer Res 19:2289-2293.

193. Athar, M., C. Li, A. L. Kim, V. S. Spiegelman, and D. R. Bickers. 2014. Sonic hedgehog signaling in Basal cell nevus syndrome. Cancer Res 74:4967-4975. 
194. Yauch, R. L., G. J. Dijkgraaf, B. Alicke, T. Januario, C. P. Ahn, T. Holcomb, K. Pujara, J. Stinson, C. A. Callahan, T. Tang, J. F. Bazan, Z. Kan, S. Seshagiri, C. L. Hann, S. E. Gould, J. A. Low, C. M. Rudin, and F. J. de Sauvage. 2009. Smoothened mutation confers resistance to a Hedgehog pathway inhibitor in medulloblastoma. Science 326:572-574.

195. Yang, Z. J., T. Ellis, S. L. Markant, T. A. Read, J. D. Kessler, M. Bourboulas, U. Schuller, R. Machold, G. Fishell, D. H. Rowitch, B. J. Wainwright, and R. J. WechslerReya. 2008. Medulloblastoma can be initiated by deletion of Patched in lineage-restricted progenitors or stem cells. Cancer Cell 14:135-145.

196. Lee, M. J., B. A. Hatton, E. H. Villavicencio, P. C. Khanna, S. D. Friedman, S. Ditzler, B. Pullar, K. Robison, K. F. White, C. Tunkey, M. LeBlanc, J. Randolph-Habecker, S. E. Knoblaugh, S. Hansen, A. Richards, B. J. Wainwright, K. McGovern, and J. M. Olson. 2012. Hedgehog pathway inhibitor saridegib (IPI-926) increases lifespan in a mouse medulloblastoma model. Proc Natl Acad Sci U S A 109:7859-7864.

197. Rohner, A., M. E. Spilker, J. L. Lam, B. Pascual, D. Bartkowski, Q. J. Li, A. H. Yang, G. Stevens, M. Xu, P. A. Wells, S. Planken, S. Nair, and S. Sun. 2012. Effective targeting of Hedgehog signaling in a medulloblastoma model with PF-5274857, a potent and selective Smoothened antagonist that penetrates the blood-brain barrier. Mol Cancer Ther 11:5765.

198. Berman, D. M., S. S. Karhadkar, A. R. Hallahan, J. I. Pritchard, C. G. Eberhart, D. N. Watkins, J. K. Chen, M. K. Cooper, J. Taipale, J. M. Olson, and P. A. Beachy. 2002. Medulloblastoma growth inhibition by hedgehog pathway blockade. Science 297:15591561.

199. Rudin, C. M., C. L. Hann, J. Laterra, R. L. Yauch, C. A. Callahan, L. Fu, T. Holcomb, J. Stinson, S. E. Gould, B. Coleman, P. M. LoRusso, D. D. Von Hoff, F. J. de Sauvage, and J. A. Low. 2009. Treatment of medulloblastoma with hedgehog pathway inhibitor GDC0449. N Engl J Med 361:1173-1178.

200. Lauth, M., A. Bergstrom, T. Shimokawa, and R. Toftgard. 2007. Inhibition of GLImediated transcription and tumor cell growth by small-molecule antagonists. Proc Natl Acad Sci U S A 104:8455-8460.

201. Hosoya, T., M. A. Arai, T. Koyano, T. Kowithayakorn, and M. Ishibashi. 2008. Naturally occurring small-molecule inhibitors of hedgehog/GLI-mediated transcription. Chembiochem 9:1082-1092. 
202. Infante, P., M. Mori, R. Alfonsi, F. Ghirga, F. Aiello, S. Toscano, C. Ingallina, M. Siler, D. Cucchi, A. Po, E. Miele, D. D'Amico, G. Canettieri, S. E. De, E. Ferretti, I. Screpanti, B. G. Uccello, M. Botta, B. Botta, A. Gulino, and M. L. Di. 2015. Gli1/DNA interaction is a druggable target for Hedgehog-dependent tumors. EMBO J 34:200-217.

203. Mahindroo, N., M. C. Connelly, C. Punchihewa, H. Kimura, M. P. Smeltzer, S. Wu, and N. Fujii. 2009. Structure-activity relationships and cancer-cell selective toxicity of novel inhibitors of glioma-associated oncogene homologue 1 (Gli1) mediated transcription. J Med Chem 52:4277-4287.

204. Epstein, E. H. 2008. Basal cell carcinomas: attack of the hedgehog. Nat Rev Cancer 8:743-754.

205. Stanton, B. Z., L. F. Peng, N. Maloof, K. Nakai, X. Wang, J. L. Duffner, K. M. Taveras, J. M. Hyman, S. W. Lee, A. N. Koehler, J. K. Chen, J. L. Fox, A. Mandinova, and S. L. Schreiber. 2009. A small molecule that binds Hedgehog and blocks its signaling in human cells. Nat Chem Biol 5:154-156.

206. Kim, J., J. Y. Tang, R. Gong, J. Kim, J. J. Lee, K. V. Clemons, C. R. Chong, K. S. Chang, M. Fereshteh, D. Gardner, T. Reya, J. O. Liu, E. H. Epstein, D. A. Stevens, and P. A. Beachy. 2010. Itraconazole, a commonly used antifungal that inhibits Hedgehog pathway activity and cancer growth. Cancer Cell 17:388-399.

207. Kim, D. J., J. Kim, K. Spaunhurst, J. Montoya, R. Khodosh, K. Chandra, T. Fu, A. Gilliam, M. Molgo, P. A. Beachy, and J. Y. Tang. 2014. Open-label, exploratory phase II trial of oral itraconazole for the treatment of basal cell carcinoma. J Clin Oncol 32:745751.

208. Kim, J., B. T. Aftab, J. Y. Tang, D. Kim, A. H. Lee, M. Rezaee, J. Kim, B. Chen, E. M. King, A. Borodovsky, G. J. Riggins, E. H. Epstein, Jr., P. A. Beachy, and C. M. Rudin. 2013. Itraconazole and arsenic trioxide inhibit Hedgehog pathway activation and tumor growth associated with acquired resistance to smoothened antagonists. Cancer Cell 23:23-34.

209. Beauchamp, E. M., L. Ringer, G. Bulut, K. P. Sajwan, M. D. Hall, Y. C. Lee, D. Peaceman, M. Ozdemirli, O. Rodriguez, T. J. Macdonald, C. Albanese, J. A. Toretsky, and A. Uren. 2011. Arsenic trioxide inhibits human cancer cell growth and tumor development in mice by blocking Hedgehog/GLI pathway. J Clin Invest 121:148-160. 
210. Tang, J. Y., T. Z. Xiao, Y. Oda, K. S. Chang, E. Shpall, A. Wu, P. L. So, J. Hebert, D. Bikle, and E. H. Epstein, Jr. 2011. Vitamin D3 inhibits hedgehog signaling and proliferation in murine Basal cell carcinomas. Cancer Prev Res (Phila) 4:744-751.

211. Uhmann, A., H. Niemann, B. Lammering, C. Henkel, I. Hess, F. Nitzki, A. Fritsch, N. Prufer, A. Rosenberger, C. Dullin, A. Schraepler, J. Reifenberger, S. Schweyer, T. Pietsch, F. Strutz, W. Schulz-Schaeffer, and H. Hahn. 2011. Antitumoral effects of calcitriol in basal cell carcinomas involve inhibition of hedgehog signaling and induction of vitamin D receptor signaling and differentiation. Mol Cancer Ther 10:2179-2188.

212. Berlin, J., J. C. Bendell, L. L. Hart, I. Firdaus, I. Gore, R. C. Hermann, M. F. Mulcahy, M. M. Zalupski, H. M. Mackey, R. L. Yauch, R. A. Graham, G. L. Bray, and J. A. Low. 2013. A randomized phase II trial of vismodegib versus placebo with FOLFOX or FOLFIRI and bevacizumab in patients with previously untreated metastatic colorectal cancer. Clin Cancer Res 19:258-267.

213. Kaye, S. B., L. Fehrenbacher, R. Holloway, A. Amit, B. Karlan, B. Slomovitz, P. Sabbatini, L. Fu, R. L. Yauch, I. Chang, and J. C. Reddy. 2012. A phase II, randomized, placebo-controlled study of vismodegib as maintenance therapy in patients with ovarian cancer in second or third complete remission. Clin Cancer Res 18:6509-6518.

214. Dellovade, T., J. T. Romer, T. Curran, and L. L. Rubin. 2006. The hedgehog pathway and neurological disorders. Annu Rev Neurosci 29:539-563.

215. Bhattacharyya, S., H. Yu, C. Mim, and A. Matouschek. 2014. Regulated protein turnover: snapshots of the proteasome in action. Nat Rev Mol Cell Biol 15:122-133.

216. Wilkinson, K. D. 2004. Ubiquitin: a Nobel protein. Cell 119:741-745.

217. Glickman, M. H. and A. Ciechanover. 2002. The ubiquitin-proteasome proteolytic pathway: destruction for the sake of construction. Physiol Rev 82:373-428.

218. Pickart, C. M. and M. J. Eddins. 2004. Ubiquitin: structures, functions, mechanisms. Biochim Biophys Acta 1695:55-72.

219. Hicke, L., H. L. Schubert, and C. P. Hill. 2005. Ubiquitin-binding domains. Nat Rev Mol Cell Biol 6:610-621. 
220. Dikic, I., S. Wakatsuki, and K. J. Walters. 2009. Ubiquitin-binding domains - from structures to functions. Nat Rev Mol Cell Biol 10:659-671.

221. Wang, X. and E. J. Terpstra. 2013. Ubiquitin receptors and protein quality control. J Mol Cell Cardiol. 55:73-84.

222. Nakayama, K. I. and K. Nakayama. 2006. Ubiquitin ligases: cell-cycle control and cancer. Nat Rev Cancer 6:369-381.

223. Voorhees, P. M., E. C. Dees, B. O'Neil, and R. Z. Orlowski. 2003. The proteasome as a target for cancer therapy. Clin Cancer Res 9:6316-6325.

224. Reinstein, E. and A. Ciechanover. 2006. Narrative review: protein degradation and human diseases: the ubiquitin connection. Ann Intern Med 145:676-684.

225. Richardson, P. G., P. Sonneveld, M. W. Schuster, D. Irwin, E. A. Stadtmauer, T. Facon, J. L. Harousseau, D. Ben-Yehuda, S. Lonial, H. Goldschmidt, D. Reece, J. F. SanMiguel, J. Blade, M. Boccadoro, J. Cavenagh, W. S. Dalton, A. L. Boral, D. L. Esseltine, J. B. Porter, D. Schenkein, K. C. Anderson, and Assessment of Proteasome Inhibition for Extending Remissions (APEX) Investigators. 2005. Bortezomib or high-dose dexamethasone for relapsed multiple myeloma. N Engl J Med 352:2487-2498.

226. Robak, T., H. Huang, J. Jin, J. Zhu, T. Liu, O. Samoilova, H. Pylypenko, G. Verhoef, N. Siritanaratkul, E. Osmanov, J. Alexeeva, J. Pereira, J. Drach, J. Mayer, X. Hong, R. Okamoto, L. Pei, B. Rooney, d. van, V, F. Cavalli, and LYM-3002 Investigators. 2015. Bortezomib-based therapy for newly diagnosed mantle-cell lymphoma. N Engl J Med 372:944-953.

227. Buac, D., M. Shen, S. Schmitt, F. R. Kona, R. Deshmukh, Z. Zhang, C. Neslund-Dudas, B. Mitra, and Q. P. Dou. 2013. From bortezomib to other inhibitors of the proteasome and beyond. Curr Pharm Des 19:4025-4038.

228. Okamoto, Y., T. Ozaki, K. Miyazaki, M. Aoyama, M. Miyazaki, and A. Nakagawara. 2003. UbcH10 is the cancer-related E2 ubiquitin-conjugating enzyme. Cancer Res 63:4167-4173.

229. Lau, A. W., H. Fukushima, and W. Wei. 2012. The Fbw7 and betaTRCP E3 ubiquitin ligases and their roles in tumorigenesis. Front Biosci (Landmark Ed) 17:2197-2212. 
230. Frescas, D. and M. Pagano. 2008. Deregulated proteolysis by the F-box proteins SKP2 and beta-TrCP: tipping the scales of cancer. Nat Rev Cancer 8:438-449.

231. Li, W., M. H. Bengtson, A. Ulbrich, A. Matsuda, V. A. Reddy, A. Orth, S. K. Chanda, S. Batalov, and C. A. Joazeiro. 2008. Genome-wide and functional annotation of human E3 ubiquitin ligases identifies MULAN, a mitochondrial E3 that regulates the organelle's dynamics and signaling. PLoS One 3:e1487.

232. van Wijk, S. J. and H. T. Timmers. 2010. The family of ubiquitin-conjugating enzymes (E2s): deciding between life and death of proteins. FASEB J 24:981-993.

233. Groettrup, M., C. Pelzer, G. Schmidtke, and K. Hofmann. 2008. Activating the ubiquitin family: UBA6 challenges the field. Trends Biochem Sci 33:230-237.

234. Schwob, E., T. Bohm, M. D. Mendenhall, and K. Nasmyth. 1994. The B-type cyclin kinase inhibitor p40SIC1 controls the G1 to S transition in S. cerevisiae. Cell 79:233-244.

235. Bai, C., P. Sen, K. Hofmann, L. Ma, M. Goebl, J. W. Harper, and S. J. Elledge. 1996. SKP1 connects cell cycle regulators to the ubiquitin proteolysis machinery through a novel motif, the F-box. Cell 86:263-274.

236. Mathias, N., S. L. Johnson, M. Winey, A. E. Adams, L. Goetsch, J. R. Pringle, B. Byers, and M. G. Goebl. 1996. Cdc53p acts in concert with Cdc4p and Cdc34p to control the G1-to-S-phase transition and identifies a conserved family of proteins. Mol Cell Biol 16:6634-6643.

237. Willems, A. R., S. Lanker, E. E. Patton, K. L. Craig, T. F. Nason, N. Mathias, R. Kobayashi, C. Wittenberg, and M. Tyers. 1996. Cdc53 targets phosphorylated G1 cyclins for degradation by the ubiquitin proteolytic pathway. Cell 86:453-463.

238. Skowyra, D., K. L. Craig, M. Tyers, S. J. Elledge, and J. W. Harper. 1997. F-box proteins are receptors that recruit phosphorylated substrates to the SCF ubiquitin-ligase complex. Cell 91:209-219.

239. Feldman, R. M., C. C. Correll, K. B. Kaplan, and R. J. Deshaies. 1997. A complex of Cdc4p, Skp1p, and Cdc53p/cullin catalyzes ubiquitination of the phosphorylated CDK inhibitor Sic1p. Cell 91:221-230. 
240. Ohta, T., J. J. Michel, A. J. Schottelius, and Y. Xiong. 1999. ROC1, a homolog of APC11, represents a family of cullin partners with an associated ubiquitin ligase activity. Mol Cell 3:535-541.

241. Kamura, T., D. M. Koepp, M. N. Conrad, D. Skowyra, R. J. Moreland, O. Iliopoulos, W. S. Lane, W. G. Kaelin, Jr., S. J. Elledge, R. C. Conaway, J. W. Harper, and J. W. Conaway. 1999. Rbx1, a component of the VHL tumor suppressor complex and SCF ubiquitin ligase. Science 284:657-661.

242. Skowyra, D., D. M. Koepp, T. Kamura, M. N. Conrad, R. C. Conaway, J. W. Conaway, S. J. Elledge, and J. W. Harper. 1999. Reconstitution of G1 cyclin ubiquitination with complexes containing SCFGrr1 and Rbx1. Science 284:662-665.

243. Petroski, M. D. and R. J. Deshaies. 2005. Function and regulation of cullin-RING ubiquitin ligases. Nat Rev Mol Cell Biol 6:9-20.

244. Deshaies, R. J. and C. A. Joazeiro. 2009. RING domain E3 ubiquitin ligases. Annu Rev Biochem 78:399-434.

245. Metzger, M. B., V. A. Hristova, and A. M. Weissman. 2012. HECT and RING finger families of E3 ubiquitin ligases at a glance. J Cell Sci 125:531-537.

246. Zhang, H., R. Kobayashi, K. Galaktionov, and D. Beach. 1995. p19Skp1 and p45Skp2 are essential elements of the cyclin A-CDK2 S phase kinase. Cell 82:915-925.

247. Stemmann, O. and J. Lechner. 1996. The Saccharomyces cerevisiae kinetochore contains a cyclin-CDK complexing homologue, as identified by in vitro reconstitution. EMBO J 15:3611-3620.

248. Yoshida, Y., A. Murakami, and K. Tanaka. 2011. Skp1 stabilizes the conformation of Fbox proteins. Biochem Biophys Res Commun 410:24-28.

249. Schulman, B. A., A. C. Carrano, P. D. Jeffrey, Z. Bowen, E. R. Kinnucan, M. S. Finnin, S. J. Elledge, J. W. Harper, M. Pagano, and N. P. Pavletich. 2000. Insights into SCF ubiquitin ligases from the structure of the Skp1-Skp2 complex. Nature 408:381-386.

250. Kipreos, E. T., L. E. Lander, J. P. Wing, W. W. He, and E. M. Hedgecock. 1996. cul-1 is required for cell cycle exit in C. elegans and identifies a novel gene family. Cell 85:829839. 
251. Chen, Z., J. Sui, F. Zhang, and C. Zhang. 2015. Cullin family proteins and tumorigenesis: genetic association and molecular mechanisms. J Cancer 6:233-242.

252. Yu, Z. K., J. L. Gervais, and H. Zhang. 1998. Human CUL-1 associates with the SKP1/SKP2 complex and regulates $\mathrm{p} 21(\mathrm{CIP} 1 / \mathrm{WAF} 1)$ and cyclin D proteins. Proc Natl Acad Sci U S A 95:11324-11329.

253. Yu, H., J. M. Peters, R. W. King, A. M. Page, P. Hieter, and M. W. Kirschner. 1998. Identification of a cullin homology region in a subunit of the anaphase-promoting complex. Science 279:1219-1222.

254. Kamitani, T., K. Kito, H. P. Nguyen, and E. T. Yeh. 1997. Characterization of NEDD8, a developmentally down-regulated ubiquitin-like protein. J Biol Chem 272:28557-28562.

255. Gong, L. and E. T. Yeh. 1999. Identification of the activating and conjugating enzymes of the NEDD8 conjugation pathway. J Biol Chem 274:12036-12042.

256. Furukawa, M., Y. Zhang, J. McCarville, T. Ohta, and Y. Xiong. 2000. The CUL1 Cterminal sequence and ROC1 are required for efficient nuclear accumulation, NEDD8 modification, and ubiquitin ligase activity of CUL1. Mol Cell Biol 20:8185-8197.

257. Read, M. A., J. E. Brownell, T. B. Gladysheva, M. Hottelet, L. A. Parent, M. B. Coggins, J. W. Pierce, V. N. Podust, R. S. Luo, V. Chau, and V. J. Palombella. 2000. Nedd8 modification of cul-1 activates $\mathrm{SCF}($ beta(TrCP))-dependent ubiquitination of IkappaBalpha. Mol Cell Biol 20:2326-2333.

258. Zheng, J., X. Yang, J. M. Harrell, S. Ryzhikov, E. H. Shim, K. Lykke-Andersen, N. Wei, H. Sun, R. Kobayashi, and H. Zhang. 2002. CAND1 binds to unneddylated CUL1 and regulates the formation of SCF ubiquitin E3 ligase complex. Mol Cell 10:1519-1526.

259. Liu, J., M. Furukawa, T. Matsumoto, and Y. Xiong. 2002. NEDD8 modification of CUL1 dissociates p120(CAND1), an inhibitor of CUL1-SKP1 binding and SCF ligases. Mol Cell 10:1511-1518.

260. Goldenberg, S. J., T. C. Cascio, S. D. Shumway, K. C. Garbutt, J. Liu, Y. Xiong, and N. Zheng. 2004. Structure of the Cand1-Cul1-Roc1 complex reveals regulatory mechanisms for the assembly of the multisubunit cullin-dependent ubiquitin ligases. Cell 119:517528. 
261. Zheng, N., B. A. Schulman, L. Song, J. J. Miller, P. D. Jeffrey, P. Wang, C. Chu, D. M. Koepp, S. J. Elledge, M. Pagano, R. C. Conaway, J. W. Conaway, J. W. Harper, and N. P. Pavletich. 2002. Structure of the Cul1-Rbx1-Skp1-F boxSkp2 SCF ubiquitin ligase complex. Nature 416:703-709.

262. Freemont, P. S., I. M. Hanson, and J. Trowsdale. 1991. A novel cysteine-rich sequence motif. Cell 64:483-484.

263. Lovering, R., I. M. Hanson, K. L. Borden, S. Martin, N. J. O'Reilly, G. I. Evan, D. Rahman, D. J. Pappin, J. Trowsdale, and P. S. Freemont. 1993. Identification and preliminary characterization of a protein motif related to the zinc finger. Proc Natl Acad Sci U S A 90:2112-2116.

264. Deshaies, R. J. 1999. SCF and Cullin/Ring H2-based ubiquitin ligases. Annu Rev Cell Dev Biol 15:435-467.

265. Freemont, P. S. 2000. RING for destruction? Curr Biol 10:R84-R87.

266. Moynihan, T. P., H. C. Ardley, U. Nuber, S. A. Rose, P. F. Jones, A. F. Markham, M. Scheffner, and P. A. Robinson. 1999. The ubiquitin-conjugating enzymes $\mathrm{UbcH} 7$ and UbcH8 interact with RING finger/IBR motif-containing domains of HHARI and H7AP1. J Biol Chem 274:30963-30968.

267. Duda, D. M., J. L. Olszewski, J. P. Schuermann, I. Kurinov, D. J. Miller, A. Nourse, A. F. Alpi, and B. A. Schulman. 2013. Structure of HHARI, a RING-IBR-RING ubiquitin ligase: autoinhibition of an Ariadne-family E3 and insights into ligation mechanism. Structure 21:1030-1041.

268. Moynihan, T. P., H. C. Ardley, J. P. Leek, J. Thompson, N. S. Brindle, A. F. Markham, and P. A. Robinson. 1996. Characterization of a human ubiquitin-conjugating enzyme gene UBE2L3. Mamm. Genome 7:520-525.

269. Kumar, A. and J. V. Paietta. 1995. The sulfur controller-2 negative regulatory gene of Neurospora crassa encodes a protein with beta-transducin repeats. Proc Natl Acad Sci U S A 92:3343-3347.

270. Kipreos, E. T. and M. Pagano. 2000. The F-box protein family. Genome Biol 1:REVIEWS3002. 
271. Skaar, J. R., J. K. Pagan, and M. Pagano. 2013. Mechanisms and function of substrate recruitment by F-box proteins. Nat Rev Mol Cell Biol 14:369-381.

272. Cenciarelli, C., D. S. Chiaur, D. Guardavaccaro, W. Parks, M. Vidal, and M. Pagano. 1999. Identification of a family of human F-box proteins. Curr Biol 9:1177-1179.

273. Winston, J. T., D. M. Koepp, C. Zhu, S. J. Elledge, and J. W. Harper. 1999. A family of mammalian F-box proteins. Curr Biol 9:1180-1182.

274. Yumimoto, K., T. Muneoka, T. Tsuboi, and K. I. Nakayama. 2013. Substrate binding promotes formation of the Skp1-Cul1-Fbxl3 ( $\mathrm{SCF}(\mathrm{Fbxl3})$ ) protein complex. J Biol Chem 288:32766-32776.

275. Fong, H. K., J. B. Hurley, R. S. Hopkins, R. Miake-Lye, M. S. Johnson, R. F. Doolittle, and M. I. Simon. 1986. Repetitive segmental structure of the transducin beta subunit: homology with the CDC4 gene and identification of related mRNAs. Proc Natl Acad Sci U S A 83:2162-2166.

276. Fuchs, S. Y., V. S. Spiegelman, and K. G. Kumar. 2004. The many faces of beta-TrCP E3 ubiquitin ligases: reflections in the magic mirror of cancer. Oncogene 23:2028-2036.

277. Suzuki, H., T. Chiba, T. Suzuki, T. Fujita, T. Ikenoue, M. Omata, K. Furuichi, H. Shikama, and K. Tanaka. 2000. Homodimer of two F-box proteins betaTrCP1 or betaTrCP2 binds to IkappaBalpha for signal-dependent ubiquitination. J Biol Chem 275:2877-2884.

278. Guardavaccaro, D., Y. Kudo, J. Boulaire, M. Barchi, L. Busino, M. Donzelli, F. Margottin-Goguet, P. K. Jackson, L. Yamasaki, and M. Pagano. 2003. Control of meiotic and mitotic progression by the F box protein beta-Trcp1 in vivo. Dev Cell 4:799-812.

279. Nakayama, K., S. Hatakeyama, S. Maruyama, A. Kikuchi, K. Onoe, R. A. Good, and K. I. Nakayama. 2003. Impaired degradation of inhibitory subunit of NF-kappa B (I kappa B) and beta-catenin as a result of targeted disruption of the beta-TrCP1 gene. Proc Natl Acad Sci U S A 100:8752-8757.

280. Low, T. Y., M. Peng, R. Magliozzi, S. Mohammed, D. Guardavaccaro, and A. J. Heck. 2014. A systems-wide screen identifies substrates of the SCFbetaTrCP ubiquitin ligase. Sci Signal 7:rs8. 
281. Kim, T. Y., P. F. Siesser, K. L. Rossman, D. Goldfarb, K. Mackinnon, F. Yan, X. Yi, M. J. MacCoss, R. T. Moon, C. J. Der, and M. B. Major. 2015. Substrate trapping proteomics reveals targets of the betaTrCP2/FBXW11 ubiquitin ligase. Mol Cell Biol $35: 167-181$.

282. Wu, G., G. Xu, B. A. Schulman, P. D. Jeffrey, J. W. Harper, and N. P. Pavletich. 2003. Structure of a beta-TrCP1-Skp1-beta-catenin complex: destruction motif binding and lysine specificity of the SCF(beta-TrCP1) ubiquitin ligase. Mol Cell 11:1445-1456.

283. Kim, M. O., S. H. Kim, Y. Y. Cho, J. Nadas, C. H. Jeong, K. Yao, D. J. Kim, D. H. Yu, Y. S. Keum, K. Y. Lee, Z. Huang, A. M. Bode, and Z. Dong. 2012. ERK1 and ERK2 regulate embryonic stem cell self-renewal through phosphorylation of Klf4. Nat Struct Mol Biol 19:283-290.

284. Skaar, J. R., V. D'Angiolella, J. K. Pagan, and M. Pagano. 2009. SnapShot: F Box Proteins II. Cell 137:1358, 1358.

285. Davis, M., A. Hatzubai, J. S. Andersen, E. Ben-Shushan, G. Z. Fisher, A. Yaron, A. Bauskin, F. Mercurio, M. Mann, and Y. Ben-Neriah. 2002. Pseudosubstrate regulation of the SCF(beta-TrCP) ubiquitin ligase by hnRNP-U. Genes Dev 16:439-451.

286. Busino, L., M. Donzelli, M. Chiesa, D. Guardavaccaro, D. Ganoth, N. V. Dorrello, A. Hershko, M. Pagano, and G. F. Draetta. 2003. Degradation of Cdc25A by beta-TrCP during $\mathrm{S}$ phase and in response to DNA damage. Nature 426:87-91.

287. Kanemori, Y., K. Uto, and N. Sagata. 2005. Beta-TrCP recognizes a previously undescribed nonphosphorylated destruction motif in Cdc25A and Cdc25B phosphatases. Proc Natl Acad Sci U S A 102:6279-6284.

288. Watanabe, N., H. Arai, Y. Nishihara, M. Taniguchi, N. Watanabe, T. Hunter, and H. Osada. 2004. M-phase kinases induce phospho-dependent ubiquitination of somatic Wee1 by SCFbeta-TrCP. Proc Natl Acad Sci U S A 101:4419-4424.

289. Mailand, N., S. Bekker-Jensen, J. Bartek, and J. Lukas. 2006. Destruction of Claspin by SCFbetaTrCP restrains Chk1 activation and facilitates recovery from genotoxic stress. Mol Cell 23:307-318.

290. Peschiaroli, A., N. V. Dorrello, D. Guardavaccaro, M. Venere, T. Halazonetis, N. E. Sherman, and M. Pagano. 2006. SCFbetaTrCP-mediated degradation of Claspin regulates recovery from the DNA replication checkpoint response. Mol Cell 23:319-329. 
291. Yaron, A., A. Hatzubai, M. Davis, I. Lavon, S. Amit, A. M. Manning, J. S. Andersen, M. Mann, F. Mercurio, and Y. Ben-Neriah. 1998. Identification of the receptor component of the IkappaBalpha-ubiquitin ligase. Nature 396:590-594.

292. Tan, P., S. Y. Fuchs, A. Chen, K. Wu, C. Gomez, Z. Ronai, and Z. Q. Pan. 1999. Recruitment of a ROC1-CUL1 ubiquitin ligase by Skp1 and HOS to catalyze the ubiquitination of I kappa B alpha. Mol Cell 3:527-533.

293. Wu, C. and S. Ghosh. 1999. beta-TrCP mediates the signal-induced ubiquitination of IkappaBbeta. J Biol Chem 274:29591-29594.

294. Spencer, E., J. Jiang, and Z. J. Chen. 1999. Signal-induced ubiquitination of IkappaBalpha by the F-box protein Slimb/beta-TrCP. Genes Dev 13:284-294.

295. Shirane, M., S. Hatakeyama, K. Hattori, K. Nakayama, and K. Nakayama. 1999. Common pathway for the ubiquitination of IkappaBalpha, IkappaBbeta, and IkappaBepsilon mediated by the F-box protein FWD1. J Biol Chem 274:28169-28174.

296. Kroll, M., F. Margottin, A. Kohl, P. Renard, H. Durand, J. P. Concordet, F. Bachelerie, F. renzana-Seisdedos, and R. Benarous. 1999. Inducible degradation of IkappaBalpha by the proteasome requires interaction with the F-box protein h-betaTrCP. J Biol Chem 274:7941-7945.

297. Hatakeyama, S., M. Kitagawa, K. Nakayama, M. Shirane, M. Matsumoto, K. Hattori, H. Higashi, H. Nakano, K. Okumura, K. Onoe, R. A. Good, and K. Nakayama. 1999. Ubiquitin-dependent degradation of IkappaBalpha is mediated by a ubiquitin ligase Skp1/Cul 1/F-box protein FWD1. Proc Natl Acad Sci U S A 96:3859-3863.

298. Winston, J. T., P. Strack, P. Beer-Romero, C. Y. Chu, S. J. Elledge, and J. W. Harper. 1999. The SCFbeta-TRCP-ubiquitin ligase complex associates specifically with phosphorylated destruction motifs in IkappaBalpha and beta-catenin and stimulates IkappaBalpha ubiquitination in vitro. Genes Dev 13:270-283.

299. Karin, M. and F. R. Greten. 2005. NF-kappaB: linking inflammation and immunity to cancer development and progression. Nat Rev Immunol 5:749-759.

300. Muerkoster, S., A. Arlt, B. Sipos, M. Witt, M. Grossmann, G. Kloppel, H. Kalthoff, U. R. Folsch, and H. Schafer. 2005. Increased expression of the E3-ubiquitin ligase receptor subunit betaTRCP1 relates to constitutive nuclear factor-kappaB activation and chemoresistance in pancreatic carcinoma cells. Cancer Res 65:1316-1324. 
301. Spiegelman, V. S., W. Tang, A. M. Chan, M. Igarashi, S. A. Aaronson, D. A. Sassoon, M. Katoh, T. J. Slaga, and S. Y. Fuchs. 2002. Induction of homologue of Slimb ubiquitin ligase receptor by mitogen signaling. J Biol Chem 277:36624-36630.

302. Ougolkov, A., B. Zhang, K. Yamashita, V. Bilim, M. Mai, S. Y. Fuchs, and T. Minamoto. 2004. Associations among beta-TrCP, an E3 ubiquitin ligase receptor, betacatenin, and NF-kappaB in colorectal cancer. J Natl Cancer Inst 96:1161-1170.

303. Dhawan, P. and A. Richmond. 2002. A novel NF-kappa B-inducing kinase-MAPK signaling pathway up-regulates NF-kappa B activity in melanoma cells. J Biol Chem 277:7920-7928.

304. Liu, J., K. G. Suresh Kumar, D. Yu, S. A. Molton, M. McMahon, M. Herlyn, A. ThomasTikhonenko, and S. Y. Fuchs. 2007. Oncogenic BRAF regulates beta-Trcp expression and NF-kappaB activity in human melanoma cells. Oncogene 26:1954-1958.

305. Koch, A., A. Waha, W. Hartmann, A. Hrychyk, U. Schuller, A. Waha, K. A. Wharton, Jr., S. Y. Fuchs, S. D. von, and T. Pietsch. 2005. Elevated expression of Wnt antagonists is a common event in hepatoblastomas. Clin Cancer Res 11:4295-4304.

306. Kudo, Y., D. Guardavaccaro, P. G. Santamaria, R. Koyama-Nasu, E. Latres, R. Bronson, L. Yamasaki, and M. Pagano. 2004. Role of F-box protein betaTrcp1 in mammary gland development and tumorigenesis. Mol Cell Biol 24:8184-8194.

307. Dorrello, N. V., A. Peschiaroli, D. Guardavaccaro, N. H. Colburn, N. E. Sherman, and M. Pagano. 2006. S6K1- and betaTRCP-mediated degradation of PDCD4 promotes protein translation and cell growth. Science 314:467-471.

308. Yang, H. S., A. P. Jansen, A. A. Komar, X. Zheng, W. C. Merrick, S. Costes, S. J. Lockett, N. Sonenberg, and N. H. Colburn. 2003. The transformation suppressor Pdcd4 is a novel eukaryotic translation initiation factor $4 \mathrm{~A}$ binding protein that inhibits translation. Mol Cell Biol 23:26-37.

309. MacDonald, B. T., K. Tamai, and X. He. 2009. Wnt/beta-catenin signaling: components, mechanisms, and diseases. Dev Cell 17:9-26.

310. Clevers, H. and R. Nusse. 2012. Wnt/beta-catenin signaling and disease. Cell 149:11921205. 
311. Aberle, H., A. Bauer, J. Stappert, A. Kispert, and R. Kemler. 1997. beta-catenin is a target for the ubiquitin-proteasome pathway. EMBO J 16:3797-3804.

312. Liu, C., Y. Kato, Z. Zhang, V. M. Do, B. A. Yankner, and X. He. 1999. beta-Trcp couples beta-catenin phosphorylation-degradation and regulates Xenopus axis formation. Proc Natl Acad Sci U S A 96:6273-6278.

313. Liu, C., Y. Li, M. Semenov, C. Han, G. H. Baeg, Y. Tan, Z. Zhang, X. Lin, and X. He. 2002. Control of beta-catenin phosphorylation/degradation by a dual-kinase mechanism. Cell 108:837-847.

314. Amit, S., A. Hatzubai, Y. Birman, J. S. Andersen, E. Ben-Shushan, M. Mann, Y. BenNeriah, and I. Alkalay. 2002. Axin-mediated CKI phosphorylation of beta-catenin at Ser 45: a molecular switch for the Wnt pathway. Genes Dev 16:1066-1076.

315. Yanagawa, S., Y. Matsuda, J. S. Lee, H. Matsubayashi, S. Sese, T. Kadowaki, and A. Ishimoto. 2002. Casein kinase I phosphorylates the Armadillo protein and induces its degradation in Drosophila. EMBO J 21:1733-1742.

316. Polakis, P. 2007. The many ways of Wnt in cancer. Curr Opin Genet Dev 17:45-51.

317. Kalluri, R. and R. A. Weinberg. 2009. The basics of epithelial-mesenchymal transition. J Clin Invest 119:1420-1428.

318. Yook, J. I., X. Y. Li, I. Ota, E. R. Fearon, and S. J. Weiss. 2005. Wnt-dependent regulation of the E-cadherin repressor snail. J Biol Chem 280:11740-11748.

319. Zhou, B. P., J. Deng, W. Xia, J. Xu, Y. M. Li, M. Gunduz, and M. C. Hung. 2004. Dual regulation of Snail by GSK-3beta-mediated phosphorylation in control of epithelialmesenchymal transition. Nat Cell Biol 6:931-940.

320. Honda, R., H. Tanaka, and H. Yasuda. 1997. Oncoprotein MDM2 is a ubiquitin ligase E3 for tumor suppressor p53. FEBS Lett 420:25-27.

321. Inuzuka, H., A. Tseng, D. Gao, B. Zhai, Q. Zhang, S. Shaik, L. Wan, X. L. Ang, C. Mock, H. Yin, J. M. Stommel, S. Gygi, G. Lahav, J. Asara, Z. X. Xiao, W. G. Kaelin, Jr., J. W. Harper, and W. Wei. 2010. Phosphorylation by casein kinase I promotes the turnover of the Mdm2 oncoprotein via the SCF(beta-TRCP) ubiquitin ligase. Cancer Cell 18:147-159. 
322. Chen, Y., N. Gallaher, R. H. Goodman, and S. M. Smolik. 1998. Protein kinase A directly regulates the activity and proteolysis of cubitus interruptus. Proc Natl Acad Sci U S A 95:2349-2354.

323. Jia, J., K. Amanai, G. Wang, J. Tang, B. Wang, and J. Jiang. 2002. Shaggy/GSK3 antagonizes Hedgehog signalling by regulating Cubitus interruptus. Nature 416:548-552.

324. Price, M. A. and D. Kalderon. 2002. Proteolysis of the Hedgehog signaling effector Cubitus interruptus requires phosphorylation by Glycogen Synthase Kinase 3 and Casein Kinase 1. Cell 108:823-835.

325. McConnell, B. B. and V. W. Yang. 2010. Mammalian Kruppel-like factors in health and diseases. Physiol Rev 90:1337-1381.

326. Brey, C. W., M. P. Nelder, T. Hailemariam, R. Gaugler, and S. Hashmi. 2009. Kruppellike family of transcription factors: an emerging new frontier in fat biology. Int J Biol Sci 5:622-636.

327. Kadonaga, J. T., K. R. Carner, F. R. Masiarz, and R. Tjian. 1987. Isolation of cDNA encoding transcription factor $\mathrm{Sp} 1$ and functional analysis of the DNA binding domain. Cell 51:1079-1090.

328. Pearson, R., J. Fleetwood, S. Eaton, M. Crossley, and S. Bao. 2008. Kruppel-like transcription factors: a functional family. Int J Biochem Cell Biol 40:1996-2001.

329. Turner, J. and M. Crossley. 1998. Cloning and characterization of mCtBP2, a corepressor that associates with basic Kruppel-like factor and other mammalian transcriptional regulators. EMBO J 17:5129-5140.

330. van Vliet, J., J. Turner, and M. Crossley. 2000. Human Kruppel-like factor 8: a CACCCbox binding protein that associates with $\mathrm{CtBP}$ and represses transcription. Nucleic Acids Res 28:1955-1962.

331. Schuierer, M., K. Hilger-Eversheim, T. Dobner, A. K. Bosserhoff, M. Moser, J. Turner, M. Crossley, and R. Buettner. 2001. Induction of AP-2alpha expression by adenoviral infection involves inactivation of the AP-2rep transcriptional corepressor CtBP1. J Biol Chem 276:27944-27949. 
332. Miller, J., A. D. McLachlan, and A. Klug. 1985. Repetitive zinc-binding domains in the protein transcription factor IIIA from Xenopus oocytes. EMBO J 4:1609-1614.

333. Dang, D. T., J. Pevsner, and V. W. Yang. 2000. The biology of the mammalian Kruppellike family of transcription factors. Int J Biochem Cell Biol 32:1103-1121.

334. Miller, I. J. and J. J. Bieker. 1993. A novel, erythroid cell-specific murine transcription factor that binds to the CACCC element and is related to the Kruppel family of nuclear proteins. Mol Cell Biol 13:2776-2786.

335. Shields, J. M. and V. W. Yang. 1998. Identification of the DNA sequence that interacts with the gut-enriched Kruppel-like factor. Nucleic Acids Res 26:796-802.

336. Shields, J. M., R. J. Christy, and V. W. Yang. 1996. Identification and characterization of a gene encoding a gut-enriched Kruppel-like factor expressed during growth arrest. J Biol Chem 271:20009-20017.

337. Garrett-Sinha, L. A., H. Eberspaecher, M. F. Seldin, and C. B. de. 1996. A gene for a novel zinc-finger protein expressed in differentiated epithelial cells and transiently in certain mesenchymal cells. J Biol Chem 271:31384-31390.

338. Yet, S. F., M. M. McA'Nulty, S. C. Folta, H. W. Yen, M. Yoshizumi, C. M. Hsieh, M. D. Layne, M. T. Chin, H. Wang, M. A. Perrella, M. K. Jain, and M. E. Lee. 1998. Human EZF, a Kruppel-like zinc finger protein, is expressed in vascular endothelial cells and contains transcriptional activation and repression domains. J Biol Chem 273:1026-1031.

339. Chiambaretta, F., G. F. De, G. Turet, G. Marceau, P. Gain, B. Dastugue, D. Rigal, and V. Sapin. 2004. Cell and tissue specific expression of human Kruppel-like transcription factors in human ocular surface. Mol Vis 10:901-909.

340. Cullingford, T. E., M. J. Butler, A. K. Marshall, e. L. Tham, P. H. Sugden, and A. Clerk. 2008. Differential regulation of Kruppel-like factor family transcription factor expression in neonatal rat cardiac myocytes: effects of endothelin-1, oxidative stress and cytokines. Biochim Biophys Acta 1783:1229-1236.

341. Fruman, D. A., G. Z. Ferl, S. S. An, A. C. Donahue, A. B. Satterthwaite, and O. N. Witte. 2002. Phosphoinositide 3-kinase and Bruton's tyrosine kinase regulate overlapping sets of genes in B lymphocytes. Proc Natl Acad Sci U S A 99:359-364. 
342. Panigada, M., S. Porcellini, F. Sutti, L. Doneda, O. Pozzoli, G. G. Consalez, M. Guttinger, and F. Grassi. 1999. GKLF in thymus epithelium as a developmentally regulated element of thymocyte-stroma cross-talk. Mech Dev 81:103-113.

343. Rechsteiner, M. and S. W. Rogers. 1996. PEST sequences and regulation by proteolysis. Trends Biochem Sci 21:267-271.

344. Geiman, D. E., H. Ton-That, J. M. Johnson, and V. W. Yang. 2000. Transactivation and growth suppression by the gut-enriched Kruppel-like factor (Kruppel-like factor 4) are dependent on acidic amino acid residues and protein-protein interaction. Nucleic Acids Res 28:1106-1113.

345. Roth, S. Y., J. M. Denu, and C. D. Allis. 2001. Histone acetyltransferases. Annu Rev Biochem 70:81-120.

346. Ai, W., H. Zheng, X. Yang, Y. Liu, and T. C. Wang. 2007. Tip60 functions as a potential corepressor of KLF4 in regulation of HDC promoter activity. Nucleic Acids Res 35:6137-6149.

347. Feinberg, M. W., Z. Cao, A. K. Wara, M. A. Lebedeva, S. Senbanerjee, and M. K. Jain. 2005. Kruppel-like factor 4 is a mediator of proinflammatory signaling in macrophages. J Biol Chem 280:38247-38258.

348. Dang, D. T., W. Zhao, C. S. Mahatan, D. E. Geiman, and V. W. Yang. 2002. Opposing effects of Kruppel-like factor 4 (gut-enriched Kruppel-like factor) and Kruppel-like factor 5 (intestinal-enriched Kruppel-like factor) on the promoter of the Kruppel-like factor 4 gene. Nucleic Acids Res 30:2736-2741.

349. Yori, J. L., E. Johnson, G. Zhou, M. K. Jain, and R. A. Keri. 2010. Kruppel-like factor 4 inhibits epithelial-to-mesenchymal transition through regulation of E-cadherin gene expression. J Biol Chem 285:16854-16863.

350. Luo, A., J. Kong, G. Hu, C. C. Liew, M. Xiong, X. Wang, J. Ji, T. Wang, H. Zhi, M. Wu, and Z. Liu. 2004. Discovery of Ca2+-relevant and differentiation-associated genes downregulated in esophageal squamous cell carcinoma using cDNA microarray. Oncogene 23:1291-1299.

351. Brembeck, F. H. and A. K. Rustgi. 2000. The tissue-dependent keratin 19 gene transcription is regulated by GKLF/KLF4 and Sp1. J Biol Chem 275:28230-28239. 
352. Hinnebusch, B. F., A. Siddique, J. W. Henderson, M. S. Malo, W. Zhang, C. P. Athaide, M. A. Abedrapo, X. Chen, V. W. Yang, and R. A. Hodin. 2004. Enterocyte differentiation marker intestinal alkaline phosphatase is a target gene of the gut-enriched Kruppel-like factor. Am J Physiol Gastrointest Liver Physiol 286:G23-G30.

353. Siddique, A., M. S. Malo, L. M. Ocuin, B. F. Hinnebusch, M. A. Abedrapo, J. W. Henderson, W. Zhang, M. Mozumder, V. W. Yang, and R. A. Hodin. 2003. Convergence of the thyroid hormone and gut-enriched Kruppel-like factor pathways in the context of enterocyte differentiation. J Gastrointest Surg 7:1053-1061.

354. Zeng, Y., S. Zhuang, J. Gloddek, C. C. Tseng, G. R. Boss, and R. B. Pilz. 2006. Regulation of cGMP-dependent protein kinase expression by Rho and Kruppel-like transcription factor-4. J Biol Chem 281:16951-16961.

355. Miller, K. A., E. A. Eklund, M. L. Peddinghaus, Z. Cao, N. Fernandes, P. W. Turk, B. Thimmapaya, and S. A. Weitzman. 2001. Kruppel-like factor 4 regulates laminin alpha 3A expression in mammary epithelial cells. J Biol Chem 276:42863-42868.

356. Higaki, Y., D. Schullery, Y. Kawata, M. Shnyreva, C. Abrass, and K. Bomsztyk. 2002. Synergistic activation of the rat laminin gammal chain promoter by the gut-enriched Kruppel-like factor (GKLF/KLF4) and Sp1. Nucleic Acids Res 30:2270-2279.

357. Chew, Y. C., G. Adhikary, W. Xu, G. M. Wilson, and R. L. Eckert. 2013. Protein kinase $\mathrm{C}$ delta increases Kruppel-like factor 4 protein, which drives involucrin gene transcription in differentiating keratinocytes. J Biol Chem 288:17759-17768.

358. Nakatake, Y., N. Fukui, Y. Iwamatsu, S. Masui, K. Takahashi, R. Yagi, K. Yagi, J. Miyazaki, R. Matoba, M. S. Ko, and H. Niwa. 2006. Klf4 cooperates with Oct3/4 and Sox 2 to activate the Lefty1 core promoter in embryonic stem cells. Mol Cell Biol 26:7772-7782.

359. Jiang, J., Y. S. Chan, Y. H. Loh, J. Cai, G. Q. Tong, C. A. Lim, P. Robson, S. Zhong, and H. H. Ng. 2008. A core Klf circuitry regulates self-renewal of embryonic stem cells. Nat Cell Biol 10:353-360.

360. Zhang, W., D. E. Geiman, J. M. Shields, D. T. Dang, C. S. Mahatan, K. H. Kaestner, J. R. Biggs, A. S. Kraft, and V. W. Yang. 2000. The gut-enriched Kruppel-like factor (Kruppel-like factor 4) mediates the transactivating effect of p53 on the p21WAF1/Cip1 promoter. J Biol Chem 275:18391-18398. 
361. Nickenig, G., S. Baudler, C. Muller, C. Werner, N. Werner, H. Welzel, K. Strehlow, and M. Bohm. 2002. Redox-sensitive vascular smooth muscle cell proliferation is mediated by GKLF and Id3 in vitro and in vivo. FASEB J 16:1077-1086.

362. Chen, X., E. M. Whitney, S. Y. Gao, and V. W. Yang. 2003. Transcriptional profiling of Kruppel-like factor 4 reveals a function in cell cycle regulation and epithelial differentiation. J Mol Biol 326:665-677.

363. Lin, C. C., L. Z. Liu, J. B. Addison, W. F. Wonderlin, A. V. Ivanov, and J. M. Ruppert. 2011. A KLF4-miRNA-206 autoregulatory feedback loop can promote or inhibit protein translation depending upon cell context. Mol Cell Biol 31:2513-2527.

364. Moon, J. S., H. E. Kim, E. Koh, S. H. Park, W. J. Jin, B. W. Park, S. W. Park, and K. S. Kim. 2011. Kruppel-like factor 4 (KLF4) activates the transcription of the gene for the platelet isoform of phosphofructokinase (PFKP) in breast cancer. J Biol Chem 286:23808-23816.

365. Wang, H., L. Yang, M. S. Jamaluddin, and D. D. Boyd. 2004. The Kruppel-like KLF4 transcription factor, a novel regulator of urokinase receptor expression, drives synthesis of this binding site in colonic crypt luminal surface epithelial cells. J Biol Chem 279:22674-22683.

366. Kanai, M., D. Wei, Q. Li, Z. Jia, J. Ajani, X. Le, J. Yao, and K. Xie. 2006. Loss of Kruppel-like factor 4 expression contributes to $\mathrm{Sp} 1$ overexpression and human gastric cancer development and progression. Clin Cancer Res 12:6395-6402.

367. Zhang, W., J. M. Shields, K. Sogawa, Y. Fujii-Kuriyama, and V. W. Yang. 1998. The gut-enriched Kruppel-like factor suppresses the activity of the CYP1A1 promoter in an Sp1-dependent fashion. J Biol Chem 273:17917-17925.

368. Noti, J. D., A. K. Johnson, and J. D. Dillon. 2005. The leukocyte integrin gene CD11d is repressed by gut-enriched Kruppel-like factor 4 in myeloid cells. J Biol Chem 280:34493457.

369. Evans, P. M., W. Zhang, X. Chen, J. Yang, K. K. Bhakat, and C. Liu. 2007. Kruppel-like factor 4 is acetylated by p300 and regulates gene transcription via modulation of histone acetylation. J Biol Chem 282:33994-34002.

370. Wei, X., H. Xu, and D. Kufe. 2007. Human mucin 1 oncoprotein represses transcription of the p53 tumor suppressor gene. Cancer Res 67:1853-1858. 
371. Zhang, W., X. Chen, Y. Kato, P. M. Evans, S. Yuan, J. Yang, P. G. Rychahou, V. W. Yang, X. He, B. M. Evers, and C. Liu. 2006. Novel cross talk of Kruppel-like factor 4 and beta-catenin regulates normal intestinal homeostasis and tumor repression. Mol Cell Biol 26:2055-2064.

372. Ghaleb, A. M., J. P. Katz, K. H. Kaestner, J. X. Du, and V. W. Yang. 2007. Kruppel-like factor 4 exhibits antiapoptotic activity following gamma-radiation-induced DNA damage. Oncogene 26:2365-2373.

373. Shie, J. L., Z. Y. Chen, M. J. O'Brien, R. G. Pestell, M. E. Lee, and C. C. Tseng. 2000. Role of gut-enriched Kruppel-like factor in colonic cell growth and differentiation. Am J Physiol Gastrointest Liver Physiol 279:G806-G814.

374. Klaewsongkram, J., Y. Yang, S. Golech, J. Katz, K. H. Kaestner, and N. P. Weng. 2007. Kruppel-like factor 4 regulates B cell number and activation-induced B cell proliferation. J Immunol 179:4679-4684.

375. Yoon, H. S., A. M. Ghaleb, M. O. Nandan, I. M. Hisamuddin, W. B. Dalton, and V. W. Yang. 2005. Kruppel-like factor 4 prevents centrosome amplification following gammairradiation-induced DNA damage. Oncogene 24:4017-4025.

376. Shi, M., J. Cui, J. Du, D. Wei, Z. Jia, J. Zhang, Z. Zhu, Y. Gao, and K. Xie. 2014. A novel KLF4/LDHA signaling pathway regulates aerobic glycolysis in and progression of pancreatic cancer. Clin Cancer Res 20:4370-4380.

377. Chen, Z. Y., J. L. Shie, and C. C. Tseng. 2002. Gut-enriched Kruppel-like factor represses ornithine decarboxylase gene expression and functions as checkpoint regulator in colonic cancer cells. J Biol Chem 277:46831-46839.

378. Adam, P. J., C. P. Regan, M. B. Hautmann, and G. K. Owens. 2000. Positive- and negative-acting Kruppel-like transcription factors bind a transforming growth factor beta control element required for expression of the smooth muscle cell differentiation marker SM22alpha in vivo. J Biol Chem 275:37798-37806.

379. Liu, Y., S. Sinha, and G. Owens. 2003. A transforming growth factor-beta control element required for SM alpha-actin expression in vivo also partially mediates GKLFdependent transcriptional repression. J Biol Chem 278:48004-48011. 
380. Tiwari, N., N. Meyer-Schaller, P. Arnold, H. Antoniadis, M. Pachkov, N. E. van, and G. Christofori. 2013. Klf4 is a transcriptional regulator of genes critical for EMT, including Jnk1 (Mapk8). PLoS One 8:e57329.

381. Li, D., Z. Peng, H. Tang, P. Wei, X. Kong, D. Yan, F. Huang, Q. Li, X. Le, Q. Li, and K. Xie. 2011. KLF4-mediated negative regulation of IFITM3 expression plays a critical role in colon cancer pathogenesis. Clin Cancer Res 17:3558-3568.

382. Chen, X., D. C. Johns, D. E. Geiman, E. Marban, D. T. Dang, G. Hamlin, R. Sun, and V. W. Yang. 2001. Kruppel-like factor 4 (gut-enriched Kruppel-like factor) inhibits cell proliferation by blocking G1/S progression of the cell cycle. J Biol Chem 276:3042330428.

383. Harper, J. W., G. R. Adami, N. Wei, K. Keyomarsi, and S. J. Elledge. 1993. The p21 Cdk-interacting protein Cip1 is a potent inhibitor of G1 cyclin-dependent kinases. Cell 75:805-816.

384. Matsuoka, S., M. C. Edwards, C. Bai, S. Parker, P. Zhang, A. Baldini, J. W. Harper, and S. J. Elledge. 1995. p57KIP2, a structurally distinct member of the p21CIP1 Cdk inhibitor family, is a candidate tumor suppressor gene. Genes Dev 9:650-662.

385. Kato, M., A. Ishizaki, U. Hellman, C. Wernstedt, M. Kyogoku, K. Miyazono, C. H. Heldin, and K. Funa. 1995. A human keratinocyte cell line produces two autocrine growth inhibitors, transforming growth factor-beta and insulin-like growth factor binding protein-6, in a calcium- and cell density-dependent manner. J Biol Chem 270:1237312379.

386. Hermeking, H., C. Lengauer, K. Polyak, T. C. He, L. Zhang, S. Thiagalingam, K. W. Kinzler, and B. Vogelstein. 1997. 14-3-3 sigma is a p53-regulated inhibitor of G2/M progression. Mol Cell 1:3-11.

387. Whitney, E. M., A. M. Ghaleb, X. Chen, and V. W. Yang. 2006. Transcriptional profiling of the cell cycle checkpoint gene kruppel-like factor 4 reveals a global inhibitory function in macromolecular biosynthesis. Gene Expr 13:85-96.

388. Segre, J. A., C. Bauer, and E. Fuchs. 1999. Klf4 is a transcription factor required for establishing the barrier function of the skin. Nat Genet 22:356-360.

389. Hardman, M. J., P. Sisi, D. N. Banbury, and C. Byrne. 1998. Patterned acquisition of skin barrier function during development. Development 125:1541-1552. 
390. Chew, Y. C., G. Adhikary, G. M. Wilson, E. A. Reece, and R. L. Eckert. 2011. Protein kinase $\mathrm{C}$ (PKC) delta suppresses keratinocyte proliferation by increasing p21(Cip1) level by a KLF4 transcription factor-dependent mechanism. J Biol Chem 286:28772-28782.

391. Katz, J. P., N. Perreault, B. G. Goldstein, C. S. Lee, P. A. Labosky, V. W. Yang, and K. H. Kaestner. 2002. The zinc-finger transcription factor Klf4 is required for terminal differentiation of goblet cells in the colon. Development 129:2619-2628.

392. Ghaleb, A. M., B. B. McConnell, K. H. Kaestner, and V. W. Yang. 2011. Altered intestinal epithelial homeostasis in mice with intestine-specific deletion of the Kruppellike factor 4 gene. Dev Biol 349:310-320.

393. Zheng, H., D. M. Pritchard, X. Yang, E. Bennett, G. Liu, C. Liu, and W. Ai. 2009. KLF4 gene expression is inhibited by the notch signaling pathway that controls goblet cell differentiation in mouse gastrointestinal tract. Am J Physiol Gastrointest Liver Physiol 296:G490-G498.

394. Real, P. J., V. Tosello, T. Palomero, M. Castillo, E. Hernando, S. E. de, M. L. Sulis, K. Barnes, C. Sawai, I. Homminga, J. Meijerink, I. Aifantis, G. Basso, C. Cordon-Cardo, W. $\mathrm{Ai}$, and A. Ferrando. 2009. Gamma-secretase inhibitors reverse glucocorticoid resistance in $\mathrm{T}$ cell acute lymphoblastic leukemia. Nat Med 15:50-58.

395. Ghaleb, A. M., G. Aggarwal, A. B. Bialkowska, M. O. Nandan, and V. W. Yang. 2008. Notch inhibits expression of the Kruppel-like factor 4 tumor suppressor in the intestinal epithelium. Mol Cancer Res 6:1920-1927.

396. Donohoe, D. R., N. Garge, X. Zhang, W. Sun, T. M. O'Connell, M. K. Bunger, and S. J. Bultman. 2011. The microbiome and butyrate regulate energy metabolism and autophagy in the mammalian colon. Cell Metab 13:517-526.

397. Chen, Z. Y., S. Rex, and C. C. Tseng. 2004. Kruppel-like factor 4 is transactivated by butyrate in colon cancer cells. J Nutr 134:792-798.

398. Flandez, M., S. Guilmeau, P. Blache, and L. H. Augenlicht. 2008. KLF4 regulation in intestinal epithelial cell maturation. Exp Cell Res 314:3712-3723.

399. Jenkins, T. D., O. G. Opitz, J. Okano, and A. K. Rustgi. 1998. Transactivation of the human keratin 4 and Epstein-Barr virus ED-L2 promoters by gut-enriched Kruppel-like factor. J Biol Chem 273:10747-10754. 
400. Tetreault, M. P., Y. Yang, J. Travis, Q. C. Yu, A. Klein-Szanto, J. W. Tobias, and J. P. Katz. 2010. Esophageal squamous cell dysplasia and delayed differentiation with deletion of kruppel-like factor 4 in murine esophagus. Gastroenterology 139:171-181.

401. Katz, J. P., N. Perreault, B. G. Goldstein, L. Actman, S. R. McNally, D. G. Silberg, E. E. Furth, and K. H. Kaestner. 2005. Loss of Klf4 in mice causes altered proliferation and differentiation and precancerous changes in the adult stomach. Gastroenterology 128:935-945.

402. Zhang, P., P. Basu, L. C. Redmond, P. E. Morris, J. W. Rupon, G. D. Ginder, and J. A. Lloyd. 2005. A functional screen for Kruppel-like factors that regulate the human gamma-globin gene through the CACCC promoter element. Blood Cells Mol Dis 35:227235.

403. van Zelm, M. C., B. M. van der, R. D. de, B. H. Barendregt, E. F. de Haas, M. J. Reinders, A. C. Lankester, T. Revesz, F. J. Staal, and J. J. van Dongen. 2005. Ig gene rearrangement steps are initiated in early human precursor B cell subsets and correlate with specific transcription factor expression. J Immunol 175:5912-5922.

404. Good, K. L. and S. G. Tangye. 2007. Decreased expression of Kruppel-like factors in memory B cells induces the rapid response typical of secondary antibody responses. Proc Natl Acad Sci U S A 104:13420-13425.

405. Park, C. S., P. H. Lee, T. Yamada, A. Burns, Y. Shen, M. Puppi, and H. D. Lacorazza. 2012. Kruppel-like factor 4 (KLF4) promotes the survival of natural killer cells and maintains the number of conventional dendritic cells in the spleen. J Leukoc. Biol 91:739-750.

406. Alder, J. K., R. W. Georgantas, III, R. L. Hildreth, I. M. Kaplan, S. Morisot, X. Yu, M. McDevitt, and C. I. Civin. 2008. Kruppel-like factor 4 is essential for inflammatory monocyte differentiation in vivo. J Immunol 180:5645-5652.

407. Feinberg, M. W., A. K. Wara, Z. Cao, M. A. Lebedeva, F. Rosenbauer, H. Iwasaki, H. Hirai, J. P. Katz, R. L. Haspel, S. Gray, K. Akashi, J. Segre, K. H. Kaestner, D. G. Tenen, and M. K. Jain. 2007. The Kruppel-like factor KLF4 is a critical regulator of monocyte differentiation. EMBO J 26:4138-4148.

408. Hu, B., Z. Wu, T. Liu, M. R. Ullenbruch, H. Jin, and S. H. Phan. 2007. Gut-enriched Kruppel-like factor interaction with Smad3 inhibits myofibroblast differentiation. Am J Respir. Cell Mol Biol 36:78-84. 
409. Birsoy, K., Z. Chen, and J. Friedman. 2008. Transcriptional regulation of adipogenesis by KLF4. Cell Metab 7:339-347.

410. Green, H. and M. Meuth. 1974. An established pre-adipose cell line and its differentiation in culture. Cell 3:127-133.

411. Rosen, E. D. and O. A. MacDougald. 2006. Adipocyte differentiation from the inside out. Nat Rev Mol Cell Biol 7:885-896.

412. Moore, D. L., M. G. Blackmore, Y. Hu, K. H. Kaestner, J. L. Bixby, V. P. Lemmon, and J. L. Goldberg. 2009. KLF family members regulate intrinsic axon regeneration ability. Science 326:298-301.

413. Apara, A. and J. L. Goldberg. 2014. Molecular mechanisms of the suppression of axon regeneration by KLF transcription factors. Neural Regen. Res 9:1418-1421.

414. Qin, S., Y. Zou, and C. L. Zhang. 2013. Cross-talk between KLF4 and STAT3 regulates axon regeneration. Nat Commun 4:2633.

415. Qin, S. and C. L. Zhang. 2012. Role of Kruppel-like factor 4 in neurogenesis and radial neuronal migration in the developing cerebral cortex. Mol Cell Biol 32:4297-4305.

416. Lundin, C., M. North, K. Erixon, K. Walters, D. Jenssen, A. S. Goldman, and T. Helleday. 2005. Methyl methanesulfonate (MMS) produces heat-labile DNA damage but no detectable in vivo DNA double-strand breaks. Nucleic Acids Res 33:3799-3811.

417. Yoon, H. S., X. Chen, and V. W. Yang. 2003. Kruppel-like factor 4 mediates p53dependent G1/S cell cycle arrest in response to DNA damage. J Biol Chem 278:21012105.

418. Zhou, Q., Y. Hong, Q. Zhan, Y. Shen, and Z. Liu. 2009. Role for Kruppel-like factor 4 in determining the outcome of p53 response to DNA damage. Cancer Res 69:8284-8292.

419. Methe, H., M. Balcells, M. C. Alegret, M. Santacana, B. Molins, A. Hamik, M. K. Jain, and E. R. Edelman. 2007. Vascular bed origin dictates flow pattern regulation of endothelial adhesion molecule expression. Am J Physiol Heart Circ Physiol 292:H2167$\mathrm{H} 2175$. 
420. Hamik, A., Z. Lin, A. Kumar, M. Balcells, S. Sinha, J. Katz, M. W. Feinberg, R. E. Gerzsten, E. R. Edelman, and M. K. Jain. 2007. Kruppel-like factor 4 regulates endothelial inflammation. J Biol Chem 282:13769-13779.

421. Kee, H. J. and H. Kook. 2009. Kruppel-like factor 4 mediates histone deacetylase inhibitor-induced prevention of cardiac hypertrophy. J Mol Cell Cardiol. 47:770-780.

422. Salmon, M., W. F. Johnston, A. Woo, N. H. Pope, G. Su, G. R. Upchurch, Jr., G. K. Owens, and G. Ailawadi. 2013. KLF4 regulates abdominal aortic aneurysm morphology and deletion attenuates aneurysm formation. Circulation 128:S163-S174.

423. Yoshida, T., M. Yamashita, C. Horimai, and M. Hayashi. 2014. Deletion of Kruppel-like factor 4 in endothelial and hematopoietic cells enhances neointimal formation following vascular injury. J Am Heart Assoc 3:e000622.

424. Yoshida, T., Q. Gan, and G. K. Owens. 2008. Kruppel-like factor 4, Elk-1, and histone deacetylases cooperatively suppress smooth muscle cell differentiation markers in response to oxidized phospholipids. Am J Physiol Cell Physiol 295:C1175-C1182.

425. Yoshida, T., K. H. Kaestner, and G. K. Owens. 2008. Conditional deletion of Kruppellike factor 4 delays downregulation of smooth muscle cell differentiation markers but accelerates neointimal formation following vascular injury. Circ Res 102:1548-1557.

426. Kuhn, A. R., K. Schlauch, R. Lao, A. J. Halayko, W. T. Gerthoffer, and C. A. Singer. 2010. MicroRNA expression in human airway smooth muscle cells: role of miR-25 in regulation of airway smooth muscle phenotype. Am J Respir. Cell Mol Biol 42:506-513.

427. Ray, A., M. Alalem, and B. K. Ray. 2013. Loss of epigenetic Kruppel-like factor 4 histone deacetylase (KLF-4-HDAC)-mediated transcriptional suppression is crucial in increasing vascular endothelial growth factor (VEGF) expression in breast cancer. J Biol Chem 288:27232-27242.

428. Tetreault, M. P., M. L. Wang, Y. Yang, J. Travis, Q. C. Yu, A. J. Klein-Szanto, and J. P. Katz. 2010. Klf4 overexpression activates epithelial cytokines and inflammationmediated esophageal squamous cell cancer in mice. Gastroenterology 139:2124-2134.

429. Classen, A., J. Lloberas, and A. Celada. 2009. Macrophage activation: classical versus alternative. Methods Mol Biol 531:29-43. 
430. Das, H., A. Kumar, Z. Lin, W. D. Patino, P. M. Hwang, M. W. Feinberg, P. K. Majumder, and M. K. Jain. 2006. Kruppel-like factor 2 (KLF2) regulates proinflammatory activation of monocytes. Proc Natl Acad Sci U S A 103:6653-6658.

431. Nishikawa, S., R. A. Goldstein, and C. R. Nierras. 2008. The promise of human induced pluripotent stem cells for research and therapy. Nat Rev Mol Cell Biol 9:725-729.

432. Takahashi, K. and S. Yamanaka. 2006. Induction of pluripotent stem cells from mouse embryonic and adult fibroblast cultures by defined factors. Cell 126:663-676.

433. Takahashi, K., K. Tanabe, M. Ohnuki, M. Narita, T. Ichisaka, K. Tomoda, and S. Yamanaka. 2007. Induction of pluripotent stem cells from adult human fibroblasts by defined factors. Cell 131:861-872.

434. Wernig, M., A. Meissner, R. Foreman, T. Brambrink, M. Ku, K. Hochedlinger, B. E. Bernstein, and R. Jaenisch. 2007. In vitro reprogramming of fibroblasts into a pluripotent ES-cell-like state. Nature 448:318-324.

435. Shi, Y., C. Desponts, J. T. Do, H. S. Hahm, H. R. Scholer, and S. Ding. 2008. Induction of pluripotent stem cells from mouse embryonic fibroblasts by Oct 4 and Klf4 with smallmolecule compounds. Cell Stem Cell 3:568-574.

436. Zaharevitz, D. W., R. Gussio, M. Leost, A. M. Senderowicz, T. Lahusen, C. Kunick, L. Meijer, and E. A. Sausville. 1999. Discovery and initial characterization of the paullones, a novel class of small-molecule inhibitors of cyclin-dependent kinases. Cancer Res 59:2566-2569.

437. Lyssiotis, C. A., R. K. Foreman, J. Staerk, M. Garcia, D. Mathur, S. Markoulaki, J. Hanna, L. L. Lairson, B. D. Charette, L. C. Bouchez, M. Bollong, C. Kunick, A. Brinker, C. Y. Cho, P. G. Schultz, and R. Jaenisch. 2009. Reprogramming of murine fibroblasts to induced pluripotent stem cells with chemical complementation of Klf4. Proc Natl Acad Sci U S A 106:8912-8917.

438. Boyer, L. A., T. I. Lee, M. F. Cole, S. E. Johnstone, S. S. Levine, J. P. Zucker, M. G. Guenther, R. M. Kumar, H. L. Murray, R. G. Jenner, D. K. Gifford, D. A. Melton, R. Jaenisch, and R. A. Young. 2005. Core transcriptional regulatory circuitry in human embryonic stem cells. Cell 122:947-956.

439. Loh, Y. H., Q. Wu, J. L. Chew, V. B. Vega, W. Zhang, X. Chen, G. Bourque, J. George, B. Leong, J. Liu, K. Y. Wong, K. W. Sung, C. W. Lee, X. D. Zhao, K. P. Chiu, L. 
Lipovich, V. A. Kuznetsov, P. Robson, L. W. Stanton, C. L. Wei, Y. Ruan, B. Lim, and H. H. Ng. 2006. The Oct4 and Nanog transcription network regulates pluripotency in mouse embryonic stem cells. Nat Genet 38:431-440.

440. Rowland, B. D., R. Bernards, and D. S. Peeper. 2005. The KLF4 tumour suppressor is a transcriptional repressor of $\mathrm{p} 53$ that acts as a context-dependent oncogene. Nat Cell Biol 7:1074-1082.

441. Lin, T., C. Chao, S. Saito, S. J. Mazur, M. E. Murphy, E. Appella, and Y. Xu. 2005. p53 induces differentiation of mouse embryonic stem cells by suppressing Nanog expression. Nat Cell Biol 7:165-171.

442. Seoane, J., H. V. Le, and J. Massague. 2002. Myc suppression of the p21(Cip1) Cdk inhibitor influences the outcome of the p53 response to DNA damage. Nature 419:729734.

443. Zindy, F., C. M. Eischen, D. H. Randle, T. Kamijo, J. L. Cleveland, C. J. Sherr, and M. F. Roussel. 1998. Myc signaling via the ARF tumor suppressor regulates p53-dependent apoptosis and immortalization. Genes Dev 12:2424-2433.

444. Kim, J., J. Chu, X. Shen, J. Wang, and S. H. Orkin. 2008. An extended transcriptional network for pluripotency of embryonic stem cells. Cell 132:1049-1061.

445. Li, Y., J. McClintick, L. Zhong, H. J. Edenberg, M. C. Yoder, and R. J. Chan. 2005. Murine embryonic stem cell differentiation is promoted by SOCS-3 and inhibited by the zinc finger transcription factor Klf4. Blood 105:635-637.

446. Hall, J., G. Guo, J. Wray, I. Eyres, J. Nichols, L. Grotewold, S. Morfopoulou, P. Humphreys, W. Mansfield, R. Walker, S. Tomlinson, and A. Smith. 2009. Oct4 and LIF/Stat 3 additively induce Kruppel factors to sustain embryonic stem cell self-renewal. Cell Stem Cell 5:597-609.

447. Niwa, H., K. Ogawa, D. Shimosato, and K. Adachi. 2009. A parallel circuit of LIF signalling pathways maintains pluripotency of mouse ES cells. Nature 460:118-122.

448. Bourillot, P. Y., I. Aksoy, V. Schreiber, F. Wianny, H. Schulz, O. Hummel, N. Hubner, and P. Savatier. 2009. Novel STAT3 target genes exert distinct roles in the inhibition of mesoderm and endoderm differentiation in cooperation with Nanog. Stem Cells 27:17601771. 
449. Guo, G., J. Yang, J. Nichols, J. S. Hall, I. Eyres, W. Mansfield, and A. Smith. 2009. Klf4 reverts developmentally programmed restriction of ground state pluripotency. Development 136:1063-1069.

450. Hirai, H., P. Karian, and N. Kikyo. 2011. Regulation of embryonic stem cell self-renewal and pluripotency by leukaemia inhibitory factor. Biochem J 438:11-23.

451. Yoon, H. S. and V. W. Yang. 2004. Requirement of Kruppel-like factor 4 in preventing entry into mitosis following DNA damage. J Biol Chem 279:5035-5041.

452. Cho, S. D., S. Chintharlapalli, M. Abdelrahim, S. Papineni, S. Liu, J. Guo, P. Lei, A. Abudayyeh, and S. Safe. 2008. 5,5'-Dibromo-bis(3'-indolyl)methane induces Kruppellike factor 4 and p21 in colon cancer cells. Mol Cancer Ther 7:2109-2120.

453. Chen, Z. Y., X. Wang, Y. Zhou, G. Offner, and C. C. Tseng. 2005. Destabilization of Kruppel-like factor 4 protein in response to serum stimulation involves the ubiquitinproteasome pathway. Cancer Res 65:10394-10400.

454. Dang, D. T., X. Chen, J. Feng, M. Torbenson, L. H. Dang, and V. W. Yang. 2003. Overexpression of Kruppel-like factor 4 in the human colon cancer cell line RKO leads to reduced tumorigenecity. Oncogene 22:3424-3430.

455. Talmasov, D., Z. Xinjun, B. Yu, M. O. Nandan, A. B. Bialkowska, E. Elkarim, J. Kuruvilla, V. W. Yang, and A. M. Ghaleb. 2015. Kruppel-like factor 4 is a radioprotective factor for the intestine following gamma-radiation-induced gut injury in mice. Am J Physiol Gastrointest Liver Physiol 308:G121-G138.

456. Zhao, W., I. M. Hisamuddin, M. O. Nandan, B. A. Babbin, N. E. Lamb, and V. W. Yang. 2004. Identification of Kruppel-like factor 4 as a potential tumor suppressor gene in colorectal cancer. Oncogene 23:395-402.

457. Choi, B. J., Y. G. Cho, J. W. Song, C. J. Kim, S. Y. Kim, S. W. Nam, N. J. Yoo, J. Y. Lee, and W. S. Park. 2006. Altered expression of the KLF4 in colorectal cancers. Pathol Res Pract 202:585-589.

458. Patel, N. V., A. M. Ghaleb, M. O. Nandan, and V. W. Yang. 2010. Expression of the tumor suppressor Kruppel-like factor 4 as a prognostic predictor for colon cancer. Cancer Epidemiol Biomarkers Prev 19:2631-2638. 
459. Lee, H. Y., J. B. Ahn, S. Y. Rha, H. C. Chung, K. H. Park, T. S. Kim, N. K. Kim, and S. J. Shin. 2014. High KLF4 level in normal tissue predicts poor survival in colorectal cancer patients. World J Surg Oncol 12:232.

460. Hu, R., Y. Zuo, L. Zuo, C. Liu, S. Zhang, Q. Wu, Q. Zhou, S. Gui, W. Wei, and Y. Wang. 2011. KLF4 Expression Correlates with the Degree of Differentiation in Colorectal Cancer. Gut Liver 5:154-159.

461. Dang, D. T., K. E. Bachman, C. S. Mahatan, L. H. Dang, F. M. Giardiello, and V. W. Yang. 2000. Decreased expression of the gut-enriched Kruppel-like factor gene in intestinal adenomas of multiple intestinal neoplasia mice and in colonic adenomas of familial adenomatous polyposis patients. FEBS Lett 476:203-207.

462. Kimelman, D. and W. Xu. 2006. beta-catenin destruction complex: insights and questions from a structural perspective. Oncogene 25:7482-7491.

463. Polakis, P. 2000. Wnt signaling and cancer. Genes Dev 14:1837-1851.

464. Stone, C. D., Z. Y. Chen, and C. C. Tseng. 2002. Gut-enriched Kruppel-like factor regulates colonic cell growth through APC/beta-catenin pathway. FEBS Lett 530:147152.

465. Ma, M. Q., H. D. Zhang, P. Tang, H. J. Jiang, and C. G. Chen. 2014. Association of Kruppel-like factor 4 expression with the prognosis of esophageal squamous cell carcinoma patients. Int J Clin Exp Pathol 7:6679-6685.

466. Wei, D., W. Gong, M. Kanai, C. Schlunk, L. Wang, J. C. Yao, T. T. Wu, S. Huang, and K. Xie. 2005. Drastic down-regulation of Kruppel-like factor 4 expression is critical in human gastric cancer development and progression. Cancer Res 65:2746-2754.

467. Zhang, N., J. Zhang, Z. W. Wang, L. Zha, and Z. Huang. 2012. Altered expression of Kruppel-like factor 4 and beta-catenin in human gastric cancer. Oncol Lett 3:1017-1022.

468. Lin, Z. S., H. C. Chu, Y. C. Yen, B. C. Lewis, and Y. W. Chen. 2012. Kruppel-like factor 4, a tumor suppressor in hepatocellular carcinoma cells reverts epithelial mesenchymal transition by suppressing slug expression. PLoS One 7:e43593.

469. Hsu, H. T., P. R. Wu, C. J. Chen, L. S. Hsu, C. M. Yeh, M. T. Hsing, Y. S. Chiang, M. T. Lai, and K. T. Yeh. 2014. High cytoplasmic expression of Kruppel-like factor 4 is an 
independent prognostic factor of better survival in hepatocellular carcinoma. Int J Mol Sci 15:9894-9906.

470. Abrigo, M., R. Alvarez, M. L. Paparella, D. E. Calb, J. E. Bal de Kier, J. S. Gutkind, and A. R. Raimondi. 2014. Impairing squamous differentiation by Klf4 deletion is sufficient to initiate tongue carcinoma development upon K-Ras activation in mice. Carcinogenesis 35:662-669.

471. Wei, D., M. Kanai, Z. Jia, X. Le, and K. Xie. 2008. Kruppel-like factor 4 induces p27Kip1 expression in and suppresses the growth and metastasis of human pancreatic cancer cells. Cancer Res 68:4631-4639.

472. Zammarchi, F., M. Morelli, M. Menicagli, C. C. Di, K. Zavaglia, A. Paolucci, D. Campani, P. Aretini, U. Boggi, F. Mosca, A. Cavazzana, L. Cartegni, G. Bevilacqua, and C. M. Mazzanti. 2011. KLF4 is a novel candidate tumor suppressor gene in pancreatic ductal carcinoma. Am J Pathol 178:361-372.

473. El-Karim, E. A., E. G. Hagos, A. M. Ghaleb, B. Yu, and V. W. Yang. 2013. Kruppel-like factor 4 regulates genetic stability in mouse embryonic fibroblasts. Mol Cancer 12:89.

474. Hagos, E. G., A. M. Ghaleb, W. B. Dalton, A. B. Bialkowska, and V. W. Yang. 2009. Mouse embryonic fibroblasts null for the Kruppel-like factor 4 gene are genetically unstable. Oncogene 28:1197-1205.

475. Chen, Z., Y. Wang, W. Liu, G. Zhao, S. Lee, A. Balogh, Y. Zou, Y. Guo, Z. Zhang, W. Gu, C. Li, G. Tigyi, and J. Yue. 2014. Doxycycline inducible Kruppel-like factor 4 lentiviral vector mediates mesenchymal to epithelial transition in ovarian cancer cells. PLoS One 9:e105331.

476. Foster, K. W., A. R. Frost, P. Kie-Bell, C. Y. Lin, J. A. Engler, W. E. Grizzle, and J. M. Ruppert. 2000. Increase of GKLF messenger RNA and protein expression during progression of breast cancer. Cancer Res 60:6488-6495.

477. Pandya, A. Y., L. I. Talley, A. R. Frost, T. J. Fitzgerald, V. Trivedi, M. Chakravarthy, D. C. Chhieng, W. E. Grizzle, J. A. Engler, H. Krontiras, K. I. Bland, A. F. LoBuglio, S. M. Lobo-Ruppert, and J. M. Ruppert. 2004. Nuclear localization of KLF4 is associated with an aggressive phenotype in early-stage breast cancer. Clin Cancer Res 10:2709-2719.

478. Cancer Genome Atlas Network. 2012. Comprehensive molecular portraits of human breast tumours. Nature 490:61-70. 
479. Farrugia, M. K., S. B. Sharma, C. C. Lin, S. L. McLaughlin, D. B. Vanderbilt, A. G. Ammer, M. A. Salkeni, P. Stoilov, Y. M. Agazie, C. J. Creighton, and J. M. Ruppert. 2015. Regulation of anti-apoptotic signaling by Kruppel-like factors 4 and 5 mediates lapatinib resistance in breast cancer. Cell Death Dis 6:e1699.

480. Herschkowitz, J. I., K. Simin, V. J. Weigman, I. Mikaelian, J. Usary, Z. Hu, K. E. Rasmussen, L. P. Jones, S. Assefnia, S. Chandrasekharan, M. G. Backlund, Y. Yin, A. I. Khramtsov, R. Bastein, J. Quackenbush, R. I. Glazer, P. H. Brown, J. E. Green, L. Kopelovich, P. A. Furth, J. P. Palazzo, O. I. Olopade, P. S. Bernard, G. A. Churchill, D. T. Van, and C. M. Perou. 2007. Identification of conserved gene expression features between murine mammary carcinoma models and human breast tumors. Genome Biol $8:$ R76.

481. Yori, J. L., D. D. Seachrist, E. Johnson, K. L. Lozada, F. W. bdul-Karim, L. A. Chodosh, W. P. Schiemann, and R. A. Keri. 2011. Kruppel-like factor 4 inhibits tumorigenic progression and metastasis in a mouse model of breast cancer. Neoplasia 13:601-610.

482. Akaogi, K., Y. Nakajima, I. Ito, S. Kawasaki, S. H. Oie, A. Murayama, K. Kimura, and J. Yanagisawa. 2009. KLF4 suppresses estrogen-dependent breast cancer growth by inhibiting the transcriptional activity of ERalpha. Oncogene 28:2894-2902.

483. Okuda, H., F. Xing, P. R. Pandey, S. Sharma, M. Watabe, S. K. Pai, Y. Y. Mo, M. Iiizumi-Gairani, S. Hirota, Y. Liu, K. Wu, R. Pochampally, and K. Watabe. 2013. miR-7 suppresses brain metastasis of breast cancer stem-like cells by modulating KLF4. Cancer Res 73:1434-1444.

484. Lin, C. C., S. B. Sharma, M. K. Farrugia, S. L. McLaughlin, R. J. Ice, Y. V. Loskutov, E. N. Pugacheva, K. M. Brundage, D. Chen, and J. M. Ruppert. 2015. Kruppel-like factor 4 signals through microRNA-206 to promote tumor initiation and cell survival. Oncogenesis 4:e155.

485. Sharma, S. B., C. C. Lin, M. K. Farrugia, S. L. McLaughlin, E. J. Ellis, K. M. Brundage, M. A. Salkeni, and J. M. Ruppert. 2014. MicroRNAs 206 and 21 cooperate to promote RAS-extracellular signal-regulated kinase signaling by suppressing the translation of RASA1 and SPRED1. Mol Cell Biol 34:4143-4164.

486. Duncan, J. S., M. C. Whittle, K. Nakamura, A. N. Abell, A. A. Midland, J. S. Zawistowski, N. L. Johnson, D. A. Granger, N. V. Jordan, D. B. Darr, J. Usary, P. F. Kuan, D. M. Smalley, B. Major, X. He, K. A. Hoadley, B. Zhou, N. E. Sharpless, C. M. Perou, W. Y. Kim, S. M. Gomez, X. Chen, J. Jin, S. V. Frye, H. S. Earp, L. M. Graves, 
and G. L. Johnson. 2012. Dynamic reprogramming of the kinome in response to targeted MEK inhibition in triple-negative breast cancer. Cell 149:307-321.

487. Dontu, G., W. M. Abdallah, J. M. Foley, K. W. Jackson, M. F. Clarke, M. J. Kawamura, and M. S. Wicha. 2003. In vitro propagation and transcriptional profiling of human mammary stem/progenitor cells. Genes Dev 17:1253-1270.

488. Yu, F., J. Li, H. Chen, J. Fu, S. Ray, S. Huang, H. Zheng, and W. Ai. 2011. Kruppel-like factor 4 (KLF4) is required for maintenance of breast cancer stem cells and for cell migration and invasion. Oncogene 30:2161-2172.

489. Illa-Bochaca, I., R. Fernandez-Gonzalez, D. N. Shelton, B. E. Welm, C. Ortiz-deSolorzano, and M. H. Barcellos-Hoff. 2010. Limiting-dilution transplantation assays in mammary stem cell studies. Methods Mol Biol 621:29-47.

490. Burk, U., J. Schubert, U. Wellner, O. Schmalhofer, E. Vincan, S. Spaderna, and T. Brabletz. 2008. A reciprocal repression between ZEB1 and members of the miR-200 family promotes EMT and invasion in cancer cells. EMBO Rep 9:582-589.

491. Bracken, C. P., P. A. Gregory, N. Kolesnikoff, A. G. Bert, J. Wang, M. F. Shannon, and G. J. Goodall. 2008. A double-negative feedback loop between ZEB1-SIP1 and the microRNA-200 family regulates epithelial-mesenchymal transition. Cancer Res 68:78467854.

492. Christoffersen, N. R., A. Silahtaroglu, U. A. Orom, S. Kauppinen, and A. H. Lund. 2007. miR-200b mediates post-transcriptional repression of ZFHX1B. RNA. 13:1172-1178.

493. Hurteau, G. J., J. A. Carlson, S. D. Spivack, and G. J. Brock. 2007. Overexpression of the microRNA hsa-miR-200c leads to reduced expression of transcription factor 8 and increased expression of E-cadherin. Cancer Res 67:7972-7976.

494. Gregory, P. A., A. G. Bert, E. L. Paterson, S. C. Barry, A. Tsykin, G. Farshid, M. A. Vadas, Y. Khew-Goodall, and G. J. Goodall. 2008. The miR-200 family and miR-205 regulate epithelial to mesenchymal transition by targeting ZEB1 and SIP1. Nat Cell Biol 10:593-601.

495. Korpal, M., E. S. Lee, G. Hu, and Y. Kang. 2008. The miR-200 family inhibits epithelialmesenchymal transition and cancer cell migration by direct targeting of E-cadherin transcriptional repressors ZEB1 and ZEB2. J Biol Chem 283:14910-14914. 
496. Park, S. M., A. B. Gaur, E. Lengyel, and M. E. Peter. 2008. The miR-200 family determines the epithelial phenotype of cancer cells by targeting the E-cadherin repressors ZEB1 and ZEB2. Genes Dev 22:894-907.

497. Shimono, Y., M. Zabala, R. W. Cho, N. Lobo, P. Dalerba, D. Qian, M. Diehn, H. Liu, S. P. Panula, E. Chiao, F. M. Dirbas, G. Somlo, R. A. Pera, K. Lao, and M. F. Clarke. 2009. Downregulation of miRNA-200c links breast cancer stem cells with normal stem cells. Cell 138:592-603.

498. Hanahan, D. and R. A. Weinberg. 2000. The hallmarks of cancer. Cell 100:57-70.

499. Hanahan, D. and R. A. Weinberg. 2011. Hallmarks of cancer: the next generation. Cell 144:646-674.

500. Siegel, R., D. Naishadham, and A. Jemal. 2013. Cancer statistics, 2013. CA Cancer J Clin 63:11-30.

501. Newman, L. A. 2009. Epidemiology of locally advanced breast cancer. Semin. Radiat Oncol 19:195-203.

502. Briasoulis, E., V. Karavasilis, L. Kostadima, M. Ignatiadis, G. Fountzilas, and N. Pavlidis. 2004. Metastatic breast carcinoma confined to bone: portrait of a clinical entity. Cancer 101:1524-1528.

503. Sherry, M. M., F. A. Greco, D. H. Johnson, and J. D. Hainsworth. 1986. Metastatic breast cancer confined to the skeletal system. An indolent disease. Am J Med 81:381-386.

504. Coleman, R. E., P. Smith, and R. D. Rubens. 1998. Clinical course and prognostic factors following bone recurrence from breast cancer. Br. J Cancer 77:336-340.

505. Lee, Y. T. 1983. Breast carcinoma: pattern of metastasis at autopsy. J Surg Oncol 23:175180.

506. Chua, T. C., A. Saxena, W. Liauw, F. Chu, and D. L. Morris. 2011. Hepatic resection for metastatic breast cancer: a systematic review. Eur J Cancer 47:2282-2290.

507. Friedel, G., U. Pastorino, R. J. Ginsberg, P. Goldstraw, M. Johnston, H. Pass, J. B. Putnam, H. Toomes, and L. E. International Registry of Lung Metastases. 2002. Results 
of lung metastasectomy from breast cancer: prognostic criteria on the basis of 467 cases of the International Registry of Lung Metastases. Eur J Cardiothorac. Surg 22:335-344.

508. McDonald, M. L., C. Deschamps, D. M. Ilstrup, M. S. Allen, V. F. Trastek, and P. C. Pairolero. 1994. Pulmonary resection for metastatic breast cancer. Ann Thorac Surg 58:1599-1602.

509. Yoshimoto, M., K. Tada, S. Nishimura, M. Makita, T. Iwase, F. Kasumi, S. Okumura, Y. Sato, and K. Nakagawa. 2008. Favourable long-term results after surgical removal of lung metastases of breast cancer. Breast Cancer Res Treat 110:485-491.

510. Meimarakis, G., D. Ruttinger, J. Stemmler, A. Crispin, R. Weidenhagen, M. Angele, J. Fertmann, R. A. Hatz, and H. Winter. 2013. Prolonged overall survival after pulmonary metastasectomy in patients with breast cancer. Ann Thorac Surg 95:1170-1180.

511. Planchard, D., J. C. Soria, S. Michiels, D. Grunenwald, P. Validire, R. Caliandro, P. Girard, and C. T. Le. 2004. Uncertain benefit from surgery in patients with lung metastases from breast carcinoma. Cancer 100:28-35.

512. Barnholtz-Sloan, J. S., A. E. Sloan, F. G. Davis, F. D. Vigneau, P. Lai, and R. E. Sawaya. 2004. Incidence proportions of brain metastases in patients diagnosed (1973 to 2001) in the Metropolitan Detroit Cancer Surveillance System. J Clin Oncol 22:2865-2872.

513. Schouten, L. J., J. Rutten, H. A. Huveneers, and A. Twijnstra. 2002. Incidence of brain metastases in a cohort of patients with carcinoma of the breast, colon, kidney, and lung and melanoma. Cancer 94:2698-2705.

514. Tsukada, Y., A. Fouad, J. W. Pickren, and W. W. Lane. 1983. Central nervous system metastasis from breast carcinoma. Autopsy study. Cancer 52:2349-2354.

515. Lin, N. U., E. Claus, J. Sohl, A. R. Razzak, A. Arnaout, and E. P. Winer. 2008. Sites of distant recurrence and clinical outcomes in patients with metastatic triple-negative breast cancer: high incidence of central nervous system metastases. Cancer 113:2638-2645.

516. Arvold, N. D., K. S. Oh, A. Niemierko, A. G. Taghian, N. U. Lin, R. F. bi-Raad, M. Sreedhara, J. R. Harris, and B. M. Alexander. 2012. Brain metastases after breastconserving therapy and systemic therapy: incidence and characteristics by biologic subtype. Breast Cancer Res Treat 136:153-160. 
517. Chia, S. K., C. H. Speers, Y. D'yachkova, A. Kang, S. Malfair-Taylor, J. Barnett, A. Coldman, K. A. Gelmon, S. E. O'reilly, and I. A. Olivotto. 2007. The impact of new chemotherapeutic and hormone agents on survival in a population-based cohort of women with metastatic breast cancer. Cancer 110:973-979.

518. Gennari, A., P. Conte, R. Rosso, C. Orlandini, and P. Bruzzi. 2005. Survival of metastatic breast carcinoma patients over a 20-year period: a retrospective analysis based on individual patient data from six consecutive studies. Cancer 104:1742-1750.

519. Dafni, U., I. Grimani, A. Xyrafas, A. G. Eleftheraki, and G. Fountzilas. 2010. Fifteenyear trends in metastatic breast cancer survival in Greece. Breast Cancer Res Treat 119:621-631.

520. Greenberg, P. A., G. N. Hortobagyi, T. L. Smith, L. D. Ziegler, D. K. Frye, and A. U. Buzdar. 1996. Long-term follow-up of patients with complete remission following combination chemotherapy for metastatic breast cancer. J Clin Oncol 14:2197-2205.

521. Kiely, B. E., Y. Y. Soon, M. H. Tattersall, and M. R. Stockler. 2011. How long have I got? Estimating typical, best-case, and worst-case scenarios for patients starting first-line chemotherapy for metastatic breast cancer: a systematic review of recent randomized trials. J Clin Oncol 29:456-463.

522. Thientosapol, E. S., T. T. Tran, S. A. la-Fiorentina, D. H. Adams, L. Chantrill, M. R. Stockler, and B. E. Kiely. 2013. Survival times of women with metastatic breast cancer starting first-line chemotherapy in routine clinical practice versus contemporary randomised trials. Intern Med J 43:883-888.

523. Parise, C. A., K. R. Bauer, M. M. Brown, and V. Caggiano. 2009. Breast cancer subtypes as defined by the estrogen receptor (ER), progesterone receptor (PR), and the human epidermal growth factor receptor 2 (HER2) among women with invasive breast cancer in California, 1999-2004. Breast J 15:593-602.

524. Prat, A., A. Lluch, J. Albanell, W. T. Barry, C. Fan, J. I. Chacon, J. S. Parker, L. Calvo, A. Plazaola, A. Arcusa, M. A. Segui-Palmer, O. Burgues, N. Ribelles, A. RodriguezLescure, A. Guerrero, M. Ruiz-Borrego, B. Munarriz, J. A. Lopez, B. Adamo, M. C. Cheang, Y. Li, Z. Hu, M. L. Gulley, M. J. Vidal, B. N. Pitcher, M. C. Liu, M. L. Citron, M. J. Ellis, E. Mardis, T. Vickery, C. A. Hudis, E. P. Winer, L. A. Carey, R. Caballero, E. Carrasco, M. Martin, C. M. Perou, and E. Alba. 2014. Predicting response and survival in chemotherapy-treated triple-negative breast cancer. Br. J Cancer 111:1532-1541. 
525. O'Brien, K. M., S. R. Cole, C. K. Tse, C. M. Perou, L. A. Carey, W. D. Foulkes, L. G. Dressler, J. Geradts, and R. C. Millikan. 2010. Intrinsic breast tumor subtypes, race, and long-term survival in the Carolina Breast Cancer Study. Clin Cancer Res 16:6100-6110.

526. Perou, C. M., T. Sorlie, M. B. Eisen, R. M. van de, S. S. Jeffrey, C. A. Rees, J. R. Pollack, D. T. Ross, H. Johnsen, L. A. Akslen, O. Fluge, A. Pergamenschikov, C. Williams, S. X. Zhu, P. E. Lonning, A. L. Borresen-Dale, P. O. Brown, and D. Botstein. 2000. Molecular portraits of human breast tumours. Nature 406:747-752.

527. Nielsen, T. O., F. D. Hsu, K. Jensen, M. Cheang, G. Karaca, Z. Hu, T. HernandezBoussard, C. Livasy, D. Cowan, L. Dressler, L. A. Akslen, J. Ragaz, A. M. Gown, C. B. Gilks, R. M. van de, and C. M. Perou. 2004. Immunohistochemical and clinical characterization of the basal-like subtype of invasive breast carcinoma. Clin Cancer Res 10:5367-5374.

528. Carey, L. A., C. M. Perou, C. A. Livasy, L. G. Dressler, D. Cowan, K. Conway, G. Karaca, M. A. Troester, C. K. Tse, S. Edmiston, S. L. Deming, J. Geradts, M. C. Cheang, T. O. Nielsen, P. G. Moorman, H. S. Earp, and R. C. Millikan. 2006. Race, breast cancer subtypes, and survival in the Carolina Breast Cancer Study. JAMA 295:2492-2502.

529. Fan, C., D. S. Oh, L. Wessels, B. Weigelt, D. S. Nuyten, A. B. Nobel, L. J. van't Veer, and C. M. Perou. 2006. Concordance among gene-expression-based predictors for breast cancer. N Engl J Med 355:560-569.

530. Carey, L. A., E. C. Dees, L. Sawyer, L. Gatti, D. T. Moore, F. Collichio, D. W. Ollila, C. I. Sartor, M. L. Graham, and C. M. Perou. 2007. The triple negative paradox: primary tumor chemosensitivity of breast cancer subtypes. Clin Cancer Res 13:2329-2334.

531. Gunzel, D. and A. S. Yu. 2013. Claudins and the modulation of tight junction permeability. Physiol Rev 93:525-569.

532. Hennessy, B. T., A. M. Gonzalez-Angulo, K. Stemke-Hale, M. Z. Gilcrease, S. Krishnamurthy, J. S. Lee, J. Fridlyand, A. Sahin, R. Agarwal, C. Joy, W. Liu, D. Stivers, K. Baggerly, M. Carey, A. Lluch, C. Monteagudo, X. He, V. Weigman, C. Fan, J. Palazzo, G. N. Hortobagyi, L. K. Nolden, N. J. Wang, V. Valero, J. W. Gray, C. M. Perou, and G. B. Mills. 2009. Characterization of a naturally occurring breast cancer subset enriched in epithelial-to-mesenchymal transition and stem cell characteristics. Cancer Res 69:4116-4124.

533. Creighton, C. J., X. Li, M. Landis, J. M. Dixon, V. M. Neumeister, A. Sjolund, D. L. Rimm, H. Wong, A. Rodriguez, J. I. Herschkowitz, C. Fan, X. Zhang, X. He, A. Pavlick, 
M. C. Gutierrez, L. Renshaw, A. A. Larionov, D. Faratian, S. G. Hilsenbeck, C. M. Perou, M. T. Lewis, J. M. Rosen, and J. C. Chang. 2009. Residual breast cancers after conventional therapy display mesenchymal as well as tumor-initiating features. Proc Natl Acad Sci U S A 106:13820-13825.

534. Prat, A., J. S. Parker, O. Karginova, C. Fan, C. Livasy, J. I. Herschkowitz, X. He, and C. M. Perou. 2010. Phenotypic and molecular characterization of the claudin-low intrinsic subtype of breast cancer. Breast Cancer Res 12:R68.

535. Prat, A. and C. M. Perou. 2011. Deconstructing the molecular portraits of breast cancer. Mol Oncol 5:5-23.

536. Prat, A., O. Karginova, J. S. Parker, C. Fan, X. He, L. Bixby, J. C. Harrell, E. Roman, B. Adamo, M. Troester, and C. M. Perou. 2013. Characterization of cell lines derived from breast cancers and normal mammary tissues for the study of the intrinsic molecular subtypes. Breast Cancer Res Treat 142:237-255.

537. Iorns, E., K. Drews-Elger, T. M. Ward, S. Dean, J. Clarke, D. Berry, A. D. El, and M. Lippman. 2012. A new mouse model for the study of human breast cancer metastasis. PLoS One 7:e47995.

538. Lee, J. H. and D. R. Welch. 1997. Suppression of metastasis in human breast carcinoma MDA-MB-435 cells after transfection with the metastasis suppressor gene, KiSS-1. Cancer Res 57:2384-2387.

539. Maroulakou, I. G., M. Anver, L. Garrett, and J. E. Green. 1994. Prostate and mammary adenocarcinoma in transgenic mice carrying a rat C3(1) simian virus 40 large tumor antigen fusion gene. Proc Natl Acad Sci U S A 91:11236-11240.

540. Green, J. E., M. A. Shibata, K. Yoshidome, M. L. Liu, C. Jorcyk, M. R. Anver, J. Wigginton, R. Wiltrout, E. Shibata, S. Kaczmarczyk, W. Wang, Z. Y. Liu, A. Calvo, and C. Couldrey. 2000. The C3(1)/SV40 T-antigen transgenic mouse model of mammary cancer: ductal epithelial cell targeting with multistage progression to carcinoma. Oncogene 19:1020-1027.

541. Holzer, R. G., C. MacDougall, G. Cortright, K. Atwood, J. E. Green, and C. L. Jorcyk. 2003. Development and characterization of a progressive series of mammary adenocarcinoma cell lines derived from the C3(1)/SV40 Large T-antigen transgenic mouse model. Breast Cancer Res Treat 77:65-76. 
542. Fidler, I. J. 2003. The pathogenesis of cancer metastasis: the 'seed and soil' hypothesis revisited. Nat Rev Cancer 3:453-458.

543. Valastyan, S. and R. A. Weinberg. 2011. Tumor metastasis: molecular insights and evolving paradigms. Cell 147:275-292.

544. Saxena, M. and G. Christofori. 2013. Rebuilding cancer metastasis in the mouse. Mol Oncol 7:283-296.

545. Vanharanta, S. and J. Massague. 2013. Origins of metastatic traits. Cancer Cell 24:410421.

546. Eccles, S. A. and D. R. Welch. 2007. Metastasis: recent discoveries and novel treatment strategies. Lancet 369:1742-1757.

547. Chambers, A. F., A. C. Groom, and I. C. MacDonald. 2002. Dissemination and growth of cancer cells in metastatic sites. Nat Rev Cancer 2:563-572.

548. Nguyen, D. X., P. D. Bos, and J. Massague. 2009. Metastasis: from dissemination to organ-specific colonization. Nat Rev Cancer 9:274-284.

549. 2011. AJCC Cancer Staging Manual. Springer.

550. Wittekind, C. and M. Neid. 2005. Cancer invasion and metastasis. Oncology 69 Suppl 1:14-16.

551. Tait, C. R., D. Dodwell, and K. Horgan. 2004. Do metastases metastasize? J Pathol 203:515-518.

552. Kim, M. Y., T. Oskarsson, S. Acharyya, D. X. Nguyen, X. H. Zhang, L. Norton, and J. Massague. 2009. Tumor self-seeding by circulating cancer cells. Cell 139:1315-1326.

553. Comen, E., L. Norton, and J. Massague. 2011. Clinical implications of cancer selfseeding. Nat Rev Clin Oncol 8:369-377.

554. Lucci, A., C. S. Hall, A. K. Lodhi, A. Bhattacharyya, A. E. Anderson, L. Xiao, I. Bedrosian, H. M. Kuerer, and S. Krishnamurthy. 2012. Circulating tumour cells in nonmetastatic breast cancer: a prospective study. Lancet Oncol 13:688-695. 
555. Rack, B., C. Schindlbeck, J. Juckstock, U. Andergassen, P. Hepp, T. Zwingers, T. W. Friedl, R. Lorenz, H. Tesch, P. A. Fasching, T. Fehm, A. Schneeweiss, W. Lichtenegger, M. W. Beckmann, K. Friese, K. Pantel, W. Janni, and SUCCESS Study Group. 2014. Circulating tumor cells predict survival in early average-to-high risk breast cancer patients. J Natl Cancer Inst 106.

556. Xenidis, N., M. Perraki, M. Kafousi, S. Apostolaki, I. Bolonaki, A. Stathopoulou, K. Kalbakis, N. Androulakis, C. Kouroussis, T. Pallis, C. Christophylakis, K. Argyraki, E. S. Lianidou, S. Stathopoulos, V. Georgoulias, and D. Mavroudis. 2006. Predictive and prognostic value of peripheral blood cytokeratin-19 mRNA-positive cells detected by real-time polymerase chain reaction in node-negative breast cancer patients. J Clin Oncol 24:3756-3762.

557. Cristofanilli, M., D. F. Hayes, G. T. Budd, M. J. Ellis, A. Stopeck, J. M. Reuben, G. V. Doyle, J. Matera, W. J. Allard, M. C. Miller, H. A. Fritsche, G. N. Hortobagyi, and L. W. Terstappen. 2005. Circulating tumor cells: a novel prognostic factor for newly diagnosed metastatic breast cancer. J Clin Oncol 23:1420-1430.

558. Hayes, D. F., M. Cristofanilli, G. T. Budd, M. J. Ellis, A. Stopeck, M. C. Miller, J. Matera, W. J. Allard, G. V. Doyle, and L. W. Terstappen. 2006. Circulating tumor cells at each follow-up time point during therapy of metastatic breast cancer patients predict progression-free and overall survival. Clin Cancer Res 12:4218-4224.

559. Cristofanilli, M., K. R. Broglio, V. Guarneri, S. Jackson, H. A. Fritsche, R. Islam, S. Dawood, J. M. Reuben, S. W. Kau, J. M. Lara, S. Krishnamurthy, N. T. Ueno, G. N. Hortobagyi, and V. Valero. 2007. Circulating tumor cells in metastatic breast cancer: biologic staging beyond tumor burden. Clin Breast Cancer 7:471-479.

560. Dawood, S., K. Broglio, V. Valero, J. Reuben, B. Handy, R. Islam, S. Jackson, G. N. Hortobagyi, H. Fritsche, and M. Cristofanilli. 2008. Circulating tumor cells in metastatic breast cancer: from prognostic stratification to modification of the staging system? Cancer 113:2422-2430.

561. Smerage, J. B., W. E. Barlow, G. N. Hortobagyi, E. P. Winer, B. Leyland-Jones, G. Srkalovic, S. Tejwani, A. F. Schott, M. A. O'Rourke, D. L. Lew, G. V. Doyle, J. R. Gralow, R. B. Livingston, and D. F. Hayes. 2014. Circulating tumor cells and response to chemotherapy in metastatic breast cancer: SWOG S0500. J Clin Oncol 32:3483-3489.

562. Magbanua, M. J., L. A. Carey, A. DeLuca, J. Hwang, J. H. Scott, M. F. Rimawi, E. L. Mayer, P. K. Marcom, M. C. Liu, F. J. Esteva, J. W. Park, H. S. Rugo, and Translational 
Breast Cancer Research Consortium. 2015. Circulating tumor cell analysis in metastatic triple-negative breast cancers. Clin Cancer Res 21:1098-1105.

563. Zhang, L., S. Riethdorf, G. Wu, T. Wang, K. Yang, G. Peng, J. Liu, and K. Pantel. 2012. Meta-analysis of the prognostic value of circulating tumor cells in breast cancer. Clin Cancer Res 18:5701-5710.

564. Budd, G. T., M. Cristofanilli, M. J. Ellis, A. Stopeck, E. Borden, M. C. Miller, J. Matera, M. Repollet, G. V. Doyle, L. W. Terstappen, and D. F. Hayes. 2006. Circulating tumor cells versus imaging--predicting overall survival in metastatic breast cancer. Clin Cancer Res 12:6403-6409.

565. Danila, D. C., M. Fleisher, and H. I. Scher. 2011. Circulating tumor cells as biomarkers in prostate cancer. Clin Cancer Res 17:3903-3912.

566. Mocellin, S., D. Hoon, A. Ambrosi, D. Nitti, and C. R. Rossi. 2006. The prognostic value of circulating tumor cells in patients with melanoma: a systematic review and metaanalysis. Clin Cancer Res 12:4605-4613.

567. Cohen, S. J., C. J. Punt, N. Iannotti, B. H. Saidman, K. D. Sabbath, N. Y. Gabrail, J. Picus, M. A. Morse, E. Mitchell, M. C. Miller, G. V. Doyle, H. Tissing, L. W. Terstappen, and N. J. Meropol. 2009. Prognostic significance of circulating tumor cells in patients with metastatic colorectal cancer. Ann Oncol 20:1223-1229.

568. Krebs, M. G., R. L. Metcalf, L. Carter, G. Brady, F. H. Blackhall, and C. Dive. 2014. Molecular analysis of circulating tumour cells-biology and biomarkers. Nat Rev Clin Oncol 11:129-144.

569. Alix-Panabieres, C. and K. Pantel. 2014. Challenges in circulating tumour cell research. Nat Rev Cancer 14:623-631.

570. van de Stolpe, A., K. Pantel, S. Sleijfer, L. W. Terstappen, and J. M. den Toonder. 2011. Circulating tumor cell isolation and diagnostics: toward routine clinical use. Cancer Res 71:5955-5960.

571. Powell, A. A., A. H. Talasaz, H. Zhang, M. A. Coram, A. Reddy, G. Deng, M. L. Telli, R. H. Advani, R. W. Carlson, J. A. Mollick, S. Sheth, A. W. Kurian, J. M. Ford, F. E. Stockdale, S. R. Quake, R. F. Pease, M. N. Mindrinos, G. Bhanot, S. H. Dairkee, R. W. Davis, and S. S. Jeffrey. 2012. Single cell profiling of circulating tumor cells: 
transcriptional heterogeneity and diversity from breast cancer cell lines. PLoS One 7:e33788.

572. Steeg, P. S., G. Bevilacqua, L. Kopper, U. P. Thorgeirsson, J. E. Talmadge, L. A. Liotta, and M. E. Sobel. 1988. Evidence for a novel gene associated with low tumor metastatic potential. J Natl Cancer Inst 80:200-204.

573. Leone, A., U. Flatow, K. VanHoutte, and P. S. Steeg. 1993. Transfection of human nm23-H1 into the human MDA-MB-435 breast carcinoma cell line: effects on tumor metastatic potential, colonization and enzymatic activity. Oncogene 8:2325-2333.

574. Yoshida, B. A., M. M. Sokoloff, D. R. Welch, and C. W. Rinker-Schaeffer. 2000. Metastasis-suppressor genes: a review and perspective on an emerging field. J Natl Cancer Inst 92:1717-1730.

575. Bohl, C. R., S. Harihar, W. L. Denning, R. Sharma, and D. R. Welch. 2014. Metastasis suppressors in breast cancers: mechanistic insights and clinical potential. J Mol Med (Berl) 92:13-30.

576. Welch, D. R. 1997. Technical considerations for studying cancer metastasis in vivo. Clin Exp Metastasis 15:272-306.

577. Bosma, G. C., R. P. Custer, and M. J. Bosma. 1983. A severe combined immunodeficiency mutation in the mouse. Nature 301:527-530.

578. Shultz, L. D., P. A. Schweitzer, S. W. Christianson, B. Gott, I. B. Schweitzer, B. Tennent, S. McKenna, L. Mobraaten, T. V. Rajan, D. L. Greiner, and . 1995. Multiple defects in innate and adaptive immunologic function in NOD/LtSz-scid mice. J Immunol 154:180191.

579. Kataoka, S., J. Satoh, H. Fujiya, T. Toyota, R. Suzuki, K. Itoh, and K. Kumagai. 1983. Immunologic aspects of the nonobese diabetic (NOD) mouse. Abnormalities of cellular immunity. Diabetes 32:247-253.

580. Baxter, A. G. and A. Cooke. 1993. Complement lytic activity has no role in the pathogenesis of autoimmune diabetes in NOD mice. Diabetes 42:1574-1578.

581. Shultz, L. D., B. L. Lyons, L. M. Burzenski, B. Gott, X. Chen, S. Chaleff, M. Kotb, S. D. Gillies, M. King, J. Mangada, D. L. Greiner, and R. Handgretinger. 2005. Human 
lymphoid and myeloid cell development in NOD/LtSz-scid IL2R gamma null mice engrafted with mobilized human hemopoietic stem cells. J Immunol 174:6477-6489.

582. Sugamura, K., H. Asao, M. Kondo, N. Tanaka, N. Ishii, M. Nakamura, and T. Takeshita. 1995. The common gamma-chain for multiple cytokine receptors. Adv Immunol 59:225277.

583. Russell, S. M., A. D. Keegan, N. Harada, Y. Nakamura, M. Noguchi, P. Leland, M. C. Friedmann, A. Miyajima, R. K. Puri, W. E. Paul, and . 1993. Interleukin-2 receptor gamma chain: a functional component of the interleukin-4 receptor. Science 262:18801883.

584. Noguchi, M., Y. Nakamura, S. M. Russell, S. F. Ziegler, M. Tsang, X. Cao, and W. J. Leonard. 1993. Interleukin-2 receptor gamma chain: a functional component of the interleukin-7 receptor. Science 262:1877-1880.

585. Russell, S. M., J. A. Johnston, M. Noguchi, M. Kawamura, C. M. Bacon, M. Friedmann, M. Berg, D. W. McVicar, B. A. Witthuhn, O. Silvennoinen, and . 1994. Interaction of IL2R beta and gamma c chains with Jak1 and Jak3: implications for XSCID and XCID. Science 266:1042-1045.

586. Giri, J. G., M. Ahdieh, J. Eisenman, K. Shanebeck, K. Grabstein, S. Kumaki, A. Namen, L. S. Park, D. Cosman, and D. Anderson. 1994. Utilization of the beta and gamma chains of the IL-2 receptor by the novel cytokine IL-15. EMBO J 13:2822-2830.

587. Asao, H., C. Okuyama, S. Kumaki, N. Ishii, S. Tsuchiya, D. Foster, and K. Sugamura. 2001. Cutting edge: the common gamma-chain is an indispensable subunit of the IL-21 receptor complex. J Immunol 167:1-5.

588. Sugamura, K., H. Asao, M. Kondo, N. Tanaka, N. Ishii, K. Ohbo, M. Nakamura, and T. Takeshita. 1996. The interleukin-2 receptor gamma chain: its role in the multiple cytokine receptor complexes and T cell development in XSCID. Annu Rev Immunol 14:179-205.

589. DiSanto, J. P., W. Muller, D. Guy-Grand, A. Fischer, and K. Rajewsky. 1995. Lymphoid development in mice with a targeted deletion of the interleukin 2 receptor gamma chain. Proc Natl Acad Sci U S A 92:377-381.

590. Vivier, E., E. Tomasello, M. Baratin, T. Walzer, and S. Ugolini. 2008. Functions of natural killer cells. Nat Immunol 9:503-510. 
591. Sun, J. C. and L. L. Lanier. 2011. NK cell development, homeostasis and function: parallels with CD8(+) T cells. Nat Rev Immunol 11:645-657.

592. Chen, Y. J., C. Y. Wu, C. C. Chang, C. J. Ma, M. C. Li, and C. M. Chen. 2008. Nuclear Kruppel-like factor 4 expression is associated with human skin squamous cell carcinoma progression and metastasis. Cancer Biol Ther 7:777-782.

593. Tang, W., Y. Zhu, J. Gao, J. Fu, C. Liu, Y. Liu, C. Song, S. Zhu, Y. Leng, G. Wang, W. Chen, P. Du, S. Huang, X. Zhou, J. Kang, and L. Cui. 2014. MicroRNA-29a promotes colorectal cancer metastasis by regulating matrix metalloproteinase 2 and E-cadherin via KLF4. Br. J Cancer 110:450-458.

594. Vaira, V., A. Faversani, N. M. Martin, D. S. Garlick, S. Ferrero, M. Nosotti, J. L. Kissil, S. Bosari, and D. C. Altieri. 2013. Regulation of lung cancer metastasis by Klf4-Numblike signaling. Cancer Res 73:2695-2705.

595. Shi, Y., L. Ou, S. Han, M. Li, M. M. Pena, E. A. Pena, C. Liu, M. Nagarkatti, D. Fan, and W. Ai. 2014. Deficiency of Kruppel-like factor KLF4 in myeloid-derived suppressor cells inhibits tumor pulmonary metastasis in mice accompanied by decreased fibrocytes. Oncogenesis. 3:e129.

596. Chen, H. Y., Y. M. Lin, H. C. Chung, Y. D. Lang, C. J. Lin, J. Huang, W. C. Wang, F. M. Lin, Z. Chen, H. D. Huang, J. Y. Shyy, J. T. Liang, and R. H. Chen. 2012. miR103/107 promote metastasis of colorectal cancer by targeting the metastasis suppressors DAPK and KLF4. Cancer Res 72:3631-3641.

597. Li, Q., Y. Gao, Z. Jia, L. Mishra, K. Guo, Z. Li, X. Le, D. Wei, S. Huang, and K. Xie. 2012. Dysregulated Kruppel-like factor 4 and vitamin D receptor signaling contribute to progression of hepatocellular carcinoma. Gastroenterology 143:799-810.

598. Yu, F., Y. Shi, J. Wang, J. Li, D. Fan, and W. Ai. 2013. Deficiency of Kruppel-like factor KLF4 in mammary tumor cells inhibits tumor growth and pulmonary metastasis and is accompanied by compromised recruitment of myeloid-derived suppressor cells. Int J Cancer 133:2872-2883.

599. Wei, D., M. Kanai, S. Huang, and K. Xie. 2006. Emerging role of KLF4 in human gastrointestinal cancer. Carcinogenesis 27:23-31. 


\section{CHAPTER 2}

SOX9 inhibits $\beta$-TrCP-mediated protein degradation to promote nuclear GLI1 expression and cancer stem cell properties

Wentao Deng $^{1,2 \dagger}$, Daniel B. Vanderbilt ${ }^{3 \dagger}$, Chen-Chung Lin, Karen H. Martin ${ }^{2}$, Kathleen M. Brundage $^{2}$ and J. Michael Ruppert ${ }^{1-3, *}$

The Department of Biochemistry, ${ }^{1}$ The Mary Babb Randolph Cancer Center, ${ }^{2}$ and Program in Cancer Cell Biology, ${ }^{3}$ West Virginia University, Morgantown, West Virginia 26506.

${ }^{\dagger}$ W.D. and D.B.V. contributed equally to this article.

*Author email for correspondence: mruppert@hsc.wvu.edu 


\begin{abstract}
The High Mobility Group box protein SOX9 and the GLI1 transcription factor play protumorigenic roles in pancreatic ductal adenocarcinoma (PDA). In Kras transgenic mice each of these factors are critical for development of PDA precursor lesions. SOX9 transcription is directly regulated by GLI1, but how SOX9 functions downstream of GLI1 is unclear. We observed positive feedback, such that SOX9-deficient PDA cells have severely repressed levels of endogenous GLI1, attributed to loss of GLI1 protein stability. Sox9 associated with the F-box domain of the SKP1/CUL1/F-box (SCF) E3 ubiquitin ligase component, $\beta$-TrCP, and suppressed its association with SKP1 and GLI1, a substrate of SCF- $\beta$-TrCP. SOX9 also tethered $\beta$-TrCP within the nucleus and promoted its degradation. SOX9 bound to $\beta$-TrCP through its C-terminal PQA/S domain that mediates transcriptional activation. Suppression of $\beta$ - TrCP in SOX9deficient PDA cells restored GLI1 levels and promoted SOX9-dependent cancer stem cell properties. These studies identify SOX9-GLI1 positive feedback as a major determinant of GLI1 protein stability and implicate $\beta$-TrCP as a latent, $\mathrm{SOX} 9$-bound tumor suppressor with the potential to degrade oncogenic proteins in tumor cells.
\end{abstract}




\section{Introduction}

The evolution of metazoans required new strategies for proper regulation of cell fate including intercellular signaling, signal reception, and signal transduction, processes in which transcription factors play critical roles $(1,2)$. Central to cancer etiology is the dysregulation of cell fate, often through genetic changes that impinge on transcription factor signaling $(3,4)$.

GLI1, a member of the GLI family of zinc finger transcription factors, is a central regulator of cell fate that is deregulated in diverse tumor types (5-9). Increased levels of GLI1 mRNA and protein can result from genetic inactivation of tumor suppressors such as the Hedgehog pathway receptor Patched1 (PTCH1), or mutational activation of factors such as Smoothened (SMO). GLI1 signaling impacts multiple cancer-relevant cellular processes, promoting dedifferentiation, the generation of cancer stem cells (CSCs), tumor progression and metastasis. In addition, GLI1 can directly induce the transcription of its own mRNA through a well-characterized, autoregulatory feedback, and therefore GLI1 mRNA levels often reflect the overall GLI transactivation capacity $(10,11)$.

Pancreatic ductal adenocarcinoma (PDA) is an aggressively metastatic tumor type that is often diagnosed at a later clinical stage $(12,13)$. Although GLI1 is expressed in both epithelial PDA cells and stromal cells, a cell autonomous role within carcinoma cells appears central to the pathogenesis of this disease (14-17). Indeed, suppression of GLI1 in human PDA cells leads to loss of malignant properties $(14,16,18)$. In a Kras-dependent mouse model of PDA, either Cremediated excision of Gli1 or expression of a dominant negative GLI factor suppresses tumorigenesis, including the outgrowth of precursor lesions termed pancreatic intraepithelial neoplasia (PanIN) (19,20). Conversely, enforced expression of an active GLI factor in pancreatic 
epithelial cells promotes tumorigenesis in mice (21). In the canonical Hedgehog/GLI pathway, GLI activity is dependent upon signaling by Hedgehog through PTCH and SMO, whereas in PDA cells GLI1 is instead maintained by activated KRAS (15-18,21,22).

The protein stability of GLI1 is regulated by two E3 ubiquitin ligases, the Skp/Cul/F-box complex SCF $^{\beta-\operatorname{TrCP}}$ and NUMB/ITCH $(23,24)$. Similar to slimb regulation of the Drosophila GLI homologue cubitus interruptus, the mammalian $\mathrm{SCF}^{\beta-\operatorname{TrCP}}$ is a major regulator of the protein stability and/or proteolytic cleavage of mammalian GLI1 and its paralogues GLI2 and GLI3 $(25,26) . \mathrm{SCF}^{\beta-\operatorname{TrCP}}$ is comprised of the bridging protein SKP1, the scaffolding protein CUL1, the substrate-recognizing $\mathrm{F}$ box protein $\beta$ - TrCP, and the RING finger protein $\mathrm{RBX} 1$. This complex catalyzes the transfer of ubiquitin from E2 ligase to the substrate, leading to degradation by the ubiquitin proteasome system (UPS) (27). In cultured human keratinocytes, GLI1 stability is dependent upon epidermal growth factor (EGF) signaling through the MEK/ERK pathway (28). Similarly, in cultured human PDA cells, activated KRAS can stabilize the GLI1 protein through ERK1/2 signaling (18). These results suggest a broader role of RAS/MEK/ERK in stabilization of GLI1.

GLI1 directly induces the transcription of SOX9, an Sry-like High Mobility Group (HMG) box transcription factor that plays key roles in sex determination, chondrogenesis and cell differentiation (29-31). Sox9 responds to Hedgehog/Gli signaling in multiple contexts including chondrocytes, retinal progenitor cells, and developing hair follicles (32-35). Consistent with these results the $\operatorname{SOX} 9$ promoter and upstream flanking region contains consensus GLI binding sites that, when linked to a transcriptional reporter, can be regulated by GLI1 in cultured cells $(34,36)$. 
In the developing pancreas, Sox9 is expressed in stem- or progenitor-like cells and is required for normal organogenesis $(37,38)$. In the adult pancreas Sox9 is expressed in ductal and centroacinar cells, but is normally low or absent in acinar cells. Two types of studies have documented a protumorigenic role for SOX9 in PDA. First, xenograft experiments utilizing human PDA cells such as Panc-1 indicate that SOX9 promotes the maintenance of tumorinitiating cells $(34,39)$. Secondly, the induction of PanIN lesions in the conditional Kras ${ }^{\text {G12D }}$ mouse model of PDA involves the early induction of Sox9 in acinar cells, followed by acinarductal metaplasia and tumor progression (40). Conditional gene knockout or enforced expression revealed that Sox9 is critical for the occurrence of PanIN lesions. Similarly SOX9 is protumorigenic in other contexts including colorectal cancer and mammary cancer, promoting the induction of CSC factors such as BMI1 and/or cooperating with mesenchyme-inducing factors such as Snail or Slug $(41,42)$.

In the current study we found that Sox 9 is important for efficient in vitro transformation by GLI1, attributed to its stabilization of GLI1. Like other $\mathrm{SCF}^{\beta-\mathrm{TrCP}}$ substrates, GLI1 interacted with the C-terminal WD domain of $\beta$-TrCP. SOX9 instead interacted with the N-terminal F-box domain, and yet inhibited the association of GLI1 and $\beta$-TrCP. Consistent with a critical role of the SOX9- $\beta$-TrCP interaction for stabilization of GLI1, suppression of $\beta$-TrCP in SOX9deficient PDA cells led to restoration of GLI1 and promoted malignant properties. Because $\mathrm{SCF}^{\beta-\operatorname{TrCP}}$ can promote the ubiquitination of functionally diverse proteins, its ultimate role as provs. anti-tumorigenic may be context-dependent (27,43-45). Our data suggest that $\beta$-TrCPassociated proteins such as SOX9 could be a critical aspect of this context, capable of suppressing SCF activity against multiple pro-tumorigenic substrates such as GLI1, $\beta$-catenin 
and the anti-apoptotic factor MCL1. These results identify reactivation of latent $\beta$ - $\mathrm{TrCP}$ as a strategy for the disruption of KRAS-mediated protumorigenic signaling.

\section{Results}

A role for Sox9 in GLI1-mediated epithelial transformation in vitro. RK3E cells, derived from rat kidney cells by immortalization with adenovirus E1A, undergo malignant transformation in response to GLI1 and provide an epithelial context for functional studies $(46,47)$. Utilizing this model, we identified increased Sox9 mRNA and protein as early responses to exogenous, human GLI1 (HsGli1, Fig. 1A and supplementary material Fig. S1A). In RK3E cells engineered to induce GLI1 when exposed to tetracycline (RK3E-TO GLI1 cells), the Sox9 mRNA was induced between 1 and 3 hours after drug treatment. Kinetics were similar as for other well-established, GLI1-regulated genes including Ptch1 and Bcl2. Consistent with a direct interaction, ChIP analyses and luciferase reporter studies identified a candidate enhancer element containing a GLI1 consensus site, located downstream of the rat Sox 9 coding region and approximately $1.0 \mathrm{~kb}$ downstream of exon 3 (supplementary material Fig. S1B, C, and D).

To analyze Sox 9 we generated shRNA expression vectors ( $\operatorname{sh}$ Sox $9-1$ and -2 ) that stably suppressed the Sox9 mRNA in RK3E cells (Fig. 1B, upper panel). As compared to a nontargeting control (Ctrl), plasmid cotransfection of GLI1 vector with either shRNA construct efficiently inhibited the outgrowth of transformed foci (Fig. 1B, lower panel). In contrast, Sox9 appeared largely dispensable when transformed foci were instead generated using an ERBB2 vector (supplementary material Fig. S1E). 
Following siRNA-mediated suppression of Sox9 in GLI1-transformed RK3E cells, we found that GLI1 protein levels were reduced, suggesting a role for Sox9 in maintenance of GLI1 (Fig. 1C, lanes 2 and 4). Treatment with proteasome inhibitor CL $\beta$ L rapidly restored GLI1 protein levels (Fig. 1D). These results identified Sox9 as a transcription factor with a potential role in GLI1 protein stability.

\section{Endogenous SOX9 stabilizes GLI1 in pancreatic cancer cells through regulation of}

及-TrCP-mediated degradation. Analysis of conditionally-deficient mice has shown that SOX9 and GLI1 play critical roles in PDA development. We analyzed nine PDA tumor cell lines and found that, relative to non-malignant HPDE cells, SOX9 levels were markedly upregulated (range, 10.0- to 240-fold), and most lines likewise expressed increased levels of GLII (Fig. 1E).

To examine a role for endogenous SOX9, we utilized transient or inducible siRNAs that target two distinct sequences (supplementary material Fig. S2A). To detect endogenous GLI1, a low abundance factor, we utilized IP and immunoblot analysis. Using this assay, either of two different GLI1 antibodies identified a protein of $150 \mathrm{kDa}$ (supplementary material Fig. S2B and data not shown). Repression of SOX9 in PDA cells resulted in the reduction of endogenous GLI1 protein levels (Fig. 1F), and mRNA analysis revealed synchronous loss of both SOX9 and GLII signals after siRNA transfection (Fig. 1G).

To analyze how SOX9 regulates GLI1, we engineered PDA cells that independently regulate the GLI1 mRNA and protein, through introduction of a GLI1 transgene under control of a retroviral promoter (Panc-1-GLI1). In these cells the exogenous GLI1 protein was reduced following SOX9 knockdown (Fig. 1H, lanes 3-4). Three independent experiments indicated loss 
of $82 \%$ of the GLI1 (Fig. 1H, bar graph). In contrast, the GLII transgene-derived mRNA levels were not dependent upon SOX9 (Fig. 1H, right panel). In vector control cells the suppression of SOX9 repressed the endogenous GLII mRNA. These results indicate that loss of GLII mRNA following SOX9 suppression is due to destabilization of the GLI1 protein and its autoregulation of GLII transcription.

Interaction of endogenous GLI1 and $\beta$-TrCP proteins was readily detected in human PDA cells (Fig. 1I, left panel). In the absence or presence of proteasome inhibitor the suppression of endogenous SOX9 increased the ratio of $\beta$-TrCP/GLI1 in IPs (Fig. 1I, right panel). Similarly to treatment with proteasome inhibitor, GLI1 expression was rescued by suppression of $\beta$-TrCP (Fig. 1J, lanes 5-6). Similar results were obtained using transient SOX9 siRNA or else the TO conditional approach in either Panc-1 or AsPC-1 cells (supplementary material Fig. S2C).

Sox9 disrupts the interaction of GLI1 with $\beta$-TrCP. Sox9 was previously shown by co-IP and colocalization studies to associate with $\beta$-TrCP, likely through the SOX9 C-terminus, as deletion of this region abrogated binding (48). To investigate whether the association of Sox 9 with $\beta$-TrCP can modulate the targeting of GLI1 for ubiquitination, we utilized HEK293 cells that contain very low levels of endogenous SOX9. When expressed by transient transfection, GLI1 was found to associate with both endogenous and exogenous $\beta-\operatorname{TrCP}$ (Fig. 2A). Similarly as reported (48), the Sox $9 / \beta-\operatorname{TrCP}$ interaction was readily detected (Fig. $2 \mathrm{~B}$ ). However, in multiple experiments we were unable to observe any association of GLI1 and SOX9 (Fig. 2C). Negative results were obtained for co-IP of endogenous proteins in Panc-1 cells as well as overexpressed proteins in HEK293, using both forward and reverse co-IP strategies. These 
results suggested that Sox9 may not regulate GLI1 through a direct interaction but rather could regulate its association with the $\mathrm{E} 3$ ligase component, $\beta$-TrCP.

Consistent with the established role of $\beta$-TrCP in GLI1 regulation, GLI1 levels were suppressed by transient delivery of exogenous $\beta-\mathrm{TrCP}$, and restored by addition of proteasome inhibitor (Fig. 2D). In contrast to GLI1 there was no suppression of Sox9 levels in response to $\beta$ $\operatorname{TrCP}$ (supplementary material Fig. S2D). These results suggested that exogenous Sox9 may prevent the binding of GLI1 to its E3 ligase and the subsequent ubiquitination (23). Indeed, assay of GLI1 stability using the protein synthesis inhibitor cycloheximide (CHX) showed that SOX9 could extend the half-life (Fig. 2E).

Sox9 contains three conserved domains: HMG, PQA and PQS (Fig. 2F). The HMG box mediates DNA binding while PQA and PQS promote transactivation. Deletion analysis indicated that the transactivation region (fragment PQA/S) is sufficient to interact with $\beta$-TrCP, and with comparable efficiency as WT Sox9 (Fig. 2F). As reported (48), deletions within the transactivation domain ( $\Delta \mathrm{C}$ or $\mathrm{HMG})$ abrogated the interaction.

The WD40 repeats of $\beta$-TrCP mediate substrate binding, and the first WD40 repeat plays a critical role in substrate recognition (27). In contrast, the F-box region mediates interaction with SKP1. Consistent with lack of a suppressive effect of $\beta-\operatorname{TrCP}$ on SOX9 (supplementary material Fig. S2D), and unlike $\mathrm{SCF}^{\beta-\mathrm{TrCP}}$ substrates or pseudosubstrates (49), deletion analysis indicated that SOX9 associated with the F-box region of $\beta$-TrCP (Fig. 2G, left panel). As expected for a substrate, GLI1 interacted with the WD40 repeats of $\beta$ - $\mathrm{TrCP}$ (fragment WD1-7), and deletion of the first WD40 repeat (fragment WD2-7) abrogated this interaction (Fig. 2G, 
right panel). Although they interacted with distinct $\beta$-TrCP domains, co-IP of GLI1 and $\beta$-TrCP was nevertheless markedly suppressed in the presence of Sox9 (Fig. 2H, lane 2). In contrast the co-expression of Sox $9 \Delta \mathrm{C}$, deficient for $\beta$-TrCP binding, had little effect (Fig. 2H, lane 3). Unlike for $\beta$-TrCP, using reciprocal co-IPs we observed no interaction of SOX9 with other F-box proteins including FBXW7 and SKP2 (supplementary material Fig. S2E and data not shown).

\section{SOX9 interferes with SKP1 binding and facilitates $\beta$-TrCP turnover in pancreatic}

cancer cells. As SOX9 interacted with F-box region of $\beta$-TrCP, we asked whether this association would interfere with $\beta$-TrCP/SKP1 binding. 293T cells contain much lower levels of endogenous SOX9 compared to Panc-1 cells (Fig. 3A). In 293T cells the binding of Myc- $\beta-\operatorname{TrCP}$ with endogenous SKP1 was suppressed by a mean of $67 \%$ in the presence of exogenous SOX9 (Fig. 3B; three independent experiments). Conversely the binding of Myc- $\beta-\operatorname{TrCP}$ with endogenous SKP1 was increased by a mean of 76\% following SOX9 knockdown in Panc-1 cells (Fig. 3C; three independent experiments). These results identify interference of the $\beta$-TrCPSKP1 association as a mechanism by which SOX9 can stabilize GLI1.

In PDA cells SOX9 suppression resulted in consistent increases in $\beta$ - TrCP protein levels relative to the mRNA (Fig. 3D; see also the lysate panels in Fig. 1I, right panel; Fig. 1J; and supplementary material Fig. S2C). Co-expression of SOX9 with $\beta$-TrCP in HEK293 or Panc-1 cells promoted $\beta$-TrCP degradation (supplementary material Fig. S2D and data not shown). We therefore determined the half-life of SOX9 and $\beta$-TrCP proteins in control PDA cells and in cells deficient in SOX9 or $\beta$-TrCP. Knockdown of SOX9 increased the steady-state abundance of $\beta$ $\operatorname{TrCP}$ and extended its half-life from 140 to 200 minutes (Fig. 3E). In contrast, $\beta$ - $\operatorname{TrCP}$ suppression did not significantly alter the expression or stability of SOX9, which had a half-life 
similar to 230 minutes (Fig. 3F). These results identify distinct inhibitory effects of SOX9 on $\mathrm{SCF}^{\beta-\operatorname{TrCP}}$

\section{SOX9 tethers $\beta$-TrCP within the nucleus and selectively protects nuclear GLI1 from}

degradation. Both SOX9 and $\beta$-TrCP are primarily localized to the cell nucleus $(33,48-50)$. We analyzed the effect of endogenous SOX9 on the localization of transiently-expressed Myc- $\beta$ TrCP in PDA cells. As previously shown for primary chondrocytes (48), SOX9 appeared to efficiently tether $\beta$-TrCP in this compartment (Fig. 4A). In SOX9-deficient PDA cells the $\beta$ $\operatorname{TrCP}$ was instead dispersed throughout the cell such that the nuclear staining was reduced and cytoplasmic staining was increased.

Exogenous GLI1 was detected in both in the nucleus and cytoplasm (Fig. 4B, upper row). In SOX9-deficient cells GLI1 was preferentially lost from the cell nucleus, but cytoplasmic staining was preserved (Fig. 4B, middle row). Consistent with a UPS role in suppression of nuclear GLI1, treatment of cells with CL $\beta \mathrm{L}$ restored nuclear staining (Fig. 4B, lower row). This immunostaining data appeared consistent with the partial suppression of exogenous GLI1 observed by immunoblot analysis (Fig. 1H). The more complete suppression observed for endogenous GLI1 (Fig. 1F, I, J, and supplementary material Fig. S2C) remains unexplained, but could reflect its preferential nuclear localization relative to the overexpressed protein. These results support a role for $\mathrm{SOX} 9$ in tethering of $\beta$-TrCP within the nucleus of PDA cells. Selective loss of nuclear GLI1 upon suppression of SOX9 indicates that nuclear $\mathrm{SCF}^{\beta-\mathrm{TrCP}}$ activity is increased despite the dispersal of $\beta-\operatorname{TrCP}$ throughout the cell. These results suggest that the apparent $\beta-\operatorname{TrCP}$ concentration can be discordant with its activity when SOX9 is present. 


\section{SOX9 promotes the malignant properties of PDA cells in a fashion dependent upon}

$\boldsymbol{\beta}$-TrCP and GLI1. Both SOX9 and GLI1 appear critical for tumorigenesis in PDA $(19,20,40)$. While SOX9-deficient Panc-1 cells fail to form tumors in a mouse xenograft assay, potential links to GLI1 function remain unexplored (34). For SOX9-deficient and control PDA cells we analyzed several in vitro correlates of the malignant phenotype, including proliferation, anchorage-independence, and survival. SOX9-deficient Panc-1 cells proliferated in 2D culture similarly to control cells (Fig. 5A), but were unable to efficiently form colonies in soft agar over a period of 2 weeks (Fig. 5B). Other PDA cell lines, AsPC-1 and MiaPaCa-2, responded similarly. When cells were suspended in low attachment plates for 24 or 48 hours (anoikis assay), deficiency in SOX9 resulted in a marked reduction in the overall viable cell number and the proportion of viable cells (Fig. 5C). These results confirm that SOX9 deficiency can abrogate the malignant properties of PDA cells.

We used the anoikis assay to determine whether deficiency of $\mathrm{SCF}^{\beta-\operatorname{TrCP}}$ substrates such as GLI1 may be responsible for the loss of malignant properties. We restored GLI1 expression in SOX9-deficient cells using $\beta$-TrCP siRNAs (Fig. 5D and supplementary material Fig. S3A). The cell death phenotype of SOX9-deficient PDA cells was attenuated by co-suppression of $\beta$ - $T r C P$ in both Panc-1 and AsPC-1. Consistent with a functional role for GLI1 as a mediator of the increased cell survival following $\beta$-TrCP suppression, co-suppression of GLI1 promoted cell death (Fig. 5E).

\section{SOX9 promotes the cancer stem cell-like properties of PDA cells in a $\beta$-TrCP-}

dependent fashion. CSCs are an aggressively malignant subset of tumor cells (51). In PDA both SOX9 and GLI1 are important for maintenance of this subpopulation $(34,39,52,53)$. To 
determine whether there is phenotypic overlap following modulation of SOX9 or GLI1, we enriched for CSCs using spheroid formation or dye efflux assays (i.e., side population cells, SP) (54) (Fig. 6A). In addition to GLI or GLI-regulated factors (GLII, GLI2, SOX9, SNAII), these subpopulations had increased levels of pancreatic CSC markers (CD24, CD44, ESA, CD133, and CXCR4) as well as factors important for generation of induced pluripotent stem cells (OCT4 and KLF4) (55-57) (Fig. 6B). Consistent with a role for GLI1 in the phenotype of SOX9-deficient cells, suppression of either gene product in 2D cultures of PDA cells reduced the expression of CSC markers, resulting in very similar profiles (Fig. 6C).

We next examined whether $\beta$-TrCP suppression could rescue the CSC phenotype in SOX9-deficient cells. Stable suppression of SOX9 reduced spheroid formation by 75\% (Fig. 6D). Consistent with this result, SOX9 suppression in 2D-cultured PDA cells also reduced the abundance of SP cells (Fig. 6E and supplementary material Fig. S3B). Absent SOX9 suppression, transient suppression of $\beta-\mathrm{TrCP}$ had little effect on spheroid growth (Fig. 6F, left panel). However in SOX9-deficient cells si $\beta$-TrCP partially rescued spheroid formation (Fig. 6F and supplementary material Fig. S3C). The remarkably similar expression profile in SOX9- and GLI1-deficient tumor cells, and restoration of spheroid formation by si $\beta$ - $T r C P$ support a role for suppression of $\mathrm{SCF}^{\beta-\mathrm{TrCP}}$ activity as a mechanism by which SOX9 can promote GLI1 expression and the CSC-like phenotype.

SOX9 is increased while $\beta$-TrCP is decreased in primary human PDA. The above data indicate that SOX9 functions in cultured PDA cells to antagonize $\beta$-TrCP expression and its activity toward GLI1. We utilized Oncomine microarray data (Compendia Bioscience) to analyze SOX9 and $\beta$-TrCP expression in primary human PDA specimens (58). Four studies each 
detected increased levels of PDA signature genes, typified by SFN/14-3-3 $\sigma$, in PDA tumor tissue relative to morphologically normal tissue (59-63) (supplementary material Table S1 and Fig. S4). In these studies SOX9 mRNA expression was upregulated (range, 1.00 to 1.87 fold) while transcripts encoding $\beta$ - TrCP (BTRC) were consistently repressed (range, 1.12 to 1.25 fold). This increase of $S O X 9$ and decrease of BTRC may contribute to the upregulation of GLI1 in PDA $(14,16,64)$.

SOX9 stabilizes other $\boldsymbol{\beta}$-TrCP-regulated proteins. If SOX9 selectively stabilizes nuclear GLI1 by antagonizing $\beta$-TrCP activity in this compartment, then other substrates may be similarly affected, potentially with more subtle effects for cytoplasmic substrates. Consistent with their known regulation by $\mathrm{SCF}^{\beta-\mathrm{TrCP}}, \beta$-catenin, Snail and MCL1 protein levels were each reduced by similar to 50\% following SOX9 knockdown in PDA cells, and CL $\beta$ L treatment restored the levels (Fig. 7A) (65-67). For $\beta$-catenin, consistent results were obtained by transcriptional reporter assay (Fig. 7B) and immunofluorescence analysis (Fig. 7C). Cells transfected with SOX9 siRNA had similar to 50\% reduced expression of both nuclear and cytoplasmic $\beta$-catenin (Fig. 7C and supplementary material Fig. S3D). These data are consistent with a predominant role of SOX9 in the nuclear tethering and suppression of $\mathrm{SCF}^{\beta-\operatorname{TrCP}}$ activity through protein-protein interaction, with more subtle effects in the cytoplasm attributed to alteration of $\beta$ - TrCP concentration (Fig. 4A). Fig. 7D presents a model summarizing the observed effects of SOX9 on $\beta$-TrCP and nuclear GLI1. 


\section{Discussion}

SOX9 is critical for diverse developmental processes including sex determination, chondrogenesis and pancreatogenesis (29-31). For cancers of the prostate, colorectum, pancreas and breast SOX9 plays important roles in carcinoma cell malignant properties $(33,34,39,39-$ 42,68). In chondrocytes SOX9 induces the transcription of genes such as COL2A1, COL11A2 and Aggrecan $(29,69,70)$. Although Sox9-responsive genes were analyzed in several contexts, the mechanisms by which SOX9 promotes malignant properties remain poorly understood, and the relative importance of transcriptional signaling vs. other biochemical activities is unclear $(38,42,71)$.

In rat RK3E epithelial cells we identified Sox9 as an early transcriptional response to GLI1, and linked this regulation to a downstream Sox9 enhancer element using ChIP and transcriptional reporter studies. These data are consistent with previous reports indicating regulation of mouse or human SOX9 by Hedgehog and/or GLI1 (32-35). In analyzing GLI1transformed cells we were surprised to observe a pronounced loss of GLI1 protein stability upon suppression of SOX9, attributed to UPS-mediated degradation. This effect was conserved in human PDA cells and indicated a consistent role for SOX9 in stabilization of GLI1.

In a previous study focused upon chondrocytes, Sox 9 was found to associate with $\beta$-TrCP and to promote $\beta$-TrCP nuclear localization and the degredation of nuclear $\beta$-catenin (48). In the current study focused upon PDA, we found that SOX9 likewise functions as a nuclear tether for $\beta-\operatorname{TrCP}$, but few other aspects of the chondrocyte study appeared to extend to the PDA context. We instead obtained extensive data supporting a potent inhibitory role for SOX9 on $\beta$-TrCP 
activity in the nucleus. Suppression of $\beta$-TrCP in SOX9-deficient cells restored GLI1 levels and promoted phenotypes attributed to SOX9 or GLI1. Supporting the identification of GLI1 as a major factor in the altered phenotype of SOX9-deficient cells, the CSC marker profile of SOX9or GLI1-deficient PDA cells was remarkably similar.

Consistent with an inhibitory effect on $\mathrm{SCF}^{\beta-\operatorname{TrCP}}$, SOX9 interacted through its PQA/S region with the F-box domain of $\beta-\operatorname{TrCP}$, and suppressed the association of $\beta$ - $\operatorname{TrCP}$ with either endogenous or exogenous SKP1. Typical of other substrates, GLI1 associated with the WD domain. Co-immunoprecipitation assays indicated that GLI1 and SOX9 are mutually incompatible for $\beta$-TrCP association. Indeed, in SOX9-deficient PDA cells the association of GLI1 with $\beta$-TrCP was increased by several fold. It is unclear why SOX9 association with the Fbox region would prevent GLI1 from binding to the WD domain. Possibilities include steric hindrance, a conformational shift, or that SOX9 could facilitate the binding of other nuclear proteins such as hnRNP-U, a WD domain-binding pseudosubstrate, and thereby prevent the association of $\beta$-TrCP with GLI1 (49).

An additional effect that strongly supported the inhibition of $\mathrm{SCF}^{\beta-\operatorname{TrCP}}$ by $\mathrm{SOX} 9$ was the destabilization of $\beta-\operatorname{TrCP}$. Whether this instability results from the reduced association with SKP1, from tethering within the nuclear compartment, and/or from some other effect of SOX9 or yet another associated factor is currently unclear.

In SOX9-deficient PDA cells $\beta$-TrCP was more widely distributed throughout the cell, with reduced nuclear staining and similar to a two-fold increase in cytoplasmic staining compared with control cells. This redistribution appeared to impact not only nuclear GLI1 but 
also cytoplasmic proteins such as the $\mathrm{SCF}^{\beta-\mathrm{TrCP}}$ substrate $\beta$-catenin. While nuclear GLI1 was markedly destabilized, the cytoplasmic substrate $\beta$-catenin was suppressed by approximately $50 \%$. Similar effects were observed for two other well-established substrates of SCF ${ }^{\beta-T r C P}$, Snail and MCL1. These results indicate a broad role for SOX9 in the regulation of protein stability.

In SOX9-deficient PDA cells the effects of untethered $\beta$-TrCP were quite distinct for nuclear and cytoplasmic GLI1. These results appear to indicate that nuclear GLI1 is selectively targeted by $\beta$-TrCP, although the mechanism(s) responsible for this selectivity is unclear. The results are overall consistent with identification of the Drosophila homologue as a short-lived nuclear transcriptional activator in response to Hedgehog signaling (72). We conclude that when SOX9 is present the $\beta$-TrCP nuclear staining intensity can be discordant with nuclear $\mathrm{SCF}^{\beta-\operatorname{TrCP}}$ activity.

Mouse model data have established clear roles for GLI1 and SOX9 in the malignant progression of PDA $(14,16,18-21,34,40)$. Interestingly, both SOX9 transcript levels and GLI1 protein stability appear critically dependent upon upstream signaling by RAS/MEK/ERK, but it remains unclear how either factor is regulated by this signaling $(18,28,34,73)$. Whereas a Sox 9 transgene directed to the pancreatic acinar cells is sufficient on its own to initiate early PanIn lesions in mice, a GLI transgene does not induce these lesions, suggesting that GLI is the more dependent factor $(21,40)$. These considerations suggest that KRAS may stabilize GLI1 by signaling through SOX9. Alternatively, it is possible that activated KRAS could more directly regulate GLI1 or both transcription factors in parallel. Blockade of this KRAS signaling in tumor cells, a current focus of research, has the potential to suppress SOX9 and to activate $\beta$-TrCPmediated degradation of multiple oncoproteins. 


\section{Materials and methods}

Expression vectors and plasmid transfection. pcDNA4/TO-HA-GLI1 was described

(47). Retroviral vectors were made by inserting the HAGLI1 cassette of pcDNA4/TO-HA-GLI1 into pB-puro and pLUT7. pLUT7 was provided by Alexey Ivanov (West Virginia University [WVU]). All cloned PCR products were verified by sequencing.

Mouse $\beta-\operatorname{TrCP} 1$ vector containing a $3 \mathrm{xMyc}$ epitope tag at $\mathrm{N}$-terminus (74) was used to generate truncated constructs by PCR. A human ubiquitin vector, Myc-Ub, was provided by Alexey Ivanov. pcDNA3- $\beta$-catenin (Addgene 16828) was a gift from Eric Fearon (75). pCMVMyc CDC4 WT* was a gift from Bert Vogelstein (FBXW7, Addgene plasmid \# 16652). pcDNA3-myc-Skp2 was a gift from Yue Xiong (Addgene plasmid \# 19947).

pTRIPZ-TOshSOX9 lentivector was generated by transfer of the shRNAmir cassette from pGIPZ (V3LHS_396212, Thermo Scientific). Non-targeting TRIPZ lentiviral shRNAmir RHS4743 served as a control. shRNA vectors targeting rat Sox9 (RnSox9) were constructed in pSilencer 2.1-U6 neo as described (47). shRNA target sequences are listed in supplementary material Table S2.

Retroviral transduction was performed as described (46). Transient transfections were performed as described (76) using Lipofectamine 2000 Transfection Reagent (Life Technologies) for Panc-1 cells and TransIT-LT1 Reagent (Mirus) for other cells. 
Isolation of rat Sox9 cDNA and generation of Sox9 expression vectors. PCR was used to synthesize cDNA fragments containing the RnSox9 5' UTR and protein coding regions. The sequence has been deposited to the NCBI (accession pending). pBpuro-RnSox9 was used for stable expression studies. RnSox9 vectors tagged with hemagglutinin (HA), including HA-Sox9 WT and truncations, were generated by PCR followed by insertion into pcDNA3.1.

Cell culture and small molecules. The immortalized human pancreatic ductal epithelial cell line HPDE (H6c7), provided by Ming-Sound Tsao (University of Toronto, Canada), was cultured in keratinocyte serum-free (KSF) medium supplemented with bovine pituitary extract and epidermal growth factor (Life Technologies). S2-013 PDA cells were a gift of Martin Johnson (University of Alabama at Birmingham). S2-013, HEK293, 293T and RK3E cells were cultured in high-glucose DMEM supplemented with L-glutamine, penicillin, streptomycin, and $10 \%$ (v/v) fetal bovine serum. Other PDA cell lines were from American Type Culture Collection (ATCC) and were cultured as recommended by ATCC. Doxycycline (Dox, 0.5 $\mu \mathrm{g} / \mathrm{ml}$ ), tetracycline (tet, $1.0 \mu \mathrm{g} / \mathrm{ml}$ ), cycloheximide (CHX, $50 \mu \mathrm{g} / \mathrm{ml}$ ), clasto-Lactacystin $\beta$ lactone $(\mathrm{CL} \beta \mathrm{L}, 10 \mu \mathrm{M})$, and MG132 $(20 \mu \mathrm{M})$ were from Sigma-Aldrich.

\section{siRNA transfection, RNA isolation and real-time quantitative PCR (qRT-PCR).} siRNA target sequences are listed in supplementary material Table S2. siRNA transfection was performed using Lipofectamine RNAiMax (Life Technologies) as reported (76). For some experiments the Panc-1 cells were re-transfected after 24 hours. Cell extracts were prepared 48 hours after the start of the initial transfection. Co-transfection of plasmids with siRNAs was as reported (76). 
Total RNA was purified using the RNeasy Plus Mini kit (Qiagen). Reverse transcription was performed using SuperScript II (Life Technologies). qRT-PCR reactions utilized Brilliant II SyBr green QPCR master mix, GAPDH served as the internal control, and reactions were analyzed on an Mx3005P system (Agilent). Oligonucleotides are shown in supplementary material Table S3. qRT-PCR results represent three or more independent experiments (bars, s.d.).

Luciferase reporter assay. Topflash (WT TCF4-binding sites) or Fopflash (mutated sites) reporter constructs were gifts of Bert Vogelstein. The Renilla luciferase vector pRL-TK served as an internal control. For siRNA co-transfection, siRNA Ctrl2 served as the control (supplementary material Table S2). Reporter assays were performed on 12-well plates at 48 hours post-transfection using Dual-Luciferase Reporter Assays (Promega) as described (76). Data represent three or more independent experiments (bars, s.d.).

Protein expression studies. For immunoblotting, mouse anti-GLI1 and rabbit anti- $\beta$ TrCP, anti-SKP1 and anti-SNAI1/Snail were from Cell Signaling Technology. Rabbit anti-SOX9 was from EMD Millipore. Mouse anti-Myc (9E10) and anti- $\beta$-catenin were from BD Biosciences. Rat anti-HA (3F10) was from Roche Applied Science. Anti-MCL1 was from Santa Cruz (S-19).

Whole cell lysates for immunoblotting were obtained by extraction in ice-cold RIPA buffer without SDS as described (76). For IP and co-IP, cells were resuspended in NE-A buffer and then lysed by addition of $\mathrm{NaCl}$ and glycerol as described (77). GLI1 protein was precipitated with rabbit anti-GLI1 (47), while rabbit anti-SOX9 (H-90, Santa Cruz Biotechnology) or a 
monoclonal anti-HA (12CA5, Roche) was used for SOX9. Normal rabbit or mouse IgG (SigmaAldrich) was used as control. Protein complexes were recovered using Protein A-Sepharose (Sigma-Aldrich).

For immunoblotting, proteins were transferred to nitrocellulose membrane and detected using chemiluminescence (Pierce ECL, Thermo-Scientific). Quantitative analysis of autoradiographic images was performed using Image J v1.42 (National Institutes of Health), and the normalized results were transferred to GraphPad Prism.

In vitro transformation assay. RK3E transformation assays were performed as described (47). Cells were fixed and stained using Modified Wright Stain (Sigma-Aldrich) at 2-3 weeks post-transfection, and foci with a diameter greater than $1.0 \mathrm{~mm}$ were counted.

Soft Agar Assay. Anchorage-independent cell growth assays were performed on 6-well plates. A $2.0 \mathrm{ml}$ underlay composed of $0.63 \%(\mathrm{w} / \mathrm{v})$ agar in complete DMEM was placed in each well. $1.0 \mathrm{ml}$ of cells $\left(3.0 \times 10^{3}\right)$ in DMEM containing $0.33 \%$ agar was plated on top. Cells were fed with $1.0 \mathrm{ml}$ of $0.63 \%$ agar in DMEM each week. For induction of shRNA expression $0.5 \mu \mathrm{g} / \mathrm{ml}$ of doxycycline was included. Cells were fixed after $2-3$ weeks in $10 \%$ methanol/10\% acetic acid and stained with $0.1 \%$ crystal violet (Sigma-Aldrich) in 50\% methanol. Colonies larger than $50 \mu$ in diameter were counted under phase contrast microscopy.

Anoikis assay. Matrix deprivation assays were performed as described (78). 24 hours post siRNA-transfection, $2.0 \times 10^{4}$ cells were resuspended in $1.0 \mathrm{ml}$ of complete DMEM containing 1\% methylcellulose (Sigma-Aldrich) and plated in 6-well low-attachment plates. 
Assays were performed in triplicate (bars, s.d.). 24 and 48 hours later, cells were collected, resuspended in Accumax (Innovative Cell Technologies) to generate single cell suspensions, and mixed with $0.4 \%$ trypan blue (Cellgro). Trypan blue staining was scored using a hemacytometer.

Spheroid formation Assay. PDA cells were trypsinized, counted, and suspended in 6well, low-attachment plates using 1:1 DMEM/F-12 supplemented with $1 \times \mathrm{B} 27,4.0 \mu \mathrm{g} / \mathrm{ml}$ heparin, $20 \mathrm{ng} / \mathrm{ml}$ EGF, $20 \mathrm{ng} / \mathrm{ml} \mathrm{FGF}$ and 1\% methylcellulose. 2,000-5,000 cells of each sample were plated in triplicate in $2.0 \mathrm{ml}$ final volume. The spheroids in each well were quantitated by phase contrast microscopy. For RNA isolation spheroids were harvested after 7-10 days by centrifugation at $200 \mathrm{Xg}$ for one minute.

Flow cytometry. For side population analysis cells were trypsinized, washed with PBS, and resuspended in staining buffer (DMEM, 2\% [v/v] FBS, $1.0 \mathrm{mM}$ HEPES-KOH, pH 7.0). Samples were incubated for $30 \mathrm{~min}$ at $37^{\circ} \mathrm{C}$ with or without $50 \mu \mathrm{g} / \mathrm{ml}$ of verapamil (SigmaAldrich), mixed with Hoechst 33342 (Sigma-Aldrich) to a final concentration of $5.0 \mu \mathrm{g} / \mathrm{ml}$ and incubated for $90 \mathrm{~min}$ at $37^{\circ} \mathrm{C}$ with intermittent mixing. The cells were washed twice with PBS, resuspended in sorting buffer (1XPBS, 5.0mM EDTA, 1\% [w/v] BSA, 25mM HEPES-KOH, pH 7.0), and maintained at $4^{\circ} \mathrm{C}$ in the dark. Analysis was performed using a FACSAria III cell sorter (BD Biosciences) and both Hoechst Red and Hoechst Blue were measured.

Immunofluorescent staining and quantitation. Cells were treated with CL $\beta L$ or DMSO for 3 hours before fixation. Cells were fixed and stained as described (79) at 48 hours post-transfection using rabbit anti-SOX9 (1:1,000, EMD Millipore), mouse anti-GLI1 (1:100, Cell Signaling), anti-Myc (9E10, $2.5 \mu \mathrm{g} / \mathrm{ml}, \mathrm{BD}$ Biosciences $)$, and/or anti- $\beta$-catenin $(0.17 \mu \mathrm{g} / \mathrm{ml}$, 
BD Biosciences). Secondary antibodies were cross-absorbed Alexa Fluor 647 goat anti-rabbit IgG and Alexa Fluor 555 goat anti-mouse IgG (Life Technologies). Nuclei were counterstained in DAPI prior to mounting of coverslips using ProLong Antifade (Life Technologies).

Digital images were captured and pseudocolored using a Zeiss LSM 510 laser scanning confocal on an upright Zeiss AxioImager and Zeiss LSM software (Carl Zeiss). Images were exported as tiff files and Corel software was used to make minor, identical adjustments to the experimental and control panels in parallel. $\beta$-catenin was quantitated using Image $\mathbf{J}$.

\section{Acknowledgements}

This work was supported by grant NCI RO1 CA127405, the Jo and Ben Statler Chair in Breast Cancer Research and a Pilot grant from the West Virginia University Research Foundation. Flow cytometry experiments were performed in the West Virginia University Flow Cytometry Core Facility, supported by the NIH equipment grant RR020866 and the Institutional Development Award (IDeA) from the National Institute of General Medical Sciences under grants P30GM103488 (CoBRE) and P20GM103434 (INBRE). Imaging experiments and image analysis were performed in the West Virginia University Microscope Imaging Facility, supported by the Mary Babb Randolph Cancer Center and NIH grants P20 RR016440, P30 RR032138/GM103488 and P20 RR016477.

We thank Benoit de Crombrugghe (MD Anderson Cancer Center), Bert Vogelstein (Johns Hopkins University School of Medicine [JHUSOM]), Xu Cao (JHUSOM), Eric Fearon (University of Michigan), Yue Xiong (University of North Carolina at Chapel Hill), and Alexey 
Ivanov (WVU) for providing expression vectors. We thank Ming-Sound Tsao (University of Toronto) and Martin Johnson (University of Alabama at Birmingham) for providing cell lines. 


\section{References}

1. Nusse, R. 2003. Wnts and Hedgehogs: lipid-modified proteins and similarities in signaling mechanisms at the cell surface. Development 130:5297-5305.

2. Weinberg, R. A. 2007. Growth Factors, Receptors, and Cancer, p. 119-158. In R. A. Weinberg (ed.), The Biology of Cancer. Garland Science, Taylor and Francis Group, LLC, New York.

3. Taipale, J. and P. A. Beachy. 2001. The Hedgehog and Wnt signaling pathways in cancer. Nature 411:349-354.

4. Kalderon, D. 2002. Similarities between the Hedgehog and Wnt signaling pathways. Trends Cell Biol 12:523-531.

5. Ruiz i Altaba, A., C. Mas, and B. Stecca. 2007. The Gli code: an information nexus regulating cell fate, stemness and cancer. Trends Cell Biol 17:438-447.

6. Lauth, M. and R. Toftgard. 2007. Non-canonical activation of GLI transcription factors: implications for targeted anti-cancer therapy. Cell Cycle 6:2458-2463.

7. Stecca, B. and A. Ruiz i Altaba. 2010. Context-dependent regulation of the GLI code in cancer by HEDGEHOG and non-HEDGEHOG signals. J Mol. Cell Biol. 2:84-95.

8. Morris, J. P., S. C. Wang, and M. Hebrok. 2010. KRAS, Hedgehog, Wnt and the twisted developmental biology of pancreatic ductal adenocarcinoma. Nat Rev Cancer 10:683695.

9. Hui, C. C. and S. Angers. 2011. Gli proteins in development and disease. Annu. Rev Cell Dev Biol 27:513-537.

10. Dai, P., H. Akimaru, Y. Tanaka, T. Maekawa, M. Nakafuku, and S. Ishii. 1999. Sonic hedgehog-induced activation of the Gli1 promoter is mediated by Gli3. J. Biol. Chem. 274:8143-8152.

11. Vokes, S. A., H. Ji, S. McCuine, T. Tenzen, S. Giles, S. Zhong, W. J. Longabaugh, E. H. Davidson, W. H. Wong, and A. P. McMahon. 2007. Genomic characterization of Gli- 
activator targets in sonic hedgehog-mediated neural patterning. Development 134:19771989.

12. Koorstra, J. B., S. R. Hustinx, G. J. Offerhaus, and A. Maitra. 2008. Pancreatic carcinogenesis. Pancreatology 8:110-125.

13. Feig, C., A. Gopinathan, A. Neesse, D. S. Chan, N. Cook, and D. A. Tuveson. 2012. The pancreas cancer microenvironment. Clin Cancer Res 18:4266-4276.

14. Feldmann, G., S. Dhara, V. Fendrich, D. Bedja, R. Beaty, M. Mullendore, C. Karikari, H. Alvarez, C. Iacobuzio-Donahue, A. Jimeno, K. L. Gabrielson, W. Matsui, and A. Maitra. 2007. Blockade of hedgehog signaling inhibits pancreatic cancer invasion and metastases: a new paradigm for combination therapy in solid cancers. Cancer Res 67:2187-2196.

15. Tian, H., C. A. Callahan, K. J. DuPree, W. C. Darbonne, C. P. Ahn, S. J. Scales, and F. J. de Sauvage. 2009. Hedgehog signaling is restricted to the stromal compartment during pancreatic carcinogenesis. Proc. Natl. Acad. Sci. U. S. A 106:4254-4259.

16. Nolan-Stevaux, O., J. Lau, M. L. Truitt, G. C. Chu, M. Hebrok, M. E. Fernandez-Zapico, and D. Hanahan. 2009. GLI1 is regulated through Smoothened-independent mechanisms in neoplastic pancreatic ducts and mediates PDAC cell survival and transformation. Genes Dev 23:24-36.

17. Lauth, M., A. Bergstrom, T. Shimokawa, U. Tostar, Q. Jin, V. Fendrich, C. Guerra, M. Barbacid, and R. Toftgard. 2010. DYRK1B-dependent autocrine-to-paracrine shift of Hedgehog signaling by mutant RAS. Nat Struct Mol Biol 17:718-725.

18. Ji, Z., F. C. Mei, J. Xie, and X. Cheng. 2007. Oncogenic KRAS activates hedgehog signaling pathway in pancreatic cancer cells. J Biol. Chem. 282:14048-14055.

19. Rajurkar, M., W. E. De Jesus-Monge, D. R. Driscoll, V. A. Appleman, H. Huang, J. L. Cotton, D. S. Klimstra, L. J. Zhu, K. Simin, L. Xu, A. P. McMahon, B. C. Lewis, and J. Mao. 2012. The activity of Gli transcription factors is essential for Kras-induced pancreatic tumorigenesis. Proc Natl Acad Sci U S A 109:E1038-E1047.

20. Mills, L. D., Y. Zhang, R. J. Marler, M. Herreros-Villanueva, L. Zhang, L. L. Almada, F. Couch, C. Wetmore, M. Pasca di Magliano, and M. E. Fernandez-Zapico. 2013. Loss of the transcription factor GLI1 identifies a signaling network in the tumor 
microenvironment mediating KRAS oncogene-induced transformation. J Biol Chem 288:11786-11794.

21. Pasca di Magliano, M., S. Sekine, A. Ermilov, J. Ferris, A. A. Dlugosz, and M. Hebrok. 2006. Hedgehog/Ras interactions regulate early stages of pancreatic cancer. Genes Dev 20:3161-3173.

22. Hingorani, S. R., L. Wang, A. S. Multani, C. Combs, T. B. Deramaudt, R. H. Hruban, A. K. Rustgi, S. Chang, and D. A. Tuveson. 2005. Trp53R172H and KrasG12D cooperate to promote chromosomal instability and widely metastatic pancreatic ductal adenocarcinoma in mice. Cancer Cell 7:469-483.

23. Huntzicker, E. G., I. S. Estay, H. Zhen, L. A. Lokteva, P. K. Jackson, and A. E. Oro. 2006. Dual degradation signals control Gli protein stability and tumor formation. Genes Dev 20:276-281.

24. Di Marcotullio, L., E. Ferretti, A. Greco, S. E. De, A. Po, M. A. Sico, M. Alimandi, G. Giannini, M. Maroder, I. Screpanti, and A. Gulino. 2006. Numb is a suppressor of Hedgehog signalling and targets Gli1 for Itch-dependent ubiquitination. Nat Cell Biol 8:1415-1423.

25. Jiang, J. 2006. Regulation of Hh/Gli signaling by dual ubiquitin pathways. Cell Cycle 5:2457-2463.

26. Huntzicker, E. G. and A. E. Oro. 2008. Controlling hair follicle signaling pathways through polyubiquitination. J Invest Dermatol. 128:1081-1087.

27. Skaar, J. R., J. K. Pagan, and M. Pagano. 2013. Mechanisms and function of substrate recruitment by F-box proteins. Nat Rev Mol Cell Biol 14:369-381.

28. Kasper, M., H. Schnidar, G. W. Neill, M. Hanneder, S. Klingler, L. Blaas, C. Schmid, C. Hauser-Kronberger, G. Regl, M. P. Philpott, and F. Aberger. 2006. Selective modulation of Hedgehog/GLI target gene expression by epidermal growth factor signaling in human keratinocytes. Mol Cell Biol 26:6283-6298.

29. de Crombrugghe, B., V. Lefebvre, and K. Nakashima. 2001. Regulatory mechanisms in the pathways of cartilage and bone formation. Curr Opin Cell Biol 13:721-727. 
30. Barrionuevo, F. and G. Scherer. 2010. SOX E genes: SOX9 and SOX8 in mammalian testis development. Int J Biochem Cell Biol 42:433-436.

31. Kashimada, K. and P. Koopman. 2010. Sry: the master switch in mammalian sex determination. Development 137:3921-3930.

32. Tavella, S., R. Biticchi, A. Schito, E. Minina, D. Di Martino, A. Pagano, A. Vortkamp, W. A. Horton, R. Cancedda, and S. Garofalo. 2004. Targeted expression of SHH affects chondrocyte differentiation, growth plate organization, and Sox9 expression. J Bone Miner. Res 19:1678-1688.

33. Vidal, V. P., M. C. Chaboissier, S. Lutzkendorf, G. Cotsarelis, P. Mill, C. C. Hui, N. Ortonne, J. P. Ortonne, and A. Schedl. 2005. Sox9 is essential for outer root sheath differentiation and the formation of the hair stem cell compartment. Curr. Biol. 15:13401351.

34. Eberl, M., S. Klingler, D. Mangelberger, A. Loipetzberger, H. Damhofer, K. Zoidl, H. Schnidar, H. Hache, H. C. Bauer, F. Solca, C. Hauser-Kronberger, A. N. Ermilov, M. E. Verhaegen, C. K. Bichakjian, A. A. Dlugosz, W. Nietfeld, M. Sibilia, H. Lehrach, C. Wierling, and F. Aberger. 2012. Hedgehog-EGFR cooperation response genes determine the oncogenic phenotype of basal cell carcinoma and tumour-initiating pancreatic cancer cells. EMBO Mol Med 4:218-233.

35. McNeill, B., C. Perez-Iratxeta, C. Mazerolle, M. Furimsky, Y. Mishina, M. A. AndradeNavarro, and V. A. Wallace. 2012. Comparative genomics identification of a novel set of temporally regulated hedgehog target genes in the retina. Mol Cell Neurosci 49:333-340.

36. Bien-Willner, G. A., P. Stankiewicz, and J. R. Lupski. 2007. SOX9cre1, a cis-acting regulatory element located $1.1 \mathrm{Mb}$ upstream of SOX9, mediates its enhancement through the SHH pathway. Hum. Mol. Genet. 16:1143-1156.

37. Seymour, P. A., K. K. Freude, M. N. Tran, E. E. Mayes, J. Jensen, R. Kist, G. Scherer, and M. Sander. 2007. SOX9 is required for maintenance of the pancreatic progenitor cell pool. Proc. Natl. Acad. Sci. U. S. A 104:1865-1870.

38. Lynn, F. C., S. B. Smith, M. E. Wilson, K. Y. Yang, N. Nekrep, and M. S. German. 2007. Sox9 coordinates a transcriptional network in pancreatic progenitor cells. Proc. Natl. Acad. Sci. U. S. A 104:10500-10505. 
39. Sun, L., L. A. Mathews, S. M. Cabarcas, X. Zhang, A. Yang, Y. Zhang, M. R. Young, K. D. Klarmann, J. R. Keller, and W. L. Farrar. 2013. Epigenetic regulation of SOX9 by the NF- $\kappa B$ signaling pathway in pancreatic cancer stem cells. Stem Cells 31:1454-1466.

40. Kopp, J. L., F. G. von, E. Mayes, F. F. Liu, C. L. Dubois, J. P. Morris, F. C. Pan, H. Akiyama, C. V. Wright, K. Jensen, M. Hebrok, and M. Sander. 2012. Identification of Sox9-dependent acinar-to-ductal reprogramming as the principal mechanism for initiation of pancreatic ductal adenocarcinoma. Cancer Cell 22:737-750.

41. Matheu, A., M. Collado, C. Wise, L. Manterola, L. Cekaite, A. J. Tye, M. Canamero, L. Bujanda, A. Schedl, K. S. Cheah, R. I. Skotheim, R. A. Lothe, A. Lopez de Munain, J. Briscoe, M. Serrano, and R. Lovell-Badge. 2012. Oncogenicity of the developmental transcription factor Sox9. Cancer Res 72:1301-1315.

42. Guo, W., Z. Keckesova, J. L. Donaher, T. Shibue, V. Tischler, F. Reinhardt, S. Itzkovitz, A. Noske, U. Zurrer-Hardi, G. Bell, W. L. Tam, S. A. Mani, O. A. van, and R. A. Weinberg. 2012. Slug and Sox9 cooperatively determine the mammary stem cell state. Cell 148:1015-1028.

43. Frescas, D. and M. Pagano. 2008. Deregulated proteolysis by the F-box proteins SKP2 and $\beta$-TrCP: tipping the scales of cancer. Nat. Rev. Cancer 8:438-449.

44. Lau, A. W., H. Fukushima, and W. Wei. 2012. The Fbw7 and $\beta-\operatorname{TrCP}$ E3 ubiquitin ligases and their roles in tumorigenesis. Front Biosci. 17:2197-2212.

45. Shaik, S., C. Nucera, H. Inuzuka, D. Gao, M. Garnaas, G. Frechette, L. Harris, L. Wan, H. Fukushima, A. Husain, V. Nose, G. Fadda, P. M. Sadow, W. Goessling, T. North, J. Lawler, and W. Wei. 2012. SCF ${ }^{\beta-T R C P}$ suppresses angiogenesis and thyroid cancer cell migration by promoting ubiquitination and destruction of VEGF receptor 2. J Exp Med 209:1289-1307.

46. Foster, K. W., S. Ren, I. D. Louro, S. M. Lobo-Ruppert, P. McKie-Bell, W. Grizzle, M. R. Hayes, T. R. Broker, L. T. Chow, and J. M. Ruppert. 1999. Oncogene expression cloning by retroviral transduction of adenovirus E1A-immortalized rat kidney RK3E cells: transformation of a host with epithelial features by c-MYC and the zinc finger protein GKLF. Cell Growth Differ. 10:423-434.

47. Li, X., W. Deng, C. D. Nail, S. K. Bailey, M. H. Kraus, J. M. Ruppert, and S. M. LoboRuppert. 2006. Snail induction is an early response to Gli1 that determines the efficiency of epithelial transformation. Oncogene 25:609-621. 
48. Topol, L., W. Chen, H. Song, T. F. Day, and Y. Yang. 2009. Sox9 inhibits Wnt signaling by promoting $\beta$-catenin phosphorylation in the nucleus. J Biol. Chem. 284:3323-3333.

49. Davis, M., A. Hatzubai, J. S. Andersen, E. Ben-Shushan, G. Z. Fisher, A. Yaron, A. Bauskin, F. Mercurio, M. Mann, and Y. Ben-Neriah. 2002. Pseudosubstrate regulation of the $\mathrm{SCF}^{\beta-\mathrm{TrCP}}$ ubiquitin ligase by hnRNP-U. Genes Dev 16:439-451.

50. Wang, H., N. C. McKnight, T. Zhang, M. L. Lu, S. P. Balk, and X. Yuan. 2007. SOX9 is expressed in normal prostate basal cells and regulates androgen receptor expression in prostate cancer cells. Cancer Res 67:528-536.

51. Clevers, H. 2011. The cancer stem cell: premises, promises and challenges. Nat Med 17:313-319.

52. Tang, S. N., J. Fu, D. Nall, M. Rodova, S. Shankar, and R. K. Srivastava. 2012. Inhibition of sonic hedgehog pathway and pluripotency maintaining factors regulate human pancreatic cancer stem cell characteristics. Int J Cancer 131:30-40.

53. Li, S. H., J. Fu, D. N. Watkins, R. K. Srivastava, and S. Shankar. 2013. Sulforaphane regulates self-renewal of pancreatic cancer stem cells through the modulation of Sonic hedgehog-GLI pathway. Mol Cell Biochem 373:217-227.

54. Bhagwandin, V. J. and J. W. Shay. 2009. Pancreatic cancer stem cells: fact or fiction? Biochim. Biophys. Acta 1792:248-259.

55. Takahashi, K. and S. Yamanaka. 2006. Induction of pluripotent stem cells from mouse embryonic and adult fibroblast cultures by defined factors. Cell 126:663-676.

56. Li, C., D. G. Heidt, P. Dalerba, C. F. Burant, L. Zhang, V. Adsay, M. Wicha, M. F. Clarke, and D. M. Simeone. 2007. Identification of pancreatic cancer stem cells. Cancer Res 67:1030-1037.

57. Hermann, P. C., S. L. Huber, T. Herrler, A. Aicher, J. W. Ellwart, M. Guba, C. J. Bruns, and C. Heeschen. 2007. Distinct populations of cancer stem cells determine tumor growth and metastatic activity in human pancreatic cancer. Cell Stem Cell 1:313-323.

58. Rhodes, D. R., J. Yu, K. Shanker, N. Deshpande, R. Varambally, D. Ghosh, T. Barrette, A. Pandey, and A. M. Chinnaiyan. 2004. ONCOMINE: a cancer microarray database and integrated data-mining platform. Neoplasia 6:1-6. 
59. Logsdon, C. D., D. M. Simeone, C. Binkley, T. Arumugam, J. K. Greenson, T. J. Giordano, D. E. Misek, R. Kuick, and S. Hanash. 2003. Molecular profiling of pancreatic adenocarcinoma and chronic pancreatitis identifies multiple genes differentially regulated in pancreatic cancer. Cancer Res 63:2649-2657.

60. Badea, L., V. Herlea, S. O. Dima, T. Dumitrascu, and I. Popescu. 2008. Combined gene expression analysis of whole-tissue and microdissected pancreatic ductal adenocarcinoma identifies genes specifically overexpressed in tumor epithelia. Hepatogastroenterology 55:2016-2027.

61. Iacobuzio-Donahue, C. A., A. Maitra, M. Olsen, A. W. Lowe, N. T. van Heek, C. Rosty, K. Walter, N. Sato, A. Parker, R. Ashfaq, E. Jaffee, B. Ryu, J. Jones, J. R. Eshleman, C. J. Yeo, J. L. Cameron, S. E. Kern, R. H. Hruban, P. O. Brown, and M. Goggins. 2003. Exploration of global gene expression patterns in pancreatic adenocarcinoma using cDNA microarrays. Am J Pathol 162:1151-1162.

62. Pei, H., L. Li, B. L. Fridley, G. D. Jenkins, K. R. Kalari, W. Lingle, G. Petersen, Z. Lou, and L. Wang. 2009. FKBP51 affects cancer cell response to chemotherapy by negatively regulating Akt. Cancer Cell 16:259-266.

63. Segara, D., A. V. Biankin, J. G. Kench, C. C. Langusch, A. C. Dawson, D. A. Skalicky, D. C. Gotley, M. J. Coleman, R. L. Sutherland, and S. M. Henshall. 2005. Expression of HOXB2, a retinoic acid signaling target in pancreatic cancer and pancreatic intraepithelial neoplasia. Clin Cancer Res 11:3587-3596.

64. Thayer, S. P., M. Pasca di Magliano, P. W. Heiser, C. M. Nielsen, D. J. Roberts, G. Y. Lauwers, Y. P. Qi, S. Gysin, C. C. Fernandez-del, V. Yajnik, B. Antoniu, M. McMahon, A. L. Warshaw, and M. Hebrok. 2003. Hedgehog is an early and late mediator of pancreatic cancer tumorigenesis. Nature 425:851-856.

65. Polakis, P. 1999. The oncogenic activation of beta-catenin. Curr Opin Genet Dev 9:1521.

66. Zhou, B. P., J. Deng, W. Xia, J. Xu, Y. M. Li, M. Gunduz, and M. C. Hung. 2004. Dual regulation of Snail by GSK-3 $\beta$-mediated phosphorylation in control of epithelialmesenchymal transition. Nat. Cell Biol. 6:931-940.

67. Ding, Q., X. He, J. M. Hsu, W. Xia, C. T. Chen, L. Y. Li, D. F. Lee, J. C. Liu, Q. Zhong, X. Wang, and M. C. Hung. 2007. Degradation of Mcl-1 by $\beta$-TrCP mediates glycogen 
synthase kinase 3-induced tumor suppression and chemosensitization. Mol Cell Biol 27:4006-4017.

68. Wang, H., I. Leav, S. Ibaragi, M. Wegner, G. F. Hu, M. L. Lu, S. P. Balk, and X. Yuan. 2008. SOX9 is expressed in human fetal prostate epithelium and enhances prostate cancer invasion. Cancer Res 68:1625-1630.

69. Oh, C. D., S. N. Maity, J. F. Lu, J. Zhang, S. Liang, F. Coustry, B. de Crombrugghe, and H. Yasuda. 2010. Identification of SOX9 interaction sites in the genome of chondrocytes. PLoS One 5:e10113.

70. Pritchett, J., V. Athwal, N. Roberts, N. A. Hanley, and K. P. Hanley. 2011. Understanding the role of SOX9 in acquired diseases: lessons from development. Trends Mol Med 17:166-174.

71. Bhandari, R. K., M. M. Haque, and M. K. Skinner. 2012. Global genome analysis of the downstream binding targets of testis determining factor SRY and SOX9. PLoS One 7:e43380.

72. Ohlmeyer, J. T. and D. Kalderon. 1998. Hedgehog stimulates maturation of cubitus interruptus into a labile transcriptional activator. Nature 396:749-753.

73. Murakami, S., M. Kan, W. L. McKeehan, and B. de Crombrugghe. 2000. Up-regulation of the chondrogenic Sox 9 gene by fibroblast growth factors is mediated by the mitogenactivated protein kinase pathway. Proc Natl Acad Sci U S A 97:1113-1118.

74. Wan, M., Y. Tang, E. M. Tytler, C. Lu, B. Jin, S. M. Vickers, L. Yang, X. Shi, and X. Cao. 2004. Smad4 protein stability is regulated by ubiquitin ligase $S C F^{\beta-\operatorname{TrCP} 1}$. J Biol. Chem. 279:14484-14487.

75. Kolligs, F. T., G. Hu, C. V. Dang, and E. R. Fearon. 1999. Neoplastic transformation of RK3E by mutant $\beta$-catenin requires deregulation of Tcf/Lef transcription but not activation of c-myc expression. Mol. Cell. Biol. 19:5696-5706.

76. Lin, C. C., L. Z. Liu, J. B. Addison, A. V. Ivanov, and J. M. Ruppert. 2011. A KLF4miRNA-206 autoregulatory feedback loop can promote or inhibit protein translation depending upon cell context. Mol. Cell Biol. 31:2513-2527. 
77. Chen, X. and J. J. Bieker. 2001. Unanticipated repression function linked to erythroid Kruppel-like factor. Mol. Cell Biol. 21:3118-3125.

78. Kumar, S., S. H. Park, B. Cieply, J. Schupp, E. Killiam, F. Zhang, D. L. Rimm, and S. M. Frisch. 2011. A pathway for the control of anoikis sensitivity by E-cadherin and epithelial-to-mesenchymal transition. Mol Cell Biol 31:4036-4051.

79. Pandya, A. Y., L. I. Talley, A. R. Frost, T. J. Fitzgerald, V. Trivedi, M. Chakravarthy, D. C. Chhieng, W. E. Grizzle, J. A. Engler, H. Krontiras, K. I. Bland, A. F. Lobuglio, S. M. Lobo-Ruppert, and J. M. Ruppert. 2004. Nuclear localization of KLF4 is associated with an aggressive phenotype in early-stage breast cancer. Clin. Cancer Res. 10:2709-2719. 
FIG. 1. SOX9 stabilizes GLI1 in the rat RK3E epithelial model and in human PDA cells. (A) Rapid induction of SOX9 by GLI1. RK3E-derived, tet-on (TO) GLI1 cells were induced with tet or with vehicle control, and gene expression was analyzed by qRT-PCR ( N=4; column, mean; bars, s.d.). (B) Role of Sox9 for GLI1-mediated in vitro transformation. Upper panel: Sox9 shRNA constructs were transfected into RK3E cells. Sox 9 mRNA was analyzed by qRTPCR following drug selection. Lower panel: GLI1-mediated in vitro transformation was assayed in RK3E cells by counting morphologically-transformed foci. Dishes are representative of three independent experiments. Background was determined using empty vector and was $<1$ focus per dish (not shown). (C) Dependence of GLI1 protein expression on Sox9. GLI1-transformed RK3E cells were transfected with the indicated siRNA. Protein expression was analyzed at $48 \mathrm{~h}$ posttransfection (asterisk, nonspecific). (D) Proteasome inhibition restores GLI1 in SOX9-deficient cells. GLI1-transformed RK3E cells were transfected with Sox9 siRNA. Cells were treated with vehicle (DMSO, 1h) or with proteasome inhibitor (CL $\beta \mathrm{L})$ for the indicated interval. Cell extracts for protein analysis were prepared at $48 \mathrm{~h}$ post-transfection. (E) SOX9 and GLI1 were analyzed in human PDA cell lines. mRNA levels were normalized to immortalized pancreatic ductal epithelial cells (HPDE). (F) Dependence of GLI1 protein expression on SOX9. Panc-1 cells were treated with control or SOX9 siRNA, and endogenous GLI1 protein levels were determined by IP-immunoblot analysis. (G) Dependence of GLI1 mRNA expression on SOX9. Panc-1 cells were treated with siRNA and SOX9 and GLII mRNA levels were assessed at the indicated posttransfection interval. (H) SOX9 can regulate GLI1 protein levels independently of the mRNA. To circumvent the GLI1 autoregulation of its own transcription, a GLI1 retroviral vector was transduced into Panc-1 cells such that the preponderance of GLII mRNA was transcribed under control of the LTR promoter (Panc-1-GLI1) vs. the endogenous GLII promoter (Panc-1-pBabe). 
The impact of SOX9 deficiency on GLI1 protein level in Panc-1-GLI1 cells was then analyzed by immunoblot (left panel). Following normalization to $\beta$-actin, the GLI1 protein level in control and SOX9-deficient cells was quantitated for three independent assays (middle panel). Similarly, mRNA levels were analyzed by qRT-PCR (right panel). (I) SOX9 regulates the association of GLI1 and $\beta$-TrCP. Left panel, endogenous GLI1/ $\beta$-TrCP interaction in Panc-1 cells was determined by Co-IP-immunoblot analysis. Right panel, Panc-1 cells were treated with control siRNA, SOX9 siRNA, and/or CL $\beta L$ as indicated; endogenous GLI1/ $\beta$-TrCP interaction was evaluated by Co-IP-immunoblot analysis. (J) Role of $\beta$-TrCP and the proteasome in the regulation of GLI1 by SOX9. Panc-1 cells were treated as indicated and endogenous protein levels were determined by IP-immunoblot analysis. *, $\mathrm{P}<0.05$; **, $\mathrm{P}<0.01$; ***, $\mathrm{P}<0.001$; ns, not significant.

FIG. 2. Sox9 and GLI1 interact with distinct regions of $\beta$-TrCP in a mutually incompatible fashion. Enforced expression studies were performed in HEK293 cells (A-H). (A) Co-IP analysis of GLI1 and $\beta-\operatorname{TrCP}$. Immunoprecipitated proteins were analyzed by immunoblot analysis. Lysate lanes represent 5\% of the input extract. (B) Co-IP analysis of Sox 9 and $\beta$-TrCP. (C) Co-IP analysis of GLI1 and SOX9. (D) Role of the proteasome in the regulation of GLI1 by $\beta$-TrCP. Cells were transfected with indicated vectors and treated with proteasome inhibitor (MG132) or vehicle (DMSO) for 6 hours prior to preparation of cell extracts for immunoblot analysis. (E) Stabilization of GLI1 by SOX9. Cells transfected with indicated vectors were treated with cycloheximide (CHX) prior to preparation of cell extracts for immunoblot analysis. (F) Co-IP analysis of Myc- $\beta$-TrCP with wild-type or truncated HA-Sox9 constructs. Precipitated proteins were detected by immunoblot. (G) SOX9 and GLI1 interact with distinct regions of $\beta$ TrCP. Co-IP analysis of GLI1 or SOX9 with wild-type or truncated Myc- $\beta$-TrCP constructs. 
Precipitated proteins were detected by immunoblot analysis (asterisk, nonspecific species). (H)

The Sox9 C-terminal region is required for disruption of the GLI1 interaction with $\beta$-TrCP. Cells stably transduced with a GLI1 retroviral vector were transfected with the indicated expression vectors. Myc- $\beta$-TrCP plasmid amount were doubled when co-transfected with wild type Sox 9 (middle lane) to achieve similar Myc- $\beta$-TrCP protein levels. Cells were treated with MG132 for 3 hours before they were harvested for Co-IP analysis. Similar results were obtained in an independent experiment.

FIG. 3. SOX9 disrupts the $\beta$-TrCP/SKP1 association and destabilizes $\beta$-TrCP in PDA cells. (A) Relative levels of SOX9, $\beta$-TrCP, and SKP1 protein levels in HEK293T and Panc-1 cells. (B) Effect of exogenous SOX9 on the association of SKP1 and $\beta$-TrCP. 293T cells were transfected with the indicated expression vectors. Myc- $\beta$-TrCP plasmid amounts were doubled when co-transfected with wild type SOX9 to achieve similar Myc- $\beta$-TrCP protein expression. Cells were treated with MG132 for 3 hours before they were harvested for Co-IP analysis. The results of three independent experiments are indicated (bars, s.d.). (C) Effect of endogenous SOX9 on the association of SKP1 and $\beta$-TrCP. Panc-1 cells were transfected with the indicated expression vectors and siRNAs. Cells were treated with MG132 for 3 hours before harvested for Co-IP analysis. The results of three independent experiments are indicated. (D) Effect of SOX9 on expression of $\beta$-TrCP. Panc-1 cells were treated with the indicated siRNA and then analyzed by immunoblot analysis (left panel) or by qRT-PCR (right panel). (E-F) Analyses of protein stability. Panc-1 cells were transfected with the indicated siRNA and then treated with cycloheximide (CHX) for the indicated interval prior to preparation of extracts for immunoblot analysis at $48 \mathrm{~h}$ post-transfection. The scanned images were quantitated using NIH Image J and 
normalized to $\beta$-actin. Rate constants and protein half-lives were determined using the first order rate law.

FIG. 4. SOX9 tethers $\beta$-TrCP within the nuclei of PDA cells and protects nuclear GLI1 from degradation. (A) Panc-1 cells were transfected with exogenous, Myc epitope-tagged, $\beta$ TrCP together with indicated siRNA. (B) Panc-1 cells were infected with lentiviral GLI1 vector and then with the indicated siRNA. Cells were treated with CL $\beta \mathrm{L}$ or vehicle (DMSO) for the final 3 hours prior to fixation. Cells were fixed and processed for indirect immunofluorescence analysis at 48 hours posttransfection. Scale bar, $10 \mu$.

FIG. 5. In a GLI1-dependent fashion, $\beta$-TrCP suppression rescues malignant properties in SOX9-deficient PDA cells. (A) Panc-1 cell growth in 2D culture was analyzed following transfection of SOX9 or control siRNAs (upper panel). Alternatively, Panc-1-TO shRNA cells were treated with Dox (lower panel). (B) Analysis of anchorage-independent growth regulation

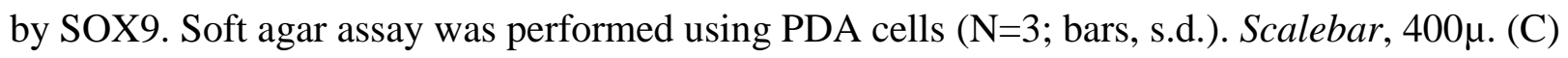
Analysis of anoikis regulation by SOX9. Anoikis assay was performed using Panc-1 cells. The number of surviving cells (left $\mathrm{Y}$-axis) and the percentages of viable cells (right $\mathrm{Y}$-axis) are indicated for each timepoint ( $\mathrm{N}=3$; bars, s.d.). (D-E) Role of $\beta$ - TrCP in the phenotype of SOX9deficient cells. Following siRNA transfection, anoikis assays were performed in Panc-1 cells.

FIG. 6. $\beta$-TrCP suppression rescues CSC properties in SOX9-deficient PDA cells. (A) Panc-

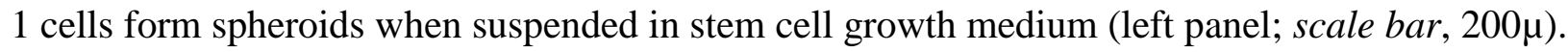
Side population (SP) Panc-1 cells were identified by verapamil-sensitive dye efflux (right panel). (B) Expression profiling of spheroid cells and SP cells. (C) Roles of SOX9 and GLI1 in the 
regulation of CSC markers. The expression of putative CSC markers was determined in Panc-1 cells treated with the indicated siRNA. (D) Spheroid formation assay of Panc-1-TO shRNA cells (N=3; bars, s.d.). (E) SP analysis of PDA cells. Cells were treated with Dox or vehicle for five days and then stained with Hoechst 33342. The results of three independent experiments are presented (right panel). (F) Role of $\beta$-TrCP in the growth of tumor spheroids. si $\beta$-TrCP had little effect on its own in parental Panc-1 cells (left panel). The effect of si $\beta$-TrCP was then analyzed in the context of SOX9-deficient PDA cells (middle and right panels).

FIG. 7. SOX9 stabilizes $\boldsymbol{\beta}$-catenin in Panc-1 cells. (A) Regulation of diverse SCF ${ }^{\beta-\operatorname{TrCP}}$ substrates by SOX9. Panc-1 cells were treated with the indicated siRNA and protein expression was analyzed by immunoblot. (B) Regulation of $\beta$-catenin activity by SOX9 and $\beta$-TrCP. Panc-1 cells were transfected with the indicated siRNA and TCF4-dependent transcriptional activity was determined by luciferase reporter assay. (C) Regulation of $\beta$-catenin protein expression by SOX9. Panc-1 cells were treated with the indicated siRNA and then analyzed by indirect immunofluorescence analysis. Scale bar, $10 \mu$. Quantitation of $\beta$-catenin is shown in Fig. S3D in the supplemental material. (D) A model depicting the effects of SOX9 on $\beta$-TrCP in PDA cells, including nuclear tethering (1), disruption of its association with Gli1 (2), promotion of UPSmediated turnover (3) and disruption of its association with SKP1 (4). 


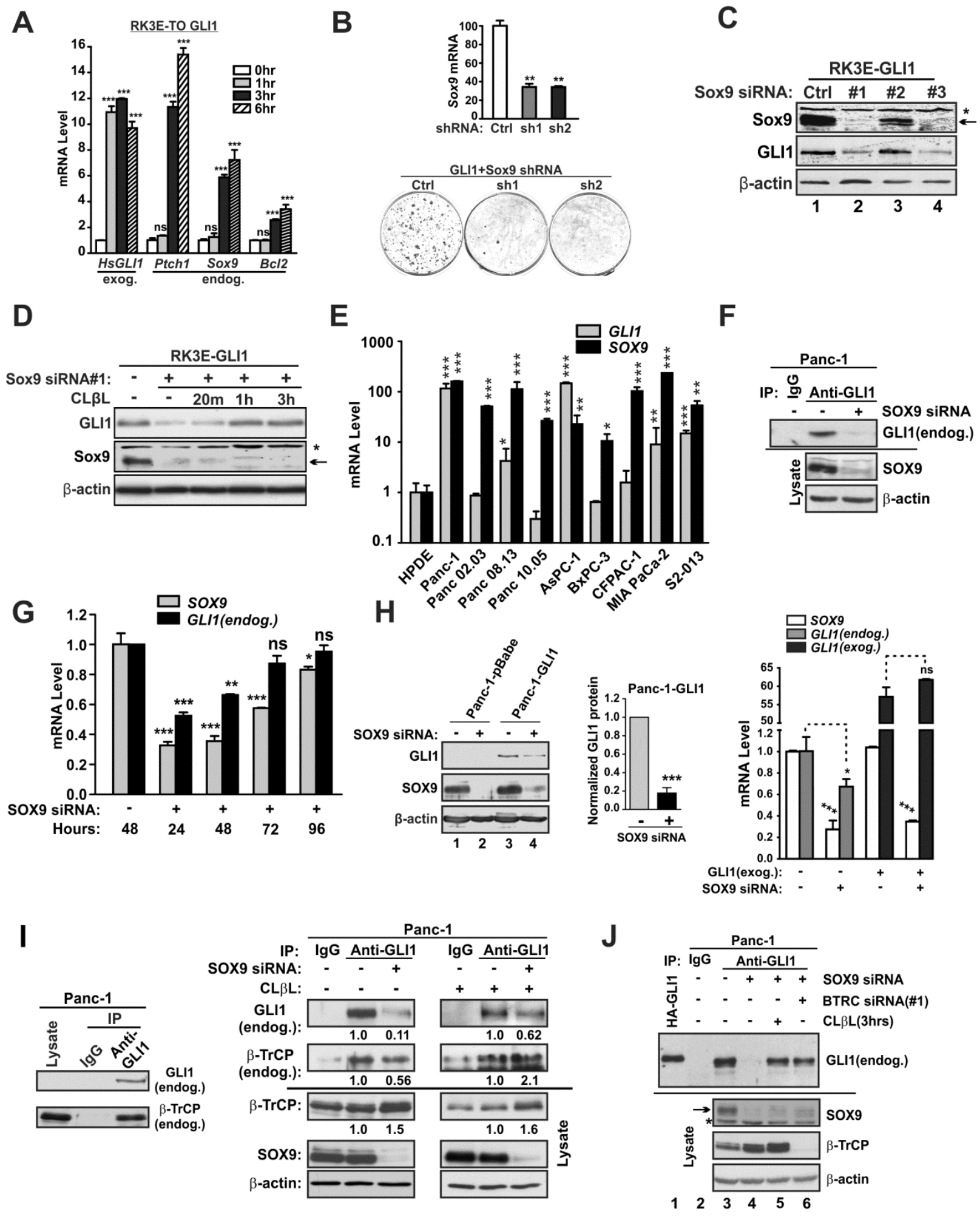

Figure 1. SOX9 stabilizes GLI1 in the rat RK3E epithelial model and in human PDA cells. 

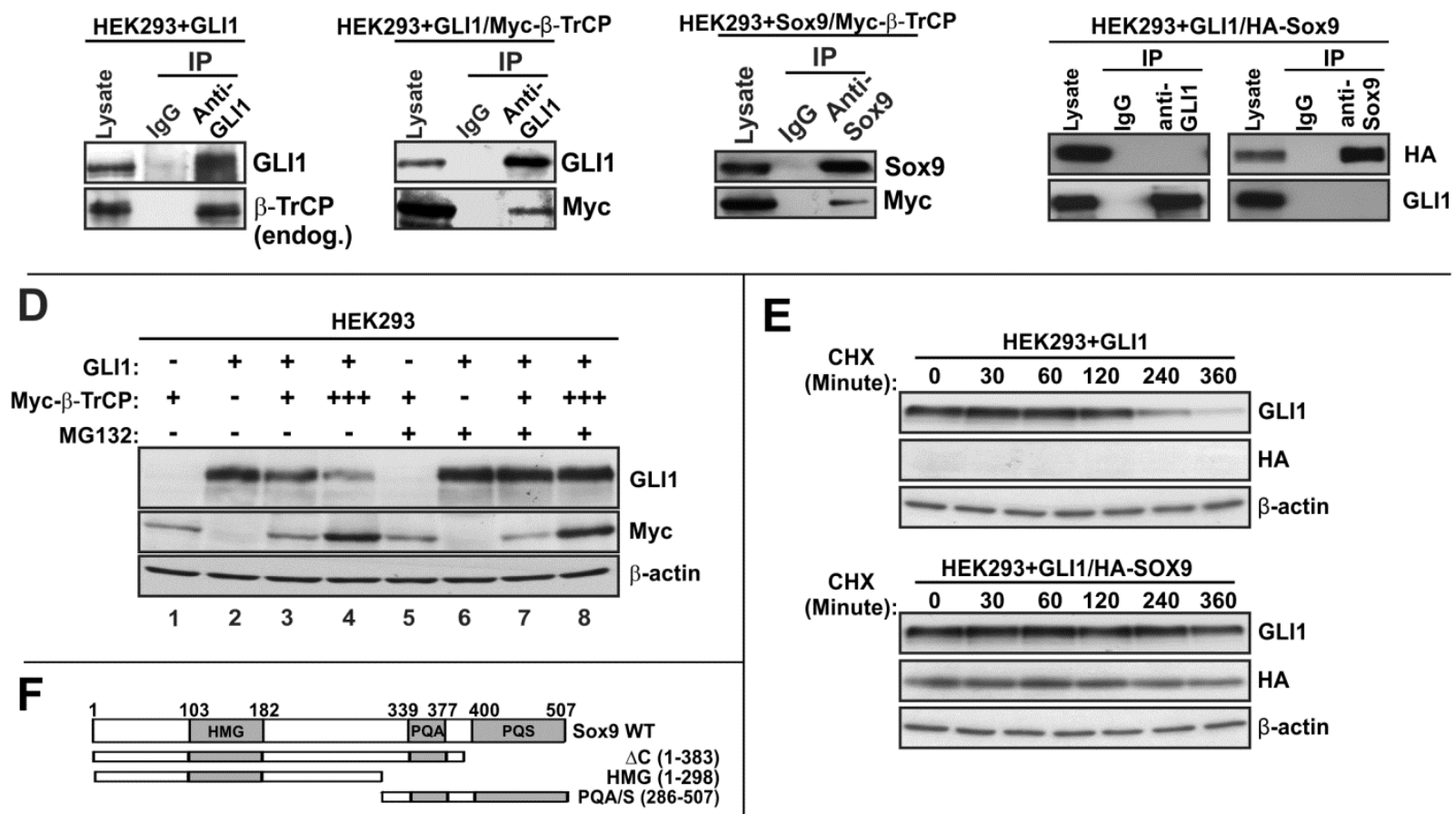

HA-Sox9: - WT $\triangle \mathrm{C}$ HMG PQA/S

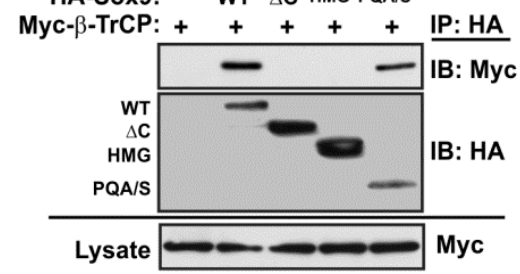

\section{H}
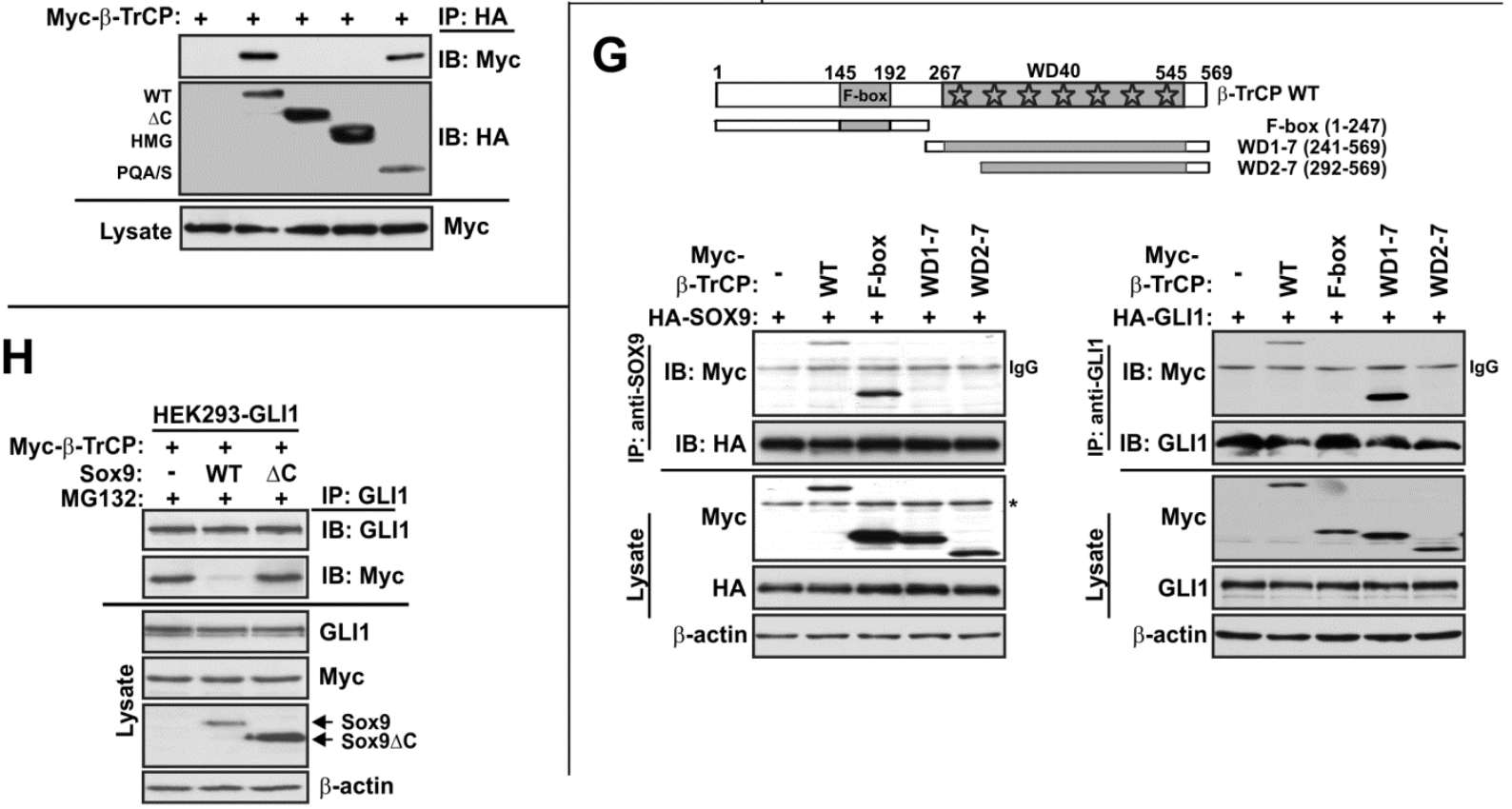

Figure 2. Sox 9 and GLI1 interact with distinct regions of $\beta$-TrCP in a mutually incompatible fashion. 
A

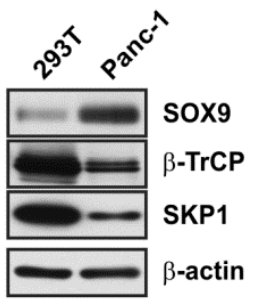

B
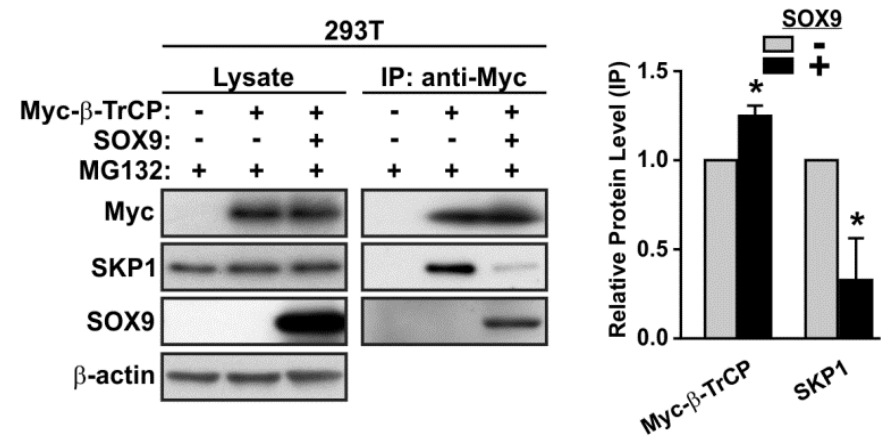

C

Panc-1
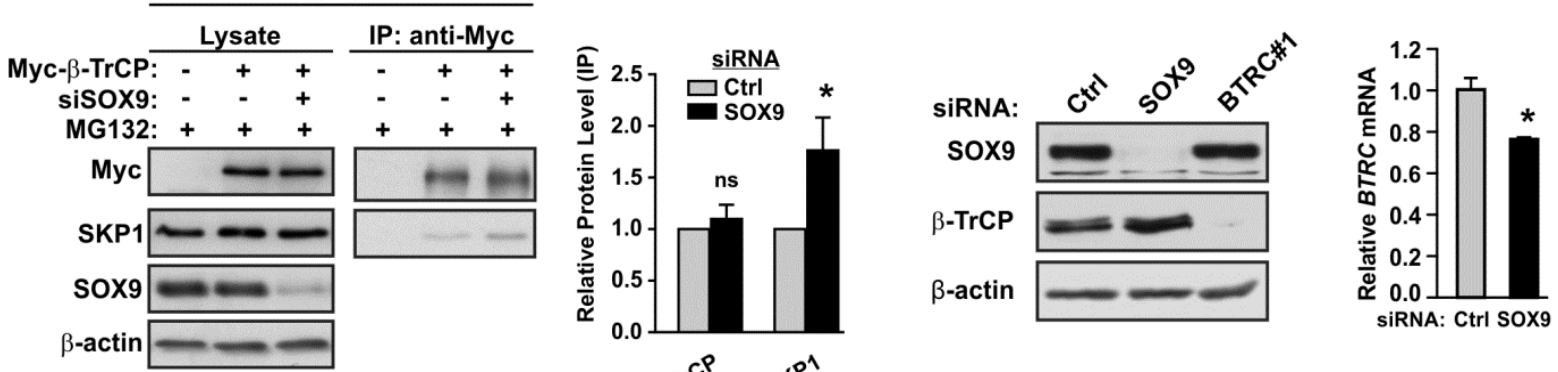

D

E
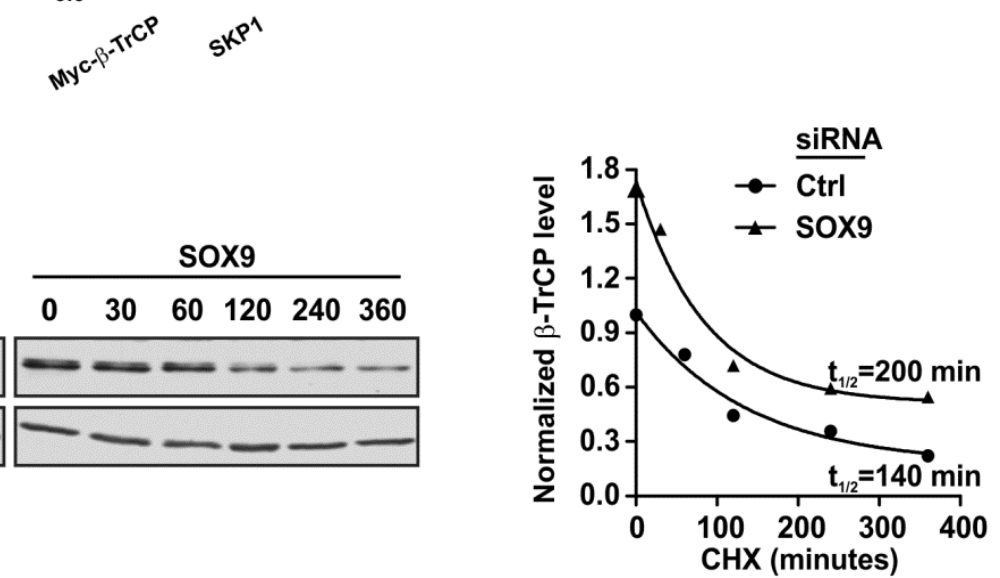

$\mathbf{F}$

\begin{tabular}{|c|c|c|c|c|c|c|c|c|c|c|c|c|}
\hline siRNA & & & C & trl & & & & & BTR & C\#1 & & \\
\hline HX(Minute) & : 0 & 30 & 60 & 120 & 240 & 360 & 0 & 30 & 60 & 120 & 240 & 360 \\
\hline Sox9 & $=$ & - & - & - & $\omega$ & $=$ & $=$ & $=$ & $=$ & $=$ & $=$ & $=$ \\
\hline$\beta$-actin & $\longrightarrow$ & $\ldots$ & 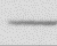 & 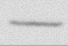 & 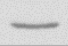 & - & - & $\longrightarrow$ & 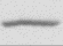 & - & - & - \\
\hline
\end{tabular}

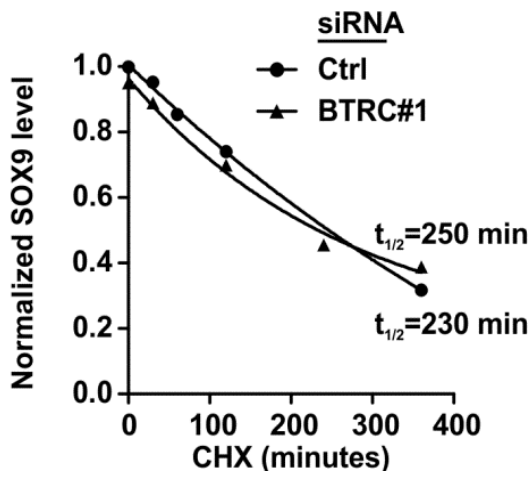

Figure 3. SOX9 disrupts the $\beta$-TrCP/SKP1 association and destabilizes $\beta$-TrCP in PDA cells. 
A

Panc-1+Myc- $\beta$-TrCP

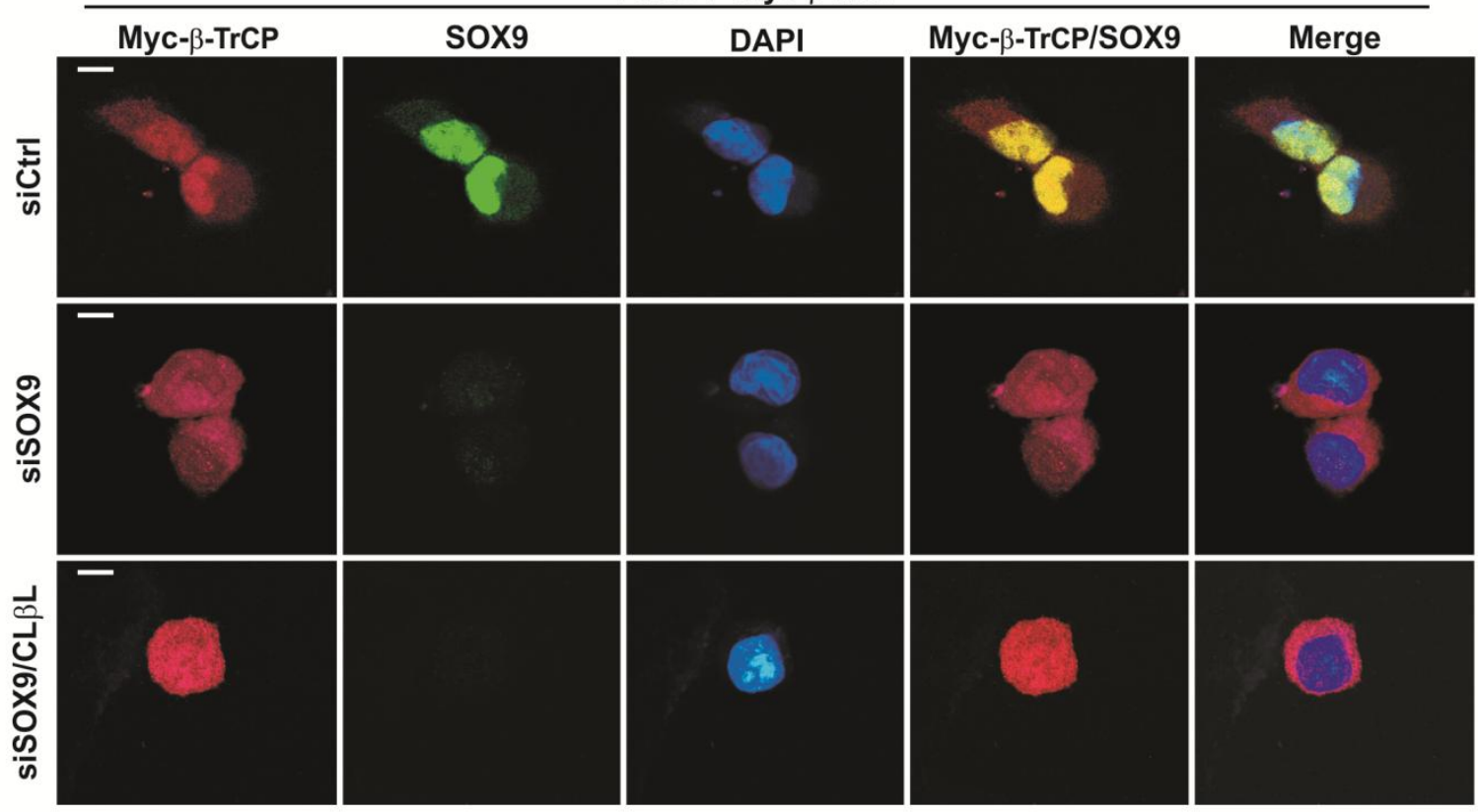

B

Panc-1-GLI1

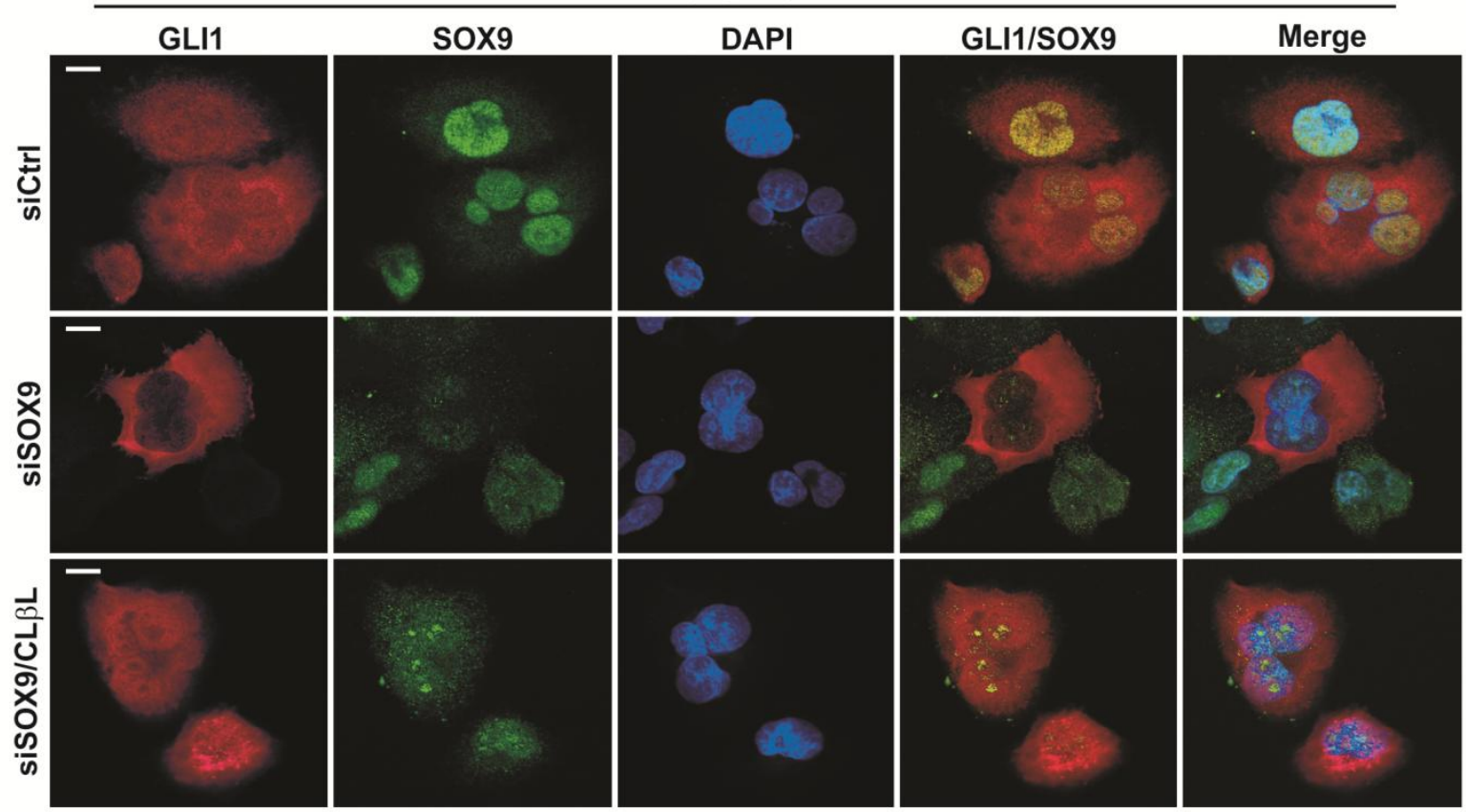

Figure 4. SOX9 tethers $\beta$-TrCP within the nuclei of PDA cells and protects nuclear GLI1 from degradation. 
A
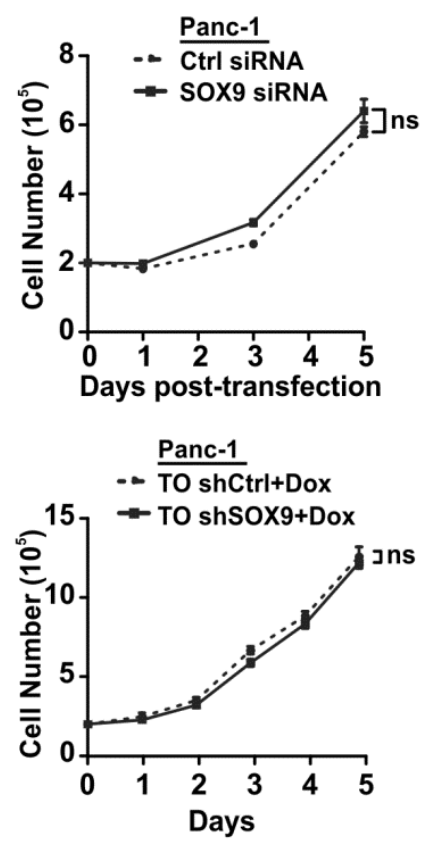

C

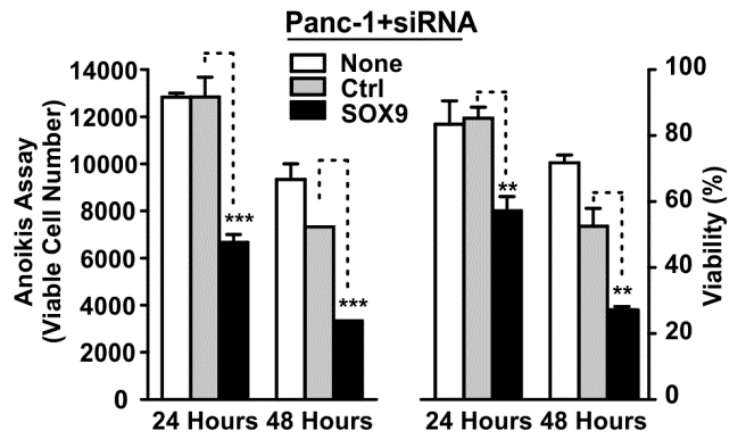

B
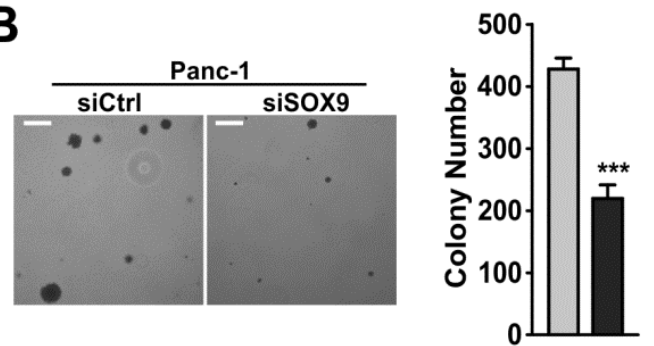

siRNA: Ctrl SOX9
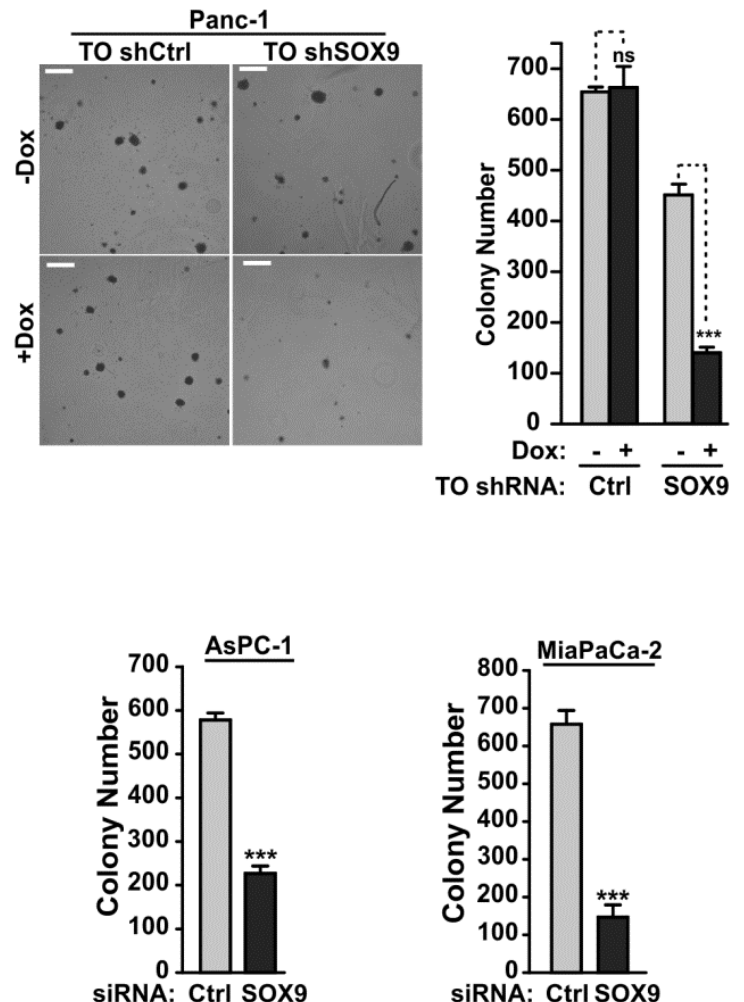

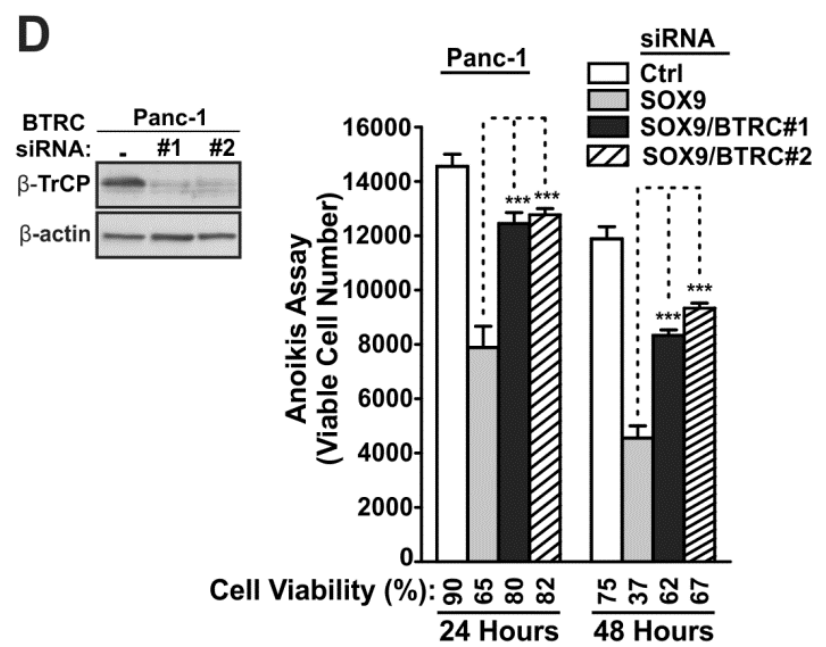

E

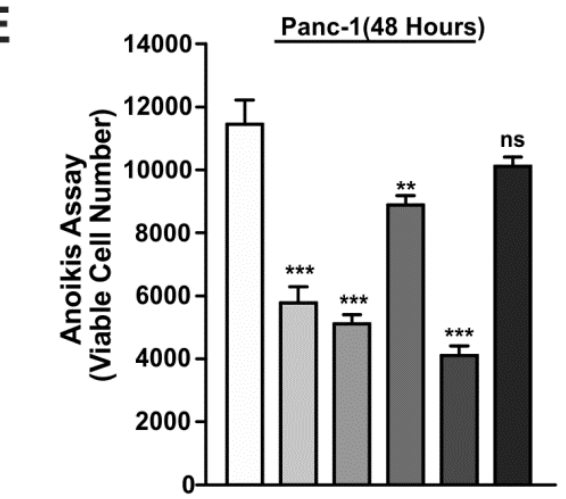

Cell Viability (\%):

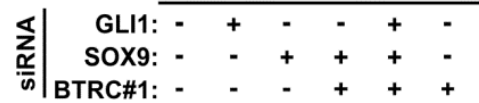

Figure 5. In a GLI1-dependent fashion, $\beta$-TrCP suppression rescues malignant properties in 
SOX9-deficient PDA cells. 
A
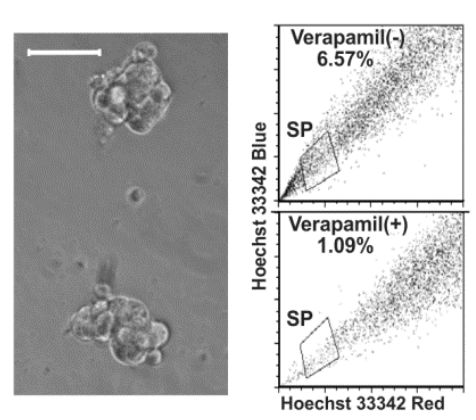

C

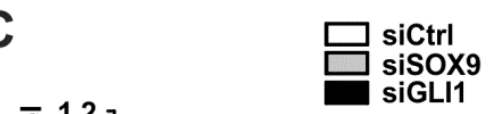

B

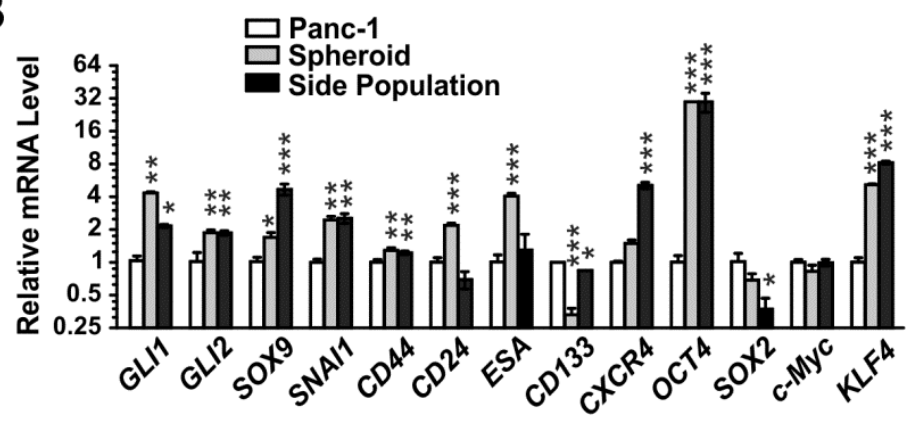

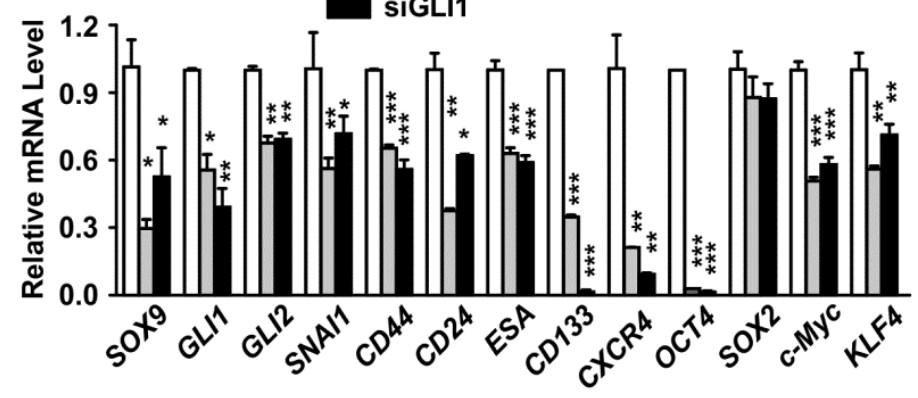
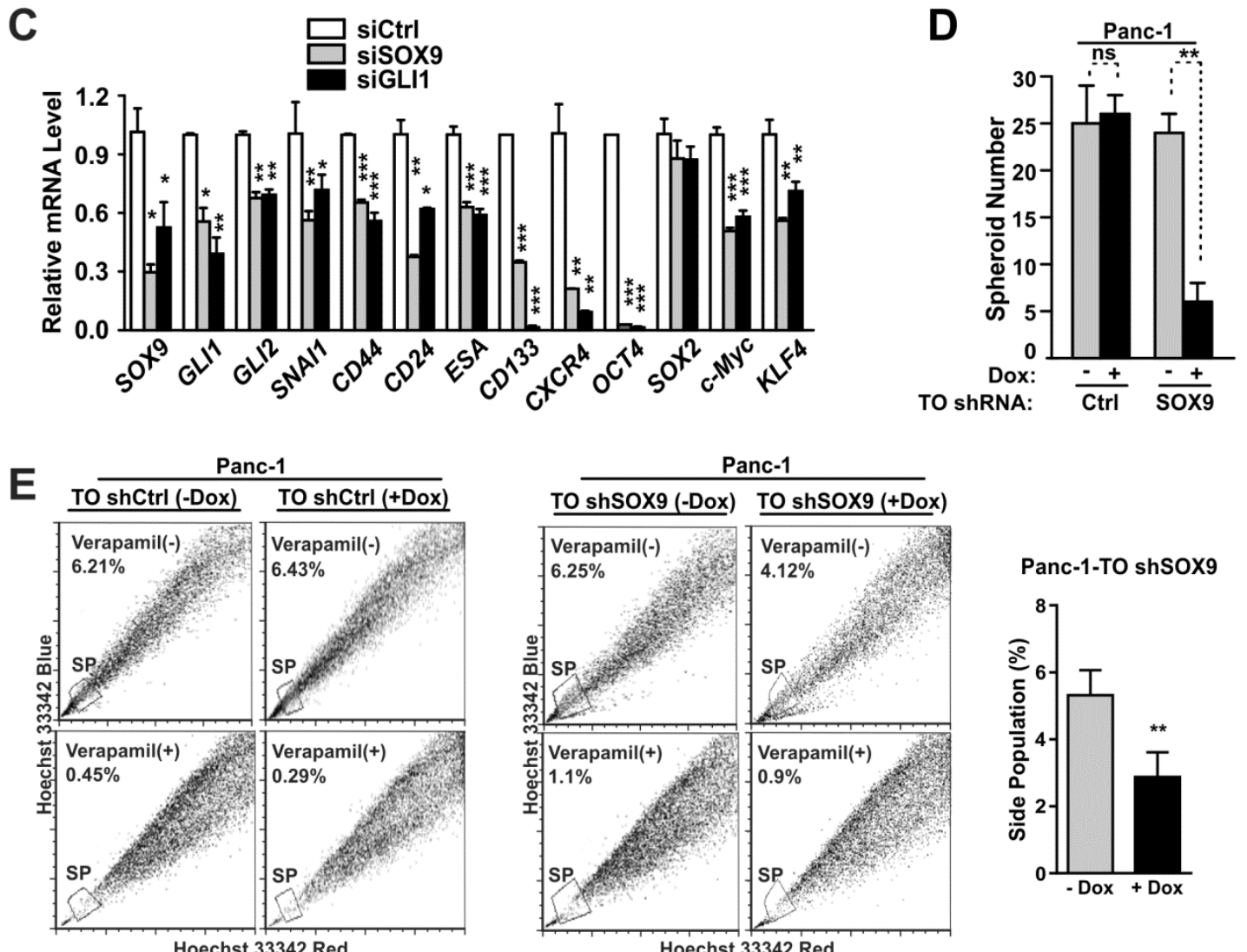

Panc-1

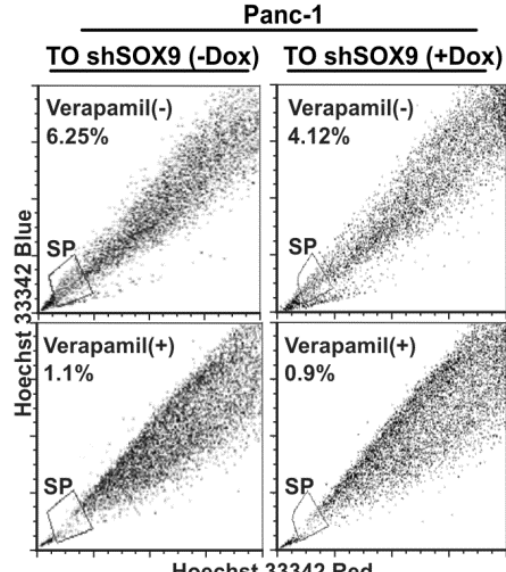

Panc-1-TO shSOX9

$\mathbf{F}$
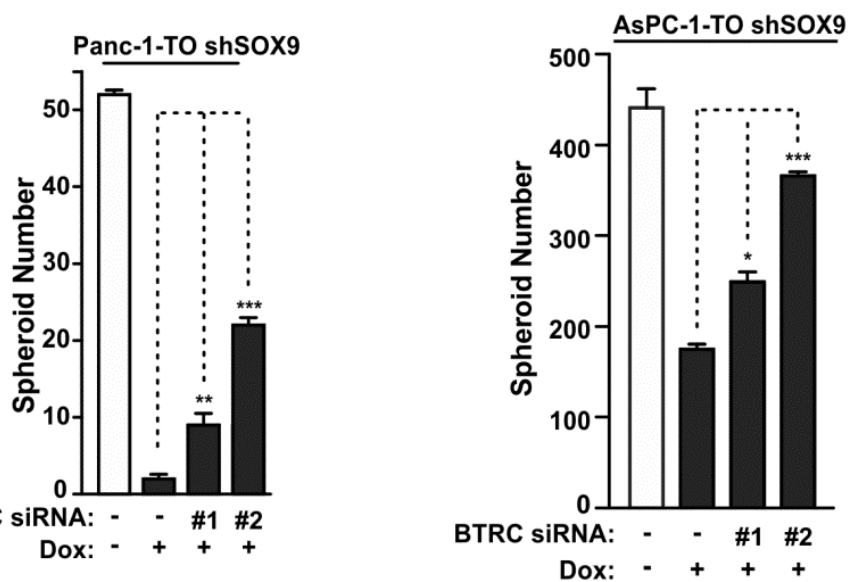

Figure 6. $\beta$-TrCP suppression rescues CSC properties in SOX9-deficient PDA cells. 


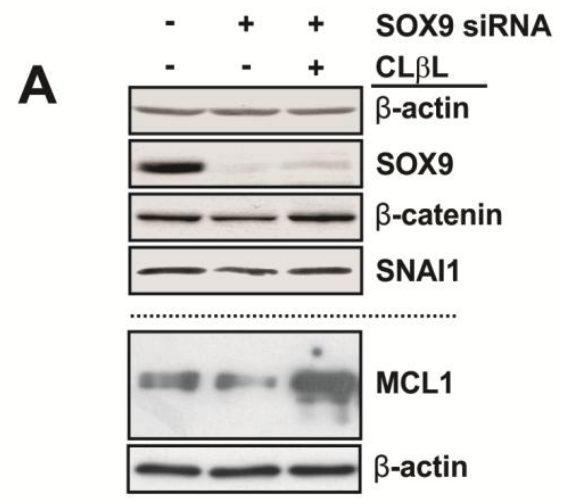

C
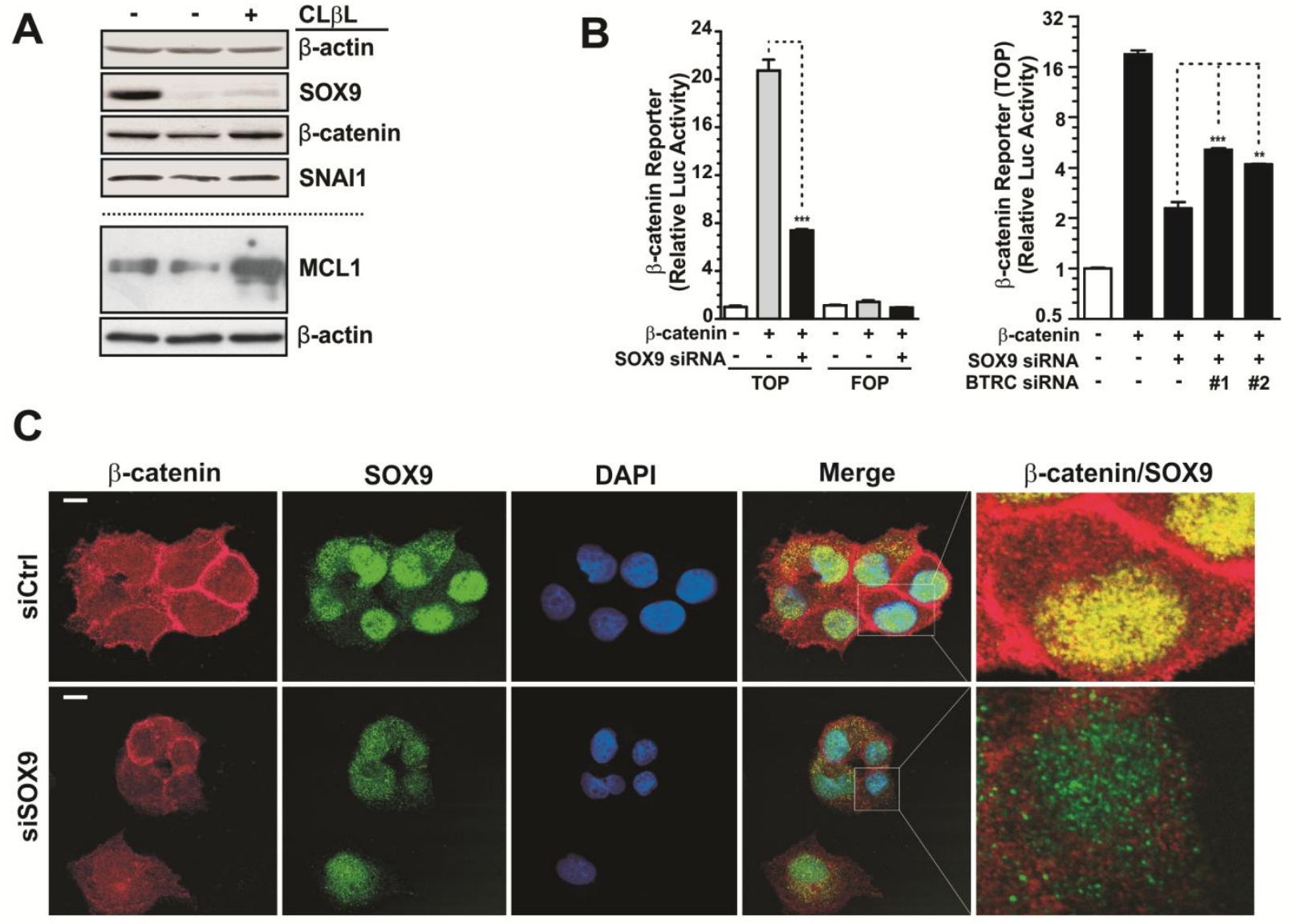

D

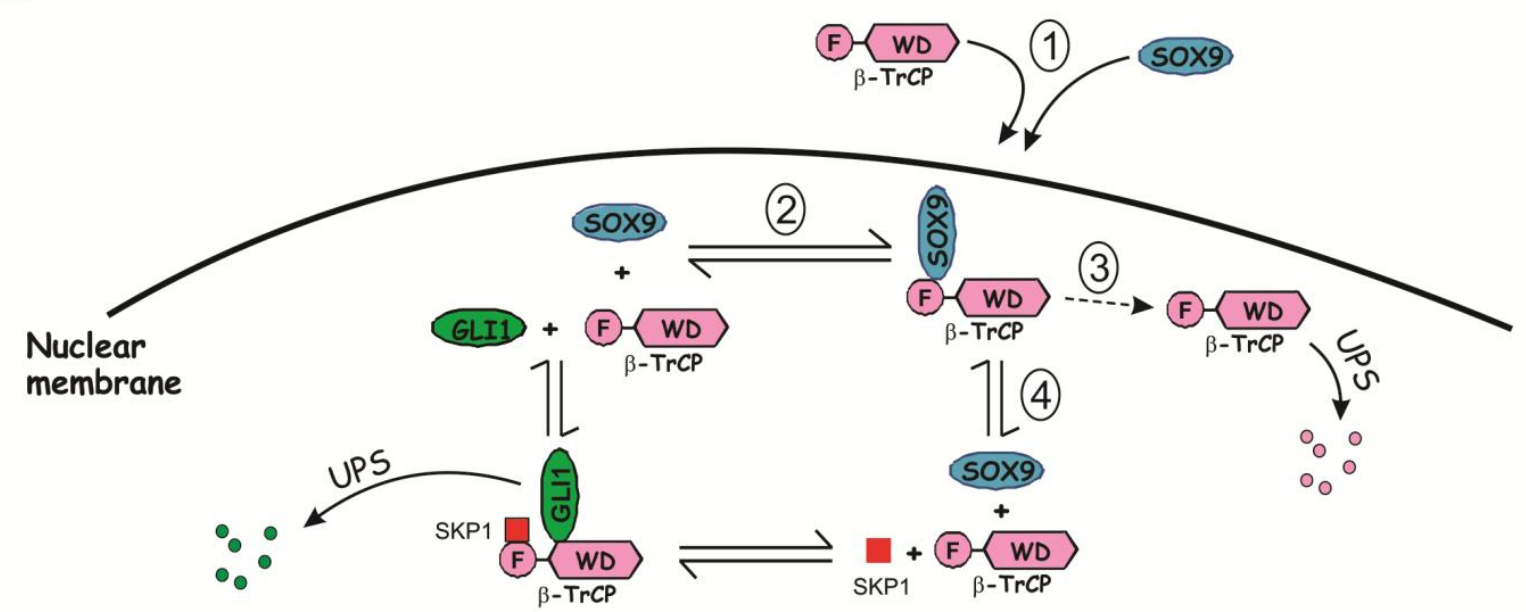

Figure 7. SOX9 stabilizes $\beta$-catenin in Panc- 1 cells. 
Table S1: Microarray expression analyses of Sox 9 and $\beta$-TRCP in primary pancreatic cancer.

\begin{tabular}{|c|c|c|c|c|c|c|c|c|c|}
\hline \multirow{2}{*}{$\begin{array}{l}\text { Data Source } \\
\text { (Reference) }\end{array}$} & \multicolumn{3}{|c|}{ Sox9 (Cancer vs Normal) } & \multicolumn{3}{|c|}{$\beta$-TrCP (Cancer vs Normal) } & \multicolumn{3}{|c|}{ Stratifin (Cancer vs Normal) } \\
\hline & Probe & P value & Fold & Probe & P value & Fold & Probe & P value & Fold \\
\hline \multirow[t]{2}{*}{ Badea et al., 2008} & \multirow[t]{2}{*}{ 202936_s_at } & \multirow[t]{2}{*}{0.0020} & \multirow[t]{2}{*}{1.87} & 204901_at & $6.5 \mathrm{E}-07$ & -1.22 & \multirow[t]{2}{*}{ 33323_r_at } & \multirow[t]{2}{*}{$1.8 \mathrm{E}-11$} & \multirow[t]{2}{*}{6.66} \\
\hline & & & & 222374_at & $1.2 \mathrm{E}-05$ & -1.21 & & & \\
\hline $\begin{array}{c}\text { Iacobuzio-Donahue } \\
\text { et al., } 2003\end{array}$ & $\begin{array}{c}\text { IMAGE:75318 } \\
4\end{array}$ & 0.19 & 1.35 & $\begin{array}{c}\text { IMAGE:4616 } \\
28\end{array}$ & 0.061 & -1.25 & $\begin{array}{c}\text { IMAGE: } 2027 \\
515\end{array}$ & $1.1 \mathrm{E}-06$ & 20.2 \\
\hline Pei et al., 2009 & 202936_s_at & 0.014 & 1.79 & 222374_at & 0.034 & -1.12 & 33323_r_at & $2.3 \mathrm{E}-08$ & 19.0 \\
\hline Segara et al., 2005 & 202936_s_at & 0.50 & 1.00 & 204901_at & 0.026 & -1.23 & 33323_r_at & $3.3 \mathrm{E}-06$ & 9.74 \\
\hline
\end{tabular}


Table S2: Oligonucleotide sequences for siRNA and shRNA

\begin{tabular}{|c|c|l|}
\hline siRNA/shRNA & Target sequence (5'-3') & \multicolumn{1}{c|}{ Source } \\
\hline Ctrl & Firefly luciferase & $\begin{array}{l}\text { siGENOME Non-Targeting siRNA \#2, Thermo } \\
\text { Scientific (Dharmacon), D-001210-02 }\end{array}$ \\
\hline Ctr12 & GATGGGGAGTTTCCAGGTTCA & Thermo Scientific (Dharmacon), custom \\
\hline BTRC \#1 & GCGACATAGTTTACAGAGA & $\begin{array}{l}\text { siGENOME siRNA, Thermo Scientific (Dharmacon), } \\
\text { D-044048-04 }\end{array}$ \\
\hline BTRC \#2 & Smart Pool & $\begin{array}{l}\text { siGENOME SMARTpool siRNA, Thermo Scientific } \\
\text { (Dharmacon), M-003463-01 }\end{array}$ \\
\hline GLI1 & Smart Pool & $\begin{array}{l}\text { ON-TARGETplus SMARTpool siRNA, Thermo } \\
\text { Scientific (Dharmacon), L-003896-00 }\end{array}$ \\
\hline SOX9 & GAACGCACATCAAGACGGA & $\begin{array}{l}\text { siGENOME siRNA, Thermo Scientific (Dharmacon), } \\
\text { D-059108-01 }\end{array}$ \\
\hline TO shCtrl & Scrambled sequence & $\begin{array}{l}\text { Non-silencing TRIPZ lentiviral inducible shRNAmir } \\
\text { control (Open Biosystems, RHS4743) }\end{array}$ \\
\hline TO shSOX9 & ACCCGCTCACAGTACGACT & $\begin{array}{l}\text { The hairpin sequence from pGIPZ-shRNAmir (Open } \\
\text { Biosystems, V3LHS_396212) was inserted into pTRIPZ. }\end{array}$ \\
\hline Rat Sox9 \#1 & AGAACAAGCCACACGTCAA & Thermo Scientific (Dharmacon), custom \\
\hline Rat Sox9 \#2 & CTGCTGAACGAGAGCGAGA & Thermo Scientific (Dharmacon), custom \\
\hline Rat shCtrl & Scrambled sequence & $\begin{array}{l}\text { Hairpin sequence was inserted into the pSilencer 2.1-U6 } \\
\text { neo vector (Life Technologies, AM5764). }\end{array}$ \\
\hline Rat Sox9 sh1 & GAGAGCGAGGAAGATAAAT & $\begin{array}{l}\text { Hairpin sequence was inserted into the pSilencer 2.1-U6 } \\
\text { neo vector (Life Technologies, AM5764). }\end{array}$ \\
\hline Rat Sox9 sh2 & GGAAGATAAATCCCAGTGTG & $\begin{array}{l}\text { Hairpin sequence was inserted into the pSilencer 2.1-U6 } \\
\text { neo vector (Life Technologies, AM5764). }\end{array}$ \\
\hline
\end{tabular}


Table S3: Oligonucleotides for real-time quantitative PCR analysis

\begin{tabular}{|c|l|l|}
\hline Gene & \multicolumn{1}{|c|}{ Sense (5'-3') } & \multicolumn{1}{c|}{ Antisense (5'-3') } \\
\hline$B T R C$ & CTGCAGGGACACTCTGTCTAC & GAAGTCCCAGATGAGGATTGTG \\
\hline$C T N N B 1$ & CCCACTGGCCTCTGATAAAGG & ACGCAAAGGTGCATGATTTG \\
\hline$c-M y c$ & CGACGAGACCTTCATCAAAA & TGCTGTCGTTGAGAGGGTAG \\
\hline$C D 24$ & AAACAACAACTGGAACTTCAAGTAACT & GGTGGTGGCATTAGTTGGATTT \\
\hline$C D 44$ & TGCCGCTTTGCAGGTGTAT & GGCCTCCGTCCGAGAGA \\
\hline$C D 133$ & TGGATGCAGAACTTGACAACGT & ATACCTGCTACGACAGTCGTGGT \\
\hline$C X C R 4$ & GCCTTATCCTGCCTGGTATTGTC & GCGAAGAAAGCCAGGATGAGGAT \\
\hline$E S A$ & GCAGCTCAGGAAGAATGTG & CAGCCAGCTTTGAGCAAATGAC \\
\hline$G A P D H$ & TCACCACCATGGAGAAGGC & GCTAAGCAGTTGGTGGTGCA \\
\hline$G L I 1$ & ACAGAAGGACTGTCTGGCCCGC & GGTGAGATGGACAGTGCCCGC \\
\hline$G L I 2$ & GCCCTTCCTGAAAAGAAGAC & CATTGGAGAAACAGGATTGG \\
\hline$K L F 4$ & AGAGTTCCCATCTCAAGGCA & GTCAGTTCATCTGAGCGGG \\
\hline$O C T 4$ & GAAGCAGAAGAGGATCACCTTG & TTCTTAAGGCTGAGCTGCAAG \\
\hline$S N A I 1$ & TCTGAGTGGGTCTGGAGGTG & CTCTAGGCCCTGGCTGCTAC \\
\hline$S O X 2$ & AACCCCAAGATGCACAACTC & GCTTAGCCTCGTCGATGAAC \\
\hline$S O X 9$ & CCCTTCGTGGAGGAGGCGGA & CCGGAGGAGGAGTGTGGCGA \\
\hline Rat $B c l 2$ & CGGAGGCTGGGATGCCTTTGT & TGTGGCCCAGGTATGCACCCA \\
\hline Rat Gapdh & TCACCACCATGGAGAAGGC & GCTAAGCAGTTGGTGGTGCA \\
\hline Rat $P t c h 1$ & CCAGGCTGCTGTGGTGGTGG & CCGGCTGACACAGGGGCTTG \\
\hline Rat Sox 9 & GCGAGGAAGATAAATTCCCAGTG & GTGGTCTTTCTTGTGCTGCACGC \\
\hline
\end{tabular}




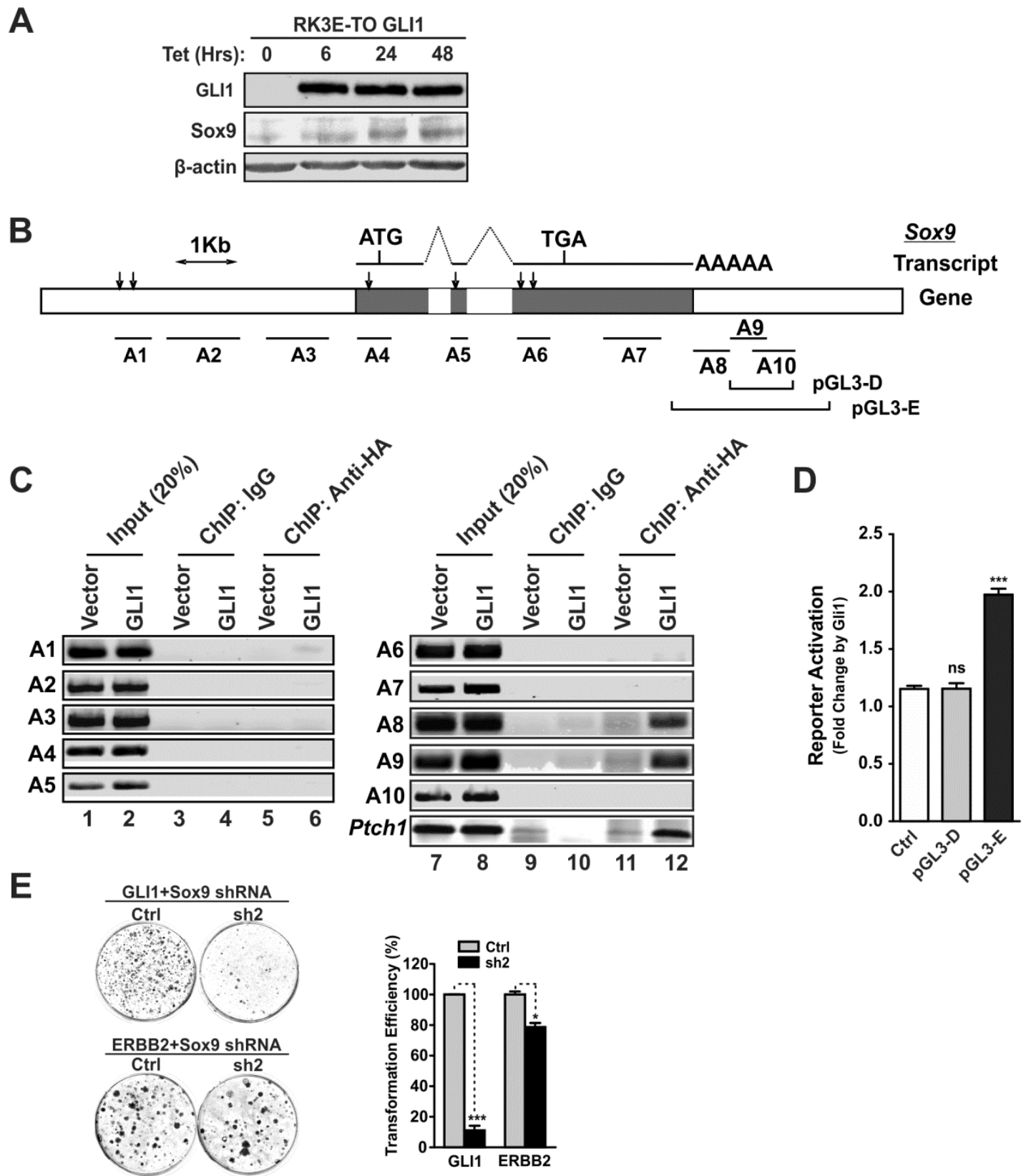

Figure S1. Sox9 is activated by GLI1 in the rat RK3E epithelial model and is required for GLI1mediated malignant transformation. (A) RK3E-derived, tet-on (TO) GLI1 cells were induced with tet and cell extracts were analyzed by immunoblot. (B) Schematic of the RnSox9 locus. Potential GLI-binding sites (arrows) each contain one mismatch to the consensus GACCACCCA. ChIP-analyzed fragments (A1-A10) and fragments inserted into a luciferase reporter (pGL3-D, pGL3-E) are indicated below. Primer sequences are listed in Table S3 in the 
supplemental material. (C) ChIP analysis of Sox9 gene in RK3E cells. Chromatin was prepared from RK3E cells stably-transduced with HA-GLI1 retrovirus (GLI1) or empty vector (Vector). Ptch1 served as a positive control. (D) Regulation of luciferase reporter activity by GLI1 relative to empty vector in HEK293 cells. (E) In vitro transformation by GLI1 or ERBB2 was assessed in RK3E cells. ***, $\mathrm{P}<0.001 ;$ ns, not significant. 

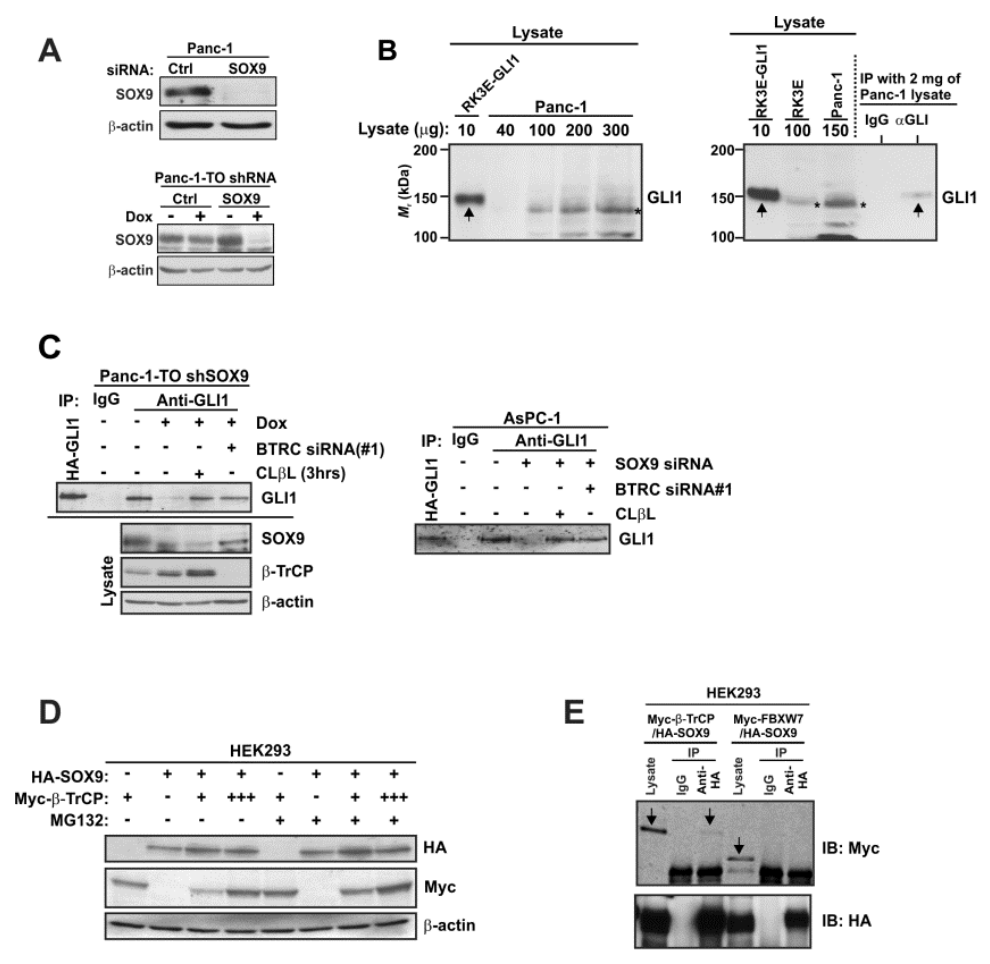

Figure S2. SOX9 interrupts $\beta$-TrCP-mediated GLI1 degradation. (A) SOX9 suppression mediated by transient siRNA or stable, conditional vector (TO-shSOX9) in Panc-1 cells. These two strategies target distinct sequences within SOX9. (B) Analysis of GLI1 protein in Panc-1 cells by immunoblot or IP-immunoblot analysis. Left panel: RK3E cells expressing exogenous GLI1 served as a control. By immunoblot a background species (asterisk) is detected in Panc-1 cells. In contrast, IP-immunoblot detects endogenous GLI1 (right panel). (C) SOX9 stabilizes the GLI1 protein in PDA cells. Left panel: Cells were treated with dox or vehicle control for 5 days and then analyzed by IP-immunoblot analysis. Right panel: Cells were treated as indicated and then analyzed by IP-immunoblot analysis. (D) Despite interacting with b-TrCP, SOX9 expression is not suppressed by the exogenous F-box protein. Instead, SOX9 suppresses b-TrCP expression in a proteasome-dependent fashion. Cells were transfected with the indicated vectors and treated with proteasome inhibitor (MG132) or vehicle (DMSO) prior to immunoblot analysis. The Myc- $\beta$-TrCP plasmid quantity in the transfection mixture was three-fold higher in 
the indicated lanes (+++). (E) SOX9 selectively interacts with b-TrCP as compared to another Fbox protein, FBXW7. Co-IP analysis of HA-SOX9 with Myc-tagged F box proteins in HEK293 cells after transient expression. Lysate lanes represent $5 \%$ of the input extract. 
A

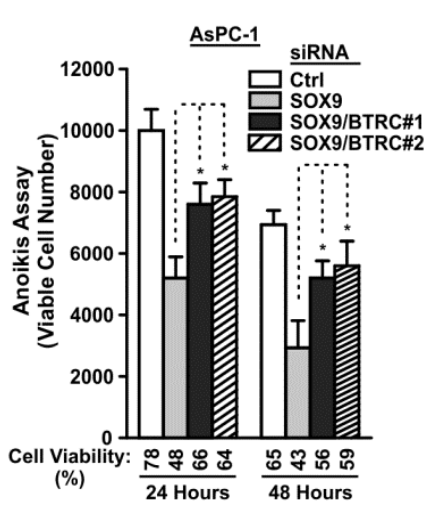

B
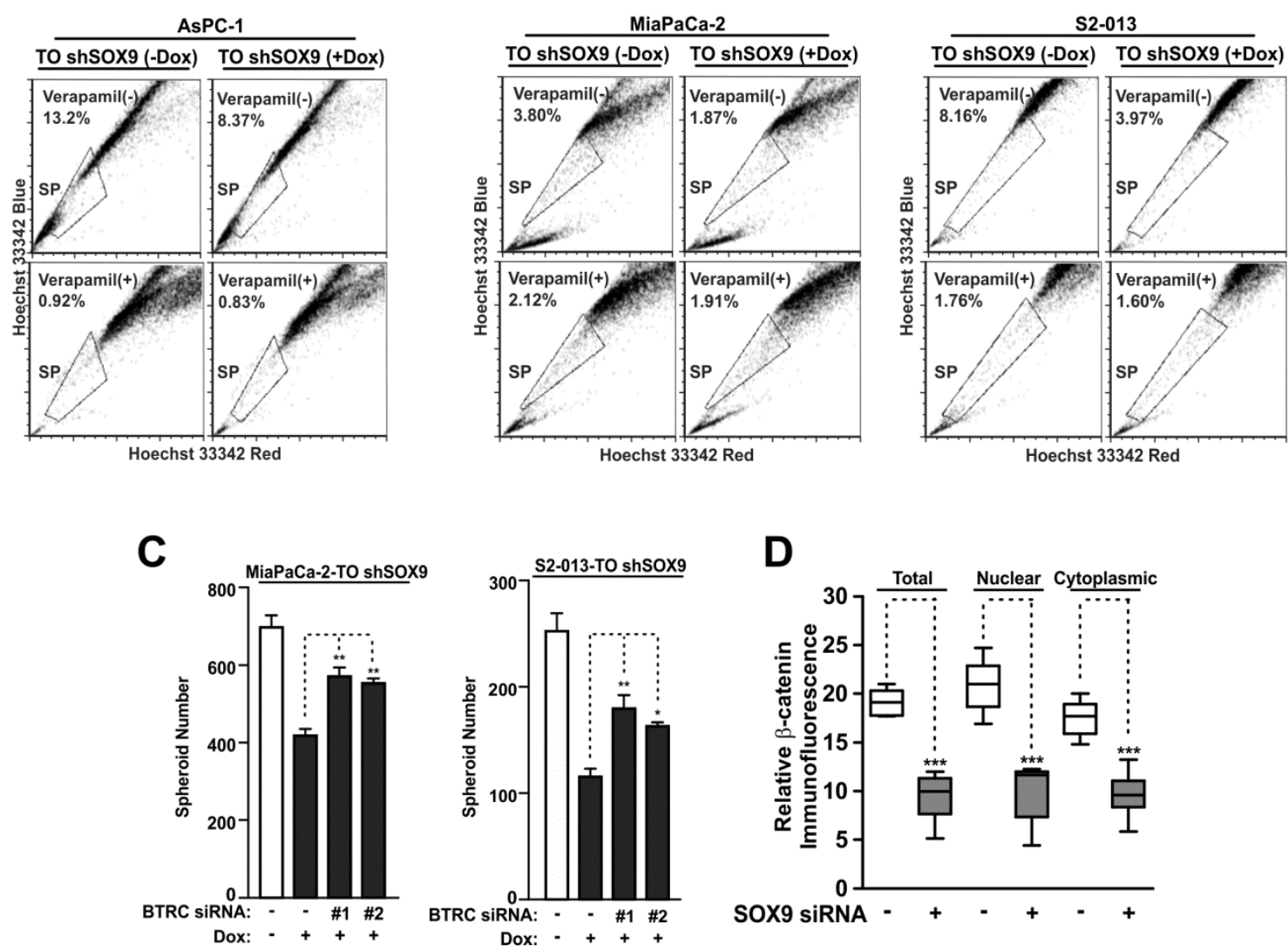

Figure S3. SOX9 is critical for cancer cell malignant properties, cancer stem cell properties and expression of $\beta$-catenin in PDA cells. (A) Anoikis assays were performed in AsPC-1 using two $\beta$-TrCP siRNAs. (B) PDA cells were treated with dox or vehicle for 5 days and then analyzed for Side population (SP) cells. (C) Roles of SOX9 and b-TrCP in tumor spheroid growth. (D) Regulation of b-catenin by SOX9. Panc-1 cells were treated with the indicated siRNA and the expression of $\beta$-cateninin was quantitated in the nucleus and cytoplasm. For each sample, 
confocal images were captured for ten randomly selected fields and immunofluorescence was quantitated using Image $\mathrm{J}$. 


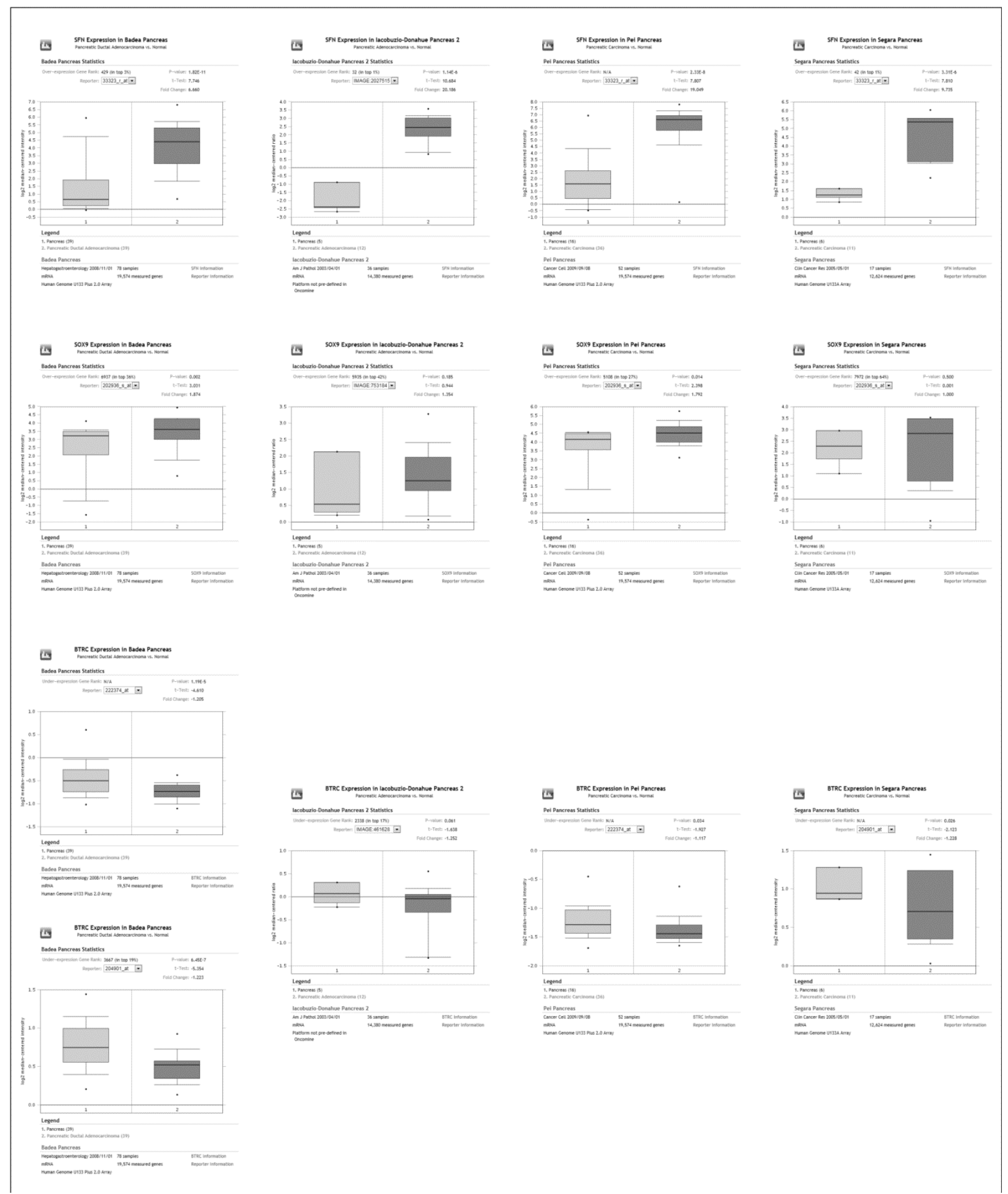

Figure S4. Gene expression profile analysis of human PDA. Human pancreatic carcinoma samples and normal pancreatic tissue were analyzed for expression of SOX9 and BTRC. Published studies that showed upregulation of established PDA markers such as SFN (14-3-3s) 
were selected for analysis of SOX9 and BTRC. Box plots indicate median and inter-quartile range (box), 10th and 90th percentiles (whiskers), and minimum and maximum values (asterisk). 


\section{CHAPTER 3}

\section{KLF4 Suppresses Expression of the Receptor Tyrosine Kinase DDR1 in Breast Cancer}

\section{Cells and Inhibits Spontaneous Metastasis}

Daniel B. Vanderbilt ${ }^{1,2}$, Sriganesh B. Sharma ${ }^{1,2}$, Chen-Chung Lin ${ }^{1,2}$, and J. Michael Ruppert ${ }^{1,2,3^{*}}$

${ }^{1}$ Department of Biochemistry, ${ }^{2}$ Program in Cancer Cell Biology, and ${ }^{3}$ The Mary Babb Randolph Cancer Center, West Virginia University, Morgantown, West Virginia 26506

*Author for correspondence: mruppert@hsc.wvu.edu 


\begin{abstract}
In breast cancer cells, Krüppel-like factor 4 (KLF4) is recognized to suppresses apoptosis and is upregulated in the cancer stem cell population. Still, the role of KLF4 in human breast cancer remains unclear in many respects. We found endogenous KLF4 to potently suppress spontaneous metastasis of breast cancer cells in a mouse model system. The antimetastatic effect of KLF4 in our studies was not explained by a difference in primary tumor growth, yet we observed increased circulating tumor cells in mice with KLF4 knockdown tumors, suggesting KLF4 acts to inhibit metastasis at an early stage. Suppression of KLF4 was associated with increased expression of discoidin domain receptor 1 (DDR1), a receptor tyrosine kinase that binds collagen and is implicated in cancer progression. In agreement with increased DDR1 function, KLF4 knockdown cells exhibited greater adherence to collagen. The function of KLF4 to suppress metastasis of breast cancer cells may then relate to DDR1 expression.
\end{abstract}




\section{Introduction}

Metastatic breast cancer remains an incurable disease for most patients, although survival is improving $(1,2)$. Research into the molecular events that regulate breast cancer metastasis may help inform the development of new therapies or prognostic models (3). To form clinically relevant metastases, tumor cells must successfully progress through a cascade of events to escape the primary tumor, navigate the circulatory system, and then achieve survival and proliferation at a distant site (4). Failure at any point in the process may be sufficient to inhibit metastasis $(5,6)$. Malignant cells that are successful in the early stages of metastasis, gaining access to the vasculature, can be detected as circulating tumor cells (CTCs), and are of prognostic significance in both early and late stage breast cancer (7). Meanwhile, the later steps of survival and proliferation in ectopic sites are increasingly viewed as limiting factors in cancer metastasis $(4,5,8,9)$.

Kruppel-like factor 4 (KLF4) is a zinc finger transcription factor with numerous biological functions, regulating proliferation, differentiation, and apoptosis $(10,11)$. KLF4 is also a crucial mediator of pluripotency (12-14). In cancer, KLF4 has context-dependent functions that may promote or suppress malignancy (15). Downregulation of KLF4 is associated with carcinomas of the colon (16-20), stomach (21-23), esophagus (24), liver (25), and pancreas (26). In contrast, increased expression of KLF4 has been associated with breast cancer (27), although this result was not reproduced by large scale RNA-sequencing of breast tumors (28).

KLF4 is reported to contribute to malignant properties in breast cancer cells through several mechanisms. Through miR-21 and miR-26, which suppress expression of RASA1 and 
SPRED1, KLF4 enhances RAS/ERK signaling in MDA-MB-231 cells, thereby promoting resistance to anoikis (29). KLF4 also acts to maintain the population of cancer stem cells (CSCs) in breast cancer cell lines, and is upregulated in CSCs isolated from primary tumor cells of the murine MMTC-Neu and C3(1)TAg mouse models, as well as in patient-derived xenografts $(30,31)$. Consistent with established anti-apoptotic roles, KLF4 also promotes resistance to HER2-targeted therapy through upregulation of anti-apoptotic factors MCL1 and BCL-XL (32).

Discoidin domain receptors (DDRs) 1 and 2 are receptor tyrosine kinases (RTKs) that interact through extracellular domains with collagen (33). Specimens of many human tumor types show dysregulated DDR expression (34), including breast cancer (35,36). DDRs have been shown to influence cancer-relevant processes including EMT, adhesion, motility, and invasion, and may also promote metastasis (34). DDRs are poorly understood in comparison to many other RTKs, and the mechanisms underlying the apparent dysregulation of DDRs in malignancy remain largely uncharacterized.

In the present study, we report that KLF4 suppresses spontaneous metastasis of breast cancer cells in a mouse model. This effect was consistent using both gain- and loss-of-function approaches, and was independent of primary tumor volume. Loss of KLF4 significantly enhanced the dissemination of cells from the primary tumor, as demonstrated by increased levels of CTCs. KLF4 knockdown cells were characterized by increased expression of DDR1, and had superior adhesion to collagen I. KLF4 may then possibly exert antimetastatic effects through suppression of DDR1. 


\section{Results}

Suppression of KLF4 enhances spontaneous metastasis of breast cancer cells. The effects of KLF4 in models of breast cancer are variable. Overexpression of KLF4 can readily suppress cell proliferation of murine 4T1 cells, and similarly inhibits orthotopic tumor growth in BALB/c mice (37). In contrast, KLF4 gain-of-function using the KLF4-estrogen receptor (ER) fusion protein (38) enhances growth of Py2T tumors in mice receiving tamoxifen (39). We previously reported that depletion of KLF4 could impair tumor initiation of MDA-MB-231 cells in athymic nude mice, yet did not affect the growth kinetics of tumors that did successfully initiate (31).

In NOD/SCID/ $\gamma$ (NSG) mice, MDA-MB-231 cells are highly metastatic to the lungs and liver (40), and we asked whether suppression of KLF4 would affect spontaneous metastasis in this context. We found that stable suppression of KLF4 in MDA-MB-231 cells (Fig. 1A) did not affect orthotopic tumor initiation, as all mice developed primary tumors. Similarly, we did not observe a statistically significant difference in tumor growth between groups (Fig. 1B). To enumerate lung metastasis, we euthanized mice 32 days after injection, and counted the metastases present on fixed specimens. Suppression of KLF4 resulted in greatly increased spontaneous lung metastasis (Fig. 1C and D). In an independent experiment, we also addressed the function of KLF4 in metastasis to the liver. As expected, expression of KLF4 had little effect on primary tumor growth (Fig. 1E), yet we observed significantly greater liver metastases resultant from KLF4 knockdown. 
We additionally examined the role of KLF4 in spontaneous metastasis using M6 cells, a line derived from mouse mammary tumors of the C3(1)TAg transgenic model (41). The shRNAmediated suppression of KLF4 in M6 cells was incomplete (Fig. 2A). Still, the partial loss of KLF4 still favored enhanced metastasis to the lungs, without any significant effects on primary tumor volume (Fig. 2B-D). These data indicate endogenous KLF4 can function to suppress the spontaneous metastasis of breast cancer cells.

\section{Overexpression of KLF4 suppresses spontaneous metastasis of breast cancer cells.}

To date, several reports have suggested that KLF4 overexpression can suppress spontaneous metastasis, including results in breast cancer cells $(37,39)$, and in cell lines representing gastrointestinal malignancies (42-44). Metastasis suppressor proteins, in which metastasis is inhibited without meaningful effects on primary tumor growth, are also largely characterized based on the effects of ectopic expression (45). Although we have previously observed retroviral-mediated overexpression of KLF4 to suppress cell proliferation in culture (46), we wondered whether ectopic KLF4 would exert a similar effect in breast cancer cells in vivo. In NSG mice, the stable overexpression of KLF4 using a retroviral vector did not significantly alter tumor growth of MDA-MB-231 cells (Fig. 3A) or MDA-MB-435 cells (Fig. 3C). In agreement with role of endogenous KLF4 (Fig. 1 and 2), overexpression of KLF4 resulted in decreased liver metastasis of MDA-MB-231 cell (Fig. 3B), and decreased lung metastasis of MDA-MB435 cells (Fig. 3D).

KLF4 suppresses affects metastasis at an early stage. Our data suggest KLF4 is a suppressor of metastasis in breast cancer cells. While this effect was clearly independent of primary tumor growth, the specific metastatic process or processes affected by KLF4 remained 
unclear. To help clarify the effect of KLF4 expression on the metastatic cascade, we sought to determine the effect of KLF4 on circulating tumor cells (CTCs). As in prior experiments, we observed no significant effect of endogenous KLF4 expression on orthotopic tumor growth (Fig. 4A).

While CTCs are often distinguished from leukocytes based on expression of markers such as epithelial cell adhesion molecule (EpCAM) and other factors (47), we instead indentified CTCs based on fluorescence. This technique was facilitated by the high constitutive GFP expression of the MDA-MB-231 cells as conferred by the shRNA vector. At the time mice were euthanized, we detected approximately three-fold more CTCs as a result of KLF4 suppression (Fig. 4B). There were no overt metastases at this stage.

This assessment of CTCs indicated KLF4 acts at an early stage of metastasis to inhibit the dissemination of cells from the primary tumor. Previous reports have indicated endogenous KLF4 can promote motility and invasion of MDA-MB-231 cells $(30,46)$. We attempted to verify this result using two distinct shRNAs, and as expected, KLF4 knockdown cells exhibited decreased migration and invasion (Figs. 4C and D).

KLF4 suppresses DDR1 expression and collagen interaction. In earlier work, we have identified a role of KLF4 (in submission) in the reprogramming of kinase expression as a response to MEK inhibition (48). These efforts had suggested KLF4 can negatively regulate expression of DDR1. In MDA-MB-231 cells, we found increased expression of DDR1 protein and mRNA resultant from suppression of endogenous KLF4 (Fig. 5A and B). Additionally, Ddrl expression was 7-fold higher increased in mammary tumors of C3(1)/TAg Klf4 ${ }^{+/-}$mice as 
compared to Klf4 homozygous controls (Fig. 5C). We then performed chromatin immunoprecipitation (ChIP) to test whether KLF4 could interact with DDRl regulatory elements. Analysis of putative KLF4 response elements upstream of the DDR1 coding sequence indicated enrichment of KLF4 at one of two sites tested (Fig. 5D). Enrichment at this site was abolished by suppression of KLF4 (Fig. 5E). DDR1 binds and is activated by possibly all collagen types, including collagen I (49). The altered DDR1 expression found in KLF4 knockdown cells suggested that KLF4 might regulate collagen binding, which we determined through adhesion assays. Suppression of KLF4 resulted in significantly increased adhesion of MDA-MB-231 cells to collagen I (Fig. 5F). In contrast, KLF4 did not increase adhesion to a fibronectin substrate.

\section{Discussion}

We have shown that KLF4 suppresses spontaneous metastasis of breast cancer cells in a mouse model, through mechanisms independent of primary tumor growth. Regulation of metastasis may occur at multiple levels in the metastatic cascade, and metastasis suppressor proteins have been shown to function at different steps of metastasis (50). To help distinguish the effects of KLF4 between early and later events in metastatic process, we assessed CTCs in mice with primary tumors of comparable size. In some cases a change in CTCs can be discordant with the finding of metastatic cells; for example, overexpression of miR-200c/miR-141 in breast cancer cells results in enhanced lung colonization even though CTCs are decreased (51). In contrast, our data show agreement between CTC levels (Fig. 4) and spontaneous metastasis (Fig. 1 and 2), associating experimental suppression of KLF4 with increased CTCs and greater metastasis to the lung and liver. While these results do not preclude an effect of KLF4 on later 
events in metastasis, it seems likely that KLF4 in some manner restricts access of primary tumor cells to the vasculature.

Overexpression of KLF4 in breast cancer cells was previously reported to suppress orthotopic tumor growth (37), a result consistent with the well-documented antiproliferative function of KLF4 $(52,53)$. In contrast, we did not observe inhibition of tumor growth in association with exogenous KLF4 (Fig. 3). This may reflect differences amongst cell lines, microenvironment effects of the host mouse strain, or may relate to the expression level of KLF4 protein. It is possible that high expression of KLF4 in breast cancer cells may restrict the growth of primary mammary tumors, whereas a lesser amount of KLF4 could suppress metastasis without noticeably affecting orthotopic growth.

DDR expression is upregulated in many carcinomas, although it is not always clear whether this corresponds to increased receptor activation (34). In non-small cell lung cancer, high DDR1 activation was identified through phosphoproteomic methods (54), supported by reports of DDR1 upregulation (55), association with poor prognosis (56), and possible contribution to metastasis (57). In breast cancer, DDR1 expression may be increased (35), or in some cases decreased in association with DDR2 upregulation (36).

The mechanisms that may explain the role of DDRs in breast cancer and other malignancies remain poorly understood. Experimentally, both soluble and immobilized collagens are capable of activating DDRs (58), resulting in DDR autophosphorylation of tyrosine residues to create docking sites for proteins with SH2, SH3 and PTB domains (34). DDR signaling can result in activation of downstream pathways such as RAS/MAPK and NF- $\mathrm{KB}(59,60)$. We have 
previously reported KLF4 suppression in MDA-MB-231 cells to result in decreased RAS/ERK activation (29), suggesting that the increased DDR1 levels in KLF4 knockdown cells (Fig. 5) may promote metastasis through alternative mechanisms.

In a mouse model of metastasis to bone, suppression of DDR1 inhibited the homing and colonization of lung cancer cells (57). Similarly, suppression of DDR2 resulted in decreased metastasis of melanoma cells to the liver (61). Endogenous DDR2 is also reported to promote spontaneous metastasis of breast cancer cells (62). In agreement, our data associate increased expression of DDR1 with spontaneous metastasis of breast cancer cells. It seems likely that the increase in CTCs observed as a result of KLF4 suppression (Fig. 4B), in association with upregulated DDR1 (Fig. 5), is related to an effect on invasion in vivo that is not recapitulated by transwell assay (Fig. 4D). We intend to characterize the functions of KLF4 in breast cancer cells within a 3D collagen matrix. In comparison to $2 \mathrm{D}$ assessments of motility and invasion, a 3D matrix may offer a better recapitulation of the physiologic role of DDR1.

\section{Materials and methods}

Cell lines and cell culture. MDA-MB-231 cells, obtained from Katri S. Selander, and M6 cells, provided by Jeffrey E. Green (National Cancer Institute), were cultured in Dulbecco's modified Eagle's medium (DMEM) containing 10\% fetal bovine serum (FBS) by volume. MDA-MB-435 cells were provided by Danny R. Welch (University of Kansas) and cultured in media containing equal parts DMEM and Ham's F12, with 5\% FBS and 1\% non-essential amino acids solution. 
Viral transduction. Stable suppression of KLF4 was achieved using the following pGIPZ shRNA vectors (GE Dharmacon): In MDA-MB-231 cells, V2LHS_28277 as shKLF4(1) and V3LHS_410934 as shKLF4(2); in M6 cells, V3LMM_459916 as shKLF4(1) and V3LMM_524009 as shKLF4(2). The pBabePuro KLF4 expression vector was previously described (46). Transductions were performed as previously described (46). MDA-MB-231 and M6 lines were selected and maintained using $1 \mu \mathrm{g} / \mathrm{mL}$ puromycin. MDA-MB-435 lines were selected using $3 \mu \mathrm{g} / \mathrm{mL}$ puromycin and maintained in $1 \mu \mathrm{g} / \mathrm{mL}$ puromycin.

C3(1)TAg mammary tumors. C3(1)/TAg Klf4 ${ }^{+/+}$and $C 3(1) / T A g ~ K l f 4^{+/-}$mice, and the harvesting of mammary tumors for analysis by qRT-PCR, were previously described (31).

Immunoblot and PCR, and ChIP. Protein detection by immunoblot was performed as previously described (46). Primary antibodies were used against KLF4 (H180, Santa Cruz), DDR1 (D1G6, Cell Signaling), and $\beta$-actin (C4, Santa Cruz). RNA isolation, reverse transcription, and quantitative real-time PCR using SYBR Green (Life Technologies) were performed as previously described (46). Primers used were human $D D R 1,5^{\prime}-$ CACCAAGAGCAGCAGCAGTA-3' and 5'-GGACTTGAAGGAATGCCAAG-3'; human B2M, 5'-TGCTCGCGCTACTCTCTCTTT-3' and 5'-TCTGCTGGATGACGTGAGTAAAC-3'; mouse Klf4, 5'-GGAAAAGAACAGCCACCCAC-3' and 5'-GTGGTAAGGTTTCTCGCCTGT3'; mouse $D d r 1,5^{\prime}$-TGGATCCCTCTGGTCTATGG-3' and 5'-TCAGACCTGGATCCCCAA3'; mouse Rplp0, 5'-GGACCCGAGAAGACCTCCTT-3' and 5'-

GCACATCACTCAGAATTTCAATGG-3'. ChIP to assess KLF4 binding sites was performed as previously described (29). Putative KLF4 binding sites to DDRI and primers used were ChIP site 1 (-169/+75) (functional): 5'-CTCCTAGATTCTCCCCCTCT-3' and 5'- 
AATGTGACCATGGCAACCAAGT-3'; DDR1 ChIP site 2 (-959/-810) (non-functional): 5'AACTCTCCCTCTCAGACGCA-3' and 5'-ATGATCCCAAAGCCAAAGGT-3'.

Transwell migration and invasion. Migration and invasion assays were performed using a transwell kit as described previously (29). Initial seeding was $1 \times 10^{4}$ cells per well.

Statistics. Tumor growth rates were compared using repeated measures ANOVA with Dunnett's Multiple Comparison test. Other data was compared using ANOVA, with Dunnett posttest to compare against the control condition. Statistical calculations were performed using GraphPad Prism version 5.01. Statistical significance is noted as *, $\mathrm{p}<0.05$; $* *, \mathrm{p}<0.01 ; * * *, \mathrm{p}<0.001$; NS, non-significant.

Animal models and metastasis. All animal procedures and studies were performed under approval of the West Virginia University Animal Care and Use Committee. NOD.Cg-Prkdc scid $I L 2 \mathrm{rg}^{\text {tmlwjl}} / \mathrm{SzJ}$ (NSG) mice were purchased (JAX) or locally bred. Females were used for experiments at 6-10 weeks of age, and experimental groups were age-matched. For injection, cells were cultured to $>80 \%$ confluence and dissociated by trypsin. Each injection consisted of 2 x $10^{6}$ cells suspended in $100 \mu \mathrm{L}$ serum-free DMEM. Injections were performed into the left fourth mammary fat pad. Tumor volume was assessed at least twice per week by calipers, using the formula $\pi / 6 *$ length * width ${ }^{2}$. Mice were euthanized by $\mathrm{CO}_{2}$ asphyxiation. To quantify metastasis, lung and liver specimens were immersed in Bouin's fixative for at least 24 hours, dissected into component lobes, and examined under a dissecting microscope (63). 
CTC analysis. Blood for CTC analysis was drawn by cardiac puncture, using a 25 gauge needle and 3mL syringe prewashed with 500mM EDTA, performed immediately following euthanasia. The blood was then added to an EDTA venous collection tube (BD). RBC lysis by ammonium chloride, and resuspension of the remaining cellular fraction, were performed as previously described (31). Specimens were analyzed using a BD LSRFortessa, with CTC/mL calculated as the number of GFP+ cells divided by the blood specimen volume. The efficacy of this method was first verified using blood drawn from tumor-free mice and inoculated with known quantities of GFP-expressing tumor cells.

Adhesion assays. In a 96 well low-attachment plate, wells were coated with $5 \mu \mathrm{g} / \mathrm{mL}$ collagen I (BD) or $10 \mu \mathrm{g} / \mathrm{mL}$ fibronectin-like polymer (Sigma), then blocked with $1 \%$ culture-suitable BSA in Hank's balanced salt solution. Non-enzymatic Cell Dissociation Solution (Life Technologies) was used to harvest cultured cells, which were added in the amount of $5 \times 10^{4}$ cells per well in $100 \mu \mathrm{L}$ complete DMEM. Cells were allowed to adhere 30 minutes at 37 degrees Celsius. To remove non-adherent cells, wells were washed $4 \mathrm{x}$ with phosphate-buffered saline (PBS). Remaining cells were quantified by adding $100 \mu \mathrm{L}$ complete DMEM and using the ATPlite kit (PerkinElmer) according to manufacturer protocol. To determine the fraction of adherent cells, this result was divided by the signal obtained from $5 \times 10^{4}$ cells per well adherent in a standard 96-well cell culture plate.

\section{Acknowledgements}

We thank Katri S. Selander, Jeffrey Green, and Danny Welch for providing cell lines. This work was supported by grant NCI RO1 CA127405, by the Mary Babb Randolph Cancer Center, and 
by the Jo and Ben Statler Chair in Breast Cancer Research at West Virginia University. Flow Cytometry experiments were performed in the West Virginia University Flow Cytometry Core Facility, which is supported by the National Institutes of Health equipment grant number S10OD016165 and the Institutional Development Award (IDeA) from the National Institute of General Medical Sciences of the National Institutes of Health under grant numbers P30GM103488 (CoBRE) and P20GM103434 (INBRE). Orthotopic tumor cell injections were performed in the West Virginia University Animal Models \& Imaging Facility, which has been supported by the Mary Babb Randolph Cancer Center and NIH grants P20 RR016440, P30 RR032138/GM103488, and S10 RR026378. 


\section{References}

1. Chia, S. K., C. H. Speers, Y. D'yachkova, A. Kang, S. Malfair-Taylor, J. Barnett, A. Coldman, K. A. Gelmon, S. E. O'reilly, and I. A. Olivotto. 2007. The impact of new chemotherapeutic and hormone agents on survival in a population-based cohort of women with metastatic breast cancer. Cancer 110:973-979.

2. Gennari, A., P. Conte, R. Rosso, C. Orlandini, and P. Bruzzi. 2005. Survival of metastatic breast carcinoma patients over a 20-year period: a retrospective analysis based on individual patient data from six consecutive studies. Cancer 104:1742-1750.

3. Chiang, A. C. and J. Massague. 2008. Molecular basis of metastasis. N Engl J Med 359:2814-2823.

4. Vanharanta, S. and J. Massague. 2013. Origins of metastatic traits. Cancer Cell 24:410-421.

5. Fidler, I. J. 2003. The pathogenesis of cancer metastasis: the 'seed and soil' hypothesis revisited. Nat Rev Cancer 3:453-458.

6. Eccles, S. A. and D. R. Welch. 2007. Metastasis: recent discoveries and novel treatment strategies. Lancet 369:1742-1757.

7. Zhang, L., S. Riethdorf, G. Wu, T. Wang, K. Yang, G. Peng, J. Liu, and K. Pantel. 2012. Meta-analysis of the prognostic value of circulating tumor cells in breast cancer. Clin Cancer Res 18:5701-5710.

8. Chambers, A. F., A. C. Groom, and I. C. MacDonald. 2002. Dissemination and growth of cancer cells in metastatic sites. Nat Rev Cancer 2:563-572.

9. Nguyen, D. X., P. D. Bos, and J. Massague. 2009. Metastasis: from dissemination to organspecific colonization. Nat Rev Cancer 9:274-284.

10. McConnell, B. B., A. M. Ghaleb, M. O. Nandan, and V. W. Yang. 2007. The diverse functions of Kruppel-like factors 4 and 5 in epithelial biology and pathobiology. Bioessays 29:549-557. 
11. McConnell, B. B. and V. W. Yang. 2010. Mammalian Kruppel-like factors in health and diseases. Physiol Rev 90:1337-1381.

12. Takahashi, K. and S. Yamanaka. 2006. Induction of pluripotent stem cells from mouse embryonic and adult fibroblast cultures by defined factors. Cell 126:663-676.

13. Kim, J., J. Chu, X. Shen, J. Wang, and S. H. Orkin. 2008. An extended transcriptional network for pluripotency of embryonic stem cells. Cell 132:1049-1061.

14. Jiang, J., Y. S. Chan, Y. H. Loh, J. Cai, G. Q. Tong, C. A. Lim, P. Robson, S. Zhong, and H. H. Ng. 2008. A core Klf circuitry regulates self-renewal of embryonic stem cells. Nat Cell Biol 10:353-360.

15. Rowland, B. D., R. Bernards, and D. S. Peeper. 2005. The KLF4 tumour suppressor is a transcriptional repressor of p53 that acts as a context-dependent oncogene. Nat Cell Biol 7:1074-1082.

16. Shie, J. L., Z. Y. Chen, M. J. O'Brien, R. G. Pestell, M. E. Lee, and C. C. Tseng. 2000. Role of gut-enriched Kruppel-like factor in colonic cell growth and differentiation. Am J Physiol Gastrointest Liver Physiol 279:G806-G814.

17. Zhao, W., I. M. Hisamuddin, M. O. Nandan, B. A. Babbin, N. E. Lamb, and V. W. Yang. 2004. Identification of Kruppel-like factor 4 as a potential tumor suppressor gene in colorectal cancer. Oncogene 23:395-402.

18. Choi, B. J., Y. G. Cho, J. W. Song, C. J. Kim, S. Y. Kim, S. W. Nam, N. J. Yoo, J. Y. Lee, and W. S. Park. 2006. Altered expression of the KLF4 in colorectal cancers. Pathol Res Pract 202:585-589.

19. Patel, N. V., A. M. Ghaleb, M. O. Nandan, and V. W. Yang. 2010. Expression of the tumor suppressor Kruppel-like factor 4 as a prognostic predictor for colon cancer. Cancer Epidemiol Biomarkers Prev 19:2631-2638.

20. Lee, H. Y., J. B. Ahn, S. Y. Rha, H. C. Chung, K. H. Park, T. S. Kim, N. K. Kim, and S. J. Shin. 2014. High KLF4 level in normal tissue predicts poor survival in colorectal cancer patients. World J Surg Oncol 12:232. 
21. Wei, D., W. Gong, M. Kanai, C. Schlunk, L. Wang, J. C. Yao, T. T. Wu, S. Huang, and K. Xie. 2005. Drastic down-regulation of Kruppel-like factor 4 expression is critical in human gastric cancer development and progression. Cancer Res 65:2746-2754.

22. Katz, J. P., N. Perreault, B. G. Goldstein, L. Actman, S. R. McNally, D. G. Silberg, E. E. Furth, and K. H. Kaestner. 2005. Loss of Klf4 in mice causes altered proliferation and differentiation and precancerous changes in the adult stomach. Gastroenterology 128:935945.

23. Zhang, N., J. Zhang, Z. W. Wang, L. Zha, and Z. Huang. 2012. Altered expression of Kruppel-like factor 4 and beta-catenin in human gastric cancer. Oncol Lett 3:1017-1022.

24. Ma, M. Q., H. D. Zhang, P. Tang, H. J. Jiang, and C. G. Chen. 2014. Association of Kruppel-like factor 4 expression with the prognosis of esophageal squamous cell carcinoma patients. Int J Clin Exp Pathol 7:6679-6685.

25. Lin, Z. S., H. C. Chu, Y. C. Yen, B. C. Lewis, and Y. W. Chen. 2012. Kruppel-like factor 4, a tumor suppressor in hepatocellular carcinoma cells reverts epithelial mesenchymal transition by suppressing slug expression. PLoS One 7:e43593.

26. Zammarchi, F., M. Morelli, M. Menicagli, C. C. Di, K. Zavaglia, A. Paolucci, D. Campani, P. Aretini, U. Boggi, F. Mosca, A. Cavazzana, L. Cartegni, G. Bevilacqua, and C. M. Mazzanti. 2011. KLF4 is a novel candidate tumor suppressor gene in pancreatic ductal carcinoma. Am J Pathol 178:361-372.

27. Foster, K. W., A. R. Frost, P. Kie-Bell, C. Y. Lin, J. A. Engler, W. E. Grizzle, and J. M. Ruppert. 2000. Increase of GKLF messenger RNA and protein expression during progression of breast cancer. Cancer Res 60:6488-6495.

28. Cancer Genome Atlas Network. 2012. Comprehensive molecular portraits of human breast tumours. Nature 490:61-70.

29. Sharma, S. B., C. C. Lin, M. K. Farrugia, S. L. McLaughlin, E. J. Ellis, K. M. Brundage, M. A. Salkeni, and J. M. Ruppert. 2014. MicroRNAs 206 and 21 cooperate to promote RAS-extracellular signal-regulated kinase signaling by suppressing the translation of RASA1 and SPRED1. Mol Cell Biol 34:4143-4164.

30. Yu, F., J. Li, H. Chen, J. Fu, S. Ray, S. Huang, H. Zheng, and W. Ai. 2011. Kruppel-like factor 4 (KLF4) is required for maintenance of breast cancer stem cells and for cell migration and invasion. Oncogene 30:2161-2172. 
31. Lin, C. C., S. B. Sharma, M. K. Farrugia, S. L. McLaughlin, R. J. Ice, Y. V. Loskutov, E. N. Pugacheva, K. M. Brundage, D. Chen, and J. M. Ruppert. 2015. Kruppel-like factor 4 signals through microRNA-206 to promote tumor initiation and cell survival. Oncogenesis 4:e155.

32. Farrugia, M. K., S. B. Sharma, C. C. Lin, S. L. McLaughlin, D. B. Vanderbilt, A. G. Ammer, M. A. Salkeni, P. Stoilov, Y. M. Agazie, C. J. Creighton, and J. M. Ruppert. 2015. Regulation of anti-apoptotic signaling by Kruppel-like factors 4 and 5 mediates lapatinib resistance in breast cancer. Cell Death Dis 6:e1699.

33. Vogel, W. 1999. Discoidin domain receptors: structural relations and functional implications. FASEB J 13 Suppl:S77-S82.

34. Valiathan, R. R., M. Marco, B. Leitinger, C. G. Kleer, and R. Fridman. 2012. Discoidin domain receptor tyrosine kinases: new players in cancer progression. Cancer Metastasis Rev 31:295-321.

35. Barker, K. T., J. E. Martindale, P. J. Mitchell, T. Kamalati, M. J. Page, D. J. Phippard, T. C. Dale, B. A. Gusterson, and M. R. Crompton. 1995. Expression patterns of the novel receptor-like tyrosine kinase, DDR, in human breast tumours. Oncogene 10:569-575.

36. Toy, K. A., R. R. Valiathan, F. Nunez, K. M. Kidwell, M. E. Gonzalez, R. Fridman, and C. G. Kleer. 2015. Tyrosine kinase discoidin domain receptors DDR1 and DDR2 are coordinately deregulated in triple-negative breast cancer. Breast Cancer Res Treat 150:918.

37. Yori, J. L., D. D. Seachrist, E. Johnson, K. L. Lozada, F. W. bdul-Karim, L. A. Chodosh, W. P. Schiemann, and R. A. Keri. 2011. Kruppel-like factor 4 inhibits tumorigenic progression and metastasis in a mouse model of breast cancer. Neoplasia 13:601-610.

38. Foster, K. W., Z. Liu, C. D. Nail, X. Li, T. J. Fitzgerald, S. K. Bailey, A. R. Frost, I. D. Louro, T. M. Townes, A. J. Paterson, J. E. Kudlow, S. M. Lobo-Ruppert, and J. M. Ruppert. 2005. Induction of KLF4 in basal keratinocytes blocks the proliferationdifferentiation switch and initiates squamous epithelial dysplasia. Oncogene 24:1491-1500.

39. Tiwari, N., N. Meyer-Schaller, P. Arnold, H. Antoniadis, M. Pachkov, N. E. van, and G. Christofori. 2013. Klf4 is a transcriptional regulator of genes critical for EMT, including Jnk1 (Mapk8). PLoS One 8:e57329. 
40. Iorns, E., K. Drews-Elger, T. M. Ward, S. Dean, J. Clarke, D. Berry, A. D. El, and M. Lippman. 2012. A new mouse model for the study of human breast cancer metastasis. PLoS One 7:e47995.

41. Holzer, R. G., C. MacDougall, G. Cortright, K. Atwood, J. E. Green, and C. L. Jorcyk. 2003. Development and characterization of a progressive series of mammary adenocarcinoma cell lines derived from the C3(1)/SV40 Large T-antigen transgenic mouse model. Breast Cancer Res Treat 77:65-76.

42. Wei, D., M. Kanai, Z. Jia, X. Le, and K. Xie. 2008. Kruppel-like factor 4 induces p27Kip1 expression in and suppresses the growth and metastasis of human pancreatic cancer cells. Cancer Res 68:4631-4639.

43. Chen, H. Y., Y. M. Lin, H. C. Chung, Y. D. Lang, C. J. Lin, J. Huang, W. C. Wang, F. M. Lin, Z. Chen, H. D. Huang, J. Y. Shyy, J. T. Liang, and R. H. Chen. 2012. miR-103/107 promote metastasis of colorectal cancer by targeting the metastasis suppressors DAPK and KLF4. Cancer Res 72:3631-3641.

44. Li, Q., Y. Gao, Z. Jia, L. Mishra, K. Guo, Z. Li, X. Le, D. Wei, S. Huang, and K. Xie. 2012. Dysregulated Kruppel-like factor 4 and vitamin D receptor signaling contribute to progression of hepatocellular carcinoma. Gastroenterology 143:799-810.

45. Rinker-Schaeffer, C. W., J. P. O'Keefe, D. R. Welch, and D. Theodorescu. 2006. Metastasis suppressor proteins: discovery, molecular mechanisms, and clinical application. Clin Cancer Res 12:3882-3889.

46. Lin, C. C., L. Z. Liu, J. B. Addison, W. F. Wonderlin, A. V. Ivanov, and J. M. Ruppert. 2011. A KLF4-miRNA-206 autoregulatory feedback loop can promote or inhibit protein translation depending upon cell context. Mol Cell Biol 31:2513-2527.

47. Krebs, M. G., R. L. Metcalf, L. Carter, G. Brady, F. H. Blackhall, and C. Dive. 2014. Molecular analysis of circulating tumour cells-biology and biomarkers. Nat Rev Clin Oncol 11:129-144.

48. Duncan, J. S., M. C. Whittle, K. Nakamura, A. N. Abell, A. A. Midland, J. S. Zawistowski, N. L. Johnson, D. A. Granger, N. V. Jordan, D. B. Darr, J. Usary, P. F. Kuan, D. M. Smalley, B. Major, X. He, K. A. Hoadley, B. Zhou, N. E. Sharpless, C. M. Perou, W. Y. Kim, S. M. Gomez, X. Chen, J. Jin, S. V. Frye, H. S. Earp, L. M. Graves, and G. L. Johnson. 2012. Dynamic reprogramming of the kinome in response to targeted MEK inhibition in triple-negative breast cancer. Cell 149:307-321. 
49. Vogel, W. F., R. Abdulhussein, and C. E. Ford. 2006. Sensing extracellular matrix: an update on discoidin domain receptor function. Cell Signal 18:1108-1116.

50. Bohl, C. R., S. Harihar, W. L. Denning, R. Sharma, and D. R. Welch. 2014. Metastasis suppressors in breast cancers: mechanistic insights and clinical potential. J Mol Med (Berl) 92:13-30.

51. Korpal, M., B. J. Ell, F. M. Buffa, T. Ibrahim, M. A. Blanco, T. Celia-Terrassa, L. Mercatali, Z. Khan, H. Goodarzi, Y. Hua, Y. Wei, G. Hu, B. A. Garcia, J. Ragoussis, D. Amadori, A. L. Harris, and Y. Kang. 2011. Direct targeting of Sec23a by miR-200s influences cancer cell secretome and promotes metastatic colonization. Nat Med 17:11011108.

52. Chen, X., E. M. Whitney, S. Y. Gao, and V. W. Yang. 2003. Transcriptional profiling of Kruppel-like factor 4 reveals a function in cell cycle regulation and epithelial differentiation. J Mol Biol 326:665-677.

53. Chen, X., D. C. Johns, D. E. Geiman, E. Marban, D. T. Dang, G. Hamlin, R. Sun, and V. W. Yang. 2001. Kruppel-like factor 4 (gut-enriched Kruppel-like factor) inhibits cell proliferation by blocking G1/S progression of the cell cycle. J Biol Chem 276:3042330428.

54. Rikova, K., A. Guo, Q. Zeng, A. Possemato, J. Yu, H. Haack, J. Nardone, K. Lee, C. Reeves, Y. Li, Y. Hu, Z. Tan, M. Stokes, L. Sullivan, J. Mitchell, R. Wetzel, J. Macneill, J. M. Ren, J. Yuan, C. E. Bakalarski, J. Villen, J. M. Kornhauser, B. Smith, D. Li, X. Zhou, S. P. Gygi, T. L. Gu, R. D. Polakiewicz, J. Rush, and M. J. Comb. 2007. Global survey of phosphotyrosine signaling identifies oncogenic kinases in lung cancer. Cell 131:1190-1203.

55. Ford, C. E., S. K. Lau, C. Q. Zhu, T. Andersson, M. S. Tsao, and W. F. Vogel. 2007. Expression and mutation analysis of the discoidin domain receptors 1 and 2 in non-small cell lung carcinoma. Br. J Cancer 96:808-814.

56. Yang, S. H., H. A. Baek, H. J. Lee, H. S. Park, K. Y. Jang, M. J. Kang, D. G. Lee, Y. C. Lee, W. S. Moon, and M. J. Chung. 2010. Discoidin domain receptor 1 is associated with poor prognosis of non-small cell lung carcinomas. Oncol Rep 24:311-319.

57. Valencia, K., C. Ormazabal, C. Zandueta, D. Luis-Ravelo, I. Anton, M. J. Pajares, J. Agorreta, L. M. Montuenga, S. Martinez-Canarias, B. Leitinger, and F. Lecanda. 2012. Inhibition of collagen receptor discoidin domain receptor-1 (DDR1) reduces cell survival, homing, and colonization in lung cancer bone metastasis. Clin Cancer Res 18:969-980. 
58. Vogel, W., G. D. Gish, F. Alves, and T. Pawson. 1997. The discoidin domain receptor tyrosine kinases are activated by collagen. Mol Cell 1:13-23.

59. Ongusaha, P. P., J. I. Kim, L. Fang, T. W. Wong, G. D. Yancopoulos, S. A. Aaronson, and S. W. Lee. 2003. p53 induction and activation of DDR1 kinase counteract p53-mediated apoptosis and influence p53 regulation through a positive feedback loop. EMBO J 22:12891301.

60. Das, S., P. P. Ongusaha, Y. S. Yang, J. M. Park, S. A. Aaronson, and S. W. Lee. 2006. Discoidin domain receptor 1 receptor tyrosine kinase induces cyclooxygenase- 2 and promotes chemoresistance through nuclear factor-kappaB pathway activation. Cancer Res 66:8123-8130.

61. Badiola, I., P. Villace, I. Basaldua, and E. Olaso. 2011. Downregulation of discoidin domain receptor 2 in A375 human melanoma cells reduces its experimental liver metastasis ability. Oncol Rep 26:971-978.

62. Ren, T., W. Zhang, X. Liu, H. Zhao, J. Zhang, J. Zhang, X. Li, Y. Zhang, X. Bu, M. Shi, L. Yao, and J. Su. 2014. Discoidin domain receptor 2 (DDR2) promotes breast cancer cell metastasis and the mechanism implicates epithelial-mesenchymal transition programme under hypoxia. J Pathol 234:526-537.

63. Welch, D. R. 1997. Technical considerations for studying cancer metastasis in vivo. Clin Exp Metastasis 15:272-306. 
FIG. 1. Suppression of KLF4 enhances spontaneous metastasis of MDA-MB-231 cells to the lungs and liver. (A) Immunoblot of KLF4 levels in MDA-MB-231 cells stably expressing shRNA targeting KLF4 or scrambled control. (B-D) Effect of KLF4 expression on spontaneous lung metastasis. (B) Volume of orthotopic tumors in NSG mice (error bars represent SEM). (C) Intact lung specimens, after fixation in Bouin's solution (upper panels), with magnified view of metastatic foci (lower panels). (D) Quantification of lung surface metastases. Each point represents the total lung surface metastases from an individual mouse, obtained from the sum of metastases present on each component lobe of the lungs (bars, SEM). (E-G) Effect of KLF4 expression on spontaneous liver metastasis. (E) Volume of orthotopic MDA-MB-231 tumors in NSG mice (error bars, SEM). (F) Intact liver specimens, after fixation in Bouin's solution. (G) Quantification of liver surface metastases. Each point represents the total liver surface metastases from an individual mouse, obtained from the sum of metastases present on each component lobe of the liver (bars, SEM).

FIG. 2. Suppression of KLF4 enhances spontaneous metastasis of M6 cells to the lungs. (A) Immunoblot of KLF4 levels in M6 cells stably expressing shRNA targeting KLF4 or scrambled control. (B-D) Effect of KLF4 expression on spontaneous lung metastasis. (B) Volume of orthotopic tumors in NSG mice (bars represent SEM). (C) Intact lung specimens, after fixation in Bouin's solution. (D) Quantification of lung surface metastases (bars, SEM).

FIG. 3. Overexpression of KLF4 suppresses spontaneous metastasis of MDA-MB-231 and MDA-MB-435 cells. (A) Immunoblot of KLF4 protein in MDA-MB-231 cells stably expressing exogenous KLF4 or vector control. (B) Volume of orthotopic tumors in NSG mice (bars, SEM). (C) Quantification of liver surface metastases (bars, SEM). (D) Immunoblot of KLF4 levels in 
MDA-MB-435 cells with stably expressing exogenous KLF4 or vector control. (E) Volume of orthotopic tumors in NSG mice (bars, SEM). (F) Quantification of lung surface metastases (error bars, SEM).

FIG. 4. Effect of KLF4 expression on CTC levels, migration, and invasion of MDA-MB-231 cells. Cell lines are from Fig. 1A. (A) Volume of orthotopic tumors in NSG mice (bars, SEM). (B) Concentration of circulating tumor cells expressed as CTCs per mL of blood (bars, SEM). (C-D) Cell migration (C) and invasion (D) as determined by a Boyden chamber transwell assay (bars, SD).

FIG. 5. KLF4 suppresses DDR1 expression. (A) Immunoblot of indicated proteins in MDAMB-231 cells stably expressing shRNA targeting KLF4 or scrambled control. ERK2 is used as a loading control. (B) Expression of DDR1 mRNA in MDA-MB-231 cells by qRT-PCR (bars, SEM). Reactions were normalized to B2M. (C) Expression of Ddr1 and Klf4 mRNA by qRTPCR in mammary tumors from C3(1)/TAg Klf4 ${ }^{+/+}$and C3(1)/TAg Klf4 ${ }^{+/-}$mice (bars, SEM). Reactions were normalized to RplpO. (D,E) ChIP analysis of a putative KLF4 binding site in MDA-MB-231 cells (bars, SEM). (F) Adhesion to low-attachment plates coated with indicated substrate (bars, SEM). 
A

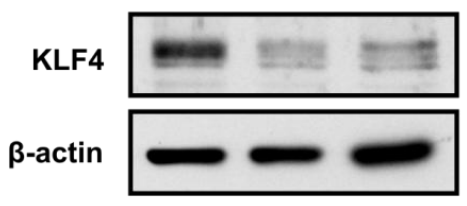

shRNA: Ctl KLF4(1) KLF4(2)

C

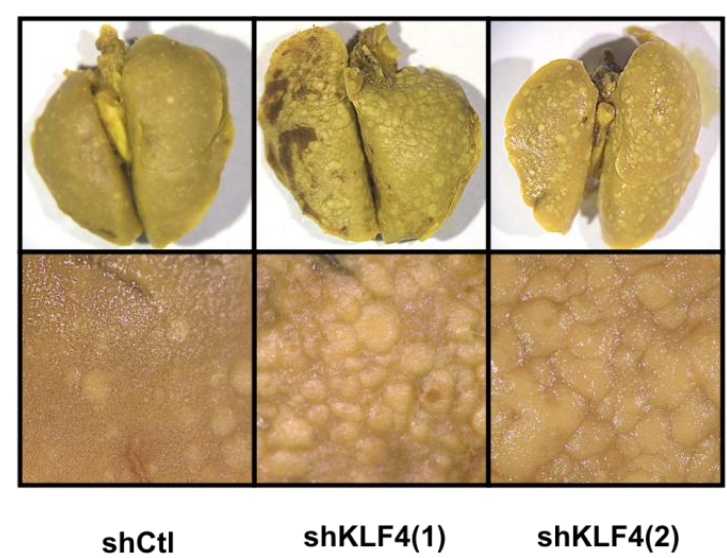

F

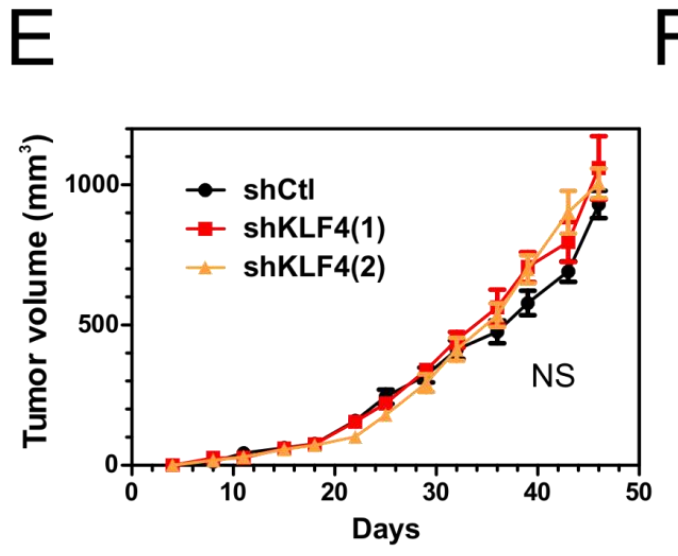

$\mathrm{B}$

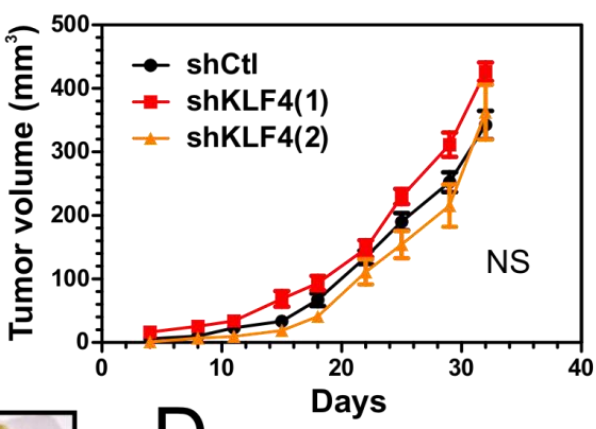

D

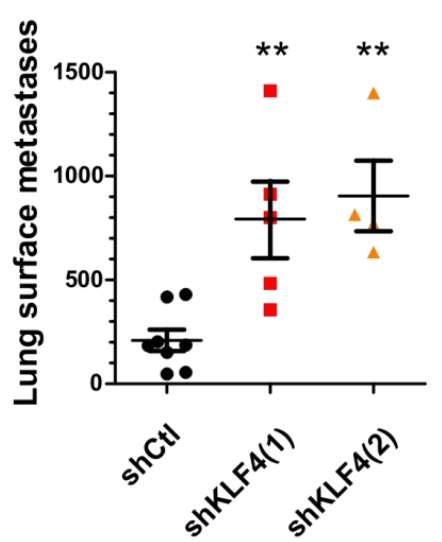

shCtl shKLF4(1) shKLF4(2)

$\mathrm{G}$

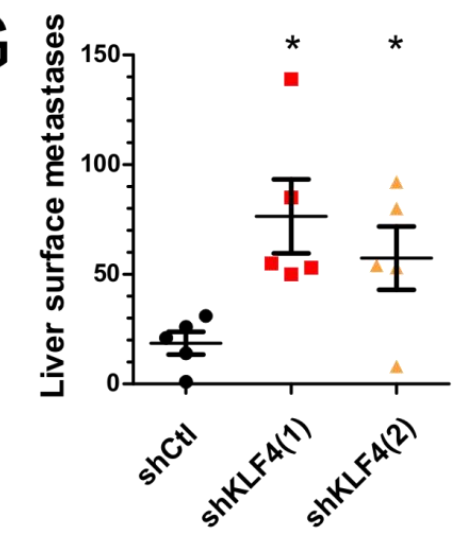

Figure 1. Suppression of KLF4 enhances spontaneous metastasis of MDA-MB-231 cells to the lungs and liver. 
A

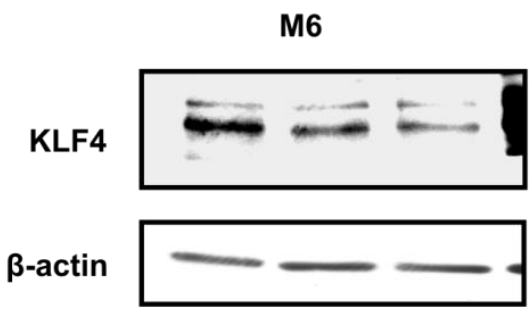

shRNA: Ctl KLF4(1) KLF4(2)
B

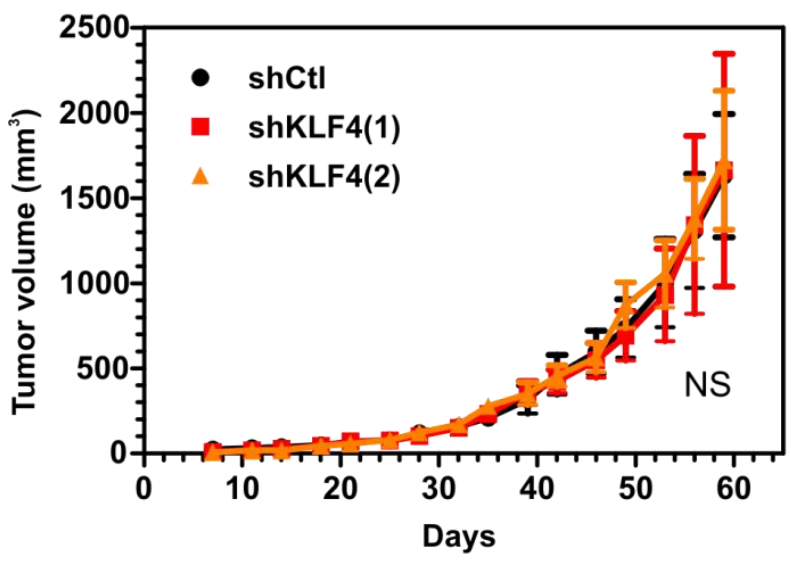

D

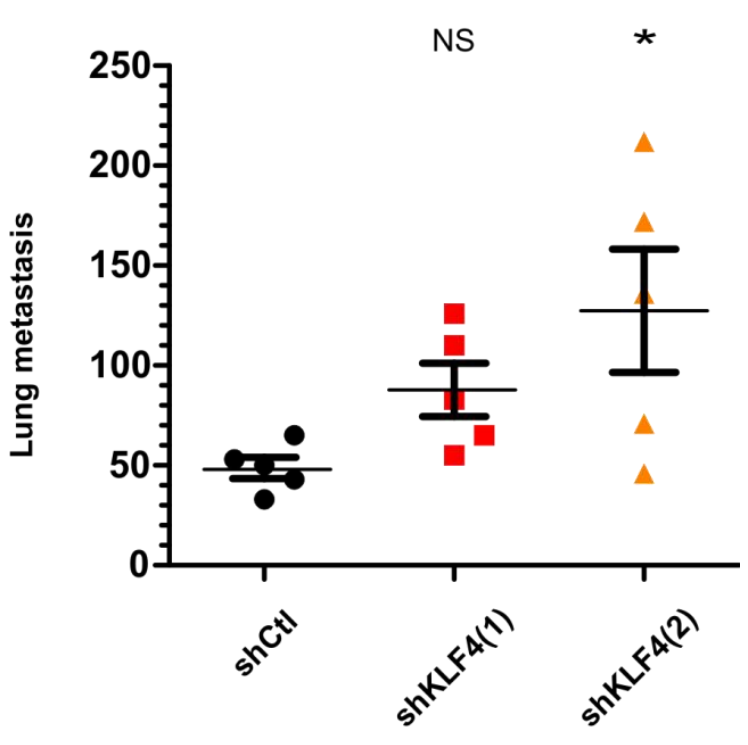

Figure 2. Suppression of KLF4 enhances spontaneous metastasis of M6 cells to the lungs. 
A

\section{B}
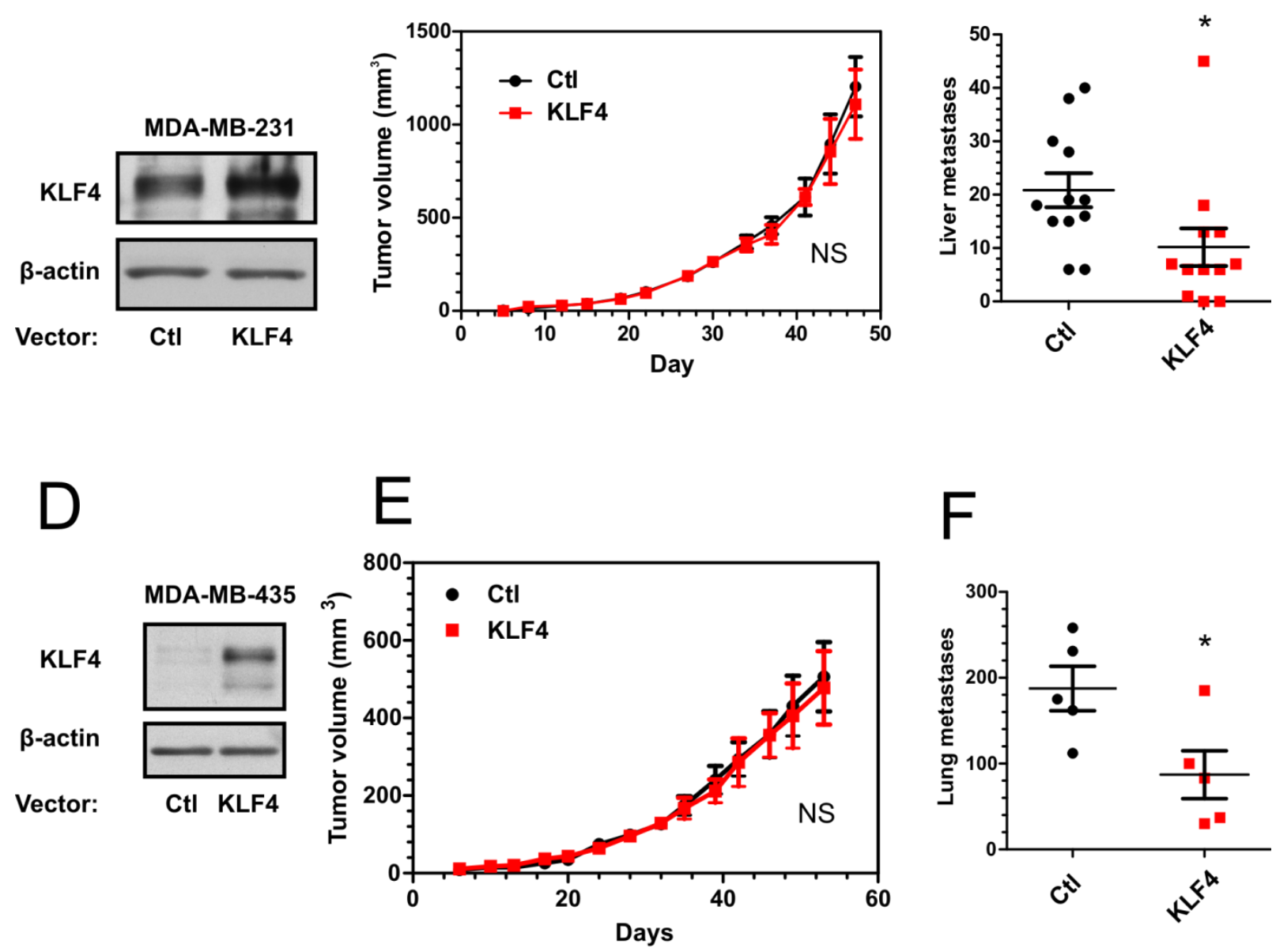

Figure 3. Overexpression of KLF4 suppresses spontaneous metastasis of MDA-MB-231 and MDA-MB-435 cells. 
A

B
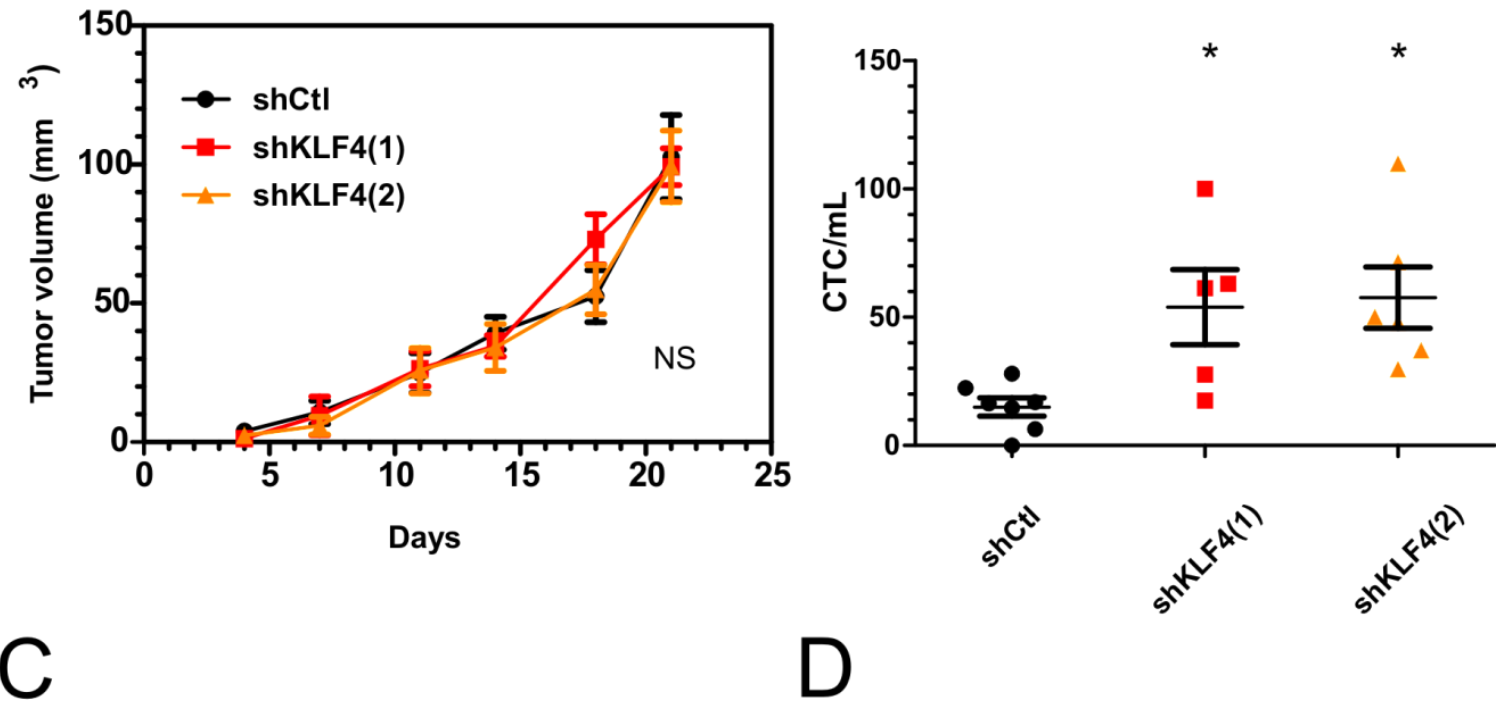

Transwell Migration

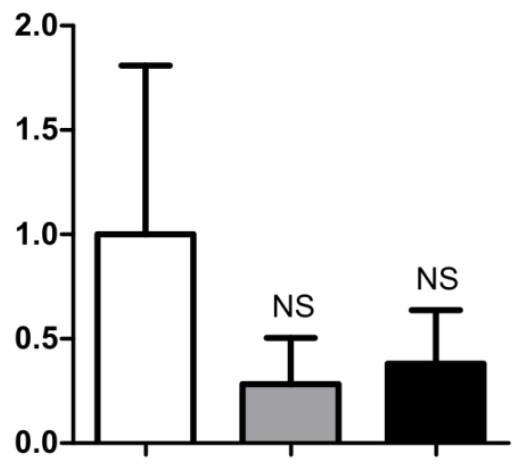

Transwell Invasion
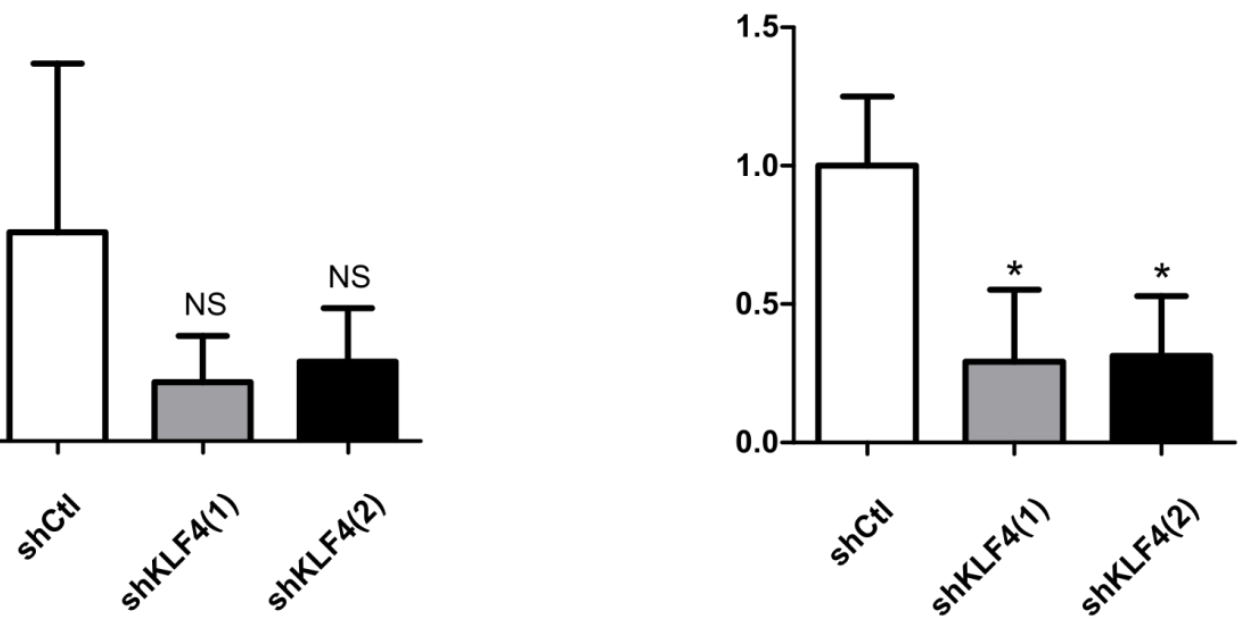

Figure 4. Effect of KLF4 expression on CTC levels, migration, and invasion of MDA-MB-231 cells. 

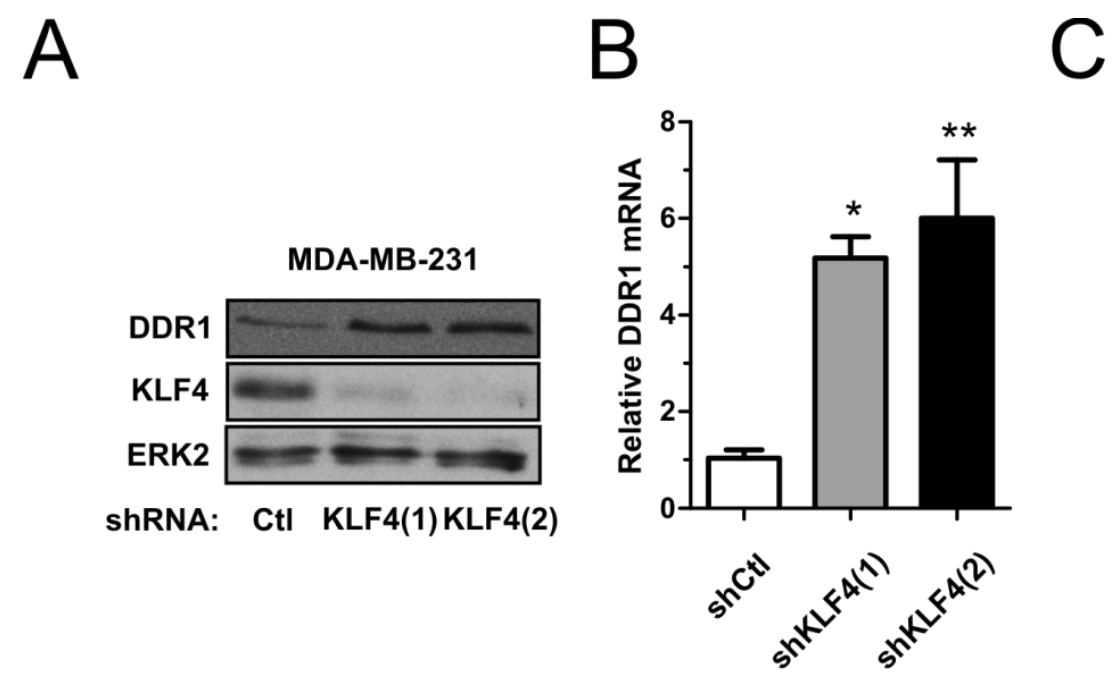

D

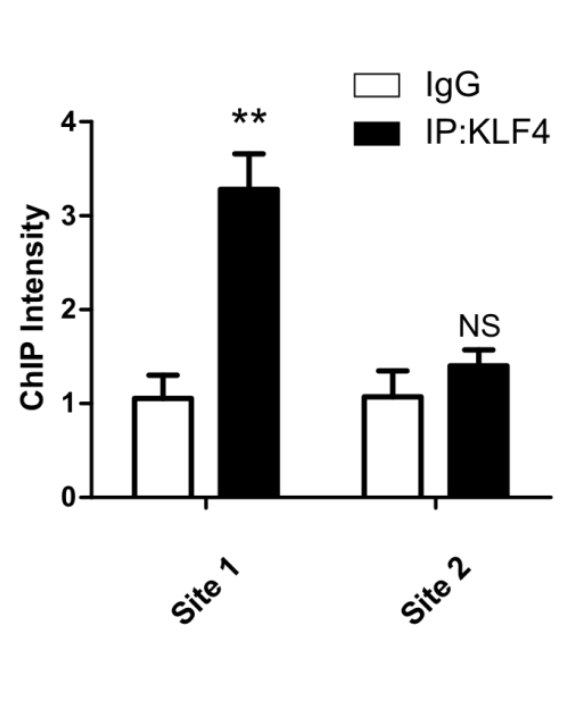

F

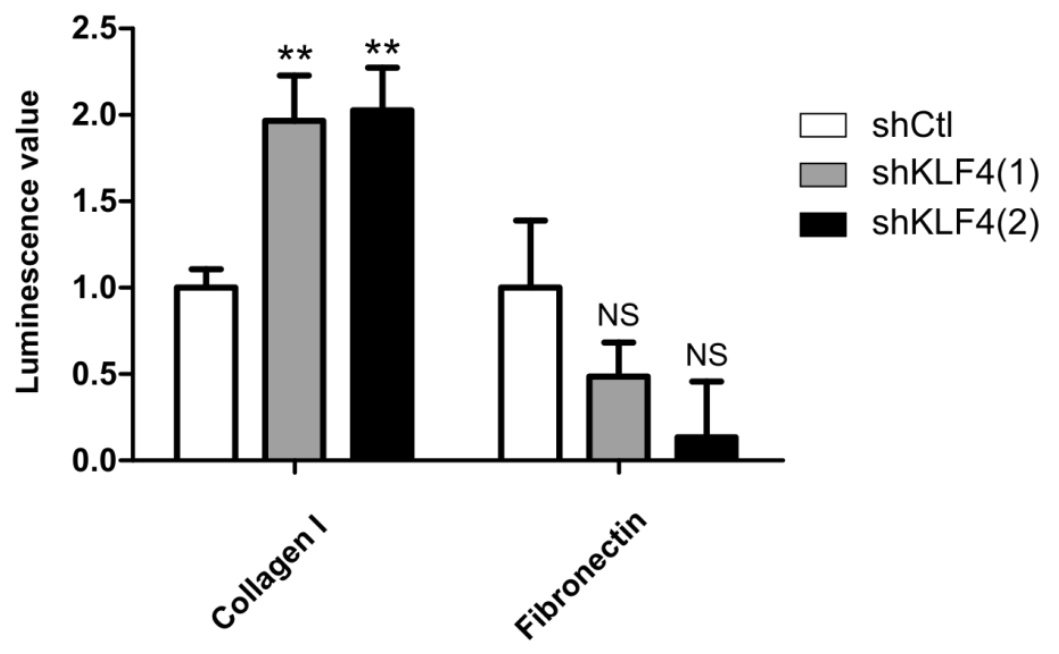

Figure 5. KLF4 suppresses DDR1 expression. 


\section{CHAPTER 4}

\section{Summary Discussion}

This dissertation addresses the function of the zinc finger transcription factors GLI1 and KLF4 in cancer. GLI1 is a potent transcriptional activator and effector protein of the Hedgehog signaling pathway, while KLF4 has similarly widespread functions in normal cells and in disease. GLI1 is regulated by degradation through the ubiquitin-proteasome system, and we provide evidence of a feedback mechanism determining GLI1 protein stability. While KLF4 can promote malignant properties in some contexts, we determined that KLF4 effectively suppresses metastasis of breast cancer cells.

\section{SOX9 inhibits $\beta$-TrCP-mediated degradation of GLI1}

The transcription factors SOX9 and GLI1 are implicated in the development of pancreatic ductal adenocarcinoma (PDA), and this dissertation describes a mechanistic function of SOX9 downstream of GLI1 in PDA cells. GLI1 directly promotes transcription of SOX9, and in a positive feedback relationship, SOX9 protein maintains the stability of GLI1. SOX9 interacted with the F-box domain of $\beta$-TrCP, disrupting the association of $\beta$-TrCP with SKP1 and inhibiting the function of the $\mathrm{SCF}^{\beta-\mathrm{TrCP}} \mathrm{E} 3$ ubiquitin ligase. Additionally, SOX9 promoted nuclear localization and destabilization of $\beta$-TrCP. In PDA cells, the loss of GLI1 protein and malignant properties in SOX9-deficient cells could be restored through suppression of $\beta$-TrCP. SOX9 was similarly important for cancer stem cell properties and the PDA cell side population. In addition

to GLI1, other $\mathrm{SCF}^{\beta-\mathrm{TrCP}}$ substrates such as $\beta$-catenin exhibit regulation by SOX9. This work 
demonstrates the role of GLI1-SOX9 feedback in stabilizing GLI1 protein, and shows that SOX9 can antagonize $\beta$-TrCP-mediated protein degradation.

\section{SOX9}

Our results implicate SOX9 expression as critical for GLI1 protein stability in pancreatic cancer cells. SOX9 is a transcription factor with important roles in developmental processes including sex determination and chondrogenesis, as well as cell differentiation in both embryonic and adult contexts (1-4). Expression of SOX9 contributes to development of numerous organs, involving all vertebrate germ layers (5).

The first of the SOX genes to be studied was sex-determining region Y (SRY), also known as testis determining factor (TDF) $(6,7)$. The SRY gene is found on the Y chromosome, and the gene product, commonly known as testis determining factor (TDF), is responsible for determining the male sex in almost all extant mammalian species. SRY contains a high mobility group (HMG) box domain. The HMG-box domain is found in some transcription factors, including SRY, where it functions to bind DNA. HMG-box domains are also found in other types of proteins, and the HMG-box domain may have functions such as protein-protein interaction (8). There are different types of HMG-box domain, and proteins containing an HMGbox domain with sufficient homology to SRY are called SRY box (SOX) proteins (9). A crucial function of SRY in determining the male sex is to promote expression of SOX9, which signals to induce Sertoli cell differentiation and the formation of testes (2). In conjunction with steroidogenic factor 1 (SF1) and other factors, SOX9 promotes expression of anti-Müllerian hormone (AMH) in Sertoli cells, blocking development of a female reproductive system (10). 
Mutations of SOX9 in humans are associated with campomelic dysplasia, characterized by skeletal abnormalities associated with the function of SOX9 in chondrocyte differentiation (11-13). Campomelic dysplasia is also associated with disorders of sexual development in affected males, which can result in normal female phenotypic development in an individual with the 46, XY karyotype. Consistent with the mechanisms of SOX9 in sexual determination, individuals with campomelic dysplasia and having a 46, XX karyotype are not affected by sex reversal (14).

\section{Hedgehog-SOX9 signaling}

In several contexts, SOX9 is already implicated as a downstream mediator of $\mathrm{Hh}$ signaling. Along with BMP signaling, Shh pathway activation helps establish SOX9 expression to regulate chondrocyte differentiation (15). In another study, Shh was overexpressed in mouse chondrocytes under control of the collagen II promoter/enhancer regulatory elements (16). This led to aberrant upregulation of SOX9, and skeletal abnormalities linked to defects in chondrocyte differentiation. Targeted inactivation of SOX9 in the skin prevents development of a hair stem cell niche and leads to alopecia (17). This study also demonstrated induction of SOX9 by Shh signaling, and suggested that the constitutive Hh pathway activation seen in basal cell carcinoma, a malignancy derived from hair follicular cells, leads to upregulation of SOX9. A genomics approach identifying conserved GLI binding sites identified the SOXE group, consisting of SOX8, SOX9, and SOX10, as regulated by Hh-Gli signaling, with a role in the development of Müller glial cells in the retina (18). Upregulation of SOX9 by Hh signaling has also been shown in basal cell carcinoma and pancreatic adenocarcinoma cells (19). 
Previous reports $(19,20)$, and data presented in our results, indicate GLI protein transcriptionally promotes SOX9 expression by interacting with an upstream response element. Our results therefore establish an additional means of positive feedback in Hh-GLI signaling, whereby SOX9 protein interacts with $\mathrm{SCF}^{\beta-\mathrm{TrCP}}$ to stabilize GLI1 protein. Another feedback mechanism is that GLI1 strongly promotes expression of itself at the transcriptional level. We observed this GLI1-SOX9 feedback in pancreatic cancer cells, where GLI may be activated by noncanonical mechanisms such as RAS, but both mechanisms seem likely to occur in diverse biological contexts of Hh pathway activation. Such examples would include normal and disease processes in which Hh-GLI is activated through Hh ligands, and malignancies such as basal cell carcinoma where the Hh pathway is activated through mutation of classical Hh pathway components.

\section{Hedgehog-SOX9 feedback in cancer}

It could be easily examined whether the GLI1-SOX9 feedback mechanism in pancreatic cancer cells extends to other contexts. To test this hypothesis, one would determine whether the suppression of SOX9, as can be accomplished through RNA interference, has a negative effect on GLI1 protein. As an initial screen, GLI transcriptional activity could be assessed through effects on GLII and PTCH mRNA, or through a GLI-binding site transcriptional reporter. The effect on GLI1 stability, and the dependence upon $\mathrm{SCF}^{\beta-\mathrm{TrCP}}$, could then be verified using the methods previously used in pancreatic cancer cells. We showed that the DNA binding domain of SOX9 is not required for GLI1 stability, and instead that the transactivation domain of SOX9 interacts with $\beta$-TrCP. By expressing a SOX9 construct lacking the DNA binding domain (HMG box), it should be possible to test whether a particular phenotype of SOX9 is dependent upon 
SOX9-mediated transcriptional regulation, or instead involves mechanisms such as stabilization of GLI protein.

Melanoma may represent the context most analogous to GLI signaling in pancreatic cancer. As in pancreatic cancer, GLI can be activated in melanoma by oncogenic RAS signaling (21). SOX9 is frequently expressed in human melanoma specimens $(22,23)$, and while overexpression of SOX9 can inhibit the growth of melanoma cells $(24,25)$, the contribution of endogenous SOX9 to malignant properties in this setting is unclear. We expect that GLI signaling in melanoma cells, partially mediated by RAS activation, will exhibit dependence on SOX9 expression, such that oncogenic GLI signaling may be blocked by SOX9 knockdown.

Oncogenic Hh-GLI signaling is activated in basal cell carcinoma by mutations in the Hh pathway, most often by inactivation of the tumor suppressor PTCH $(26,27)$, and occasionally through mutational activation of Smo (28). Interestingly, SOX9 is also highly expressed in basal cell carcinoma specimens and in a transgenic mouse basal cell carcinoma model $(17,29)$. SOX9 is also expressed in trichoepithelioma and trichilemmoma, tumors likewise thought to originate from follicles, as well as some other adnexal tumors, but not in Bowen's disease or in Merkel tumors. Notably, GLI1 overexpression in the skin leads not only to basal cell carcinoma, but also to development of trichoepithelioma, cylindroma, and trichoblastoma; all are follicular-derived tumors (30). The co-expression of SOX9 and GLI in normal follicles, and in follicle-related malignancies, has led to a hypothesis that SOX9 is a general effector of Hh signaling (29). By stabilizing GLI1, our data provide a means whereby this Hh-SOX9 signaling can promote tumorigenesis. A role for SOX9 in the regulation of hair follicles by Hh signaling (31) also 
seems quite plausible. SOX9 is not required for hair follicle formation, but is critical for a CD34 ${ }^{+}$ population of follicle stem cells (17).

\section{Hedgehog-SOX9 feedback in development}

Considering the vital developmental roles of both Hh signaling and SOX9, mutations of each pathway should result in some degree of phenotypic overlap if GLI-SOX9 feedback is relevant in normal physiological contexts of Hh signaling. This question is more complex, as the Hh pathway in mammalian development signals through the balance and interplay of GLI transcriptional activation $\left(\mathrm{GLI}^{\mathrm{A}}\right)$ and repression $\left(\mathrm{GLI}^{\mathrm{R}}\right)$ functions, distributed amongst the GLI family members (32). Moreover, GLI1 is seemingly not required for normal development (33). This result suggests the transactivation function of GLI2 can compensate for the loss of GLI1. In contrast, GLI2 loss results in embryonic lethality (34-36). Therefore, future studies into the involvement of SOX9 in Hh-GLI mediated developmental processes should take into account whether GLI2 is dependent upon SOX9.

The developmental defects associated with Hh mutations, including holoprosencephaly, appear at first glance to be distinct from SOX9-associated campomelic dysplasia. In fact, SOX9 has important roles in craniofacial development (37), and mutations of both SOX9 and of components of the Hh-GLI signal pathway have been associated with cleft lip and cleft palate (38). Whether craniofacial defects associated with SOX9 mutations result to some degree from destabilization of GLI protein is unclear. 


\section{Effect of SOX9 on $\beta$-TrCP substrates}

One of the more puzzling aspects of our results concerns the relative effects of SOX9 on GLI1 protein as compared to other substrates of $\mathrm{SCF}^{\beta-T r C P}$. Using both knockdown and overexpression of $\mathrm{SOX} 9$, we showed that $\mathrm{SOX} 9$ protein regulates $\mathrm{SCF}^{\beta-\operatorname{TrCP}}$ complex formation, specifically observing that the association of $\beta$-TrCP and SKP1 is inhibited by SOX9. This

mechanism suggests many substrates of $\mathrm{SCF}^{\beta-\operatorname{TrCP}}$ in addition to GLI1 should be stabilized by the presence of SOX9 (Chapter 2, Fig. 7A-C).

We found some evidence in support of the broader stabilization of $\beta$-TrCP substrates. MCL1 protein was noticeably decreased upon suppression of SOX9. This effect was proteasome-dependent, consistent with a stabilizing effect of SOX9. Additionally, $\beta$-catenin seemed to be stabilized by SOX9 as observed by immunoflourescence, and a TCF4 transcriptional reporter was markedly decreased following SOX9 knockdown. In contrast, the effect on $\beta$-catenin protein by immunoblot was less pronounced, and the levels of some $\beta$-TrCP substrates were unaffected by SOX9 knockdown (not shown). In fact, none of the other SCF ${ }^{\beta-\operatorname{TrCP}}$ substrates we examined were as dependent as GLI1 upon SOX9 expression. Others have reported that overexpression of GLI1 in cancer cells results in elevated nuclear expression of $\beta$ catenin (39). It would be interesting to examine whether this effect could be partly attributed to the stabilization of $\beta$-catenin by GLI-promoted SOX9.

A complicating factor in the analysis of the stability of $\mathrm{SCF}^{\beta-\operatorname{TrCP}}$ substrates, or indeed the substrates of any E3 ubiquitin ligase, is that proteins may be targeted by multiple E3 ligases. For example, GLI1 can also be ubiquitylated by Numb-Itch $(40,41)$, and $\beta$-catenin is also targeted for 
ubiquitylation by Jade-1 (42) and c-Cbl (43). At present, the overall effects of alternative E3 ligases upon SCF $\beta$-TrCP substrates are difficult to predict. There are hundreds of E3 ubiquitin ligases, including many without known substrates. Furthermore, it seems likely there are still unknown substrates of even well-characterized E3 ubiquitin ligases such as $\mathrm{SCF}^{\beta-\operatorname{TrCP}}$ and $\mathrm{SCF}^{\mathrm{CDC} 4}$.

\section{$\beta$-TrCP in cancer: Substrates and context-dependence}

While there are as yet no clinical therapies that specifically target the ubiquitin cascade, this approach could offer greater specificity in modulating the protein levels of specific E3 ubiquitin ligase substrates. Proteasome inhibitors, in contrast, are likely to affect all proteins with $26 \mathrm{~S}$ proteasome-dependent stability. By limiting stabilizing effects to a more narrow range of substrates, a therapy targeting the function of an E3 ligase could also offer decreased toxicities. Adverse events represent a serious drawback of current clinically approved proteasome inhibitors, and can include neurologic and hematologic effects (44-46). In contrast, therapeutic strategies to enhance the activity of the E3 ligase $\mathrm{SCF}^{\beta-\mathrm{TrCP}}$, as could be accomplished by antagonizing SOX9, could potentially have anticancer activity by destabilizing GLI1. An obstacle to the rational therapeutic inhibition of E3 ubiquitin ligases, is that the substrates and function in cancer of many E3 ligases is poorly understood.

This knowledge gap includes $\beta$-TrCP, as while $\mathrm{SCF}^{\beta-\operatorname{TrCP}}$ is seemingly one of the beststudied E3 ligases, the role of $\beta$-TrCP in human cancer is still unclear. In contrast, the Fbox/WD-repeat protein FBXW7/CDC4 is a well-documented tumor suppressor (47-50) with mutations in breast cancer $(51,52)$, ALL (53), gastric cancer (54), and other malignancies (55). 
As discussed in the Introduction (Chapter 1), overexpression of $\beta-\operatorname{TrCP}$ has been reported in some cancers (56-59), with evidence of increased tumors in $\beta$-TrCP-overexpressing GEM models (60). $\beta$-TrCP could promote malignancy through the degradation of substrates with tumor-suppressive properties, such as IкB proteins or PDCD4, and overexpression of $\beta$ - TrCP leads to unrestrained activation of NF-kB signaling (61).

There is also evidence that $\beta$ - $\operatorname{TrCP}$ might have tumor-suppressor functions. $\beta$ - $\operatorname{TrCP}$ could act as a tumor suppressor by promoting the degradation of oncoproteins such as GLI1 or $\beta$ catenin. $\beta$-TrCP mutations seem rare in cancer, but have been observed. The Okajima gastric cancer cell line harbors a $B T R C 2$ mutation leading to an $\mathrm{F} 462 \mathrm{~S}$ substitution of $\beta-\mathrm{TrCP} 2$ protein (62). This mutation is located within the WD-repeat region, and could therefore potentially alter or block substrate recognition. It would be informative to evaluate whether this mutation leads to the stabilization of GLI protein. Additionally, deletions of the $\beta$-TrCP WD region that could result in substrate stabilization are reported in prostate cancer (63).

The association of $\mathrm{SCF}^{\beta-\operatorname{TrCP}}$ with substrates classically involves substrate phosphorylation, followed by binding through a phosphodegron motif to $\beta$-TrCP, although proteomics methods also suggest propensity for non-classical binding (64). The stability of classical $\beta$-TrCP substrates is thus regulated by the activity of kinases, in addition to myriad other mechanisms. The interaction of $\beta-\operatorname{TrCP}$ with substrates is therefore likely to be highly context-dependent, and regulated by many factors aside from $\beta$ - TrCP protein expression. For example, our results indicate that SOX9 protein inhibits the interaction of GLI1 and $\beta$-TrCP. Methods to identify context-sensitive interaction between E3 ubiquitin ligases and their substrates will be of increasing relevance. A proteomics approach, using immunoprecipitation 
and mass spectrometry, could be used to identify and compare substrates of $\operatorname{SCF}^{\beta-\operatorname{TrCP}}(64,65)$ and other E3 ubiquitin ligases under varying experimental conditions. Previous efforts to screen for E3 ligase substrates have also used genetic methods such as global protein stability (GPS) profiling $(66,67)$. Such efforts could significantly improve understanding of the ubiquitinproteasome system in cancer.

\section{KLF4 suppresses metastasis of breast cancer cells}

In breast cancer, the transcription factor KLF4 promotes malignant properties and supports the cancer stem cell population, yet the overall role of KLF4 on tumor progression and metastasis in this context remains unclear. In this dissertation, we examine the effect of KLF4 expression on the spontaneous metastasis of breast cancer cells. We performed orthotopic injection of breast cancer cells in immunocompromised mice, finding that the stable suppression of KLF4 resulted in greater metastasis to the lungs and liver. Similarly, we found overexpression of KLF4 to inhibit metastasis. Suppression of KLF4 resulted in increased numbers of circulating tumor cells, yet the metastatic phenotype was unrelated to changes in primary tumor growth, and did not correlate with invasion and motility in 2D experiments. The expression of DDR1, a collagen receptor implicated in metastasis, was suppressed by KLF4, such that KLF4 knockdown led to increased DDR1 and adhesion to collagen. We propose that KLF4 suppresses metastasis through downregulation of DDR1. 


\section{KLF4 and DDR1 signaling}

We have shown that suppression of KLF4 leads to increased spontaneous metastasis of breast cancer cells in association with increased expression of DDR1. Significantly, KLF4 knockdown resulted in higher levels of CTCs, suggesting that KLF4 regulates the metastatic cascade at a stage before tumor cells arrive at distant sites. While endogenous DDR1 (68), as well as DDR2 $(69,70)$, were previously implicated as promoting metastasis, we have not yet examined the functional relationship of KLF4-DDR1 signaling in the metastasis of breast cancer cells. We hypothesize that silencing of DDR1 in KLF4 knockdown cells will lead to suppression of metastasis. Such a result would suggest that breast cancer metastasis is inhibited by the KLF4mediated suppression of DDR1.

If a KLF4-DDR1 signaling pathway is indeed relevant in the metastasis of breast cancer cells, the mechanisms downstream of DDR1 would be of interest. DDR1 is unusual amongst RTKs in that the activating ligand of DDR1 is collagen, a structural component of the extracellular matrix. It therefore seems that DDR1 could potentially affect metastasis through distinct processes. DDR1 autophosphorylation could stimulate pathways associated with DDR1 activation, including RAS, MAPK, and NF- $\kappa \mathrm{B}(71,72)$. Additionally, DDR1 expression might affect the interaction of cancer cells with the extracellular matrix, impacting processes such as matrix contraction and remodeling, invasion, motility, and intravasation.

It is unclear whether pathways downstream of DDR1 could suppress metastasis. The NF$\kappa \mathrm{B}$ pathway is known to protect cancer cells from apoptosis (73) and promote EMT (74). Additionally, NF- $\kappa \mathrm{B}$ signaling is highly linked to tumor initiation and progression (75-77), and 
may promote metastasis through CXCR4 (78). RAS and MAPK signaling likewise represent classic pathway associated with malignancy (79-82). While we have previously reported that KLF4 can promote malignant properties through RAS/MAPK signaling (83), this may not be true in every instance. JUNB and JUND, which may act as tumor suppressors (84), seem to be downregulated in metastasis with some consistency (85). In preliminary experiments we have observed decreased AP-1 reporter activity as a result of KLF4 suppression (not shown), although we have not directly examined the effect of KLF4 and DDR1 on FOS/JUN expression, and MAPKs are known to regulate AP-1 activity through several mechanisms (86).

Expression of DDR1 might affect metastasis through mechanisms independent from the direct effect DDR1 activation on cell signaling pathways. One such process is the interaction of cells with the ECM. Current, ongoing research in our lab suggest that suppression of KLF4 in MDA-MB -231 cells results in enhanced rigidity and contraction of a 3D collagen matrix, although it is not yet known whether this effect is a result of DDR1 upregulation. In addition to DDRs, the interaction of cells with collagen occurs through other receptors, including integrins (87-89). While several studies have indicated a causal relationship between breast cancer progression and stiffening of the ECM, the signal transduction from the collagen matrix was reported to occur through integrins receptors $(90,91)$. We plan to use a shRNA approach to address the specific contribution of DDR1 expression in breast cancer cells towards the rigidity and contraction of a 3D collagen matrix. It may also prove useful to evaluate the effects of KLF4 on the expression of alternate collagen receptors, including integrins such as $\alpha 1 \beta 1$. Our results indicate suppression of KLF4 leads to increased adhesion to collagen, and it will be of interest whether this effect is attributable to DDR1 upregulation. DDRs are known to regulate the behavior of cells in collagen matrices in vitro $(92,93)$, and evidence suggests the effects of 
DDR1 on cell spreading and migration over collagens are mediated through interaction with myosin IIA (94). It may then be of interest to examine whether KLF4 expression can regulate the co-immunoprecipitation of DDR1 and myosin IIA.

\section{KLF4 and the origins of metastasis}

Evidence from our lab and others indicate endogenous KLF4 can promote malignant properties of breast cancer cells in vitro and in vivo $(83,95-98)$. For this reason, we were surprised to discover KLF4 to be a highly effective metastasis suppressor of breast cancer cells in the NSG mouse model. One mechanism to explain these effects is that KLF4 expression could support the development of primary tumors, while subsequent downregulation of KLF4 facilitates metastasis. This could be assessed in the NSG model by comparing the expression of KLF4 in primary tumors and metastases. While a microarray study in MDA-MB-231 cells did not find KLF4 mRNA expression to be consistently altered in spontaneous metastasis (99), the PCR-based quantification of KLF4 in this context may prove more accurate.

While the severe immunodeficiency of the NSG mouse clearly enhances results in increased metastasis of breast cancer cell lines (99), this model system accordingly fails to account for the important roles of the immune system in the metastatic process (100). Still, the metastatic capability of cells in NSG mice has been shown to have clinical relevance. For example, the capability of primary melanoma cells to form spontaneous metastases in NSG mice corresponded with patient clinical outcome (101). 
Reports comparing gene expression in human patients of matched primary tumors and metastases are somewhat rare. In unsupervised hierarchical clustering of microarray-derived expression profiles, breast cancer metastases tend to cluster with the corresponding tumor of the same patient, as opposed to primary tumors or metastatic lesions of other patients (102). This was interpreted to signify that gene expression does not differ between primary tumors and metastasis. Similarly, it has been hypothesized that metastasis is largely preordained by the events in which normal cells are transformed into cancer cells $(103,104)$. Current perspectives on metastasis take into account that development of an invasive carcinoma occurs over a much longer time interval as compared to what is required for the subsequent development of metastasis $(105,106)$. The lack of well-defined genetic changes specific to metastasis must also be reconciled with compelling evidence that metastases occur from only a subset of the primary tumor cell population (107-109).

There is some evidence of differential gene regulation between primary tumors and metastasis. A meta-analysis of 28 total studies evaluated microarray data of primary tumors and distant metastasis (85). These included samples in which the authors concluded there was no difference between primary and metastatic gene expression (102). A metastatic signature of 79 genes was identified, and all except one were downregulated in metastasis. Examples of downregulated genes included some known metastasis suppressors as gelsolin (110) and connexin-43 (111). Additionally, a consistent set of differentially regulated genes has also been identified in lymph node metastasis of breast cancer (112). By restricting the metastatic specimens to lymph nodes, the variability imposed by the microenvironment in different sites such as lung and liver is reduced. 
While KLF4 could be downregulated in metastasis, there are other possibilities that could account for the seemingly discordant effects of KLF4 on different stages of carcinogenesis. Other changes in gene expression could compensate for the effects of KLF4. In accordance with the "seed and soil" hypothesis, it is also reasonable that KLF4 may exert distinct functions in the later steps of metastasis, in which breast cancer cells interact with a foreign microenvironment (113). It is still unexplained as to why KLF4 knockdown results in more CTCs in mice with comparably-sized primary tumors. Plausibly, suppression of KLF4 could lead to increased numbers of CTCs escaping the primary tumor into the vasculature, or the survival of tumor cells in circulation could be affected. Although suppression of KLF4 results in reduced motility and invasiveness of MBA-MD-231 cells in 2D culture, such effects of KLF4 in a 3D context are unknown. Given the effect of KLF4 on DDR1 expression and collagen adhesion, we plan to assess the effect of KLF4 on breast cancer cell invasion in a 3D collagen matrix, which might offer a better model of the in vivo phenotype.

\section{Circulating tumor cell detection and relationship to metastasis}

While the definition of a CTC is conceptually simple, the experimental determination of CTCs is complicated by their rarity in comparison to the normal cellular component of blood. Frequently, the presence of cytokeratins and the absence of the white blood cell (WBC) marker CD45 are considered indicative of CTCs (114). This immunologic approach is the basis of the only CTC assay that is currently approved for clinical use, having prognostic significance in several patient studies (115). In this method, any nonmalignant epithelial cells found in circulation would also be considered CTCs, although such CTCs are rare in patients without malignancy (116). 
Another concern with immunologic detection of CTCs is that cancer cells may undergo EMT, perhaps to promote invasion and survival in the bloodstream; CTCs with EMT characteristics could exhibit downregulation of epithelial markers used to define CTCs and thereby escape detection (117-119). Future advancements in the clinical detection of CTCs may benefit from analysis of EMT-related markers (115). Single-cell analyses of CTCs have revealed expression of EMT-related genes cancers of the breast (120) and prostate (121).

The detection of CTCs may also be accomplished using quantitative PCR methods. For example, evaluation of blood samples for expression of a transgene may indicate the presence of CTCs in a xenograft model (122). PCR-based CTC detection has also been applied with prognostic significance in a number of clinical studies (115). While we considered using PCR to detect CTCs in our experiments, the high GFP expression of MDA-MB-231 pGIPZ cell lines proved amenable to detection by flow cytometry. Whether this method of CTC detection can be used in other contexts will likely depend on the magnitude of fluorescence of the cells.

In M6 cells, lentiviral transduction of the same vector resulted in less expression of GFP as compared to MDA-MB-231 cells (not shown), and despite antibiotic selection, there remained a population of M6 cells without meaningful fluorescence above background. Although we attempted to evaluate CTCs from M6 primary tumors, the presence of cells lacking green fluorescence resulted in less reliable determination of CTCs, as these cells might be confused with mouse leukocytes. In contrast, by inoculating blood specimens from tumor-free mice with the MDA-MB-231 pGIPZ cell lines, we established to our satisfaction that we would not overlook an appreciable number of cells. It remains possible that some tumor cells could lose 
expression of GFP during in vivo proliferation, although most methods of CTC analysis rely on continued expression of some type of marker.

Overall, the gene expression of CTCs is somewhat labile in comparison to primary tumors and metastases. This effect may perhaps result from comparing the expression profile of single CTCs to that of bulk samples from solid tumor masses. In a study involving 62 patients with metastatic breast cancer, CTC profiling of a 35-gene set found discordant expression in $48 \%$ of patients as compared with the primary tumor (123). The CTC expression profile did not have prognostic significance. In breast cancer, CTCs are not necessarily predicted by the characteristics of primary tumors or axillary lymph node status (124).

One area of continuing uncertainty is the source of CTCs, as metastases could conceivably account for CTCs in circulation. A similar question is whether metastases can themselves give rise to other distant metastases (125), a proposition that has seen arguments on both sides $(126,127)$. Mathematical modeling of tumor growth and metastasis has predicted that metastases of metastases are not likely to comprise a significant portion of overall tumor burden in human patients (128). In our model system, we detected CTCs in mice lacking discernible lung metastases, suggesting that at this stage, the overall burden of cancer cells in the mice was primarily determined by the orthotopic tumor. 


\section{References}

1. de Crombrugghe, B., V. Lefebvre, and K. Nakashima. 2001. Regulatory mechanisms in the pathways of cartilage and bone formation. Curr Opin Cell Biol 13:721-727.

2. Kashimada, K. and P. Koopman. 2010. Sry: the master switch in mammalian sex determination. Development 137:3921-3930.

3. Barrionuevo, F. and G. Scherer. 2010. SOX E genes: SOX9 and SOX8 in mammalian testis development. Int J Biochem Cell Biol 42:433-436.

4. Sarkar, A. and K. Hochedlinger. 2013. The sox family of transcription factors: versatile regulators of stem and progenitor cell fate. Cell Stem Cell 12:15-30.

5. Jo, A., S. Denduluri, B. Zhang, Z. Wang, L. Yin, Z. Yan, R. Kang, L. L. Shi, J. Mok, M. J. Lee, and R. C. Haydon. 2014. The versatile functions of Sox9 in development, stem cells, and human diseases. Genes Dis 1:149-161.

6. Gubbay, J., J. Collignon, P. Koopman, B. Capel, A. Economou, A. Munsterberg, N. Vivian, P. Goodfellow, and R. Lovell-Badge. 1990. A gene mapping to the sexdetermining region of the mouse $\mathrm{Y}$ chromosome is a member of a novel family of embryonically expressed genes. Nature 346:245-250.

7. Sinclair, A. H., P. Berta, M. S. Palmer, J. R. Hawkins, B. L. Griffiths, M. J. Smith, J. W. Foster, A. M. Frischauf, R. Lovell-Badge, and P. N. Goodfellow. 1990. A gene from the human sex-determining region encodes a protein with homology to a conserved DNAbinding motif. Nature 346:240-244.

8. Stros, M., D. Launholt, and K. D. Grasser. 2007. The HMG-box: a versatile protein domain occurring in a wide variety of DNA-binding proteins. Cell Mol Life Sci 64:25902606.

9. Schepers, G. E., R. D. Teasdale, and P. Koopman. 2002. Twenty pairs of sox: extent, homology, and nomenclature of the mouse and human sox transcription factor gene families. Dev Cell 3:167-170.

10. De Santa, B. P., N. Bonneaud, B. Boizet, M. Desclozeaux, B. Moniot, P. Sudbeck, G. Scherer, F. Poulat, and P. Berta. 1998. Direct interaction of SRY-related protein SOX9 
and steroidogenic factor 1 regulates transcription of the human anti-Mullerian hormone gene. Mol Cell Biol 18:6653-6665.

11. Houston, C. S., J. M. Opitz, J. W. Spranger, R. I. Macpherson, M. H. Reed, E. F. Gilbert, J. Herrmann, and A. Schinzel. 1983. The campomelic syndrome: review, report of 17 cases, and follow-up on the currently 17-year-old boy first reported by Maroteaux et al in 1971. Am J Med Genet 15:3-28.

12. Foster, J. W., M. A. Dominguez-Steglich, S. Guioli, C. Kwok, P. A. Weller, M. Stevanovic, J. Weissenbach, S. Mansour, I. D. Young, P. N. Goodfellow, and . 1994. Campomelic dysplasia and autosomal sex reversal caused by mutations in an SRYrelated gene. Nature 372:525-530.

13. Wagner, T., J. Wirth, J. Meyer, B. Zabel, M. Held, J. Zimmer, J. Pasantes, F. D. Bricarelli, J. Keutel, E. Hustert, U. Wolf, N. Tommerup, W. Schempp, and G. Scherer. 1994. Autosomal sex reversal and campomelic dysplasia are caused by mutations in and around the SRY-related gene SOX9. Cell 79:1111-1120.

14. Mansour, S., C. M. Hall, M. E. Pembrey, and I. D. Young. 1995. A clinical and genetic study of campomelic dysplasia. J Med Genet 32:415-420.

15. Zeng, L., H. Kempf, L. C. Murtaugh, M. E. Sato, and A. B. Lassar. 2002. Shh establishes an Nkx3.2/Sox9 autoregulatory loop that is maintained by BMP signals to induce somitic chondrogenesis. Genes Dev 16:1990-2005.

16. Tavella, S., R. Biticchi, A. Schito, E. Minina, M. D. Di, A. Pagano, A. Vortkamp, W. A. Horton, R. Cancedda, and S. Garofalo. 2004. Targeted expression of SHH affects chondrocyte differentiation, growth plate organization, and Sox9 expression. J Bone Miner Res 19:1678-1688.

17. Vidal, V. P., M. C. Chaboissier, S. Lutzkendorf, G. Cotsarelis, P. Mill, C. C. Hui, N. Ortonne, J. P. Ortonne, and A. Schedl. 2005. Sox9 is essential for outer root sheath differentiation and the formation of the hair stem cell compartment. Curr Biol 15:13401351.

18. McNeill, B., C. Perez-Iratxeta, C. Mazerolle, M. Furimsky, Y. Mishina, M. A. ndradeNavarro, and V. A. Wallace. 2012. Comparative genomics identification of a novel set of temporally regulated hedgehog target genes in the retina. Mol Cell Neurosci 49:333-340. 
19. Eberl, M., S. Klingler, D. Mangelberger, A. Loipetzberger, H. Damhofer, K. Zoidl, H. Schnidar, H. Hache, H. C. Bauer, F. Solca, C. Hauser-Kronberger, A. N. Ermilov, M. E. Verhaegen, C. K. Bichakjian, A. A. Dlugosz, W. Nietfeld, M. Sibilia, H. Lehrach, C. Wierling, and F. Aberger. 2012. Hedgehog-EGFR cooperation response genes determine the oncogenic phenotype of basal cell carcinoma and tumour-initiating pancreatic cancer cells. EMBO Mol Med 4:218-233.

20. Bien-Willner, G. A., P. Stankiewicz, and J. R. Lupski. 2007. SOX9cre1, a cis-acting regulatory element located $1.1 \mathrm{Mb}$ upstream of SOX9, mediates its enhancement through the SHH pathway. Hum Mol Genet 16:1143-1156.

21. Stecca, B., C. Mas, V. Clement, M. Zbinden, R. Correa, V. Piguet, F. Beermann, and I. A. Ruiz. 2007. Melanomas require HEDGEHOG-GLI signaling regulated by interactions between GLI1 and the RAS-MEK/AKT pathways. Proc Natl Acad Sci U S A 104:58955900 .

22. Bakos, R. M., T. Maier, R. Besch, D. S. Mestel, T. Ruzicka, R. A. Sturm, and C. Berking. 2010. Nestin and SOX9 and SOX10 transcription factors are coexpressed in melanoma. Exp Dermatol 19:e89-e94.

23. Rao, P., G. N. Fuller, and V. G. Prieto. 2010. Expression of Sox-9 in metastatic melanoma--a potential diagnostic pitfall. Am J Dermatopathol. 32:262-266.

24. Passeron, T., J. C. Valencia, T. Namiki, W. D. Vieira, H. Passeron, Y. Miyamura, and V. J. Hearing. 2009. Upregulation of SOX9 inhibits the growth of human and mouse melanomas and restores their sensitivity to retinoic acid. J Clin Invest 119:954-963.

25. Shakhova, O., P. Cheng, P. J. Mishra, D. Zingg, S. M. Schaefer, J. Debbache, J. Hausel, C. Matter, T. Guo, S. Davis, P. Meltzer, D. Mihic-Probst, H. Moch, M. Wegner, G. Merlino, M. P. Levesque, R. Dummer, R. Santoro, P. Cinelli, and L. Sommer. 2015. Antagonistic cross-regulation between Sox 9 and Sox 10 controls an anti-tumorigenic program in melanoma. PLoS Genet 11:e1004877.

26. Hahn, H., C. Wicking, P. G. Zaphiropoulous, M. R. Gailani, S. Shanley, A. Chidambaram, I. Vorechovsky, E. Holmberg, A. B. Unden, S. Gillies, K. Negus, I. Smyth, C. Pressman, D. J. Leffell, B. Gerrard, A. M. Goldstein, M. Dean, R. Toftgard, G. Chenevix-Trench, B. Wainwright, and A. E. Bale. 1996. Mutations of the human homolog of Drosophila patched in the nevoid basal cell carcinoma syndrome. Cell 85:841-851. 
27. Gailani, M. R., M. Stahle-Backdahl, D. J. Leffell, M. Glynn, P. G. Zaphiropoulos, C. Pressman, A. B. Unden, M. Dean, D. E. Brash, A. E. Bale, and R. Toftgard. 1996. The role of the human homologue of Drosophila patched in sporadic basal cell carcinomas. Nat Genet 14:78-81.

28. Xie, J., M. Murone, S. M. Luoh, A. Ryan, Q. Gu, C. Zhang, J. M. Bonifas, C. W. Lam, M. Hynes, A. Goddard, A. Rosenthal, E. H. Epstein, Jr., and F. J. de Sauvage. 1998. Activating Smoothened mutations in sporadic basal-cell carcinoma. Nature 391:90-92.

29. Vidal, V. P., N. Ortonne, and A. Schedl. 2008. SOX9 expression is a general marker of basal cell carcinoma and adnexal-related neoplasms. J Cutan. Pathol 35:373-379.

30. Nilsson, M., A. B. Unden, D. Krause, U. Malmqwist, K. Raza, P. G. Zaphiropoulos, and R. Toftgard. 2000. Induction of basal cell carcinomas and trichoepitheliomas in mice overexpressing GLI-1. Proc Natl Acad Sci U S A 97:3438-3443.

31. Huntzicker, E. G. and A. E. Oro. 2008. Controlling hair follicle signaling pathways through polyubiquitination. J Invest Dermatol 128:1081-1087.

32. Altaba, A., C. Mas, and B. Stecca. 2007. The Gli code: an information nexus regulating cell fate, stemness and cancer. Trends Cell Biol 17:438-447.

33. Park, H. L., C. Bai, K. A. Platt, M. P. Matise, A. Beeghly, C. C. Hui, M. Nakashima, and A. L. Joyner. 2000. Mouse Gli1 mutants are viable but have defects in SHH signaling in combination with a Gli2 mutation. Development 127:1593-1605.

34. Mo, R., A. M. Freer, D. L. Zinyk, M. A. Crackower, J. Michaud, H. H. Heng, K. W. Chik, X. M. Shi, L. C. Tsui, S. H. Cheng, A. L. Joyner, and C. Hui. 1997. Specific and redundant functions of Gli2 and Gli3 zinc finger genes in skeletal patterning and development. Development 124:113-123.

35. Motoyama, J., J. Liu, R. Mo, Q. Ding, M. Post, and C. C. Hui. 1998. Essential function of Gli2 and Gli3 in the formation of lung, trachea and oesophagus. Nat Genet 20:54-57.

36. Matise, M. P., D. J. Epstein, H. L. Park, K. A. Platt, and A. L. Joyner. 1998. Gli2 is required for induction of floor plate and adjacent cells, but not most ventral neurons in the mouse central nervous system. Development 125:2759-2770. 
37. Lee, Y. H. and J. P. Saint-Jeannet. 2011. Sox9 function in craniofacial development and disease. Genesis. 49:200-208.

38. Dixon, M. J., M. L. Marazita, T. H. Beaty, and J. C. Murray. 2011. Cleft lip and palate: understanding genetic and environmental influences. Nat Rev Genet 12:167-178.

39. Liao, X., M. K. Siu, C. W. Au, Q. K. Chan, H. Y. Chan, E. S. Wong, P. P. Ip, H. Y. Ngan, and A. N. Cheung. 2009. Aberrant activation of hedgehog signaling pathway contributes to endometrial carcinogenesis through beta-catenin. Mod Pathol 22:839-847.

40. Di Marcotullio, L., E. Ferretti, A. Greco, S. E. De, A. Po, M. A. Sico, M. Alimandi, G. Giannini, M. Maroder, I. Screpanti, and A. Gulino. 2006. Numb is a suppressor of Hedgehog signalling and targets Gli1 for Itch-dependent ubiquitination. Nat Cell Biol 8:1415-1423.

41. Di Marcotullio, L., A. Greco, D. Mazza, G. Canettieri, L. Pietrosanti, P. Infante, S. Coni, M. Moretti, S. E. De, E. Ferretti, I. Screpanti, and A. Gulino. 2011. Numb activates the E3 ligase Itch to control Gli1 function through a novel degradation signal. Oncogene 30:65-76.

42. Chitalia, V. C., R. L. Foy, M. M. Bachschmid, L. Zeng, M. V. Panchenko, M. I. Zhou, A. Bharti, D. C. Seldin, S. H. Lecker, I. Dominguez, and H. T. Cohen. 2008. Jade-1 inhibits Wnt signalling by ubiquitylating beta-catenin and mediates Wnt pathway inhibition by pVHL. Nat Cell Biol 10:1208-1216.

43. Chitalia, V., S. Shivanna, J. Martorell, R. Meyer, E. Edelman, and N. Rahimi. 2013. c$\mathrm{Cbl}$, a ubiquitin E3 ligase that targets active beta-catenin: a novel layer of Wnt signaling regulation. J Biol Chem 288:23505-23517.

44. Richardson, P. G., P. Sonneveld, M. W. Schuster, D. Irwin, E. A. Stadtmauer, T. Facon, J. L. Harousseau, D. Ben-Yehuda, S. Lonial, H. Goldschmidt, D. Reece, J. F. SanMiguel, J. Blade, M. Boccadoro, J. Cavenagh, W. S. Dalton, A. L. Boral, D. L. Esseltine, J. B. Porter, D. Schenkein, K. C. Anderson, and Assessment of Proteasome Inhibition for Extending Remissions (APEX) Investigators. 2005. Bortezomib or high-dose dexamethasone for relapsed multiple myeloma. N Engl J Med 352:2487-2498.

45. Chen, D., M. Frezza, S. Schmitt, J. Kanwar, and Q. P. Dou. 2011. Bortezomib as the first proteasome inhibitor anticancer drug: current status and future perspectives. Curr Cancer Drug Targets 11:239-253. 
46. Robak, T., H. Huang, J. Jin, J. Zhu, T. Liu, O. Samoilova, H. Pylypenko, G. Verhoef, N. Siritanaratkul, E. Osmanov, J. Alexeeva, J. Pereira, J. Drach, J. Mayer, X. Hong, R. Okamoto, L. Pei, B. Rooney, d. van, V, F. Cavalli, and LYM-3002 Investigators. 2015. Bortezomib-based therapy for newly diagnosed mantle-cell lymphoma. N Engl J Med 372:944-953.

47. Lau, A. W., H. Fukushima, and W. Wei. 2012. The Fbw7 and betaTRCP E3 ubiquitin ligases and their roles in tumorigenesis. Front Biosci (Landmark Ed) 17:2197-2212.

48. Nakayama, K. I. and K. Nakayama. 2005. Regulation of the cell cycle by SCF-type ubiquitin ligases. Semin. Cell Dev Biol 16:323-333.

49. Welcker, M. and B. E. Clurman. 2008. FBW7 ubiquitin ligase: a tumour suppressor at the crossroads of cell division, growth and differentiation. Nat Rev Cancer 8:83-93.

50. Minella, A. C. and B. E. Clurman. 2005. Mechanisms of tumor suppression by the SCF(Fbw7). Cell Cycle 4:1356-1359.

51. Strohmaier, H., C. H. Spruck, P. Kaiser, K. A. Won, O. Sangfelt, and S. I. Reed. 2001. Human F-box protein hCdc4 targets cyclin $\mathrm{E}$ for proteolysis and is mutated in a breast cancer cell line. Nature 413:316-322.

52. Zhao, D., H. Q. Zheng, Z. Zhou, and C. Chen. 2010. The Fbw7 tumor suppressor targets KLF5 for ubiquitin-mediated degradation and suppresses breast cell proliferation. Cancer Res 70:4728-4738.

53. Malyukova, A., T. Dohda, L. N. von der, S. Akhoondi, M. Corcoran, M. Heyman, C. Spruck, D. Grander, U. Lendahl, and O. Sangfelt. 2007. The tumor suppressor gene hCDC4 is frequently mutated in human T-cell acute lymphoblastic leukemia with functional consequences for Notch signaling. Cancer Res 67:5611-5616.

54. Lee, J. W., Y. H. Soung, H. J. Kim, W. S. Park, S. W. Nam, S. H. Kim, J. Y. Lee, N. J. Yoo, and S. H. Lee. 2006. Mutational analysis of the hCDC4 gene in gastric carcinomas. Eur J Cancer 42:2369-2373.

55. Akhoondi, S., D. Sun, L. N. von der, S. Apostolidou, K. Klotz, A. Maljukova, D. Cepeda, H. Fiegl, D. Dafou, C. Marth, E. Mueller-Holzner, M. Corcoran, M. Dagnell, S. Z. Nejad, B. N. Nayer, M. R. Zali, J. Hansson, S. Egyhazi, F. Petersson, P. Sangfelt, H. Nordgren, D. Grander, S. I. Reed, M. Widschwendter, O. Sangfelt, and C. Spruck. 2007. 
FBXW7/hCDC4 is a general tumor suppressor in human cancer. Cancer Res 67:90069012.

56. Ougolkov, A., B. Zhang, K. Yamashita, V. Bilim, M. Mai, S. Y. Fuchs, and T. Minamoto. 2004. Associations among beta-TrCP, an E3 ubiquitin ligase receptor, betacatenin, and NF-kappaB in colorectal cancer. J Natl Cancer Inst 96:1161-1170.

57. Koch, A., A. Waha, W. Hartmann, A. Hrychyk, U. Schuller, A. Waha, K. A. Wharton, Jr., S. Y. Fuchs, S. D. von, and T. Pietsch. 2005. Elevated expression of Wnt antagonists is a common event in hepatoblastomas. Clin Cancer Res 11:4295-4304.

58. Dhawan, P. and A. Richmond. 2002. A novel NF-kappa B-inducing kinase-MAPK signaling pathway up-regulates NF-kappa B activity in melanoma cells. J Biol Chem 277:7920-7928.

59. Liu, J., K. G. Suresh Kumar, D. Yu, S. A. Molton, M. McMahon, M. Herlyn, A. ThomasTikhonenko, and S. Y. Fuchs. 2007. Oncogenic BRAF regulates beta-Trcp expression and NF-kappaB activity in human melanoma cells. Oncogene 26:1954-1958.

60. Kudo, Y., D. Guardavaccaro, P. G. Santamaria, R. Koyama-Nasu, E. Latres, R. Bronson, L. Yamasaki, and M. Pagano. 2004. Role of F-box protein betaTrcp1 in mammary gland development and tumorigenesis. Mol Cell Biol 24:8184-8194.

61. Muerkoster, S., A. Arlt, B. Sipos, M. Witt, M. Grossmann, G. Kloppel, H. Kalthoff, U. R. Folsch, and H. Schafer. 2005. Increased expression of the E3-ubiquitin ligase receptor subunit betaTRCP1 relates to constitutive nuclear factor-kappaB activation and chemoresistance in pancreatic carcinoma cells. Cancer Res 65:1316-1324.

62. Saitoh, T. and M. Katoh. 2001. Expression profiles of betaTRCP1 and betaTRCP2, and mutation analysis of betaTRCP2 in gastric cancer. Int J Oncol 18:959-964.

63. Gerstein, A. V., T. A. Almeida, G. Zhao, E. Chess, I. Shih, K. Buhler, K. Pienta, M. A. Rubin, R. Vessella, and N. Papadopoulos. 2002. APC/CTNNB1 (beta-catenin) pathway alterations in human prostate cancers. Genes Chromosomes Cancer 34:9-16.

64. Low, T. Y., M. Peng, R. Magliozzi, S. Mohammed, D. Guardavaccaro, and A. J. Heck. 2014. A systems-wide screen identifies substrates of the SCFbetaTrCP ubiquitin ligase. Sci Signal 7:rs8. 
65. Kim, T. Y., P. F. Siesser, K. L. Rossman, D. Goldfarb, K. Mackinnon, F. Yan, X. Yi, M. J. MacCoss, R. T. Moon, C. J. Der, and M. B. Major. 2015. Substrate trapping proteomics reveals targets of the betaTrCP2/FBXW11 ubiquitin ligase. Mol Cell Biol 35:167-181.

66. Yen, H. C. and S. J. Elledge. 2008. Identification of SCF ubiquitin ligase substrates by global protein stability profiling. Science 322:923-929.

67. Emanuele, M. J., A. E. Elia, Q. Xu, C. R. Thoma, L. Izhar, Y. Leng, A. Guo, Y. N. Chen, J. Rush, P. W. Hsu, H. C. Yen, and S. J. Elledge. 2011. Global identification of modular cullin-RING ligase substrates. Cell 147:459-474.

68. Valencia, K., C. Ormazabal, C. Zandueta, D. Luis-Ravelo, I. Anton, M. J. Pajares, J. Agorreta, L. M. Montuenga, S. Martinez-Canarias, B. Leitinger, and F. Lecanda. 2012. Inhibition of collagen receptor discoidin domain receptor-1 (DDR1) reduces cell survival, homing, and colonization in lung cancer bone metastasis. Clin Cancer Res 18:969-980.

69. Badiola, I., P. Villace, I. Basaldua, and E. Olaso. 2011. Downregulation of discoidin domain receptor 2 in A375 human melanoma cells reduces its experimental liver metastasis ability. Oncol Rep 26:971-978.

70. Ren, T., W. Zhang, X. Liu, H. Zhao, J. Zhang, J. Zhang, X. Li, Y. Zhang, X. Bu, M. Shi, L. Yao, and J. Su. 2014. Discoidin domain receptor 2 (DDR2) promotes breast cancer cell metastasis and the mechanism implicates epithelial-mesenchymal transition programme under hypoxia. J Pathol 234:526-537.

71. Ongusaha, P. P., J. I. Kim, L. Fang, T. W. Wong, G. D. Yancopoulos, S. A. Aaronson, and S. W. Lee. 2003. p53 induction and activation of DDR1 kinase counteract p53mediated apoptosis and influence p53 regulation through a positive feedback loop. EMBO J 22:1289-1301.

72. Das, S., P. P. Ongusaha, Y. S. Yang, J. M. Park, S. A. Aaronson, and S. W. Lee. 2006. Discoidin domain receptor 1 receptor tyrosine kinase induces cyclooxygenase-2 and promotes chemoresistance through nuclear factor-kappaB pathway activation. Cancer Res 66:8123-8130.

73. Dutta, J., Y. Fan, N. Gupta, G. Fan, and C. Gelinas. 2006. Current insights into the regulation of programmed cell death by NF-kappaB. Oncogene 25:6800-6816. 
74. Huber, M. A., N. Azoitei, B. Baumann, S. Grunert, A. Sommer, H. Pehamberger, N. Kraut, H. Beug, and T. Wirth. 2004. NF-kappaB is essential for epithelial-mesenchymal transition and metastasis in a model of breast cancer progression. J Clin Invest 114:569581.

75. Basseres, D. S. and A. S. Baldwin. 2006. Nuclear factor-kappaB and inhibitor of kappaB kinase pathways in oncogenic initiation and progression. Oncogene 25:6817-6830.

76. Karin, M. 2009. NF-kappaB as a critical link between inflammation and cancer. Cold Spring Harb. Perspect Biol 1:a000141.

77. Prasad, S., J. Ravindran, and B. B. Aggarwal. 2010. NF-kappaB and cancer: how intimate is this relationship. Mol Cell Biochem 336:25-37.

78. Helbig, G., K. W. Christopherson, P. Bhat-Nakshatri, S. Kumar, H. Kishimoto, K. D. Miller, H. E. Broxmeyer, and H. Nakshatri. 2003. NF-kappaB promotes breast cancer cell migration and metastasis by inducing the expression of the chemokine receptor CXCR4. J Biol Chem 278:21631-21638.

79. Bos, J. L. 1989. ras oncogenes in human cancer: a review. Cancer Res 49:4682-4689.

80. Pylayeva-Gupta, Y., E. Grabocka, and D. Bar-Sagi. 2011. RAS oncogenes: weaving a tumorigenic web. Nat Rev Cancer 11:761-774.

81. Dhillon, A. S., S. Hagan, O. Rath, and W. Kolch. 2007. MAP kinase signalling pathways in cancer. Oncogene 26:3279-3290.

82. Kim, E. K. and E. J. Choi. 2010. Pathological roles of MAPK signaling pathways in human diseases. Biochim Biophys Acta 1802:396-405.

83. Sharma, S. B., C. C. Lin, M. K. Farrugia, S. L. McLaughlin, E. J. Ellis, K. M. Brundage, M. A. Salkeni, and J. M. Ruppert. 2014. MicroRNAs 206 and 21 cooperate to promote RAS-extracellular signal-regulated kinase signaling by suppressing the translation of RASA1 and SPRED1. Mol Cell Biol 34:4143-4164.

84. Eferl, R. and E. F. Wagner. 2003. AP-1: a double-edged sword in tumorigenesis. Nat Rev Cancer 3:859-868. 
85. Daves, M. H., S. G. Hilsenbeck, C. C. Lau, and T. K. Man. 2011. Meta-analysis of multiple microarray datasets reveals a common gene signature of metastasis in solid tumors. BMC Med Genomics 4:56.

86. Karin, M. 1995. The regulation of AP-1 activity by mitogen-activated protein kinases. J Biol Chem 270:16483-16486.

87. Luo, B. H., C. V. Carman, and T. A. Springer. 2007. Structural basis of integrin regulation and signaling. Annu Rev Immunol 25:619-647.

88. Barczyk, M., S. Carracedo, and D. Gullberg. 2010. Integrins. Cell Tissue Res 339:269280.

89. Desgrosellier, J. S. and D. A. Cheresh. 2010. Integrins in cancer: biological implications and therapeutic opportunities. Nat Rev Cancer 10:9-22.

90. Levental, K. R., H. Yu, L. Kass, J. N. Lakins, M. Egeblad, J. T. Erler, S. F. Fong, K. Csiszar, A. Giaccia, W. Weninger, M. Yamauchi, D. L. Gasser, and V. M. Weaver. 2009. Matrix crosslinking forces tumor progression by enhancing integrin signaling. Cell 139:891-906.

91. Chaudhuri, O., S. T. Koshy, C. C. Branco da, J. W. Shin, C. S. Verbeke, K. H. Allison, and D. J. Mooney. 2014. Extracellular matrix stiffness and composition jointly regulate the induction of malignant phenotypes in mammary epithelium. Nat Mater 13:970-978.

92. Vogel, W. F., R. Abdulhussein, and C. E. Ford. 2006. Sensing extracellular matrix: an update on discoidin domain receptor function. Cell Signal 18:1108-1116.

93. Valiathan, R. R., M. Marco, B. Leitinger, C. G. Kleer, and R. Fridman. 2012. Discoidin domain receptor tyrosine kinases: new players in cancer progression. Cancer Metastasis Rev 31:295-321.

94. Huang, Y., P. Arora, C. A. McCulloch, and W. F. Vogel. 2009. The collagen receptor DDR1 regulates cell spreading and motility by associating with myosin IIA. J Cell Sci 122:1637-1646.

95. Yu, F., J. Li, H. Chen, J. Fu, S. Ray, S. Huang, H. Zheng, and W. Ai. 2011. Kruppel-like factor 4 (KLF4) is required for maintenance of breast cancer stem cells and for cell migration and invasion. Oncogene 30:2161-2172. 
96. Yu, F., Y. Shi, J. Wang, J. Li, D. Fan, and W. Ai. 2013. Deficiency of Kruppel-like factor KLF4 in mammary tumor cells inhibits tumor growth and pulmonary metastasis and is accompanied by compromised recruitment of myeloid-derived suppressor cells. Int J Cancer 133:2872-2883.

97. Okuda, H., F. Xing, P. R. Pandey, S. Sharma, M. Watabe, S. K. Pai, Y. Y. Mo, M. Iiizumi-Gairani, S. Hirota, Y. Liu, K. Wu, R. Pochampally, and K. Watabe. 2013. miR-7 suppresses brain metastasis of breast cancer stem-like cells by modulating KLF4. Cancer Res 73:1434-1444.

98. Farrugia, M. K., S. B. Sharma, C. C. Lin, S. L. McLaughlin, D. B. Vanderbilt, A. G. Ammer, M. A. Salkeni, P. Stoilov, Y. M. Agazie, C. J. Creighton, and J. M. Ruppert. 2015. Regulation of anti-apoptotic signaling by Kruppel-like factors 4 and 5 mediates lapatinib resistance in breast cancer. Cell Death Dis 6:e1699.

99. Iorns, E., K. Drews-Elger, T. M. Ward, S. Dean, J. Clarke, D. Berry, A. D. El, and M. Lippman. 2012. A new mouse model for the study of human breast cancer metastasis. PLoS One 7:e47995.

100. Vinay, D. S., E. P. Ryan, G. Pawelec, W. H. Talib, J. Stagg, E. Elkord, T. Lichtor, W. K. Decker, R. L. Whelan, H. M. Kumara, E. Signori, K. Honoki, A. G. Georgakilas, A. Amin, W. G. Helferich, C. S. Boosani, G. Guha, M. R. Ciriolo, S. Chen, S. I. Mohammed, A. S. Azmi, W. N. Keith, D. Bhakta, D. Halicka, H. Fujii, K. Aquilano, S. S. Ashraf, S. Nowsheen, X. Yang, B. K. Choi, and B. S. Kwon. 2015. Immune evasion in cancer: Mechanistic basis and therapeutic strategies. Semin. Cancer Biol.

101. Quintana, E., E. Piskounova, M. Shackleton, D. Weinberg, U. Eskiocak, D. R. Fullen, T. M. Johnson, and S. J. Morrison. 2012. Human melanoma metastasis in NSG mice correlates with clinical outcome in patients. Sci Transl Med 4:159ra149.

102. Weigelt, B., A. M. Glas, L. F. Wessels, A. T. Witteveen, J. L. Peterse, and L. J. van't Veer. 2003. Gene expression profiles of primary breast tumors maintained in distant metastases. Proc Natl Acad Sci U S A 100:15901-15905.

103. Bernards, R. and R. A. Weinberg. 2002. A progression puzzle. Nature 418:823.

104. Weinberg, R. A. 2007. Is metastasis predetermined? Mol Oncol 1:263-264.

105. Jones, S., W. D. Chen, G. Parmigiani, F. Diehl, N. Beerenwinkel, T. Antal, A. Traulsen, M. A. Nowak, C. Siegel, V. E. Velculescu, K. W. Kinzler, B. Vogelstein, J. Willis, and S. 
D. Markowitz. 2008. Comparative lesion sequencing provides insights into tumor evolution. Proc Natl Acad Sci U S A 105:4283-4288.

106. Vanharanta, S. and J. Massague. 2013. Origins of metastatic traits. Cancer Cell 24:410421.

107. Campbell, P. J., S. Yachida, L. J. Mudie, P. J. Stephens, E. D. Pleasance, L. A. Stebbings, L. A. Morsberger, C. Latimer, S. McLaren, M. L. Lin, D. J. McBride, I. Varela, S. A. Nik-Zainal, C. Leroy, M. Jia, A. Menzies, A. P. Butler, J. W. Teague, C. A. Griffin, J. Burton, H. Swerdlow, M. A. Quail, M. R. Stratton, C. Iacobuzio-Donahue, and P. A. Futreal. 2010. The patterns and dynamics of genomic instability in metastatic pancreatic cancer. Nature 467:1109-1113.

108. Yachida, S., S. Jones, I. Bozic, T. Antal, R. Leary, B. Fu, M. Kamiyama, R. H. Hruban, J. R. Eshleman, M. A. Nowak, V. E. Velculescu, K. W. Kinzler, B. Vogelstein, and C. A. Iacobuzio-Donahue. 2010. Distant metastasis occurs late during the genetic evolution of pancreatic cancer. Nature 467:1114-1117.

109. Gerlinger, M., A. J. Rowan, S. Horswell, J. Larkin, D. Endesfelder, E. Gronroos, P. Martinez, N. Matthews, A. Stewart, P. Tarpey, I. Varela, B. Phillimore, S. Begum, N. Q. McDonald, A. Butler, D. Jones, K. Raine, C. Latimer, C. R. Santos, M. Nohadani, A. C. Eklund, B. Spencer-Dene, G. Clark, L. Pickering, G. Stamp, M. Gore, Z. Szallasi, J. Downward, P. A. Futreal, and C. Swanton. 2012. Intratumor heterogeneity and branched evolution revealed by multiregion sequencing. N Engl J Med 366:883-892.

110. Fujita, H., F. Okada, J. Hamada, M. Hosokawa, T. Moriuchi, R. C. Koya, and N. Kuzumaki. 2001. Gelsolin functions as a metastasis suppressor in B16-BL6 mouse melanoma cells and requirement of the carboxyl-terminus for its effect. Int J Cancer 93:773-780.

111. Li, Z., Z. Zhou, D. R. Welch, and H. J. Donahue. 2008. Expressing connexin 43 in breast cancer cells reduces their metastasis to lungs. Clin Exp Metastasis 25:893-901.

112. Suzuki, M. and D. Tarin. 2007. Gene expression profiling of human lymph node metastases and matched primary breast carcinomas: clinical implications. Mol Oncol 1:172-180.

113. Fidler, I. J. 2003. The pathogenesis of cancer metastasis: the 'seed and soil' hypothesis revisited. Nat Rev Cancer 3:453-458. 
114. Marrinucci, D., K. Bethel, M. Luttgen, R. H. Bruce, J. Nieva, and P. Kuhn. 2009. Circulating tumor cells from well-differentiated lung adenocarcinoma retain cytomorphologic features of primary tumor type. Arch Pathol Lab Med 133:1468-1471.

115. Sun, Y. F., X. R. Yang, J. Zhou, S. J. Qiu, J. Fan, and Y. Xu. 2011. Circulating tumor cells: advances in detection methods, biological issues, and clinical relevance. J Cancer Res Clin Oncol 137:1151-1173.

116. Allard, W. J., J. Matera, M. C. Miller, M. Repollet, M. C. Connelly, C. Rao, A. G. Tibbe, J. W. Uhr, and L. W. Terstappen. 2004. Tumor cells circulate in the peripheral blood of all major carcinomas but not in healthy subjects or patients with nonmalignant diseases. Clin Cancer Res 10:6897-6904.

117. Lu, J., T. Fan, Q. Zhao, W. Zeng, E. Zaslavsky, J. J. Chen, M. A. Frohman, M. G. Golightly, S. Madajewicz, and W. T. Chen. 2010. Isolation of circulating epithelial and tumor progenitor cells with an invasive phenotype from breast cancer patients. Int J Cancer 126:669-683.

118. Hou, J. M., M. Krebs, T. Ward, R. Sloane, L. Priest, A. Hughes, G. Clack, M. Ranson, F. Blackhall, and C. Dive. 2011. Circulating tumor cells as a window on metastasis biology in lung cancer. Am J Pathol 178:989-996.

119. Gradilone, A., C. Raimondi, C. Nicolazzo, A. Petracca, O. Gandini, B. Vincenzi, G. Naso, A. M. Agliano, E. Cortesi, and P. Gazzaniga. 2011. Circulating tumour cells lacking cytokeratin in breast cancer: the importance of being mesenchymal. J Cell Mol Med 15:1066-1070.

120. Powell, A. A., A. H. Talasaz, H. Zhang, M. A. Coram, A. Reddy, G. Deng, M. L. Telli, R. H. Advani, R. W. Carlson, J. A. Mollick, S. Sheth, A. W. Kurian, J. M. Ford, F. E. Stockdale, S. R. Quake, R. F. Pease, M. N. Mindrinos, G. Bhanot, S. H. Dairkee, R. W. Davis, and S. S. Jeffrey. 2012. Single cell profiling of circulating tumor cells: transcriptional heterogeneity and diversity from breast cancer cell lines. PLoS One 7:e33788.

121. Chen, C. L., D. Mahalingam, P. Osmulski, R. R. Jadhav, C. M. Wang, R. J. Leach, T. C. Chang, S. D. Weitman, A. P. Kumar, L. Sun, M. E. Gaczynska, I. M. Thompson, and T. H. Huang. 2013. Single-cell analysis of circulating tumor cells identifies cumulative expression patterns of EMT-related genes in metastatic prostate cancer. Prostate 73:813826. 
122. Korpal, M., B. J. Ell, F. M. Buffa, T. Ibrahim, M. A. Blanco, T. Celia-Terrassa, L. Mercatali, Z. Khan, H. Goodarzi, Y. Hua, Y. Wei, G. Hu, B. A. Garcia, J. Ragoussis, D. Amadori, A. L. Harris, and Y. Kang. 2011. Direct targeting of Sec23a by miR-200s influences cancer cell secretome and promotes metastatic colonization. Nat Med 17:1101-1108.

123. Onstenk, W., A. M. Sieuwerts, M. Weekhout, B. Mostert, E. A. Reijm, C. H. van Deurzen, J. B. Bolt-de Vries, D. J. Peeters, P. Hamberg, C. Seynaeve, A. Jager, F. E. de Jongh, M. Smid, L. Y. Dirix, D. F. Kehrer, G. A. van, R. Ramirez-Moreno, J. Kraan, M. Van, J. W. Gratama, J. W. Martens, J. A. Foekens, and S. Sleijfer. 2015. Gene expression profiles of circulating tumor cells versus primary tumors in metastatic breast cancer. Cancer Lett 362:36-44.

124. Lucci, A., C. S. Hall, A. K. Lodhi, A. Bhattacharyya, A. E. Anderson, L. Xiao, I. Bedrosian, H. M. Kuerer, and S. Krishnamurthy. 2012. Circulating tumour cells in nonmetastatic breast cancer: a prospective study. Lancet Oncol 13:688-695.

125. Tait, C. R., D. Dodwell, and K. Horgan. 2004. Do metastases metastasize? J Pathol 203:515-518.

126. August, D. A., P. H. Sugarbaker, and P. D. Schneider. 1985. Lymphatic dissemination of hepatic metastases. Implications for the follow-up and treatment of patients with colorectal cancer. Cancer 55:1490-1494.

127. Sugarbaker, E. V., A. M. Cohen, and A. S. Ketcham. 1971. Do metastases metastasize? Ann Surg 174:161-166.

128. Bethge, A., U. Schumacher, A. Wree, and G. Wedemann. 2012. Are metastases from metastases clinical relevant? Computer modelling of cancer spread in a case of hepatocellular carcinoma. PLoS One 7:e35689. 


\section{Daniel B. Vanderbilt}

dvanderbilt@hsc.wvu.edu

\section{$\underline{\text { Education }}$}

West Virginia University School of Medicine, Morgantown, West Virginia

- Doctor of Philosophy, Cancer Cell Biology: May 2017 (expected). Dissertation: Regulation and Function of the Cancer Stem Cell Transcription Factors GLI and Krüppel-like Factor 4. Advisor: Dr. J. Michael Ruppert.

- Doctor of Medicine: May 2017 (expected).

Whitman College, Walla Walla, Washington

- Bachelor of Arts, Chemistry; with honors: May 2006; GPA 3.9/4.0. Advisor: Dr. Allison Calhoun.

\section{Research Experience}

West Virginia University School of Medicine, Morgantown, West Virginia

- Doctoral research, 2010-2015. Studied mechanisms governing the stability of GLI protein in pancreatic cancer cells, implicating a novel interaction between the transcription factor SOX9 and the E3 ubiquitin ligase SCF- $\beta$-TrCP, with consequences towards malignant properties and cancer stem cell traits. As a separate project, characterized the functional role of KLF4 in the spontaneous metastasis of breast cancer cells, involving suppression of the receptor tyrosine kinase DDR1. Advisor: Dr. J. Michael Ruppert, Department of Biochemistry and Mary Babb Randolph Cancer Center.

Washington State University, Pullman, Washington

- Honors thesis research, 2005-2006. Synthesis and characterization of cysteine-based compounds to coordinate rhenium and technetium for use as radiopharmaceuticals. Advisors: Dr. Paul Benny, Washington State University, Department of Chemistry; Dr. Allison Calhoun, Whitman College, Department of Chemistry.

\section{Honors/Awards}

\section{West Virginia University School of Medicine}

- Poster presentation award, $2^{\text {nd }}$ place- Van Liere Convocation and HSC Research Day, 2015.

\section{Whitman College}

- Walter Brattain Scholarship 2002-2006.

- Outstanding senior chemistry major 2006.

\section{Publications}


- Vanderbilt DB, Sharma SB, Lin CC, Ruppert JM. "KLF4 Suppresses Expression of the Receptor Tyrosine Kinase DDR1 in Breast Cancer Cells and Inhibits Spontaneous Metastasis." In submission.

- $\quad$ Deng W, Vanderbilt DB, Lin CC, Martin KH, Brundage KM, Ruppert JM. "SOX9 inhibits $\beta$-TrCP-mediated protein degradation to promote nuclear GLI1 expression and cancer stem cell properties." J Cell Sci. 2015, 128, 1123.

- Farrugia MK, Sharma SB, Lin CC, McLaughlin SL, Vanderbilt DB, Ammer AG, Salkeni MA, Stoilov P, Agazie YM, Creighton CJ, Ruppert JM. "Regulation of anti-apoptotic signaling by Kruppel-like factors 4 and 5 mediates lapatinib resistance in breast cancer." Cell Death Dis. 2015, 6, e1699.

\section{$\underline{\text { Leadership/Service }}$}

- Environmentors, 2013-2014. Mentoring of high school students to engage in scientific research.

- West Virginia University School of Medicine Strategic Planning, 2010-2011. Student representative for the five-year strategic planning committee, research section.

\section{$\underline{\text { Professional Affiliations }}$}

- American Association for the Advancement of Science (AAAS)

- American College of Physicians (ACP)

- National Eagle Scout Association (NESA) 